\title{
PLETHORA OF PLANTS - COLLECTIONS OF THE BOTANICAL GARDEN, FACULTY OF SCIENCE, UNIVERSITY OF ZAGREB (2): GLASSHOUSE SUCCULENTS
}

\section{Dubravka Sandev, Darko Mihelj \& Sanja Kovačić}

Botanical Garden, Department of Biology, Faculty of Science, University of Zagreb, Marulićev trg 9a, HR-10000 Zagreb, Croatia (e-mail: dubravka.sandev@biol.pmf.hr)

Sandev, D., Mihelj, D. \& Kovačić, S.: Plethora of plants - collections of the Botanical Garden, Faculty of Science, University of Zagreb (2): Glasshouse succulents. Nat. Croat. Vol. 27, No. 2, 407$420^{*}, 2018$, Zagreb.

In this paper, the plant lists of glasshouse succulents grown in the Botanical Garden from 1895 to 2017 are studied. Synonymy, nomenclature and origin of plant material were sorted. The lists of species grown in the last 122 years are constructed in such a way as to show that throughout that period at least 1423 taxa of succulent plants from 254 genera and 17 families inhabited the Garden's cold glasshouse collection.

Key words: Zagreb Botanical Garden, Faculty of Science, historic plant collections, succulent collection

Sandev, D., Mihelj, D. \& Kovačić, S.: Obilje bilja - zbirke Botaničkoga vrta Prirodoslovnomatematičkog fakulteta Sveučilišta u Zagrebu (2): Stakleničke mesnatice. Nat. Croat. Vol. 27, No. 2, 407-420*, 2018, Zagreb.

U ovom članku sastavljeni su popisi stakleničkih mesnatica uzgajanih u Botaničkom vrtu zagrebačkog Prirodoslovno-matematičkog fakulteta između 1895. i 2017. Uređena je sinonimka i nomenklatura te istraženo podrijetlo biljnog materijala. Rezultati pokazuju kako je tijekom 122 godine kroz zbirku mesnatica hladnog staklenika prošlo najmanje 1423 svojti iz 254 rodova i 17 porodica.

Ključne riječi: Botanički vrt PMF-a u Zagrebu, povijesne zbirke biljaka, zbirka mesnatica

\section{INTRODUCTION}

Following the comprehensive investigation of the plant collections in the Botanical Garden of the Faculty of Science, University of Zagreb (in further text "Botanical Garden" or "the Garden") initiated in 2012, we are continuing with the inventories of glasshouse succulent plants that went through our collections from the Botanical Garden's foundation until 2017. General facts about the Garden, its collections and glasshouses were published in our previous papers (cp. SAndev et al., 2013; Kovačić et al., 2014; Kovačıć 2015).

Following the principles established in Kovačıć (2015), we made an inventory of all existing and once-existing species of exotic succulent plants, including cacti, crassulas, aloes, agaves and other species within several families with similar physiologies, morp-

\footnotetext{
* Supplementary Material: Tab. 2 and 3 are available only in the electronic version of this article.

Dodatni materijal: Tabl. 2 i 3 dostupne su samo u elektroničkoj verziji ovoga članka.
} 
hologies and ecological needs (Fig. 1), grown together in the Garden's cold glasshouse collection since its foundation.

As succulent plants are very popular ornamentals all over the world, the interest in their cultivation and - nowadays - protection is vast. For example, it is noted that $68 \%$ of all threatened species of cacti (Cactaceae family) are recorded in botanic garden collections, while the most commonly recorded globally threatened cactus in botanic garden collections is Echinocactus grusonii Hildm. (Oldfield \& Hunt, 2010). In general, the natural distribution of succulent plants is global, but three continents are richer that the others: Africa, North and South America. Over the years, we grew in our collection more than 500 succulent species from the African Cape Floristic Region, and 30 from the Canaries and from Madagascar, respectively. From South America we had more than 300 species, mostly from Argentina. From North America we grew more than 250 species throughout the years, almost all of them from Mexico.

\section{The cold glasshouse collection}

History and living conditions of the Botanical Garden glasshouses were depicted in the paper Kovačić (2015). Non-native succulent species grown in the Botanical Garden collections overwinter or live permanently indoors. The Exhibition Glasshouse, built in 1891, has housed many species of succulent plants since its establishment; they were regularly exhibited in the open during the summer seasons (Fig. 2abcd) - a practice that is still kept up (Fig. 3ab). Within the Exhibition Glasshouse, the Cold Glasshouse (frigidarium) intended for the overwintering of succulents maintains a winter temperature of above $5{ }^{\circ} \mathrm{C}\left(\max .12{ }^{\circ} \mathrm{C}\right)$, so light heating is required. The amount of humidity is constantly kept very low, while regular ventilation is essential. Unfortunately, due to the complete reconstruction of the Exhibition Glasshouse, which started in 2015 and is not yet finished, the collection of succulents was temporarily rehoused in rather unsuitable premises, where it is much harder to obtain the needed winter conditions - consequently, some of the species withered over the last few years. As the glasshouses have been closed to the public for a century now, most of the indoor plants grown in larger pots are placed in the open during the summertime, so as to be accessible to our visitors. In future, when the old Exhibition Glasshouse is fully reconstructed, part of the succulent collection will gain a cold glasshouse-space of its own ("hladna kuća" - "cold house") and will be open to the general public.

During 2017 we thoroughly examined the published works, the Garden database and our own inventory records to establish the lists of exotic succulents living in the cold glasshouse collection between 1895 and 2017.

\section{MATERIAL \& METHOD}

Three main sources are used for constructing the lists of non-native succulents growing in the Botanical Garden's collections from its establishment until May 2017. As explained in Kovačıć (2015), three data-sources are available: (i) published records on the historic collection of the early days (1891-1896), (ii) a passive database of plants cultivated in the Garden since 1948 but not living today, and (iii) a active database of plants recently (2017) living in the cold glasshouse succulent collection, compiled from the attending gardener's glasshouse inventories.

The initial part of this study is based on a booklet written by the founder of the Botanical Garden, Professor Antun Heinz (HeInz, 1895-1896), which was constructed according 
to the classification of Plant Kingdom valid at that time, and was not precise in stating the details of the Garden's inventory. In most of the cases, only plant families and genera are named, as "containing a lot/many species", without any further details (origin, acquisition, living conditions, etc.). Accordingly, Tab. 1 lists the genera and families of glasshouse succulents grown in 1895, with very little explanation of the actual taxa. After the booklet of Heinz (1895-1896), there is a gap of more than 50 years throughout which the data on the glasshouse collections are missing.

Recent Garden records were founded in 1948 and ever since then we have been able to track our plant material with certainty. This database holds approx. 28000 cards divided in two parts: a passive database (plants not present in the Garden, but grown during the years) and an active database (plants recently grown in the Garden), which was digitalized in 2002 as a part of the Flora Croatica Database with restricted access (http://hirc.botanic.hr/ fcd/crofloradb/). The details on the collection's database and inventories after 1948 until today are explained in Kovačić (2015).

For most of the species already living in the glasshouses when this database was launched, the oldest cards state just "plant found growing in the Garden", without any further data on its origin, which was obviously unknown. One specimen of Cephalocereus senilis (Haw.) Pfeiff. and one of Mammillaria elongata DC. var. stella-aurata (Mart.) K. Schum. were the first succulent plants inventoried in the new database in 1947, followed by Crassula ericoides Haw., Gasteria brevifolia Haw., Harrisia bonplandii (Parm.) Britton \& Rose, H. pomanensis (F.A.C. Weber ex K. Schum.) Britton \& Rose, Rebutia minuscula K. Schum., Sedum hultenii Fröd., Senecio articulatus (L. f.) Sch. Bip. and Thelocactus setispinus (Engelm.) E.F. Anderson, all inventoried in 1949.

For most of the newcomers after 1948, the place of origin or the botanic garden of origin was noted, together with a year when the material was acquired: the oldest plant in the cold glasshouse succulent collection recorded in this manner was a Cylindrophyllum comptonii L. Bolus, obtained from Palermo (Italy) in 1950.

It is interesting to see that via Delectus (Index) Seminum publications the Garden obtained some native succulent seeds collected in the parts of the world with a high rate of endemism (South Africa, Central and Southern America) where collecting in the wild is nowadays strictly forbidden. Even today (Tab. 3) we hold some species listed in CITES (http://checklist. cites.org/\#/en), as well as invasive for example acc. to EASIN (http://alien.jrc.ec.europa.eu/ SpeciesMapper), which were freely distributed among botanic gardens before modern legislation was established.

Tab. 2 (with Tab. 3 available in the Supplement of the electronic version of this article) comprises the details extracted from the "passive" Garden-database, contained in the cards of individual taxa grown in the cold glasshouse conditions since 1948, but now missing in the recent collection (in Tabs. 2 and 3, "nn" stands for "unknown origin"). Again, as specified in Kovačıć (2015), examination of this part of the database inevitably led to the extraction of many synonyms, and double (even triple or more) entries for the same plant sample or species, as well as identification of several larger plant die-offs during the decades. Even during the last few years we have witnessed one of the succulent die-offs, since the Exhibition Glasshouse is under reconstruction and the collection was rehoused in other, less suitable, facilities.

The final part of this study is based on our active Garden database and recent records on the non-native succulents growing in the cold glasshouse collection. Tab. 3 depicts glasshouse succulents living in the Garden collections as of May, 2017. The basis of that list is the register of inventories, which is assembled biannually by the attending gardeners. While the active Garden database was thoroughly examined, many cards were replaced in the passive part of the records, while the plants perished in the meantime. 
Most of the plants in the recent cold glasshouse collection are represented by three to five clay-potted specimens, which are rejuvenated (vegetatively or germinatively), when and if possible. New plants for this collection are obtained through the Delectus Seminum network of inter-botanic-garden seed exchange, and grown in the Garden quarantine facilities. Occasionally, some cultivars are purchased from nurseries, garden centres or private collectors.

The general nomenclature of taxa follows the Plant List (http://www.theplantlist.org/) and Tropicos (http://www.tropicos.org/) databases. For African species the African Plant Database was consulted (http://www.ville-ge.ch/musinfo/bd/cjb/africa/). For specific cases (for example hybrids, cultivars) we used the specialized book-series "Illustrated Handbook of Succulent Plants" (Albers \& Meve, 2002; Eggli, 2001, 2002, 2003; Hartmann, 2001ab; IRISH \& IRISH, 2000).

\section{RESULTS \& DISCUSSION}

It is well known to any garden curator how difficult it is to compare plant data from various ages, due to the extensive synonymy and immense changes in the taxonomic and systematic relationships of plants, following ongoing research results over the years (further explained in Kovačıć, 2015). Therefore, for the simple "gardener-friendly" purpose we assembled the plants of interest here within "Dicotyledons" ("dicots", "Magnoliopsida") and "Monocotyledons" ("monocots", "Liliopsida"), and within them in families, genera and species. Here follow some explanations and clarifications of the data listed in Tabs. 1, 2 and 3, arranged by families of exotic succulents grown in the cold glasshouse collection of the Garden.

Tab. 1. Estimated collection of the cold glasshouse in 1895 (HeInz, 1895-1896).

\begin{tabular}{|c|c|c|c|c|}
\hline $\begin{array}{l}\text { Family } \\
\text { (acc. to Heinz' notes) }\end{array}$ & $\begin{array}{l}\text { Family } \\
\text { (acc. to Plant List } \\
\text { or Tropicos } \\
\text { databases) }\end{array}$ & $\begin{array}{l}\text { Scientific name } \\
\text { (acc. to Heinz' notes) }\end{array}$ & $\begin{array}{l}\text { Scientific name } \\
\text { (acc. to Plant List or } \\
\text { Tropicos databases) }\end{array}$ & Notes \\
\hline \multicolumn{5}{|l|}{ Dicotyledons } \\
\hline $\begin{array}{l}\text { Aizoaceae } \\
\text { (Mesembrianthemaceae) }\end{array}$ & Aizoaceae & Mesembrianthemum & Mesembryanthemum L. & $\begin{array}{l}\text { mostly from Cape } \\
\text { Province (south } \\
\text { Africa) }\end{array}$ \\
\hline $\begin{array}{l}\text { Aizoaceae } \\
\text { (Mesembrianthemaceae) }\end{array}$ & Aizoaceae & Tetragonia expansa & $\begin{array}{l}\text { Tetragonia tetragonioides } \\
\text { (Pall.) Kuntze }\end{array}$ & \\
\hline Asclepiadaceae & Apocynaceae & Stapelia & Stapelia L. & $\begin{array}{l}\text { different species from } \\
\text { Cape Province }\end{array}$ \\
\hline Cactaceae & Cactaceae & Cereus & Cereus Mill. & \\
\hline Cactaceae & Cactaceae & Echinocactus & Echinocactus Link \& Otto & \\
\hline Cactaceae & Cactaceae & Epiphyllum & Epiphyllum Haw. & \\
\hline Cactaceae & Cactaceae & Mammillaria & Mammillaria Haw. & \\
\hline Cactaceae & Cactaceae & Melocactus & Melocactus Link \& Otto & \\
\hline Cactaceae & Cactaceae & Opuntia & Opuntia Mill. & $\begin{array}{l}\text { lot of species, mostly } \\
\text { from temperate and } \\
\text { tropical America }\end{array}$ \\
\hline Cactaceae & Cactaceae & Opuntia Ficus indica & $\begin{array}{l}\text { Opuntia ficus-indica (L.) } \\
\text { Mill. }\end{array}$ & \\
\hline Cactaceae & Cactaceae & Opuntia missouriensis & Opuntia polyacantha Haw. & \\
\hline Cactaceae & Cactaceae & Peireskia & Pereskia Mill. & \\
\hline Cactaceae & Cactaceae & Phyllocactus & Epiphyllum Haw. & \\
\hline
\end{tabular}




\begin{tabular}{|c|c|c|c|c|}
\hline $\begin{array}{l}\text { Family } \\
\text { (acc. to Heinz' notes) }\end{array}$ & \begin{tabular}{l|} 
Family \\
(acc. to Plant List \\
or Tropicos \\
databases) \\
\end{tabular} & $\begin{array}{l}\text { Scientific name } \\
\text { (acc. to Heinz' notes) }\end{array}$ & $\begin{array}{l}\text { Scientific name } \\
\text { (acc. to Plant List or } \\
\text { Tropicos databases) }\end{array}$ & Notes \\
\hline Cactaceae & Cactaceae & Rhipsalis & Rhipsalis Gaertn. & \\
\hline Compositae & Asteraceae & Kleinia & Kleinia Mill. & \\
\hline Compositae & Asteraceae & Othona & Othonna L. & \\
\hline Compositae & Asteraceae & Senecio & Senecio L. & \\
\hline Crassulaceae & Crassulaceae & Bryophyllum calycinum & $\begin{array}{l}\text { Bryophyllum pinnatum } \\
\text { (Lam.) Oken }\end{array}$ & \\
\hline Crassulaceae & Crassulaceae & Cotyledon & Cotyledon L. & $\begin{array}{l}\text { lot of species, mostly } \\
\text { from Mexico and } \\
\text { Cape Province }\end{array}$ \\
\hline Crassulaceae & Crassulaceae & Crassula & Crassula L. & $\begin{array}{l}\text { lot of species, mostly } \\
\text { from Mexico and } \\
\text { Cape Province }\end{array}$ \\
\hline Crassulaceae & Crassulaceae & Echeveria & Echeveria DC. & $\begin{array}{l}\text { lot of species, mostly } \\
\text { from Mexico and } \\
\text { Cape Province }\end{array}$ \\
\hline Crassulaceae & Crassulaceae & Rochea & Rochea DC. & $\begin{array}{l}\text { lot of species, mostly } \\
\text { from Mexico and } \\
\text { Cape Province }\end{array}$ \\
\hline Crassulaceae & Crassulaceae & Sedum & Sedum L. & \\
\hline Crassulaceae & Crassulaceae & Semperivum & Sempervivum L. & \\
\hline Dioscoreaceae & Dioscoreaceae & $\begin{array}{l}\text { Testudinaria } \\
\text { elephantipes }\end{array}$ & $\begin{array}{l}\text { Dioscorea elephantipes } \\
\text { (L'Hér.) Engl. }\end{array}$ & $\begin{array}{l}\text { Heinz depicts as } \\
\text { "already withered" }\end{array}$ \\
\hline Euphorbiaceae & Euphorbiaceae & Euphorbia resinifera & Euphorbia resinifera O.Berg & \\
\hline Euphorbiaceae & Euphorbiaceae & Euphorbia & Euphorbia L. & different species \\
\hline Euphorbiaceae & Phyllanthaceae & Phyllanthus & Phyllanthus L. & \\
\hline Geraniaceae & Geraniaceae & Pelargonium & Pelargonium L’Hér. & from Cape Province \\
\hline \multicolumn{5}{|l|}{ Monocotyledons } \\
\hline Amaryllidaceae & Agavaceae & Agave americana & Agave americana $\mathrm{L}$. & \\
\hline Amaryllidaceae & Agavaceae & Agave & Agave L. & \\
\hline Amaryllidaceae & Asparagaceae & Beschorneria yuccoides & $\begin{array}{l}\text { Beschorneria yuccoides } \\
\text { K.Koch }\end{array}$ & \\
\hline Amaryllidaceae & Agavaceae & Fourcroya gigantea & Furcraea foetida (L.) Haw. & \\
\hline Bromeliaceae & Bromeliaceae & Dyckia & Dyckia Schult. \& Schult. f. & \\
\hline Liliaceae & Aloaceae & Aloe ferrox & Aloe ferox Mill. & \\
\hline Liliaceae & Aloaceae & Aloe & Aloe L. & $\begin{array}{l}\text { mostly from Cape } \\
\text { Province (south } \\
\text { Africa) }\end{array}$ \\
\hline Liliaceae & Aloaceae & Aloe succotrina & Aloe succotrina Lam. & \\
\hline Liliaceae & Aloaceae & Yucca & Yucca L. & \\
\hline
\end{tabular}

\section{“Dicotyledons" (“dicots", “Magnoliopsida")}

Most of the former and recent exotic plants of the Garden collections belong to the large and (also disputed) angiosperm group of "dicotyledons" or "magnoliopsida".

As seen in Tab. 1 (HeINz, 1895-1896), in the late $19^{\text {th }}$ century out of the total of 32 genera and 11 families, 26 "dicot"-genera from 8 families lived in the succulent glasshouse collection (Tab. 4). Compared to the other collections depicted in Heinz (1895-1896), where an astonishing amount of both indoor and outdoor plants contained in the collections soon after the Garden was formally established (1889) was described, Heinz's data on succulent plants are surprisingly humble. As described in Kovačić (2015), in his work of 1895-1896, 
Heinz listed up to (estimated) 250 plant taxa of the temperate glasshouse alone (possibly many more), approximately one hundred of them by name. Surprisingly, out of the cold glasshouse succulent collection Heinz listed only 6 "dicot" species by name, of which we still have in our collection only "Opuntia Ficus indica", while "Opuntia missouriensis" and Euphorbia resinifera existed in our collections also after 1947, but today are missing. We have never had "Bryophyllum calycinum", "Testudinaria elephantipes" and "Tetragonia expansa" after Heinz's times. Further 21 "dicot" genera are mentioned, but without much explanation ("lot of species...", "different species..." etc., as seen in Tab. 1). For example, the genera Melocactus, Rhipsalis, Kleinia, Othona, Cotyledon and Rochea are missing from the subsequent lists (Tab. 3). However, as emphasized in Kovačić (2015), this "lack" of representatives of certain genera - as well as sometimes of whole families - is highly relative, due to the taxonomic and nomenclatural variances: even today, different botanical sources classify plants in different ways. For example, the extremely diverse genus Phyllanthus of the current Phyllantaceae family contains approx. 60 genus synonyms alone, and was in Heinz's times classified within Euphorbiaceae. Since then, succulents of Phyllantaceae and Dioscoreaceae families have never been grown in the succulent collection.

According to our calculations (Tab. 2 \& 4), during the last 70 years, 1040 taxa passed through the cold glasshouse collection in addition to 151 genera of "dicot" succulent taxa, which are now missing, while we lack representatives of the Oxalidaceae family. Most abundant (Tab. 3) were the families of Cactaceae, Crassulaceae, Aizoaceae and Asclepiadaceae, as they are today. It should be emphasized that many scientific names are difficult to track, especially from the Cactaceae family (genera Cereus, Mammillaria, Parodia etc.) due to the immense synonymy. The Cactaceae family was in the past (Tab. 2) far more abundant than today (Tab. 3), with 83 genera and 537 taxa inventoried in the garden database since 1948. Many interesting species were ordered repeatedly from different botanic gardens during the years, among which an absolute "winner" is Mammillaria polythele Mart. subsp. polythele: it was ordered more than 40 times and under 8 different synonyms (Tab. 2); Astrophytum myriostigma Lem. was ordered 28 times under 7 different synonyms; Rebutia minuscula 23 times under 9 different synonyms since 1949, etc. Also, it is obvious that Delecti Semini of some botanical gardens were more popular than others: specifically, Berlin (300 orders), Barcelona (145), Wageningen (131), Gent (130) and Linz (107). However, for as much as 349 samples the source is unknown ("nn" in Tab. 2). Today, most of our recent succulents originate from Wageningen (19), Cluj-Napoca (14) and Monaco (13), while for as many as 39 species the source is unknown ("nn"). Some of those plants were in the 1950s and 1960s received from the Garden staff and visitors, and some were purchased, but without a known origin (acc. to Dr. Ljerka Regula - pers. cont.).

As of May 2017, we grew 220 "dicot"-succulent species from 82 genera and 11 families (Tab. 3 \& 4). The most abundant families are Cactaceae (66 taxa in 31 genera; Fig. 5ab) and Crassulaceae (55 taxa in 13 genera; Fig. 6abcdefg), followed by Aizoaceae (46 taxa in 24 genera; Fig. 7abc) and Euphorbiaceae (22 taxa of Euphorbia genus, Fig. 8). During the years, we lost 48 species from the family Asclepiadaceae, so today we grow 17 taxa in 5 genera Fig. 1, 9ab). Some families are represented by only a few succulent members, and are thus scarcely represented in our collection (for example Apocynaceae with 5 taxa during the years, Fig. 10).

In total (Tab. 3; Figs. 4, 6abcdef, 11), 50 \% of "dicot"-succulents grown today in our collection are placed in the CITES-lists (http://checklist.cites.org/\#/en). Furthermore, $13.64 \%$ of our recent "dicot"-succulents are listed as invasive in EASIN (http://alien. jrc.ec.europa.eu/SpeciesMapper; Fig. 5b). It is worth mentioning that some taxa are listed in both CITES- and EASIN-lists (Tab. 3; for example Euphorbia millii Desmoul., Opuntia ficus-indica (L.) Mill., O. maxima Mill). 
The oldest "dicot"-succulent grown in the Garden today is 64 years old Mammillaria compressa DC., followed by 60-year-old specimens of Ferocactus glaucescens (DC.) Britton \& Rose (Fig. 6d) and Graptopetalum paraguayense (N. E. Brown) E. Walther.

\section{"Monocotyledons" ("monocots", "Liliopsida")}

A smaller part of the succulent plants once grown in the cold glasshouse collection belongs to the angiosperm group of "monocotyledons" or "liliopsida".

At the end of the $19^{\text {th }}$ century, Heinz (1895-1896) depicted the representatives of at least 6 "monocot"-genera in 3 families at the time (Tab. 1 \& 4) held in the succulent collection: Amaryllidaceae (today Agavaceae), Liliaceae (today Aloaceae) and Bromeliaceae. Again, surprisingly, Heinz listed only 5 "monocot" species of his time by name, of which we still have in our collection Agave americana (cultivar 'Variegata'), "Aloe ferrox" (Fig. 4), and A. succotrina. "Fourcroya gigantea" existed in the succulent collection after 1947, but today is missing, while Beschorneria yuccoides have not been present since Heinz's time. The genera Dyckia and Yucca are mentioned, but without much explanation ("lot of species...", "different species..." etc., as seen in Tab. 1).

Tab. 4. Compilation of numbers of families, genera and taxa for Dicotyledons and Monocotyledons, extracted from three sources: 1895 - the booklet written by HeInz (1895-1896); 1948 - 2017 - the passive Garden database on the plants grown during that period, but recently non-existing in the succulent glasshouse collection; May, 2017 - the active Garden database on the succulents recently grown in the cold glasshouse collection. Asterisk $\left(^{*}\right)$ depicts the estimated number, due to the lack of precise data.

\begin{tabular}{|l|c|c|c|c|}
\hline \multirow{2}{*}{ Plant Group } & Year(s) & Families & Genera & Taxa \\
\hline \multirow{4}{*}{ Dicots } & 1895 & 8 & 26 & $150^{*}$ \\
\cline { 2 - 5 } & $1947-2016$ & 1 & 151 & $\mathbf{1 0 4 0}$ \\
\cline { 2 - 5 } & 2017 & 11 & 82 & 220 \\
\hline \multirow{4}{*}{ Monocots } & 1895 & 3 & 6 & $20^{*}$ \\
\cline { 2 - 5 } & $1947-2016$ & 0 & 5 & $\mathbf{1 0 4}$ \\
\cline { 2 - 5 } & 2017 & 5 & 13 & 55 \\
\hline \multirow{3}{*}{ In total } & 1895 & 11 & 32 & $170^{*}$ \\
\cline { 2 - 5 } & $1947-2016$ & 1 & 156 & $\mathbf{1 1 4 4}$ \\
\cline { 2 - 5 } & 2017 & 16 & 95 & 275 \\
\hline
\end{tabular}

According to our calculations (Tab. 2 \& 4), in the cold glasshouse succulent collection after 1947, 104 "monocot" taxa and 5 genera grew but are now missing. Out of that number, as many as 73 species belonged to the Aloaceae family, which is also the most numerous family within our "monocot"-succulents today (32 taxa in 4 genera; Figs. 4, $12 \mathrm{ab})$, followed by Agavaceae (18 taxa in 5 genera; Fig. 13ab).

The recent glasshouse succulent collection holds in total 55 "monocot" taxa from 13 genera and 5 families, out of which $45.5 \%$ are listed in CITES and $25.5 \%$ as invasive in EASIN (Tab. 3 \& 4; Figs. 4, 13a). Again, some taxa are placed in both lists (for example, Aloe vera (L.) Burm.f., A. ferox Mill. - Fig. 4), as seen in Tab. 3.

The oldest succulent plant grown in the Garden today is the 66-year-old "monocot" Gasteria bicolor Haworth var. bicolor, followed by the 64-year-old Gasteria acinacifolia (Jacquin) Haworth, the 59-year-old Haworthia attenuata (Haworth) Haworth var. radula (Jacquin) M.B. Bayer, etc. 
Finally, Tab. 4 depicts the relations in estimated numbers of families, genera and taxa of non-native succulents living in the cold glasshouse collection of the Botanical Garden (Faculty of Science, University of Zagreb) during the last 122 years.

\section{CONCLUSIONS}

According to a thorough examination of the available sources, we found that during the last 122 years, 1423 taxa of succulent plants (including cacti) from 254 genera and 17 families were cultivated in the cold glasshouse collection of the Botanical Garden, Faculty of Science, University of Zagreb.

HeInz (1895-1896) published 32 genera from 11 families inhabiting this collection in 1895: comparing with the data of other collections from the same source, we roughly estimated the number of succulent taxa of that time at 170 . After that, until the end of the WWII, there are no data on the glasshouse collections.

The passive part of the recent Garden-database, keeping the details on the plants that are today missing, shows that between 1948 and 20171144 taxa, 156 genera and 1 family non-existing today have passed through the cold glasshouse succulent collection.

The active part of the recent database holds the information on the plants cultivated in the Garden until May, 2017: accordingly, the cold glasshouse succulent plant collection contains 275 taxa from 95 genera and 16 families. Out of that number, $49.1 \%$ of taxa are listed in CITES and $16 \%$ in EASIN as invasive. In total, 91 species are new for the Garden collections and were not inventoried before 2017.

\section{ACKNOWLEDGEMENTS}

Source of photographs/Izvornik fotografija: 1, 4, 5ab, 6cdef, 8/1, 11, 12ab, 13 William Teocchi; 2abcd - Botanical Garden Archives; 3ab, 4b, 6ab, 7a, 9ab, 10, 13ab - Mirna Kirin; 6g, 7bc - Dubravka Sandev.

Received June 14, 2018

\section{REFERENCES}

Albers, F. \& Meve, U. (eds.), 2002: Illustrated Handbook of Succulent Plants: Asclepiadaceae. SpringerVerlag, Berlin, Heidelberg, New York, 1-318.

CITES (Checklist of CITES Species), 2015. Available from: http://checklist.cites.org/\#/en

EASIN (European Alien Species Information Network), 2012. Available from: http://alien.jrc.ec.europa. eu/SpeciesMapper

EgGLI, U. (ed.), 2001: Illustrated Handbook of Succulent Plants: Monocotyledons. Springer-Verlag, Berlin, Heidelberg, New York, 1-354.

EgGLI, U. (ed.), 2002: Illustrated Handbook of Succulent Plants: Dicotyledons. Springer-Verlag, Berlin, Heidelberg, New York, 1-488.

EgGLI, U. (ed.), 2003: Illustrated Handbook of Succulent Plants: Crassulaceae. Springer-Verlag, Berlin, Heidelberg, New York, 1-458.

Hartmann, H.E.K. (ed.) 2001a: Illustrated Handbook of Succulent Plants: Aizoaceae A-E. Springer-Verlag, Berlin, Heidelberg, New York, 1-285.

Hartmann, H.E.K. (ed.) 2001b: Illustrated Handbook of Succulent Plants: Aizoaceae F-Z. Springer-Verlag, Berlin, Heidelberg, New York, 1-371.

HeInZ, A., 1895-1896: Kr. Botanički vrt u Zagrebu. Glasnik Hrvatskoga naravoslovnoga društva, 8(1-6), $1-54$.

IrIsh, M. \& G., 2000: Agaves, yuccas, and related plants: a Gardener's guide. Timber Press Inc., 1-312. 
Kovačić, S., 2015: Plethora of plants - Collections of the Botanical Garden, Faculty of Science, University of Zagreb (1): Temperate glasshouse exotics - historic overview. Natura Croatica 24(2), 361-428 (397*).

Kovačić, S., Sandev, D., Mihelj, D. \& Stamenković, V., 2014: Win some, lose some - Statutorily strictly protected indigenous plant species in the Botanical Garden of the Faculty of Science, University of Zagreb (Croatia). Natura Croatica 23(2), 415-432.

Nikolić, T. (ed.), 2017: Flora Croatica Database (incl. the Botanical Garden Archives, with restricted access; SтAmenković, V., ed.: http://hirc.botanic.hr/fcd/crofloradb/). Faculty of Science, University of Zagreb. Last visit: December 2017.

Oldfield, S. \& Hunt D., 2010: The Conservation of Cacti and Succulents in Botanic Gardens. Journal of Botanic Gardens Conservation International 7(1), 15-17.

Sandev, D., Minelj D. \& Kovačıć, S., 2013: Meeting Target Eight - Ex situ conservation of Croatian threatened and statutorily protected plant species in the Botanical Garden of the Faculty of Science, University of Zagreb (Croatia). Natura Croatica 22(2), 343-362.

The International Plant Names Index, 2012: Published on the Internet http://www.ipni.org

The Plant List, Version 1.1., 2013: Published on the Internet http://www.theplantlist.org/

Tropicos.org., Missouri Botanical Garden, 2015: http://www.tropicos.org

\title{
SAŽETAK
}

\section{Obilje bilja - zbirke Botaničkoga vrta Prirodoslovno-matematičkog fakulteta Sveučilišta u Zagrebu (2): Stakleničke mesnatice}

\author{
D. Sandev, D. Mihelj i S. Kovačić
}

Prema propisima Europske unije koji se odnose na muzejsku djelatnost, potrebno je službeno registrirati i sve žive zbirke biljaka u botaničkim vrtovima, kako bi se moglo pratiti njihovo podrijetlo i put od sjemenke do odrasle biljke te spriječilo neovlašteno korištenje i moguća zlouporaba materijala.

Nakon analize zbirke toplog staklenika (KovAčıć, 2015) načinili smo analizu zbirki mesnatica (uključujući kaktuse) hladnog staklenika, koja je osim postojeće kartoteke obuhvatila i jedini sačuvani povijesni opis zbirki iz 19. stoljeća, objavljen u knjižici osnivača Botaničkoga vrta, profesora Antuna Heinza („Kr. Botanički vrt u Zagrebu“, 1895-1896). Iako vrlo šturo, iz knjižice doznajemo kako su do 1896. u staklenicima rasli pripadnici 32 roda mesnatica iz 11 porodica, broj kojih smo (usporedbom s podatcima istog izvora o drugim zbirkama iz toga vremena) procijenili na mogućih 170 svojti.

Kartoteka osnovana 1948. danas se sastoji od „pasivnog“ dijela, s odloženim karticama biljnih vrsta i nižih sistematskih kategorija (uključujući kultivare) koje su tijekom desetljeća uzgajane u Vrtu, a danas nedostaju; te „aktivnog“ dijela, s karticama biljnih vrsta i nižih sistematskih kategorija koje se trenutačno uzgajaju u Vrtu. Analizom rijetko korištenog, pasivnog dijela kartoteke utvrdili smo da je kroz zbirku mesnatica hladnog staklenika od 1948. do danas prošlo najmanje 1144 svojti koje nam danas nedostaju, te 156 rodova i jedna porodica.

S krajem svibnja 2017. u zbirci mesnatica hladnog staklenika obitavalo je 275 svojta iz 95 rodova i 16 porodica.

Usporedbom triju tablica s podatcima iz 1895/96. te iz današnje kartoteke, nalazimo kako je do danas kroz zbirku mesnatica hladnog staklenika prošlo barem 1423 biljnih svojti iz 254 rodova i 17 porodica. Međutim, potrebno je na kraju naglasiti da podatci o stakleničkim zbirkama između 1895. i 1948. godine ne postoje pa su ti brojevi zasigurno i veći. 


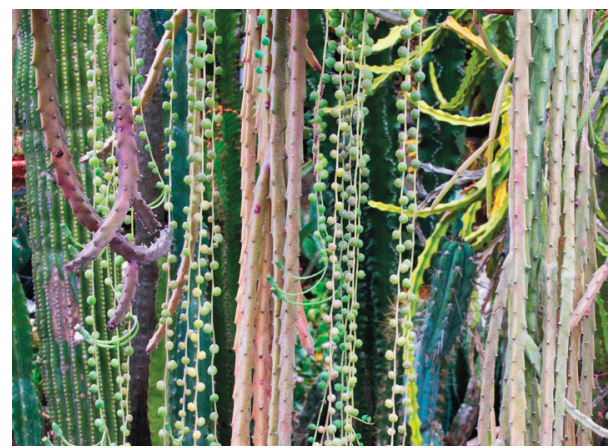

Fig. 1. Part of the recent "hanging" cold glasshouse succulent collection: Senecio rowleyanus $\mathrm{H}$. Jacobsen, Caralluma europaea (Guss.) N.E.Br., C. joannis Maire, Orbea variegata (L.) Haw.

S1. 1. Djelić današnjeg „,visećeg“ dijela zbirke stakleničkih mesnatica: Senecio rowleyanus H. Jacobsen, Caralluma europaea (Guss.) N.E.Br., C. joannis Maire, Orbea variegata (L.) Haw.

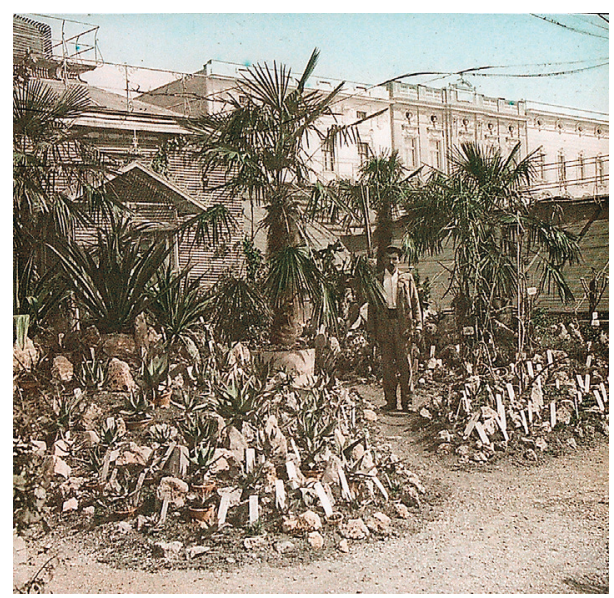

Fig. 2b. Part of the cold glasshouse succulent collection, displayed during summertime by the Exhibition Glasshouse (around 1910).

Sl. 2b. Dio zbirke stakleničkih mesnatica ljeti izložen uz izložbeni staklenik (oko 1910.).

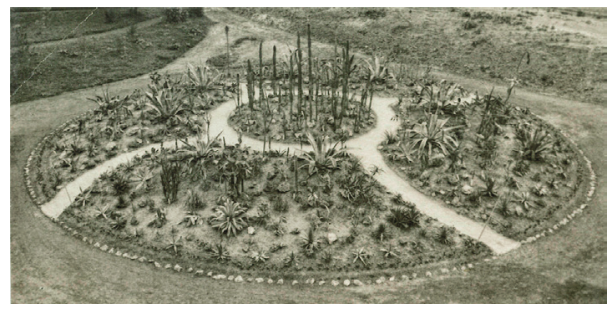

Fig. 2a. ,The Big Wheel“: part of the cold glasshouse succulent collection, displayed during summertime in front of the Gardener's House (nowadays Garden management; probably late 1890s).

Sl. 2a. Veliki kotač, krug ili kolo: dio zbirke stakleničkih mesnatica ljeti izložen ispred Vrtlarske kuće (danas uprava Vrta; vjerojatno krajem 1890ih).

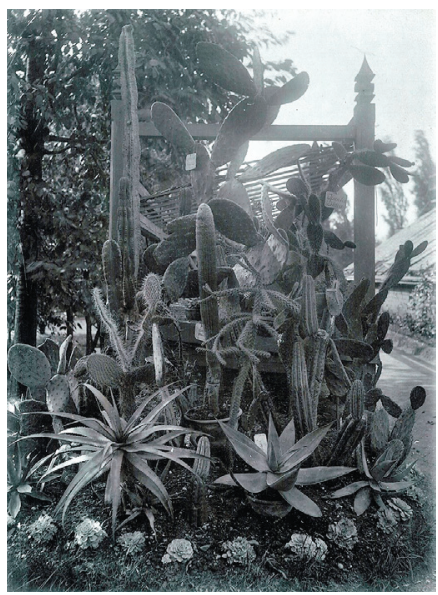

Fig. 2c. Garden bed with ornamental eaves, holding a part of the cold glasshouse succulent collection, displayed during summertime by the Exhibition Glasshouse (probably between 1920 and 1930).

S1. 2c. Gredica s ukrasnom strehom i zbirkom stakleničkih mesnatica ljeti izloženom uz izložbeni staklenik (vjerojatno između 1920. i 1930.). 


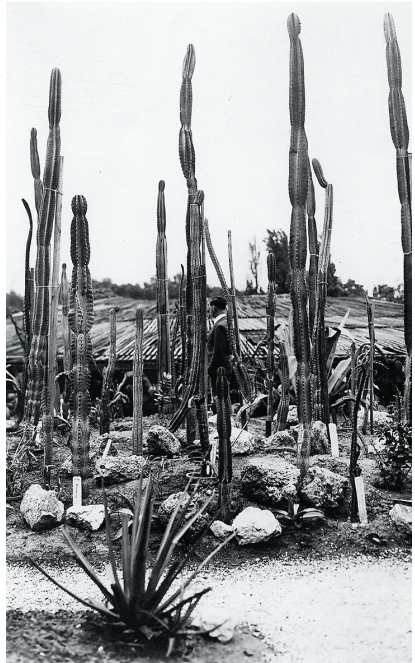

Fig. 2d. Part of the cold glasshouse succulent collection, displayed during summertime in front of the Gardener's House (nowadays Garden management; probably 1930-ies).

S1. 2d. Zbirka stakleničkih mesnatica ljeti izložena ispred Vrtlarske kuće (danas uprava Vrta; 1930-ih).

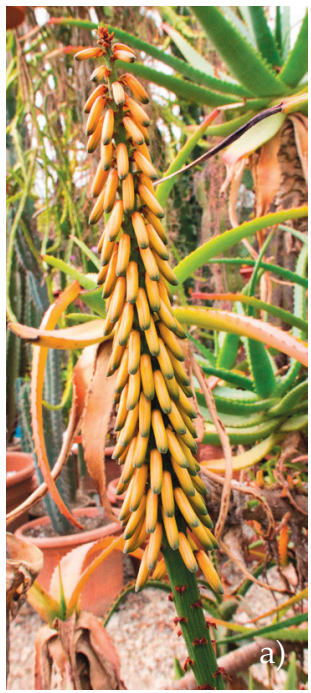

Fig. 4ab. The popular ornamental plant, bitter aloe (Aloe ferox Mill.), has lived in the Garden cold glasshouse collection since its foundation; it is placed in both CITES and EASIN lists.

Sl. 4ab. Popularna uresna mesnatica gorki aloj (Aloe ferox Mill.) nalazi se u našoj zbirci od samoga osnutka, a danas je na CITES i EASIN-listama.
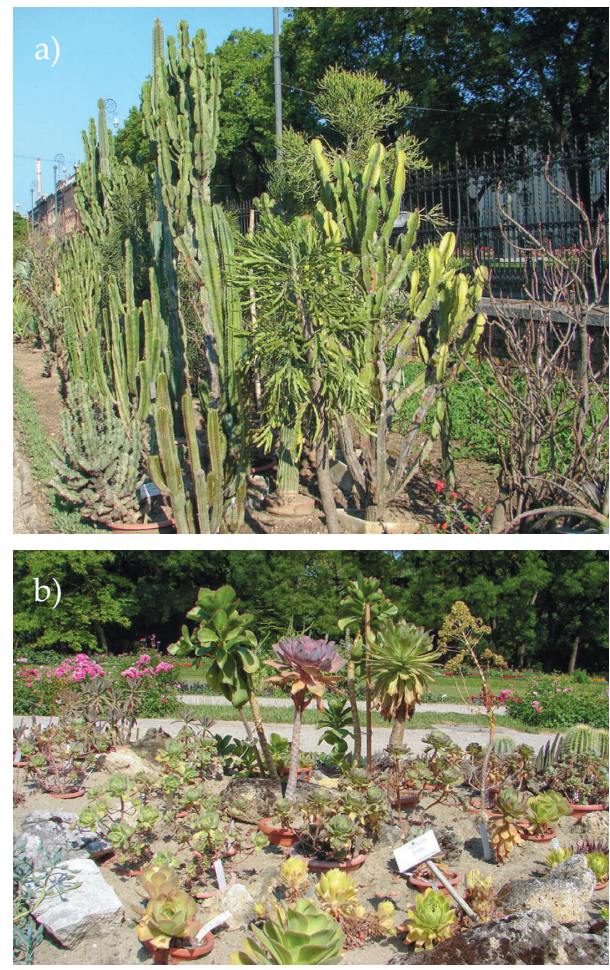

Fig. 3. Due to the lack of permanent display premises, large portions of the cold glasshouse succulent collection are still placed in the open during summertime: a) Large plants with pots are placed along the northern part of the wrought-iron fence (Marko Marulić Square)... b) ... while the smaller individuals are placed by the flower parterre in the middle part of the Garden.

S1. 3. Zbog nedostatka prostora za trajni smještaj biljaka, dostupnog posjetiteljima, zbirka stakleničkih mesnatica i danas se ljeti iznosi na otvoreno: a) Velike biljke u posudama ukapaju se uz sjevernu ogradu Vrta, duž Marulićevog trga... b) ...dok se manje biljke smještaju uz cvjetni parter u središnjem dijelu Vrta.

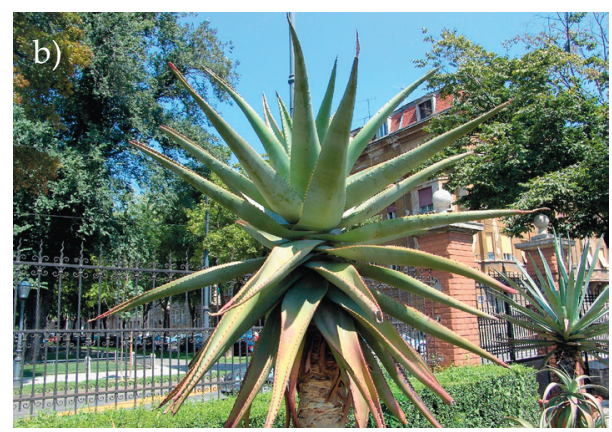




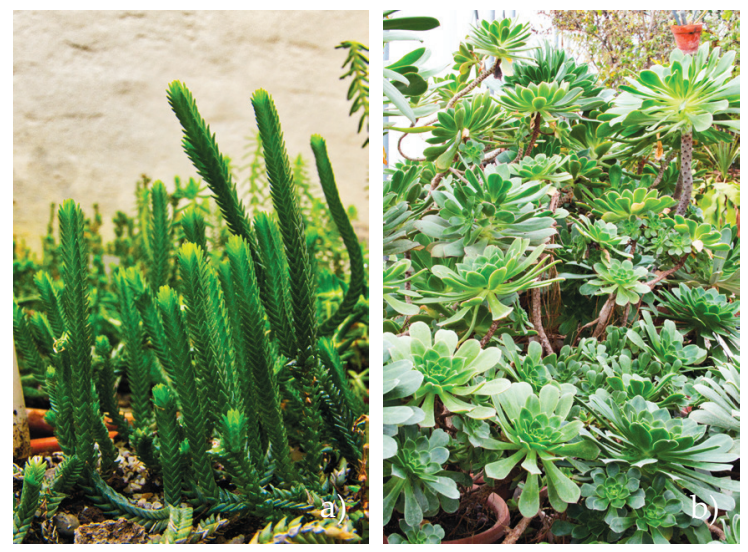

Fig. 5. Representatives of Crassulaceae family: a) Crassula muscosa L. var. musco$s a, b)$ representatives of genus Aeonium (with many species listed in EASIN and other invasive-plants databases)

S1. 5. Predstavnici porodice tustika (Crassulaceae): a) Crassula muscosa L. var. muscosa, b) predstavnici roda Aeonium (mnoge vrste također i na popisima invazivnih vrsta poput EASIN-a)
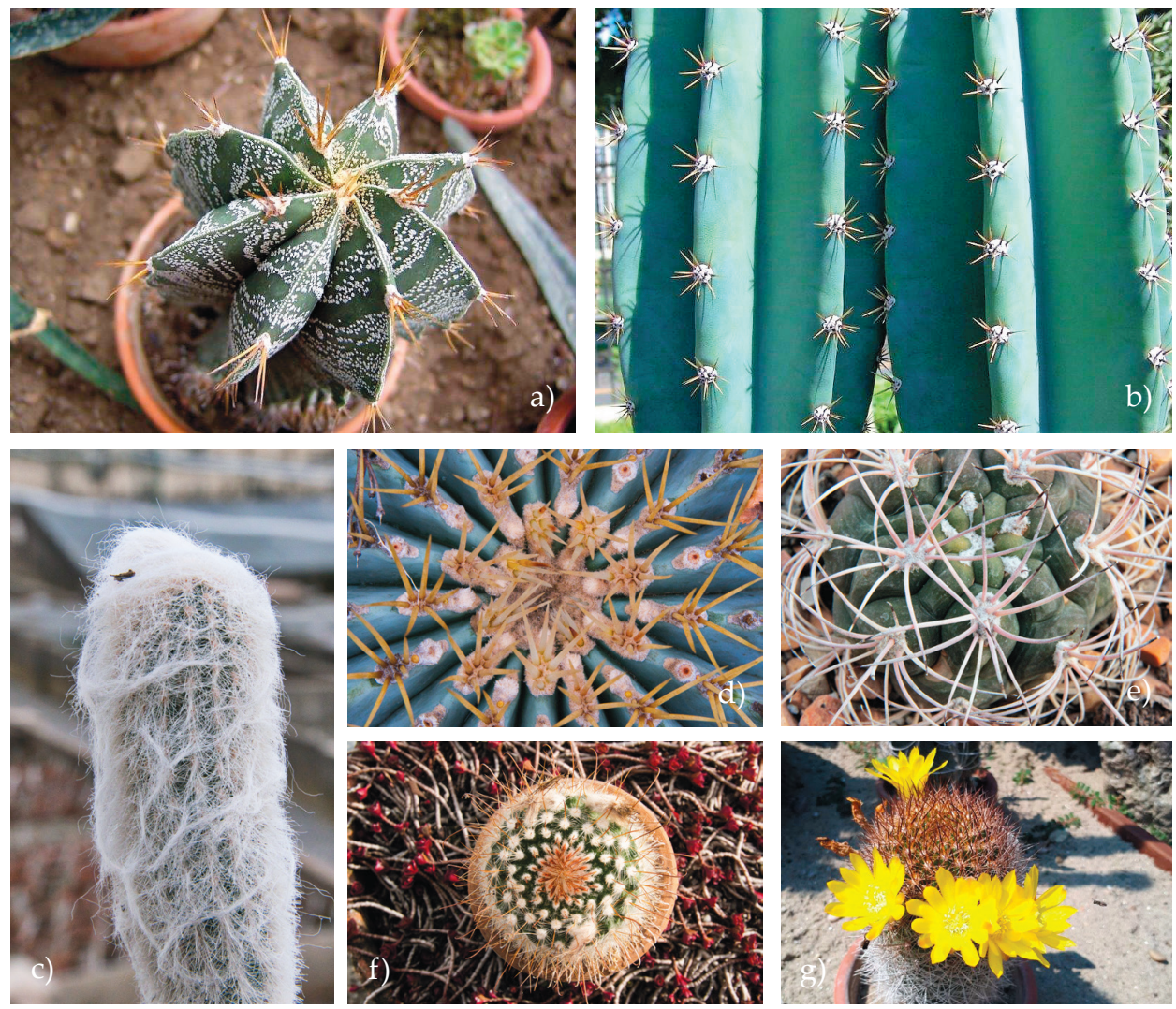

Fig. 6. Representatives of Cactaceae family: a) Astrophytum ornatum (DC.) Britton \& Rose, b) Cereus repandus (L.) Mill., c) Espostoa lanata (Kunth) Britton \& Rose, d) Ferocactus glaucescens (DC.) Britton \& Rose, e) Gymnocalycium saglionis (Cels) Britton \& Rose subsp. tilcarense (Backeb.) H.Till \& W.Till, f) Parodia mutabilis Backeb, g) Weingartia neocumingii Backeb." - all listed in CITES.

S1. 6. Predstavnici porodice kaktusa (Cactaceae): a) Astrophytum ornatum (DC.) Britton \& Rose, b) Cereus repandus (L.) Mill., c) Espostoa lanata (Kunth) Britton \& Rose, d) Ferocactus glaucescens (DC.) Britton \& Rose, e) Gymnocalycium saglionis (Cels) Britton \& Rose subsp. tilcarense (Backeb.) H.Till \& W.Till, f) Parodia mutabilis Backeb, g) Weingartia neocumingii Backeb." - sve prikazane vrste nalaze se na CITES-popisu. 


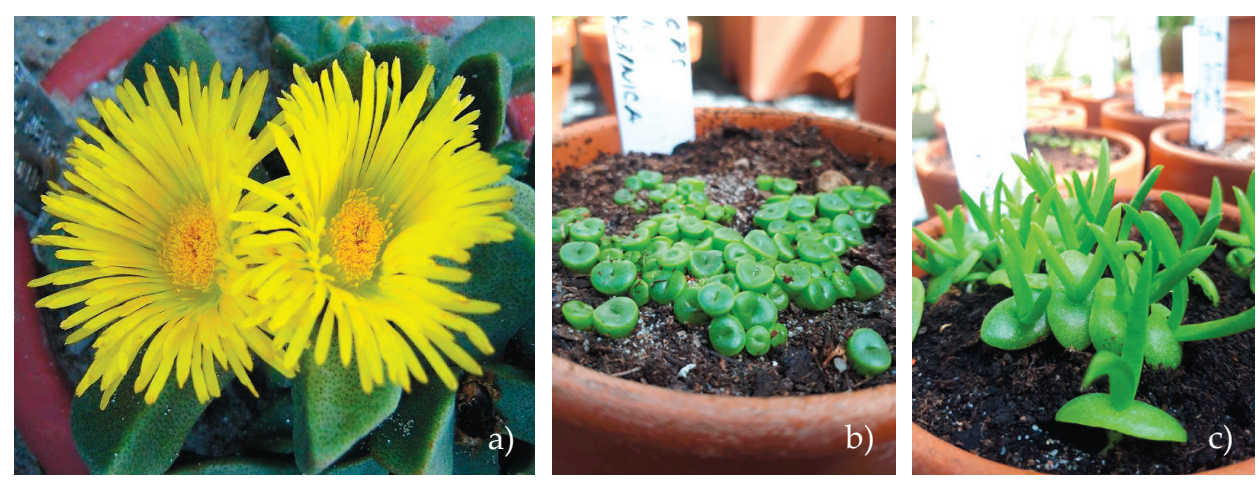

Fig. 7. Representatives of Aizoaceae family: a) Pleiospilos compactus Schwantes subsp. canus H.E.K. Hartmann \& Liede, b) Lithops lesliei (N.E. Br.) N.E.Br. subsp. lesliei (seedlings - new species in collection), c) Glotiphyllum longum (Haw.) N.E.Br. (seedlings - new species in collection).

Sl. 7. Predstavnici porodice čupavica (Aizoaceae): a) Pleiospilos compactus Schwantes subsp. canus H.E.K. Hartmann \& Liede, b) Lithops lesliei (N.E. Br.) N.E.Br. subsp. lesliei (seedlings - new species in collection), c) Glotiphyllum longum (Haw.) N.E.Br. (klijanci - nova vrsta u zbirci).
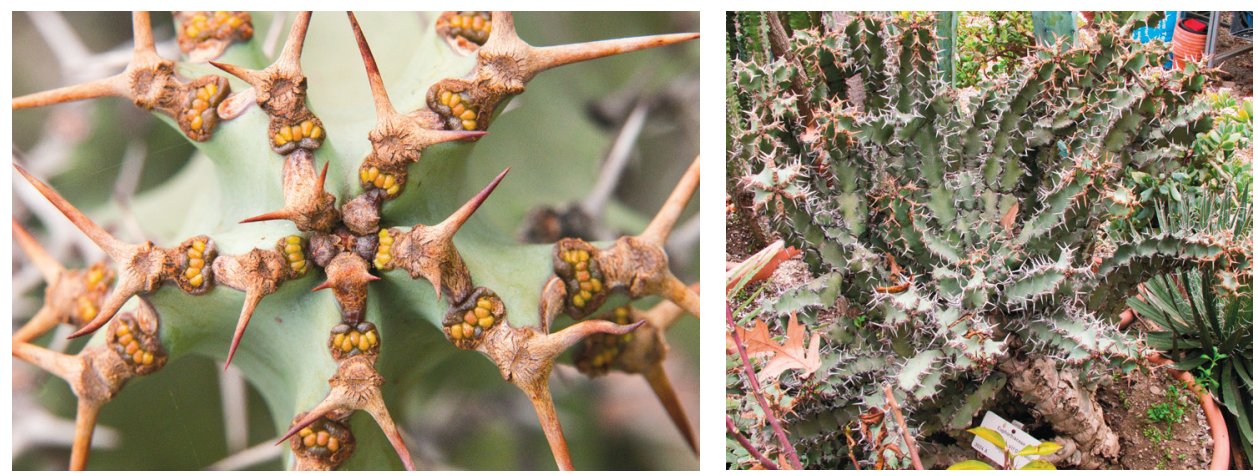

Fig. 8/8-1. Euphorbia virosa Willd. is listed in CITES

S1. 8./8-1. Euphorbia virosa Willd. nalazi se na CITES-popisu
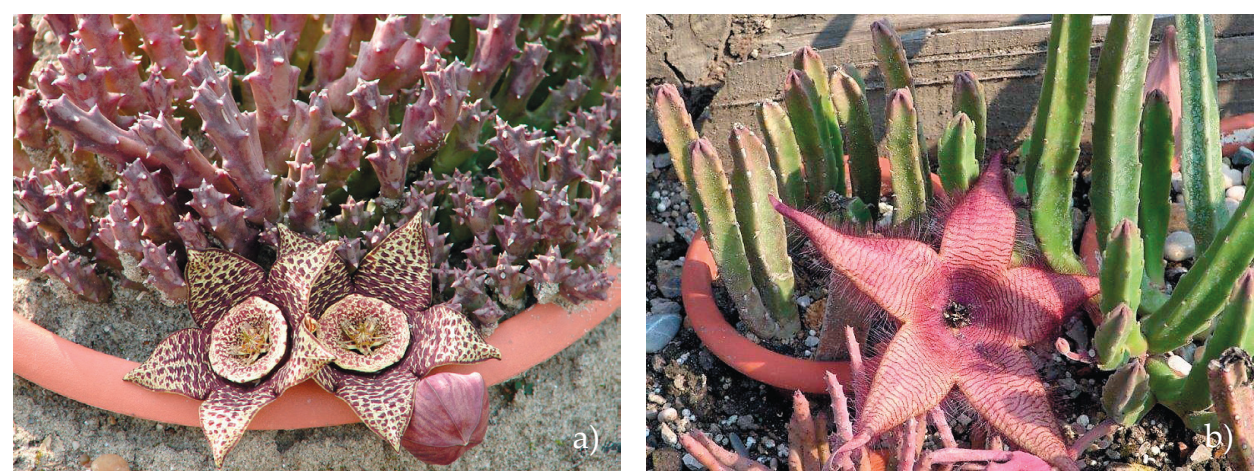

Fig. 9. Representatives of Asclepiadaceae family: a) Orbea variegata (L.) Haw., b) Stapelia grandiflora Masson.

S1. 9. Predstavnici porodice svilenica (Asclepiadaceae): a) Orbea variegata (L.) Haw., b) Stapelia grandiflora Masson. 


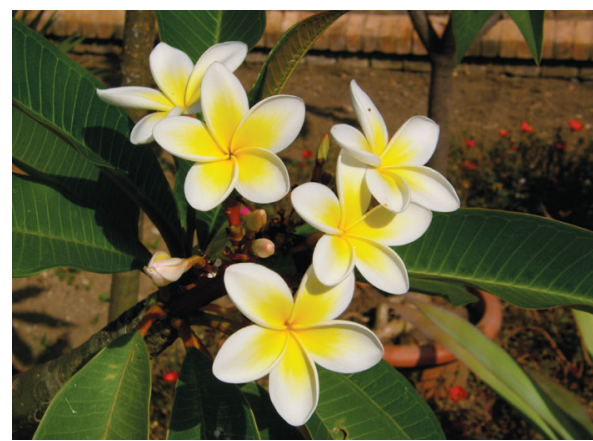

Fig. 10. Representative of the Apocynaceae family: Plumeria obtusa L. cult.

S1. 10. Predstavnik porodice zimzelena (Apocynaceae): Plumeria obtusa L. cult.

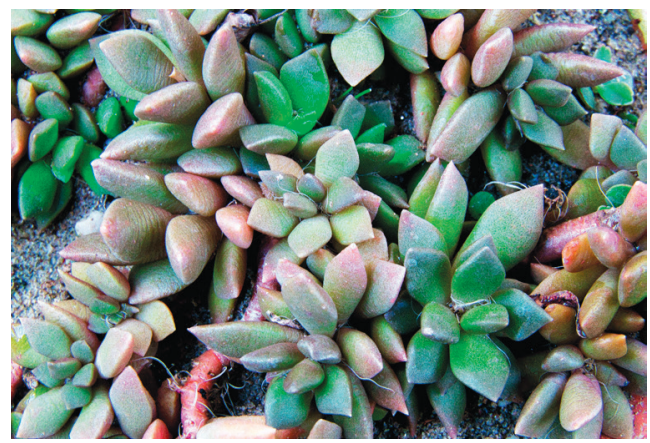

Fig. 11. Representative of the Portulacaceae family: Anacampseros rufescens (Haw.) Sweet, listed in CITES.

S1. 11. Predstavnik porodice tušakovica (Portulacaceae): Anacampseros rufescens (Haw.) Sweet, na CITES-popisu.
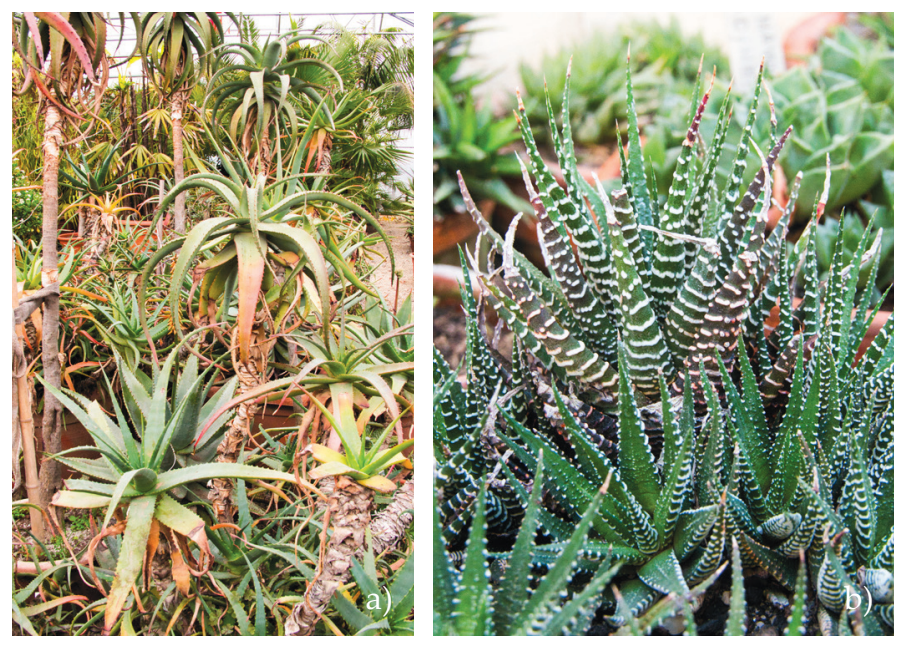

Fig. 12. Representatives of the Aloaceae family: a) all 18 species of the genus Aloe, held today in our cold glasshouse collection, are listed in CITES, b) Haworthia fasciata (Willd.) Haw.

S1. 12. Predstavnici porodice aloja (Aloaceae): a) svih 18 vrsta tipičnog roda (Aloe), koje danas imamo u zbirci, nalaze se na CITES-popisu, b) Haworthia fasciata (Willd.) Haw.
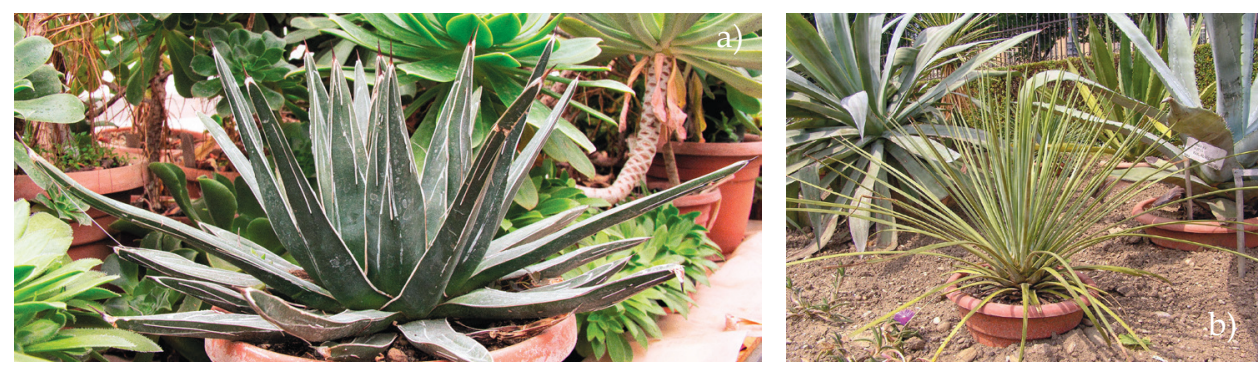

Fig. 13. Representatives of the Agavaceae family: a) Agave victoriae-reginae T. Moore, listed in CITES, b) Agave striata Zucc.

S1. 13. Predstavnice porodice agava (Agavaceae), a) Agave victoriae-reginae T. Moore, nalazi se na CITES-popisu, b) Agave striata Zucc. 
Tab. 2. Collection of the cold glasshouse between 1948 and 2017: recently non-existing plants.

\begin{tabular}{|c|c|c|c|c|}
\hline Scientific name & $\begin{array}{l}\text { City of plant } \\
\text { material origin }\end{array}$ & $\begin{array}{l}\text { Year of } \\
\text { ordering/ } \\
\text { purchasing }\end{array}$ & \begin{tabular}{|l|} 
Year when plant \\
was last recorded \\
in collection
\end{tabular} & Notes and remarks \\
\hline \multicolumn{5}{|l|}{ DICOTYLEDONS } \\
\hline \multicolumn{5}{|l|}{ Aizoaceae } \\
\hline Aloinopsis malherbei (L. Bolus) L. Bolus & Kirstenbosch & 1961 & 1963 & $\begin{array}{l}\text { arrived as Nananthus } \\
\text { malherbei }\end{array}$ \\
\hline Aloinopsis schooneesii L. Bolus & Kirstenbosch & 1961 & 1966 & $\begin{array}{l}\text { arrived as Nananthus } \\
\text { schooneesii; one of } 3 \\
\text { synonyms }\end{array}$ \\
\hline \multirow[t]{2}{*}{ Antegibbaeum fissoides (Haw.) C.Weber } & Barcelona & 1963 & 1977 & 2 synonyms \\
\hline & nn & unknown & 1975 & \\
\hline Antimima piscodora (L. Bolus) H. E. K. Hartmann & Gent & 1970 & 1971 & $\begin{array}{l}\text { arrived as Ruschia } \\
\text { piscodora; one of } 2 \\
\text { synonyms }\end{array}$ \\
\hline \multirow[t]{5}{*}{ Aptenia cordifolia (L.f.) Schwantes } & nn & 1952 & 1961 & 4 synonyms \\
\hline & Barcelona & 1971 & 2000 & \\
\hline & Karlsruhe & 1985 & 1990 & \\
\hline & Basel & 1986 & 1990 & \\
\hline & Prostejov & 1987 & 1990 & \\
\hline Argyroderma delaetii C.A.Maass & Hamburg & $1976 \& 1979$ & $1983 \& 1982$ & $\begin{array}{l}\text { arrived as A. roseum; one } \\
\text { of } 20+\text { synonyms }\end{array}$ \\
\hline Argyroderma fissum (Haw.) L.Bolus & Kirstenbosch & 1961 & 1972 & $\begin{array}{l}\text { arrived as } A \text {. brevipes; one } \\
\text { of } 8 \text { synonyms }\end{array}$ \\
\hline \multirow[t]{2}{*}{ Argyroderma framesii L.Bolus } & Kirstenbosch & 1957 & 1961 & 2 synonyms \\
\hline & Barcelona & 1957 & 1966 & \\
\hline Argyroderma pearsonii (N.E.Br.) Schwantes & Hamburg & 1976 & 1982 & 6 synonyms \\
\hline \multirow[t]{2}{*}{ Argyroderma testiculare (Aiton) N.E.Br. } & Muenchen & 1953 & 1961 & 2 synonyms \\
\hline & Kirstenbosch & 1961 & 1977 & \\
\hline Astridia velutina (L.Bolus) Dinter & Barcelona & 1959 & 1963 & 4 synonyms \\
\hline Bergeranthus addoensis L.Bolus & Kirstenbosch & 1970 & 1978 & \\
\hline \multirow{3}{*}{ Bergeranthus multiceps (Salm-Dyck) Schwantes } & $\mathrm{nn}$ & 1952 & 1990 & 4 synonyms \\
\hline & Barcelona & 1971 & 2000 & \\
\hline & Kirstenbosch & 1970 & 1980 & \\
\hline \multirow[t]{4}{*}{ Bergeranthus scapiger (Haw.) Schwantes } & Koeln & 1953 & 1961 & $\begin{array}{l}\text { syn. Mesembryanthemum } \\
\text { scapiger }\end{array}$ \\
\hline & Vienna & 1967 & 1970 & \\
\hline & nn & $\begin{array}{l}1952 \text { \& in } \\
\text { collection in } \\
1968\end{array}$ & $1977 \& 1970$ & \\
\hline & Gent & $1972 \& 1976$ & $1978 \& 1983$ & \\
\hline \multirow[t]{5}{*}{ Bergeranthus vespertinus (A. Berger) Schwantes } & nn & 1952 & no dana & 2 synonyms \\
\hline & Gent & $1966 \& 1972$ & $1980 \& 1972$ & \\
\hline & Berlin & 1979 & 1993 & \\
\hline & Basel & 1986 & 2000 & \\
\hline & Gent & 1966 & 1980 & arrived as B. jamesii \\
\hline Braunsia apiculata (Kensit) A.E. Murray & Kirstenbosch & 1961 & 1963 & $\begin{array}{l}\text { arrived as Echinus } \\
\text { apiculatus; one more } \\
\text { synonym }\end{array}$ \\
\hline Braunsia geminata (Haw.) L.Bolus & Barcelona & 1963 & 1968 & $\begin{array}{l}\text { arrived as Echinus } \\
\text { geminatus; one more } \\
\text { synonym }\end{array}$ \\
\hline Calamophyllum cylindricum (Haw.) Schwantes & Adelaide & 1968 & 1978 & \\
\hline \multirow[t]{2}{*}{ Carpanthea pomeridiana (L.) N.E. Br. } & Wroclaw & 1955 & no dana & \\
\hline & Basel & 1985 & 1985 & \\
\hline Carpobrotus edulis (L.) N.E.Br. & Vis & 1963 & 1964 & 3 synonyms \\
\hline Carpobrotus muirii (L.Bolus) L.Bolus & Kirstenbosch & 1961 & 1966 & \\
\hline \multirow[t]{3}{*}{ Carruanthus ringens (L.) Boom } & Barcelona & $1956 \& 1960$ & $1961 \& 1978$ & $\begin{array}{l}\text { arrived as C. caninus \& } \\
\text { arrived as Tischleria peersii }\end{array}$ \\
\hline & Berlin & $1960 \& 1973$ & $1983 \& 1980$ & $\begin{array}{l}\text { arrived as Tischleria peersii } \\
\text { \& arrived as C. caninus }\end{array}$ \\
\hline & Palermo & 1959 & 1963 & arrived as Tischleria peersii \\
\hline Cephalophyllum alstonii Marloth ex L.Bolus & Kirstenbosch & $1957 \& 1961$ & $1969 \& 1969$ & syn. C. franciscii \\
\hline Cephalophyllum caespitosum H.Hartmann & Kiel & 1962 & 1968 & $\begin{array}{l}\text { arrived as Cheiridopsis } \\
\text { cuprea; one of } 2 \text { synonyms }\end{array}$ \\
\hline Cephalophyllum loreum (L.) Schwantes & Barcelona & 1962 & 1975 & \\
\hline
\end{tabular}


S2 Sandev, D. et al.: Plethora of plants - collections of the Botanical Garden, Faculty of Science, University of Zagreb (2)

\begin{tabular}{|c|c|c|c|c|}
\hline Scientific name & $\begin{array}{l}\text { City of plant } \\
\text { material origin }\end{array}$ & $\begin{array}{l}\text { Year of } \\
\text { ordering/ } \\
\text { purchasing }\end{array}$ & $\begin{array}{l}\text { Year when plant } \\
\text { was last recorded } \\
\text { in collection }\end{array}$ & Notes and remarks \\
\hline Cephalophyllum parviflorum L.Bolus & Barcelona & $1960 \& 1962$ & $1961 \& 1980$ & \\
\hline Cephalophyllum pillansii L.Bolus & Kirstenbosch & 1971 & 1977 & $\begin{array}{l}\text { arrived as C. pillansii var. } \\
\text { grandiflorum }\end{array}$ \\
\hline \multirow{2}{*}{ Cephalophyllum purpureo-album (Haw.) Schwantes } & Gent & 1962 & 1972 & arrived as C. gracile \\
\hline & Adelaide & 1972 & 1977 & \\
\hline Cephalophyllum regale L.Bolus & Palermo & 1962 & 1964 & syn. C. vanheerdei \\
\hline Cephalophyllum rigidum L.Bolus. & Kirstenbosch & $1970 \& 1971$ & $1977 \& 1976$ & arrived as C. aureorubrum \\
\hline \multirow[t]{2}{*}{ Cephalophyllum tricolorum (Haw.) Schwantes } & Barcelona & 1959 & 1978 & \\
\hline & Adelaide & 1968 & 1978 & \\
\hline \multirow[t]{2}{*}{ Cerochlamys pachyphylla (L.Bolus) L.Bolus } & Barcelona & 1959 & 1961 & \\
\hline & Gent & 1965 & 1972 & \\
\hline Chasmatophyllum nelii Schwantes & Gent & 19701976 & $1988 \& 1981$ & \\
\hline \multirow[t]{3}{*}{ Chasmatophyllum willowmorense L.Bolus } & Gent & $\begin{array}{l}1962 \& 1963 \\
\& 1970\end{array}$ & $\begin{array}{l}1966 \& 1970 \& \\
1972\end{array}$ & \\
\hline & Kirstenbosch & 1961 & 1968 & \\
\hline & Wageningen & 1963 & 1969 & \\
\hline \multirow[t]{2}{*}{ Cheiridopsis denticulata (Haw.) N.E.Br. } & Kirstenbosch & 1961 & 1972 & arrived as $C$. candidissima \\
\hline & Kiel & 1972 & 1973 & \\
\hline \multirow[t]{3}{*}{ Cheiridopsis namaquensis (Sond.) H.E.K.Hartmann } & Barcelona & 1972 & 1982 & arrived as C. difformis \\
\hline & Kiel & 1972 & 1977 & arrived as C. marlothii \\
\hline & $\begin{array}{l}\text { Muenchen- } \\
\text { Nymphenburg }\end{array}$ & 1991 & 1993 & \\
\hline \multirow[t]{3}{*}{ Cheiridopsis pillansii L.Bolus } & Kiel & 1962 & 1966 & 2 synonyms \\
\hline & Barcelona & 1964 & 1966 & \\
\hline & Palermo & 1964 & 1970 & \\
\hline Cheiridopsis purpurea L.Bolus & Barcelona & 1964 & 1970 & syn. C. splendens \\
\hline \multirow[t]{4}{*}{ Cheiridopsis rostrata (L.) N.E.Br. } & Palermo & 1962 & 1980 & $\begin{array}{l}\text { arrived as C. carnea; one of } \\
15+\text { synonyms }\end{array}$ \\
\hline & Adelaide & 1968 & 1980 & \\
\hline & Barcelona & 1964 & 1983 & arrived as $C$. inspersa \\
\hline & Barcelona & 1962 & 1966 & arrived as C. bifida \\
\hline Cheiridopsis speciosa L.Bolus & Barcelona & 1962 & 1980 & arrived as C. comptonii \\
\hline \multirow[t]{4}{*}{ Cleretum bellidiforme (Burm. f.) G.D. Rowley } & Rotterdam & 1964 & 1964 & $\begin{array}{l}\text { arrived as Dorotheanthus } \\
\text { bellidiformis; one od } 10+ \\
\text { synonyms }\end{array}$ \\
\hline & Muenchen & 1972 & 1975 & \\
\hline & Essen & 1992 & 1993 & \\
\hline & $\begin{array}{l}\text { Muenchen- } \\
\text { Nymphenburg }\end{array}$ & 1992 & 1993 & \\
\hline \multirow[t]{5}{*}{$\begin{array}{l}\text { Conicosia pugioniformis (L.) N.E. Br. } \\
\text { subsp. pugioniformis }\end{array}$} & Kiel & 1996 & 1996 & $\begin{array}{l}\text { arrived as C. pugioniformis; } \\
\text { one of } 20+\text { synonyms }\end{array}$ \\
\hline & Karlsruhe & 1963 & 1968 & \\
\hline & Barcelona & 1962 & 1963 & arrived as C. communis \\
\hline & Berlin & 1967 & 1968 & \\
\hline & Kiel & $1996 \& 1998$ & $1996 \& 1998$ & \\
\hline \multirow[t]{2}{*}{ Conophytum bilobum (Marloth) N.E. Br. } & Monaco & $1992 \& 1993$ & 1993 & one of $20+$ synonyms \\
\hline & Berlin & 1966 & 1968 & $\begin{array}{l}\text { arrived as C. muscosipapil- } \\
\text { latum }\end{array}$ \\
\hline Conophytum ficiforme (Haw.) N.E.Br. & Vienna & 1964 & 1964 & $\begin{array}{l}\text { arrived as C. wiggettae; } \\
\text { from cuttings; syn. C. } \\
\text { assimile }\end{array}$ \\
\hline \multirow[t]{3}{*}{ Conophytum lydiae (H. Jacobsen) G.D. Rowley } & nn & 1951 & no data & $\begin{array}{l}\text { arrived as Ophthalmophyl- } \\
\text { lum lydiae; one of } 3 \\
\text { synonyms }\end{array}$ \\
\hline & Gent & 1971 & 1974 & \\
\hline & Kiel & 1996 & 1997 & \\
\hline Conophytum maughanii N.E. Br. subsp. maughanii & Nantes & 1992 & 2003 & $\begin{array}{l}\text { arrived as Ophthalmophyl- } \\
\text { lum maughanii; one of } 5 \\
\text { synonyms }\end{array}$ \\
\hline $\begin{array}{l}\text { Conophytum taylorianum (Dinter \& Schwantes) } \\
\text { N.E. Br. }\end{array}$ & Monaco & 2000 & 2003 & 5 synonyms \\
\hline $\begin{array}{l}\text { Conophytum taylorianum subsp. ernianum (Loesch } \\
\text { \& Tischer) de Boer ex S.A.Hammer }\end{array}$ & Muenster & 1968 & 1975 & arrived as C. ernianum \\
\hline \multirow{2}{*}{ Corpuscularia lehmannii (Eckl. \& Zeyh.) Schwantes } & nn & 1951 & 1980 & 2 synonyms \\
\hline & Napulj & 1960 & 1966 & \\
\hline
\end{tabular}




\begin{tabular}{|c|c|c|c|c|}
\hline Scientific name & $\begin{array}{l}\text { City of plant } \\
\text { material origin }\end{array}$ & $\begin{array}{l}\text { Year of } \\
\text { ordering/ } \\
\text { purchasing }\end{array}$ & $\begin{array}{l}\text { Year when plant } \\
\text { was last recorded } \\
\text { in collection }\end{array}$ & Notes and remarks \\
\hline Cryophytum guerichianum Schwantes & Muenchen & 1978 & 1980 & $\begin{array}{l}\text { arrived as Amoebophyllum } \\
\text { gurichianum; unresolved } \\
\text { name (The Plant List) }\end{array}$ \\
\hline Cylindrophyllum comptonii L.Bolus & Palermo & $1950 \& 1959$ & $1968 \& 1972$ & \\
\hline Cylindrophyllum tugwelliae L.Bolus & Kirstenbosch & 1970 & 1972 & \\
\hline \multirow[t]{3}{*}{ Delosperma aberdeenense (L.Bolus) L.Bolus } & Cantonspark & 1954 & 1961 & \\
\hline & nn & $\begin{array}{l}\text { in collection } \\
\text { in } 1963\end{array}$ & 1968 & \\
\hline & Berlin & $\begin{array}{l}1972 \& 1974 \\
\& 1978\end{array}$ & $\begin{array}{l}1978 \& 1979 \& \\
1982\end{array}$ & \\
\hline Delosperma abyssinicum Schwantes & $\mathrm{nn}$ & unknown & 1968 & \\
\hline \multirow[t]{4}{*}{$\begin{array}{l}\text { Delosperma brunnthaleri (A. Berger) Schwantes ex } \\
\text { H. Jacobsen }\end{array}$} & Vienna & 1961 & 1962 & $\begin{array}{l}\text { syn. Mesembryanthemum } \\
\text { brunnthaleri }\end{array}$ \\
\hline & $\mathrm{nn}$ & unknown & 1963 & \\
\hline & Gent & 1978 & 1979 & \\
\hline & Innsbruck & 2000 & 2003 & \\
\hline \multirow[t]{3}{*}{ Delosperma caespitosum L.Bolus } & $\mathrm{nn}$ & 1952 & 1971 & \\
\hline & Gent & 1970 & 1979 & \\
\hline & Berlin & $1972 \& 1976$ & $1979 \& 1979$ & \\
\hline Delosperma carolinense N.E.Br. & Gent & 1970 & 1979 & \\
\hline \multirow[t]{3}{*}{ Delosperma cooperi (Hook.f.) L. Bolus } & Gent & $1960 \& 1970$ & $1968 \& 1980$ & 2 synonyms \\
\hline & Berlin & $1974 \& 1979$ & $1978 \& 2000$ & \\
\hline & Kiel & 2003 & 2006 & \\
\hline Delosperma crassuloides (Haw.) L.Bolus & Berlin & $\begin{array}{l}19721978 \& \\
1979\end{array}$ & $\begin{array}{l}1979 \& 1990 \& \text { \& } \\
1990\end{array}$ & \\
\hline Delosperma davyi N.E. Br. & Berlin & $\begin{array}{l}1952 \& 1972 \\
\& 1979\end{array}$ & $\begin{array}{l}1961 \& 1979 \& \\
1979\end{array}$ & \\
\hline Delosperma echinatum (Lam.) Schwantes & Szeged & 1960 & 1975 & $\begin{array}{l}5 \text { synonyms; unresolved } \\
\text { name (the Plant List) }\end{array}$ \\
\hline \multirow[t]{2}{*}{ Delosperma ecklonis (Salm-Dyck) Schwantes } & Lyon & 1953 & no data & 2 synonyms \\
\hline & Vienna & 1961 & 1966 & \\
\hline Delosperma framesii L.Bolus & Berlin & 1979 & 1983 & \\
\hline \multirow[t]{2}{*}{ Delosperma hirtum (N.E.Br.) Schwantes } & Gent & 1970 & 1979 & \\
\hline & Berlin & 1978 & 1979 & \\
\hline Delosperma lavisiae L.Bolus & Gent & 1970 & 1979 & \\
\hline \multirow[t]{2}{*}{ Delosperma macellum N.E. Br. } & Gent & 1970 & 1972 & \\
\hline & Berlin & $1973 \& 1974$ & $1983 \& 1982$ & \\
\hline \multirow[t]{3}{*}{ Delosperma mahonii N.E. Br. } & Essen & 1970 & 1979 & \\
\hline & Gent & 1970 & 1979 & \\
\hline & Berlin & 1979 & 1983 & \\
\hline Delosperma multiflora L. Bolus & Gent & 1976 & 1980 & $\begin{array}{l}\text { arrived as false species; } \\
\text { never determinated-sold }\end{array}$ \\
\hline \multirow[t]{2}{*}{ Delosperma nakurense (Engl.) Herre } & Muenchen & 1966 & 1968 & \\
\hline & Berlin & 1973 & 1982 & \\
\hline Delosperma prasinum L. Bolus & Berlin & 1973 & 1975 & \\
\hline \multirow[t]{2}{*}{ Delosperma robustum L. Bolus } & Gent & 1970 & 2000 & \\
\hline & Berlin & $1972 \& 1978$ & $1978 \& 1990$ & \\
\hline Delosperma rogersii L. Bolus & Berlin & 1979 & 1979 & \\
\hline \multirow[t]{4}{*}{ Delosperma steytlerae L. Bolus } & Karlsruhe & 1961 & 1961 & \\
\hline & Vienna & 1961 & 1963 & \\
\hline & nn & unknown & 1966 & \\
\hline & Berlin & $1972 \& 1979$ & $1972 \& 1983$ & \\
\hline Delosperma sutherlandii (Hook. f.) N.E. Br. & Monaco & 2002 & 2008 & $\begin{array}{l}\text { syn. Mesembryanthemum } \\
\text { sutherlandii }\end{array}$ \\
\hline Delosperma tradescantioides L. Bolus & Nancy & 1971 & 1978 & \\
\hline Delosperma wethamae L. Bolus & Gent & 1970 & 1972 & \\
\hline \multirow[t]{2}{*}{ Delosperma zoeae L. Bolus } & Muenchen & 1968 & 1973 & \\
\hline & Berlin & $\begin{array}{l}1968 \& 1978 \\
\& 1979\end{array}$ & $\begin{array}{l}1972 \& \text { \& } 1979 \& \\
1979\end{array}$ & \\
\hline \multirow[t]{2}{*}{ Dicrocaulon spissum N.E. Br. } & Nantes & 1996 & 2003 & \\
\hline & Potsdam & 1996 & 2000 & \\
\hline \multirow[t]{2}{*}{ Diplosoma retroversum (Kensit) Schwantes } & Barcelona & $1958 \& 1959$ & $1983 \& 1982$ & 4 synonyms \\
\hline & Kirstenbosch & 1962 & 1963 & $\begin{array}{l}\text { arrived as Maughania } \\
\text { luckhoffii }\end{array}$ \\
\hline
\end{tabular}


S4 Sandev, D. et al.: Plethora of plants - collections of the Botanical Garden, Faculty of Science, University of Zagreb (2)

\begin{tabular}{|c|c|c|c|c|}
\hline Scientific name & $\begin{array}{l}\text { City of plant } \\
\text { material origin }\end{array}$ & $\begin{array}{l}\text { Year of } \\
\text { ordering/ } \\
\text { purchasing }\end{array}$ & $\begin{array}{l}\text { Year when plant } \\
\text { was last recorded } \\
\text { in collection }\end{array}$ & Notes and remarks \\
\hline \multirow[t]{2}{*}{ Disphyma crassifolium (L.) L.Bolus } & Berlin & 1972 & 1979 & $\begin{array}{l}\text { syn. Mesembryanthemum } \\
\text { crassifolium }\end{array}$ \\
\hline & Barcelona & 1971 & 1975 & \\
\hline $\begin{array}{l}\text { Dorotheanthus bellidiformis (Burm.f.) N.E.Br. } \\
\text { subsp. bellidiformis N.E. Br. }\end{array}$ & Kirstenbosch & 1961 & 1961 & $\begin{array}{l}\text { arrived as D. bellidiformis } \\
\text { var. taldanhensis }\end{array}$ \\
\hline Dorotheanthus oculatus N.E. Br. & Leipzig & 1993 & 1993 & 3 synonyms \\
\hline Drosanthemum candens (Haw.) Schwantes & Berlin & 1979 & 1990 & $\begin{array}{l}\text { syn. Mesembryanthemum } \\
\text { candens }\end{array}$ \\
\hline Drosanthemum floribundum (Haw.) Schwantes & Muenchen & 1985 & 2000 & 4 synonyms \\
\hline Drosanthemum framesii L. Bolus & Berlin & 1979 & 1993 & \\
\hline Drosanthemum hispidum (L.) Schwantes & Basel & 1985 & 1985 & $\begin{array}{l}\text { syn. Mesembryanthemum } \\
\text { hispidum }\end{array}$ \\
\hline Drosanthemum latipetalum L. Bolus & $\mathrm{nn}$ & 1952 & 1961 & \\
\hline Drosanthemum pulchellum L. Bolus & Berlin & $\begin{array}{l}1966 \& 1974 \\
\& 1979\end{array}$ & $\begin{array}{l}1978 \& 1978 \& \\
1990\end{array}$ & \\
\hline \multirow[t]{2}{*}{ Drosanthemum schoenlandianum L. Bolus } & Gent & 1966 & 1972 & $\begin{array}{l}\text { syn. Mesembryanthemum } \\
\text { schoenlandianum }\end{array}$ \\
\hline & $\mathrm{nn}$ & unknown & 1972 & \\
\hline $\begin{array}{l}\text { Ebracteola derenbergiana (Dinter) Dinter \& } \\
\text { Schwantes }\end{array}$ & Kirstenbosch & 1964 & 1968 & $\begin{array}{l}\text { arrived as Ruschia } \\
\text { derenbergiana; one of } 10+ \\
\text { synonyms }\end{array}$ \\
\hline $\begin{array}{l}\text { Ebracteola montis-moltkei (Dinter) Dinter \& } \\
\text { Schwantes }\end{array}$ & Kiel & 1955 & 1963 & 2 synonyms \\
\hline Erepsia heteropetala (Haw.) Schwantes & Berlin & 1965 & 1971 & $\begin{array}{l}\text { syn. Mesembryanthemum } \\
\text { heteropetalum }\end{array}$ \\
\hline Erepsia inclaudens (Haw.) Schwantes & Pecs & 2005 & 2010 & \\
\hline \multirow[t]{6}{*}{ Faucaria bosscheana (A.Berger) Schwantes } & Basel & $1953 \& 1961$ & $1961 \& 1968$ & 8 synonyms \\
\hline & Palermo & 1962 & 1978 & \\
\hline & Berlin & 1971 & 1980 & \\
\hline & Duisburg & $1990 \& 1993$ & $1993 \& 1995$ & \\
\hline & Innsbruck & 2008 & 2016 & \\
\hline & Berlin & 1971 & 1973 & arrived as F. paucidens \\
\hline \multirow[t]{3}{*}{ Faucaria britteniae L. Bolus } & Berlin & $1971 \& 1973$ & $1980 \& 1980$ & $\begin{array}{l}\text { arrived as F. grandis; one } \\
\text { of } 5 \text { synonyms }\end{array}$ \\
\hline & Duisburg & 1990 & 1990 & \\
\hline & Innsbruck & 2008 & 2016 & \\
\hline \multirow[t]{3}{*}{ Faucaria felina (L.) Schwantes } & Nantes & 1966 & 1983 & $\begin{array}{l}\text { arrived as } F \text {. lupina; one of } \\
6 \text { synonyms }\end{array}$ \\
\hline & Berlin & $1971 \& 1973$ & $2000 \& 1980$ & \\
\hline & Basel & 1986 & 1990 & \\
\hline Faucaria tigrina (Haw.) Schwantes & Innsbruck & 2004 & 2016 & 3 synonyms \\
\hline \multirow{6}{*}{ Faucaria tuberculosa (Rolfe) Schwantes } & Gent & $1961 \& 1970$ & no data \& 1978 & 2 synonyms \\
\hline & Basel & 1961 & 1961 & \\
\hline & Palermo & $1961 \& 1964$ & $1968 \& 1978$ & \\
\hline & Berlin & 1961 & 1971 & \\
\hline & $\begin{array}{l}\text { Muenchen- } \\
\text { Nymphenburg }\end{array}$ & 1991 & 2000 & \\
\hline & Innsbruck & 2004 & 2006 & \\
\hline $\begin{array}{l}\text { Fenestraria rhopalophylla (Schltdl. \& Diels) N.E.Br. } \\
\text { subsp. aurantiaca (N.E. Br.) H. E. K. Hartmann }\end{array}$ & Dresden & $1996 \& 2000$ & $1997 \& 2001$ & arrived as F. aurantiaca \\
\hline Gibbaeum angulipes (L. Bolus) N.E. Br. & Basel & 1994 & 2000 & $\begin{array}{l}\text { syn. Mesembryanthemum } \\
\text { angulipes }\end{array}$ \\
\hline Gibbaeum dispar N.E.Br. & Barcelona & 1962 & 1968 & \\
\hline Gibbaeum gibbosum (Haw.) N.E.Br. & Kirstenbosch & 1964 & 1975 & 2 synonyms \\
\hline \multirow[t]{2}{*}{ Gibbaeum pachypodium L. Bolus } & Gent & 1964 & 1969 & \\
\hline & Kirstenbosch & 1964 & 1968 & \\
\hline \multirow[t]{5}{*}{ Gibbaeum petrense (N.E. Br.) Tischler } & Kirstenbosch & 1964 & 1970 & 2 synonyms \\
\hline & Nantes & 1996. & 1997 & \\
\hline & Kirstenbosch & $\begin{array}{l}1961 \& 1964 \\
\& 1966\end{array}$ & $\begin{array}{l}1966 \& 1968 \& \\
1967\end{array}$ & arrived as $G$. haagei \\
\hline & Barcelona & 1963 & 1968 & \\
\hline & Gent & 1970 & 1983 & \\
\hline Gibbaeum velutinum (L. Bolus) Schwantes & Kiel & $\begin{array}{l}1955 \& 1962 \\
\& 1964\end{array}$ & $\begin{array}{l}\text { no data \& } 1966 \\
\& 1978\end{array}$ & $\begin{array}{l}\text { arrived as G. schwantesii; } \\
\text { one of } 2 \text { synonyms }\end{array}$ \\
\hline
\end{tabular}




\begin{tabular}{|c|c|c|c|c|}
\hline Scientific name & $\begin{array}{l}\text { City of plant } \\
\text { material origin }\end{array}$ & $\begin{array}{l}\text { Year of } \\
\text { ordering/ } \\
\text { purchasing }\end{array}$ & $\begin{array}{l}\text { Year when plant } \\
\text { was last recorded } \\
\text { in collection }\end{array}$ & Notes and remarks \\
\hline & Frankfurt & 1955 & 1961 & \\
\hline & Nantes & 1995 & 2000 & \\
\hline & Groningen & 1961 & 1983 & \\
\hline & Kirstenbosch & $1961 \& 1966$ & $1978 \& 1971$ & \\
\hline & Gent & 1963 & 1966 & \\
\hline & Kiel & $1964 \& 1966$ & $1967 \& 1970$ & \\
\hline & Barcelona & 1972 & 1974 & \\
\hline & Graz & 1991 & 2000 & \\
\hline Glottiphyllum album L. Bolus ex Jacobsen & Berlin & 1961 & 1979 & \\
\hline \multirow[t]{3}{*}{ Glottiphyllum cruciatum (Haw.) N.E.Br. } & nn & 1951 & 1968 & $\begin{array}{l}\text { arrived as G. praepingue; } \\
\text { one of } 10+\text { synonyms }\end{array}$ \\
\hline & nn & 1952 & 1961 & \\
\hline & Gent & 1970 & 1979 & \\
\hline \multirow[t]{8}{*}{ Glottiphyllum depressum (Haw.) N.E. Br. } & Genova & 1960 & 1966 & $\begin{array}{l}\text { arrived as G. fragrans; one } \\
\text { of } 20+\text { synonyms }\end{array}$ \\
\hline & nn & $\begin{array}{l}1951 \& \text { in } \\
\text { collection in } \\
1968 \text { \& in } \\
\text { collection in } \\
1982\end{array}$ & $\begin{array}{l}\text { no data \& } 1981 \\
\& 2002\end{array}$ & \\
\hline & Berlin & 1973 & 1979 & \\
\hline & Gent & 1979 & 1980 & \\
\hline & nn & $\begin{array}{l}1952 \text { \& in } \\
\text { collection in } \\
1963\end{array}$ & $1961 \& 1989$ & arrived as G. marlothii \\
\hline & $\begin{array}{l}\text { Muenchen- } \\
\text { Nymphenburg }\end{array}$ & 1991 & 1993 & \\
\hline & Palermo & $1993 \& 1994$ & $2002 \& 2000$ & \\
\hline & nn & \begin{tabular}{|l|}
$1951 \&$ in \\
collection in \\
1980
\end{tabular} & 1993 \& 1989 & arrived as G. depressum \\
\hline \multirow[t]{3}{*}{ Glottiphyllum difforme (L.) N.E.Br. } & Vienna & 1957 & 1978 & $\begin{array}{l}\text { arrived as G. semicylindri- } \\
\text { cum }\end{array}$ \\
\hline & nn & $\begin{array}{l}1952 \text { in } \\
\text { collection in } \\
1963\end{array}$ & $1961 \& 1979$ & \\
\hline & Berlin & 1971 & 1979 & \\
\hline \multirow[t]{7}{*}{ Glottiphyllum linguiforme (L.) N.E. Br. } & Kirstenbosch & 1957 & 1979 & 6 synonyms \\
\hline & nn & $\begin{array}{l}\text { in collection } \\
\text { in } 1973\end{array}$ & 1979 & \\
\hline & Latte & 1990 & 2000 & \\
\hline & Dijon & 1991 & 1995 & \\
\hline & Groningen & 1960 & 1961 & arrived as G. latifolium \\
\hline & $\mathrm{nn}$ & $\begin{array}{l}\text { in collection } \\
\text { in } 1963\end{array}$ & 1979 & \\
\hline & Gent & 1979 & 1980 & \\
\hline \multirow[t]{6}{*}{ Glottiphyllum longum (Haw.) N.E.Br. } & nn & \begin{tabular}{|l|}
$1952 \&$ in \\
collection in \\
1961
\end{tabular} & no data \& 1966 & $\begin{array}{l}\text { arrived as G. latum; one of } \\
15+\text { synonyms }\end{array}$ \\
\hline & nn & 1950 & 1961 & arrived as G. longum \\
\hline & Kirstenbosch & 1957 & 1979 & \\
\hline & Berlin & 1971 & 1979 & \\
\hline & Muenchen & 1970 & 1980 & \\
\hline & Essen & 2006 & 2010 & \\
\hline \multirow[t]{3}{*}{ Glottiphyllum neilii N.E. Br. } & nn & \begin{tabular}{|l|}
$1951 \& 1952 \&$ \\
in collection \\
in 1961
\end{tabular} & $\begin{array}{l}\text { no data \& no } \\
\text { data \& } 1979\end{array}$ & \\
\hline & Berlin & 1979 & 1979 & \\
\hline & Debrecen & 1980 & no data & \\
\hline \multirow[t]{2}{*}{ Glottiphyllum regium N.E.Br. } & Kirstenbosch & 1967 & 1980 & syn. G. compressum \\
\hline & nn & unknown & no data & \\
\hline \multirow[t]{2}{*}{ Glottiphyllum suave N.E.Br. } & nn & 1951 & no data & arrived as G. herrei \\
\hline & Kiel & 1958 & 1961 & \\
\hline Glottiphyllum surrectum (Haw.) L.Bolus & Kirstenbosch & 1964 & 1979 & $\begin{array}{l}\text { syn. Mesembryanthemum } \\
\text { surrectum }\end{array}$ \\
\hline
\end{tabular}


S6 Sandev, D. et al.: Plethora of plants - collections of the Botanical Garden, Faculty of Science, University of Zagreb (2)

\begin{tabular}{|c|c|c|c|c|}
\hline Scientific name & $\begin{array}{l}\text { City of plant } \\
\text { material origin }\end{array}$ & $\begin{array}{l}\text { Year of } \\
\text { ordering/ } \\
\text { purchasing }\end{array}$ & $\begin{array}{l}\text { Year when plant } \\
\text { was last recorded } \\
\text { in collection }\end{array}$ & Notes and remarks \\
\hline & Berlin & 1971 & 1979 & \\
\hline Hereroa acuminata L. Bolus & Berlin & 1967 & 1968 & \\
\hline Hereroa calycina L. Bolus & Bochum & 2003 & 2010 & \\
\hline \multirow[t]{2}{*}{ Hereroa granulata (N.E. Br.) Dinter \& Schwantes } & Muenchen & 1966 & 1971 & $\begin{array}{l}\text { syn. Mesembryanthemum } \\
\text { granulatum }\end{array}$ \\
\hline & Gent & 1970 & 1983 & \\
\hline \multirow[t]{2}{*}{$\begin{array}{l}\text { Hereroa hesperantha (Dinter \& A. Berger) Dinter } \\
\text { \& Schwantes }\end{array}$} & nn & $\begin{array}{l}\text { in collection } \\
\text { in } 1961\end{array}$ & 1971 & 2 synonyms \\
\hline & Berlin & 1967 & 1971 & \\
\hline \multirow[t]{2}{*}{ Hereroa incurva L. Bolus } & nn & $\begin{array}{l}1951 \& \text { in } \\
\text { collection in } \\
1968\end{array}$ & 1968 \& 1975 & \\
\hline & Gent & 1976 & 1980 & \\
\hline \multirow[t]{3}{*}{ Hereroa muirii L. Bolus } & nn & $1951 \& 1964$ & $1961 \& 1980$ & \\
\hline & Barcelona & 1964 & 1968 & \\
\hline & Prostejov & 1987 & 2000 & \\
\hline \multirow[t]{2}{*}{ Hereroa nelii Schwantes } & nn & 1953 & 1961 & \\
\hline & Berlin & $1973 \& 1979$ & $1980 \& 1983$ & \\
\hline \multirow{2}{*}{$\begin{array}{l}\text { Hereroa puttkameriana (Dinter \& A. Berger) Dinter } \\
\text { \& Schwantes }\end{array}$} & Muenchen & 1970 & 1977 & $\begin{array}{l}\text { syn. Mesembryanthemum } \\
\text { puttkamerianum }\end{array}$ \\
\hline & Berlin & $1971 \& 1979$ & $1971 \& 1993$ & \\
\hline \multirow[t]{3}{*}{$\begin{array}{l}\text { Hereroa rehneltiana (A. Berger) Dinter \& } \\
\text { Schwantes }\end{array}$} & nn & 1952 & 1971 & $\begin{array}{l}\text { syn. Mesembryanthemum } \\
\text { rehneltianum }\end{array}$ \\
\hline & Berlin & 1958 & 1968 & \\
\hline & nn & unknown & 1968 & \\
\hline Hereroa stanfordiae L. Bolus & Berlin & 1967 & 1971 & \\
\hline Hereroa wilmaniae L. Bolus & Gent & 1970 & 1975 & 3 synonyms \\
\hline Ihlenfeldtia vanzylii (L. Bolus) H. E. K. Hartmann & Lisbon & $1954 \& 1955$ & $1961 \& 1961$ & $\begin{array}{l}\text { arrived as Cheiridopsis } \\
\text { vanzijlii; one of } 3 \\
\text { synonyms }\end{array}$ \\
\hline Jacobsenia kolbei (L. Bolus) L. Bolus \& Schwantes & Kirstenbosch & $\begin{array}{l}1958 \& 1961 \\
\& 1976\end{array}$ & $\begin{array}{l}1968 \& 1968 \& \\
1978\end{array}$ & $\begin{array}{l}\text { syn. Mesembryanthemum } \\
\text { kolbei }\end{array}$ \\
\hline Jordaaniella cuprea H.E.K. Hartm. & Barcelona & 1969 & 1973 & $\begin{array}{l}\text { arrived as Cephalophyllum } \\
\text { cupreum }\end{array}$ \\
\hline Juttadinteria ausensis Schwantes & Dresden & 1997 & 2006 & arrived as J. suavissima \\
\hline Juttadinteria deserticola (Marloth) Schwantes & Kiel & 1955 & no data & $\begin{array}{l}\text { syn. Mesembryanthemum } \\
\text { deserticola }\end{array}$ \\
\hline Juttadinteria simpsonii (Dinter) Schwantes & Barcelona & $\begin{array}{l}\text { in collection } \\
\text { in } 1967\end{array}$ & 1968 & 2 synonyms \\
\hline Kewa bowkeriana (Sond.) Christenh. & Mozambique & 1973 & 1977 & $\begin{array}{l}\text { arrived as Hypertelis } \\
\text { bowkeriana; one of } 2 \\
\text { synonyms; from natural } \\
\text { habitat }\end{array}$ \\
\hline Khadia acutipetala (N.E. Br.) N.E. Br. & Barcelona & $1963 \& 1964$ & no data \& 1980 & $\begin{array}{l}\text { syn. Mesembryanthemum } \\
\text { acutipetalum }\end{array}$ \\
\hline Lampranthus amoenus N.E. Br. & Marburg & 1953 & 1966 & $\begin{array}{l}\text { syn. Mesembryanthemum } \\
\text { amoenum }\end{array}$ \\
\hline Lampranthus aureus (L.) N.E. Br. & Halle & 1956 & 1963 & $\begin{array}{l}\text { syn. Mesembryanthemum } \\
\text { aureum }\end{array}$ \\
\hline Lampranthus bicolor (L.) N.E. Br. & Berlin & 1973 & 1979 & $\begin{array}{l}\text { syn. Mesembryanthemum } \\
\text { bicolor }\end{array}$ \\
\hline Lampranthus blandus (Haw.) Schwantes & Gent & 1966 & 1968 & $\begin{array}{l}\text { syn. Mesembryanthemum } \\
\text { blandum }\end{array}$ \\
\hline Lampranthus brownii (Hook. f.) N.E. Br. & nn & unknown & 1966 & $\begin{array}{l}\text { syn. Mesembryanthemum } \\
\text { brownii }\end{array}$ \\
\hline Lampranthus conspicuus (Haw.) N.E. Br. & Stockholm & unknown & 1961 & $\begin{array}{l}\text { syn. Mesembryanthemum } \\
\text { conspicuum }\end{array}$ \\
\hline \multirow[t]{2}{*}{ Lampranthus falcatus (L.) N.E. Br. } & Berlin & 1971 & 1972 & 2 synonyms \\
\hline & Barcelona & 1981 & 1990 & \\
\hline Lampranthus glomeratus (L.) N.E. Br. & Barcelona & 1968 & 1979 & $\begin{array}{l}\text { syn. Mesembryanthemum } \\
\text { glomeratum }\end{array}$ \\
\hline Lampranthus haworthii N.E. Br. & Wageningen & 1959 & 1966 & $\begin{array}{l}\text { arrived as Erepsia } \\
\text { haworthii; one of } 2 \\
\text { synonyms }\end{array}$ \\
\hline
\end{tabular}




\begin{tabular}{|c|c|c|c|c|}
\hline Scientific name & $\begin{array}{l}\text { City of plant } \\
\text { material origin }\end{array}$ & $\begin{array}{l}\text { Year of } \\
\text { ordering/ } \\
\text { purchasing }\end{array}$ & $\begin{array}{l}\text { Year when plant } \\
\text { was last recorded } \\
\text { in collection }\end{array}$ & Notes and remarks \\
\hline \multirow[t]{2}{*}{ Lampranthus inaequalis (Haw.) N.E. Br. } & Berlin & $1966 \& 1967$ & $1968 \& 1968$ & 2 synonyms \\
\hline & nn & 1973 & 1977 & \\
\hline Lampranthus productus (Haw.) N.E. Br. & Muenchen & 1973 & 1978 & $\begin{array}{l}\text { syn. Mesembryanthemum } \\
\text { productum }\end{array}$ \\
\hline \multirow[t]{3}{*}{ Lampranthus roseus (Willd.) Schwantes } & Vienna & 1961 & 1961 & $\begin{array}{l}\text { syn. Mesembryanthemum } \\
\text { roseum }\end{array}$ \\
\hline & Kirstenbosch & 1970 & 1978 & \\
\hline & Muenchen & $1970 \& 1972$ & $1978 \& 1978$ & \\
\hline Lampranthus sociorum (L. Bolus) N.E. Br. & Berlin & 1971 & 1971 & $\begin{array}{l}\text { syn. Mesembryanthemum } \\
\text { sociorum }\end{array}$ \\
\hline Lampranthus variabilis (Haw.) N.E. Br. & Berlin & 1979 & 1989 & $\begin{array}{l}\text { syn. Mesembryanthemum } \\
\text { variabile }\end{array}$ \\
\hline \multirow[t]{2}{*}{ Lampranthus watermeyeri (L. Bolus) N.E. Br. } & Gent & $\begin{array}{l}1966 \& 1967 \\
\& 1970\end{array}$ & $\begin{array}{l}1968 \& 1971 \& \\
1979\end{array}$ & $\begin{array}{l}\text { syn. Mesembryanthemum } \\
\text { watermeyeri }\end{array}$ \\
\hline & $\mathrm{nn}$ & unknown & 1968 & \\
\hline \multirow[t]{2}{*}{ Lampranthus zeyheri (Salm-Dyck) N.E. Br. } & Berlin & 1966 & 1968 & $\begin{array}{l}\text { syn. Mesembryanthemum } \\
\text { zeyheri }\end{array}$ \\
\hline & $\mathrm{nn}$ & unknown & 1968 & \\
\hline \multirow[t]{2}{*}{$\begin{array}{l}\text { Lapidaria margaretae (Schwantes) Dinter \& } \\
\text { Schwantes }\end{array}$} & Dresden & 1996 \& 1998 & $1997 \& 2000$ & 2 synonyms \\
\hline & Innsbruck & 1998 & no data & \\
\hline Leipoldtia schultzei (Schltr. \& Diels) Friedrich & Barcelona & 1959 & 1966 & $\begin{array}{l}\text { arrived as L. nelii; one of } 3 \\
\text { synonyms }\end{array}$ \\
\hline \multirow[t]{3}{*}{ Lithops aucampiae L. Bolus } & Muenchen & 1953 & no data & syn. L. koelemanii \\
\hline & Muenster & 1968 & 1971 & \\
\hline & Gent & 1967 & 1971 & \\
\hline \multirow[t]{4}{*}{ Lithops bella N.E. Br. } & Kiel & 1958 & 1961 & 2 synonyms \\
\hline & Gent & 1965 & 1968 & \\
\hline & Muenster & 1968 & 1971 & \\
\hline & Graz & 1991 & no data & \\
\hline \multirow[t]{5}{*}{ Lithops bromfieldii L. Bolus } & Kirstenbosch & 1964 & 1968 & 4 synonyms \\
\hline & Innsbruck & 2000 & 2003 & \\
\hline & Gent & 1965 & 1970 & $\begin{array}{l}\text { arrived as L. bromfieldii } \\
\text { var. insularis }\end{array}$ \\
\hline & Muenster & 1968 & 1971 & \\
\hline & Nantes & 1993 & 2000 & \\
\hline Lithops comptonii L. Bolus & Kirstenbosch & 1957 & 1961 & arrived as L. weberi \\
\hline Lithops dorotheae $\mathrm{Nel}$ & Nantes & 1992 & 1997 & syn. L. eksteeniae \\
\hline \multirow[t]{2}{*}{ Lithops gesineae de Boer } & Nantes & 1996 & 2000 & $\begin{array}{l}\text { arrived as L. annae; one of } \\
2 \text { synonyms }\end{array}$ \\
\hline & Gent & 1976 & 1982 & $\begin{array}{l}\text { arrived as } L . \text { francisci } \\
\text { var. annae }\end{array}$ \\
\hline Lithops gracilidelineata Dinter & Monaco & 1991 & 1997 & 2 synonyms \\
\hline Lithops hallii de Boer & Kirstenbosch & 1964 & 1968 & 2 synonyms \\
\hline \multirow[t]{6}{*}{ Lithops hookeri (A. Berger) Schwantes } & Kirstenbosch & 1957 & 1961 & $\begin{array}{l}\text { arrived as L. turbiniformis; } \\
20+\text { synonyms }\end{array}$ \\
\hline & Gent & 1979 & 1982 & \\
\hline & Nantes & 1993 & 1995 & \\
\hline & Dresden & 1993 & 2000 & \\
\hline & Gent & 1967 & 1971 & $\begin{array}{l}\text { arrived as L. turbiniformis } \\
\text { var. marginata }\end{array}$ \\
\hline & Nantes & 1999 & 2000 & \\
\hline Lithops julii (Dinter \& Schwantes) N.E. Br. & Gent & 1967 & 1971 & $\begin{array}{l}\text { arrived as L. julii } \\
\text { subsp. julii; one of } 2 \\
\text { synonyms }\end{array}$ \\
\hline \multirow[t]{2}{*}{ Lithops julii subsp. fulleri (N.E. Br.) B. Fearn } & Kirstenbosch & 1964 & 1971 & arrived as L. fulleri \\
\hline & Gent & 1967 & 1971 & $\begin{array}{l}\text { arrived as L. fulleri } \\
\text { v. fulleri }\end{array}$ \\
\hline \multirow[t]{3}{*}{$\begin{array}{l}\text { Lithops karasmontana (Dinter \& Schwantes) N.E. } \\
\text { Br. }\end{array}$} & Gent & 1967 & 1971 & $\begin{array}{l}\text { arrived as L. karasmontana } \\
\text { subsp. karasmontana; one } \\
\text { of } 20+\text { synonyms }\end{array}$ \\
\hline & Muenster & $1966 \& 1967$ & $1968 \& 1977$ & \\
\hline & Monaco & 1991 & 2000 & \\
\hline
\end{tabular}


S8 Sandev, D. et al.: Plethora of plants - collections of the Botanical Garden, Faculty of Science, University of Zagreb (2)

\begin{tabular}{|c|c|c|c|c|}
\hline Scientific name & $\begin{array}{l}\text { City of plant } \\
\text { material origin }\end{array}$ & $\begin{array}{l}\text { Year of } \\
\text { ordering/ } \\
\text { purchasing }\end{array}$ & $\begin{array}{l}\text { Year when plant } \\
\text { was last recorded } \\
\text { in collection }\end{array}$ & Notes and remarks \\
\hline & Gent & 1979 & 1980 & $\begin{array}{l}\text { arrived as L. karasmontana } \\
\text { var. mickbergensis }\end{array}$ \\
\hline & Muenster & 1968 & 1971 & arrived as L. lateritia \\
\hline & Antwerpen & unknown & 1961 & arrived as L. lericheana \\
\hline & Gent & $\begin{array}{l}1963 \& 1964 \\
\& 1965\end{array}$ & $\begin{array}{l}1971 \& 1968 \& \\
1971\end{array}$ & \\
\hline & Muenster & 1968 & 1971 & \\
\hline & Muenster & 1968 & 1971 & arrived as L. mickbergensis \\
\hline & Muenster & 1966 & 1968 & arrived as L. opalina \\
\hline \multirow[t]{3}{*}{$\begin{array}{l}\text { Lithops karasmontana subsp. eberlanzii (Dinter \& } \\
\text { Schwantes) D. T. Cole }\end{array}$} & Gent & 1979 & 1982 & $\begin{array}{l}\text { arrived as L. bella } \\
\text { var. eberlanzii; one of } 6 \\
\text { synonyms }\end{array}$ \\
\hline & Kirstenbosch & 1964 & 1968 & arrived as L. erniana \\
\hline & Chemnitz & 1995 & 2000 & \\
\hline \multirow[t]{2}{*}{ Lithops lesliei (N.E. Br.) N.E. Br. subsp. lesliei } & Gent & 1979 & 1983 & $\begin{array}{l}\text { arrived as L. lesliei } \\
\text { var. minor }\end{array}$ \\
\hline & Muenster & 1968 & 1977 & arrived as L. venteri \\
\hline \multirow[t]{6}{*}{ Lithops localis (N.E. Br.) Schwantes } & Pecs & 1984 & 1985 & 5 synonyms \\
\hline & Potsdam & 1985 & 1993 & \\
\hline & Basel & 1986 & 1990 & \\
\hline & Innsbruck & 2008 & 2014 & \\
\hline & Bordeaux & 1984 & 1990 & $\begin{array}{l}\text { arrived as L. localis } \\
\text { var. peersii }\end{array}$ \\
\hline & Monaco & 1999 & 2000 & arrived as L. terricolor \\
\hline \multirow[t]{3}{*}{ Lithops marmorata (N.E. Br.) N.E. Br. } & Kiel & 1968 & 1961 & $\begin{array}{l}\text { arrived as L. marmorata } \\
\text { var. elisae; one of } 5 \\
\text { synonyms }\end{array}$ \\
\hline & Muenster & $1967 \& 1968$ & $1971 \& 1971$ & \\
\hline & Nantes & 1999 & 2000 & \\
\hline Lithops 'Mezcla' & Barcelona & 1963 & 1968 & $\begin{array}{l}\text { unknown cultivar; esp. } \\
\text { mezcla - eng. mixing }\end{array}$ \\
\hline Lithops olivacea L. Bolus & Chemnitz & 1993 & $1993-2000$ & $\begin{array}{l}\text { syn. L. olivacea } \\
\text { var. nebrownii }\end{array}$ \\
\hline Lithops optica (Marloth) N.E. Br. & Gent & 1967 & 1971 & $\begin{array}{l}\text { arrived as L. elevata; one of } \\
2 \text { synonyms }\end{array}$ \\
\hline Lithops otzeniana $\mathrm{Nel}$ & Dresden & 1996 & 2000 & \\
\hline \multirow{9}{*}{ Lithops pseudotruncatella (A. Berger) N.E. Br. } & Kiel & 1955 & no data & $15+$ synonyms \\
\hline & Kirstenbosch & 1957 & 1975 & \\
\hline & Gent & $1964 \& 1965$ & $1968 \& 1968$ & \\
\hline & Berlin & 1964 & 1968 & \\
\hline & Pecs & 1984 & no data & \\
\hline & Monaco & 1991 & 2000 & \\
\hline & Kiel & $1955 \& 1958$ & no data \& 1963 & arrived as L. alpina \\
\hline & Muenster & 1968 & 1971 & \\
\hline & Gent & 1976 & 1982 & $\begin{array}{l}\text { arrived as L. pseudotrunca- } \\
\text { tella var. alpina }\end{array}$ \\
\hline \multirow[t]{2}{*}{$\begin{array}{l}\text { Lithops pseudotruncatella subsp. dendritica (Nel) } \\
\text { D. T. Cole }\end{array}$} & Kiel & 1958 & 1963 & $\begin{array}{l}\text { arrived as L. dendritica; } \\
\text { one of } 2 \text { synonyms }\end{array}$ \\
\hline & Gent & $1963 \& 1964$ & $1968 \& 1968$ & \\
\hline \multirow[t]{3}{*}{ Lithops ruschiorum (Dinter \& Schwantes) N.E. Br. } & Groningen & 1962 & 1966 & $\begin{array}{l}\text { syn. Mesembryanthemum } \\
\text { ruschiorum }\end{array}$ \\
\hline & Gent & 1979 & 1982 & \\
\hline & Dresden & 1996 & 1997 & \\
\hline \multirow[t]{8}{*}{ Lithops salicola L. Bolus } & Berlin & 1958 & 1968 & \\
\hline & Tuebingen & $1963 \& 1973$ & $1971 \& 1978$ & \\
\hline & Marburg & 1962 & 1970 & \\
\hline & Muenster & $1961 \& 1967$ & $1977 \& 1978$ & \\
\hline & Vienna & 1971 & no data & \\
\hline & Potsdam & 1985 & no data & \\
\hline & Dronten & 1984 & no data & \\
\hline & Nantes & 2000 & 2016 & \\
\hline \multirow[t]{2}{*}{ Lithops schwantesii Dinter } & Gent & \begin{tabular}{|l|}
$1963 \& 1964 \& \&$ \\
$1965 \& 1966$
\end{tabular} & $\begin{array}{l}1977 \& 1968 \& \\
1968 \& 1968\end{array}$ & $10+$ synonyms \\
\hline & Pecs & 1984 & 2000 & \\
\hline
\end{tabular}




\begin{tabular}{|c|c|c|c|c|}
\hline Scientific name & $\begin{array}{l}\text { City of plant } \\
\text { material origin }\end{array}$ & $\begin{array}{l}\text { Year of } \\
\text { ordering/ } \\
\text { purchasing }\end{array}$ & $\begin{array}{l}\text { Year when plant } \\
\text { was last recorded } \\
\text { in collection }\end{array}$ & Notes and remarks \\
\hline & Monaco & 1996 & 1997 & \\
\hline & Chemnitz & 1997 & 2000 & \\
\hline & Nantes & $1999 \& 2000$ & $2001 \& 2001$ & \\
\hline & Muenster & $1966 \& 1967$ & $1970 \& 1971$ & arrived as L. kuibisensis \\
\hline & Gent & $1966 \& 1967$ & $1970 \& 1971$ & \\
\hline & Nantes & 1996 & 1997 & arrived as L. marthae \\
\hline & Gent & 1962 & 1968 & arrived as L. rugosa \\
\hline & Muenster & 1968 & 1971 & \\
\hline & Basel & 1984 & 1985 & \\
\hline & Muenster & 1967 & 1971 & arrived as L. triebneri \\
\hline & Gent & 1965 & 1968 & arrived as L. kunjasensis \\
\hline & Graz & 1991 & 2000 & \\
\hline Lithops vallis-mariae (Dinter \& Schwantes) N.E. Br. & Kiel & 1958 & 1963 & $\begin{array}{l}\text { syn. Mesembryanthemum } \\
\text { vallis-mariae }\end{array}$ \\
\hline Lithops verruculosa $\mathrm{Nel}$ & Nantes & 1996 & 2000 & $\begin{array}{l}\text { arrived as L. inae; one of } 3 \\
\text { synonyms }\end{array}$ \\
\hline \multirow[t]{4}{*}{ Lithops villetii L. Bolus } & Kiel & 1955 & 1961 & $\begin{array}{l}\text { syn. L. villetii subsp. vil- } \\
\text { letii }\end{array}$ \\
\hline & Kirstenbosch & 1961 & 1962 & \\
\hline & Gent & $1970 \& 1976$ & $1977 \& 1983$ & \\
\hline & Dresden & 1986 & no data & \\
\hline Lithops werneri Schwantes \& H. Jacobsen & Muenchen & 1993 & 1997 & \\
\hline \multirow[t]{3}{*}{ Machairophyllum albidum (L.) Schwantes } & Gent & $\begin{array}{l}1960 \& 1961 \\
\& 1962\end{array}$ & $\begin{array}{l}1968 \& 2010 \& \\
1979\end{array}$ & 3 synonyms \\
\hline & Vienna & 1971 & 2010 & \\
\hline & Pecs & 1996 & 2003 & \\
\hline Mesembryanthemum aitonis Jacq. & Muenchen & 1978 & 1980 & 5 synonyms \\
\hline Mesembryanthemum vanrensburgii (L. Bolus) Klak & Barcelona & unknown & 1961 & $\begin{array}{l}\text { arrived as Prenia } \\
\text { vanrensburgii; one of } 2 \\
\text { synonyms }\end{array}$ \\
\hline \multirow[t]{2}{*}{ Mestoklema tuberosum (L.) N.E. Br. ex Glen } & Kiel & 1998 & 2003 & 4 synonyms \\
\hline & nn & $\begin{array}{l}\text { in collection } \\
\text { in } 1963\end{array}$ & 1975 & \\
\hline Mitrophyllum clivorum Schwantes & Kirstenbosch & 1961 & 1968 & \\
\hline Monilaria moniliformis Ihlenf. \& Joergens. & Hamburg & 1976 & 1979 & \\
\hline $\begin{array}{l}\text { Monilaria scutata Schwantes subsp. obovata Ihlenf. } \\
\text { \& Joergens. }\end{array}$ & Hamburg & 1976 & 1979 & \\
\hline Nananthus aloides (Haw.) Schwantes & Cantonspark & 1955 & 1961 & 4 synonyms \\
\hline Nananthus vittatus (N.E. Br.) Schwantes & Kiel & 1958 & 1961 & 4 synonyms \\
\hline \multirow[t]{5}{*}{ Orthopterum waltoniae L. Bolus } & Barcelona & $1958 \& 1963$ & $1968 \& 1968$ & \\
\hline & Gent & 1970 & 1981 & \\
\hline & Berlin & $1972 \& 1973$ & $1981 \& 1981$ & \\
\hline & Innsbruck & 1997 & 2000 & \\
\hline & Muenchen & 2000 & 2002 & \\
\hline Oscularia caulescens (Mill.) Schwantes & Bochum & 1998 & 2003 & 3 synonyms \\
\hline \multirow[t]{2}{*}{ Oscularia deltoides (L.) Schwantes } & nn & 1961 & 1971 & 2 synonyms \\
\hline & Berlin & $1967 \& 1974$ & $1968 \& 1980$ & \\
\hline Oscularia lunata (Willd.) H. E. K. Hartmann & Berlin & 1979 & 2000 & $\begin{array}{l}\text { arrived as Lampranthus } \\
\text { lunatus; one of } 2 \\
\text { synonyms }\end{array}$ \\
\hline Peersia frithii (L. Bolus) L. Bolus & Gent & 1964 & 1968 & $\begin{array}{l}\text { arrived as Rhinephyllum } \\
\text { frithii; one of } 2 \text { synonyms }\end{array}$ \\
\hline Phyllobolus prasinus (L.Bolus) Gerbaulet & Muenchen & 1970 & 1972 & arrived as Aridaria prasina \\
\hline \multirow[t]{3}{*}{ Phyllobolus splendens (L.) Gerbaulet } & nn & unknown & 1961 & $\begin{array}{l}\text { arrived as Aridaria } \\
\text { flexuosa; one of } 20+ \\
\text { synonyms }\end{array}$ \\
\hline & Kirstenbosch & 1961 & 1961 & $\begin{array}{l}\text { arrived as Aridaria } \\
\text { primulina }\end{array}$ \\
\hline & nn & unknown & 1963 & $\begin{array}{l}\text { arrived as Aridaria } \\
\text { splendens }\end{array}$ \\
\hline \multirow[t]{4}{*}{ Pleiospilos bolusii (Hook. f.) N.E. Br. } & Vienna & 1955 & unknown & \\
\hline & Berlin & $1955 \& 1961$ & $\begin{array}{l}\text { unknown \& } \\
1961\end{array}$ & \\
\hline & Lisbon & 1955 & unknown & \\
\hline & Palermo & $1961 \& 1964$ & $1971 \& 1968$ & \\
\hline
\end{tabular}


S10 Sandev, D. et al.: Plethora of plants - collections of the Botanical Garden, Faculty of Science, University of Zagreb (2)

\begin{tabular}{|c|c|c|c|c|}
\hline Scientific name & $\begin{array}{l}\text { City of plant } \\
\text { material origin }\end{array}$ & $\begin{array}{l}\text { Year of } \\
\text { ordering/ } \\
\text { purchasing }\end{array}$ & $\begin{array}{l}\text { Year when plant } \\
\text { was last recorded } \\
\text { in collection }\end{array}$ & Notes and remarks \\
\hline & Barcelona & 1964 & 1968 & \\
\hline & Muenchen & 1967 & unknown & \\
\hline & Karlsruhe & 1969 & 1977 & \\
\hline & Nantes & 1970 & 1977 & \\
\hline & Wuppertal & 1985 & 1989 & \\
\hline \multirow[t]{2}{*}{ Pleiospilos compactus (Aiton) Schwantes } & nn & 1952 & 1975 & $\begin{array}{l}\text { arrived as } P \text {. longibractea- } \\
\text { tus; one of } 7 \text { synonyms }\end{array}$ \\
\hline & Palermo & 1964 & 1971 & \\
\hline \multirow[t]{24}{*}{$\begin{array}{l}\text { Pleiospilos compactus subsp. canus (Haw.) H. E. K. } \\
\text { Hartmann \& Liede }\end{array}$} & Berlin & $1962 \& 1965$ & $1969 \& 1971$ & $\begin{array}{l}\text { arrived as } P \text {. borealis; one } \\
\text { of } 20+\text { synonyms }\end{array}$ \\
\hline & nn & 1951 & 1983 & arrived as $P$. brevisepalus \\
\hline & Berlin & $1962 \& 1967$ & $1968 \& 1968$ & \\
\hline & Muenster & 1967 & 1968 & \\
\hline & Gent & $1962 \& 1964$ & $1975 \& 1968$ & arrived as P. dekenahi \\
\hline & Palermo & 1964 & 1968 & \\
\hline & Berlin & 1962 & 1971 & arrived as P.framesii \\
\hline & nn & 1951 & 1963 & arrived as $P$. grandiflorus \\
\hline & Palermo & 1964 & 1971 & \\
\hline & Frankfurt & unknown & 1961 & arrived as $P$. latipetalus \\
\hline & Nantes & 1996 & 1997 & arrived as P. leipoldtii \\
\hline & nn & 1951 & 1961 & $\begin{array}{l}\text { arrived as P. magnipuncta- } \\
\text { tus }\end{array}$ \\
\hline & Palermo & 1961 & 1961 & \\
\hline & Kirstenbosch & 1971 & 1978 & \\
\hline & Pretoria & 1975 & 1980 & \\
\hline & Berlin & 1955 & 1961 & arrived as P. nobilis \\
\hline & Innsbruck & 2000 & 2002 & \\
\hline & nn & 1951 & no data & arrived as P. peersii \\
\hline & Berlin & 1961 & 1961 & \\
\hline & Palermo & 1964 & 1966 & \\
\hline & Gent & $1962 \& 1964$ & $1968 \& 1968$ & $\begin{array}{l}\text { arrived as P. willowmoren- } \\
\text { sis }\end{array}$ \\
\hline & Basel & 1962 & 1968 & \\
\hline & Kirstenbosch & 1964 & 1971 & \\
\hline & Berlin & 1967 & 1968 & \\
\hline \multirow[t]{4}{*}{$\begin{array}{l}\text { Pleiospilos compactus subsp. sororius H.E.K. } \\
\text { Hartmann \& Liede }\end{array}$} & Berlin & $1962 \& 1967$ & $1971 \& 1973$ & $\begin{array}{l}\text { arrived as } P \text {. dimidiatus; } \\
\text { one of } 2 \text { synonyms }\end{array}$ \\
\hline & nn & unknown & 1968 & \\
\hline & Muenchen & $1993 \& 1994$ & $2000 \& 1997$ & \\
\hline & Graz & 1994 & 2000 & \\
\hline Pleiospilos nelii Schwantes & Kirstenbosch & 1961 & 1968 & 2 synonyms \\
\hline Prenia pallens (Aiton) N.E. Br. subsp. pallens & $\mathrm{nn}$ & 1950 & no data & arrived as $P$. relaxata \\
\hline Prepodesma orpenii (N.E. Br.) N.E. Br. & Barcelona & 1963 & 1968 & \begin{tabular}{|l|} 
arrived as Nananthus \\
orpenii; one of 5 synonyms
\end{tabular} \\
\hline Psammophora nissenii (Dinter) Dinter \& Schwantes & Kiel & 1958 & 1961 & $\begin{array}{l}\text { syn. Mesembryanthemum } \\
\text { nissenii }\end{array}$ \\
\hline Psilocaulon dinteri Schwantes & Berlin & 1964 & 1971 & $10+$ synonyms \\
\hline Psilocaulon subnodosum (A. Berger) N.E. Br. & Barcelona & 1963 & 1966 & $\begin{array}{l}\text { arrived as P.clavulatum; } \\
\text { one of } 6 \text { synonyms }\end{array}$ \\
\hline Rabiea albinota (Haw.) N.E. Br. & Barcelona & 1969 & 1971 & 3 synonyms \\
\hline \multirow[t]{3}{*}{ Rabiea cibdela (N.E.Br.) N.E.Br. } & Vienna & $1955 \& 1965$ & $1989 \& 1981$ & $\begin{array}{l}\text { syn. Mesembryanthemum } \\
\text { cibdelum }\end{array}$ \\
\hline & Haren & 1970 & 1978 & \\
\hline & Pecs & 1996 & 1997 & \\
\hline Rabiea difformis (L. Bolus) L. Bolus & Bochum & 2003 & 2010 & syn. Nananthus difformis \\
\hline Rhinephyllum broomii L. Bolus & Berlin & 1965 & no data & \\
\hline Rhinephyllum comptonii L. Bolus & Berlin & 1971 & 1983 & \\
\hline Rhinephyllum graniforme (Haw.) L. Bolus & Berlin & 1971 & 1979 & $\begin{array}{l}\text { syn. Mesembryanthemum } \\
\text { graniforme }\end{array}$ \\
\hline \multirow{2}{*}{$\begin{array}{l}\text { Rhombophyllum dyeri (L. Bolus) H. E. K. } \\
\text { Hartmann }\end{array}$} & Muenchen & 1966 & 1971 & arrived as Hereroa dyeri \\
\hline & Berlin & $1971 \& 1974$ & 1980 & \\
\hline
\end{tabular}




\begin{tabular}{|c|c|c|c|c|}
\hline Scientific name & $\begin{array}{l}\text { City of plant } \\
\text { material origin }\end{array}$ & $\begin{array}{l}\text { Year of } \\
\text { ordering/ } \\
\text { purchasing }\end{array}$ & $\begin{array}{l}\text { Year when plant } \\
\text { was last recorded } \\
\text { in collection }\end{array}$ & Notes and remarks \\
\hline \multirow[t]{3}{*}{ Rhombophyllum nelii Schwantes } & Berlin & $\begin{array}{l}1971 \& 1973 \\
\& 1978\end{array}$ & $\begin{array}{l}1978 \& 1977 \& \\
1980\end{array}$ & \\
\hline & Muenchen & $1970 \& 1971$ & $1974 \& 1975$ & \\
\hline & Gent & $1976 \& 1979$ & $1980 \& 1989$ & \\
\hline Ruschia cymosa (L. Bolus) Schwantes & Kiel & 1958 & 1961 & $\begin{array}{l}\text { syn. Mesembryanthemum } \\
\text { cymosum }\end{array}$ \\
\hline Ruschia hexamera L. Bolus & Berlin & 1971 & 1979 & 2 synonyms \\
\hline Ruschia multiflora (Haw.) Schwantes & Berlin & $1971 \& 1973$ & $1979 \& 1982$ & $\begin{array}{l}\text { syn. Mesembryanthemum } \\
\text { multiflorum }\end{array}$ \\
\hline \multirow[t]{2}{*}{ Ruschia perfoliata (Mill.) Schwantes } & Barcelona & 1968 & 1979 & $\begin{array}{l}\text { syn. Mesembryanthemum } \\
\text { perfoliatum }\end{array}$ \\
\hline & Muenchen & 1970 & 1978 & \\
\hline \multirow[t]{2}{*}{ Ruschia rigida (Haw.) Schwantes } & Lisbon & 1954 & 1961 & $\begin{array}{l}\text { syn. Mesembryanthemum } \\
\text { rigidum }\end{array}$ \\
\hline & Berlin & 1966 & 1968 & \\
\hline Ruschia rubricaulis (Haw.) L. Bolus & Kirstenbosch & 1988 & 1990 & $\begin{array}{l}\text { syn. Mesembryanthemum } \\
\text { rubricaule }\end{array}$ \\
\hline Ruschia vaginata Schwantes & Berlin & 1973 & 2010 & $\begin{array}{l}\text { syn. Mesembryanthemum } \\
\text { vaginatum }\end{array}$ \\
\hline Semnanthe lacera (Haw.) N.E. Br. & Berlin & 1955 & & 4 synonyms \\
\hline Stomatium bolusiae Schwantes & Nantes & 1996 \& 1998 & 1997 \& no data & \\
\hline Stomatium duthiae L. Bolus & Nantes & 1998 & 2000 & \\
\hline \multirow[t]{2}{*}{$\begin{array}{l}\text { Tanquana archeri (L. Bolus) H.E.K. Hartmann \& } \\
\text { Liede }\end{array}$} & Berlin & 1967 & 1971 & $\begin{array}{l}\text { arrived as Pleiospilos } \\
\text { archeri; one of } 7 \text { synonyms }\end{array}$ \\
\hline & Nantes & $1970 \& 1996$ & $1975 \& 1997$ & \\
\hline $\begin{array}{l}\text { Tanquana hilmarii (L. Bolus) H. E. K. Hartmann } \\
\text { \& Liede }\end{array}$ & Berlin & 1955 & 1961 & $\begin{array}{l}\text { arrived as Pleiospilos } \\
\text { hilmarii }\end{array}$ \\
\hline \multirow[t]{2}{*}{ Titanopsis calcarea (Marloth) Schwantes } & Gent & 1963 & 1966 & 2 synonyms \\
\hline & Kiel & 1997 & 1998 & \\
\hline \multirow[t]{2}{*}{ Trichodiadema barbatum Schwantes } & Muenchen & 1970 & no data & \\
\hline & Berlin & $\begin{array}{l}1973 \& 1978 \\
\& 1979 \& \\
2002 \& 2003 \\
\end{array}$ & $\begin{array}{l}1979 \& 1990 \& \\
2000 \& 2003 \& \\
2003\end{array}$ & \\
\hline \multirow[t]{2}{*}{ Trichodiadema bulbosum Schwantes } & nn & 1951 & 1966 & \\
\hline & Vienna & 1956 & no data & \\
\hline \multirow[t]{2}{*}{ Trichodiadema densum Schwantes } & Beograd & 1965 & 1968 & \\
\hline & nn & unknown & 1968 & \\
\hline \multirow[t]{2}{*}{ Trichodiadema intonsum Schwantes } & nn & 1952 & 1961 & \\
\hline & Gent & 1966 & 1968 & \\
\hline \multirow[t]{4}{*}{ Trichodiadema mirabile (N.E. Br.) Schwantes } & Muenchen & 1970 & 1978 & 2 synonyms \\
\hline & Szeged & 1970 & 1978 & \\
\hline & Berlin & 1979 & 2000 & \\
\hline & Nantes & 2000 & 2001 & \\
\hline Trichodiadema peersii L. Bolus & Gent & 1970 & 1979 & \\
\hline Trichodiadema rogersiae L. Bolus & Berlin & 1971 & 1980 & \\
\hline Trichodiadema setuliferum Schwantes & Berlin & 1971 & 1978 & \\
\hline $\begin{array}{l}\text { Vanheerdea roodiae (N.E. Br.) L. Bolus ex H. E. K. } \\
\text { Hartmann }\end{array}$ & Kirstenbosch & 1961 & 1968 & $\begin{array}{l}\text { arrived as } V \text {. divergens; one } \\
\text { of } 5 \text { synonyms }\end{array}$ \\
\hline \multicolumn{5}{|l|}{ Apocynaceae } \\
\hline Adenium obesum (Forssk.) Roem. \& Schult. & Muenchen & 1997 & 2000 & $10+$ synonyms \\
\hline Pachypodium lamerei Drake & Monaco & 1993 & 2000 & 6 synonyms \\
\hline Plumeria rubra L. & Bogor & 1982 & 1982 & $20+$ synonyms \\
\hline \multicolumn{5}{|l|}{ Asclepiadaceae } \\
\hline \multirow[t]{2}{*}{ Caralluma burchardii N.E.Br. var. maura Maire } & Antwerpen & 1957 & 1998 & 2 synonyms \\
\hline & nn & $\begin{array}{l}\text { in collection } \\
\text { in } 1975\end{array}$ & 1980 & \\
\hline \multirow[t]{4}{*}{ Caralluma europaea (Guss.) N.E.Br. } & Basel & 1956 & 1983 & $30+$ synonyms \\
\hline & Gent & 1962 & 1972 & \\
\hline & Bologna & 1971 & 1979 & \\
\hline & Lyon & 1998 & 2000 & \\
\hline Caralluma gracilipes K.Schum. & Goettingen & 1970 & 1979 & $\begin{array}{l}\text { syn. Spathulopetalum } \\
\text { gracilipes }\end{array}$ \\
\hline
\end{tabular}


S12 Sandev, D. et al.: Plethora of plants - collections of the Botanical Garden, Faculty of Science, University of Zagreb (2)

\begin{tabular}{|c|c|c|c|c|}
\hline Scientific name & $\begin{array}{l}\text { City of plant } \\
\text { material origin }\end{array}$ & $\begin{array}{l}\text { Year of } \\
\text { ordering/ } \\
\text { purchasing }\end{array}$ & $\begin{array}{l}\text { Year when plant } \\
\text { was last recorded } \\
\text { in collection }\end{array}$ & Notes and remarks \\
\hline Caralluma nebrownii A. Berger & Palermo & 1964 & 1966 & $\begin{array}{l}\text { syn. Orbeopsis lutea subsp. } \\
\text { vaga; unresolved name } \\
\text { (The Plant List) }\end{array}$ \\
\hline \multirow[t]{2}{*}{ Caralluma subulata (Forssk.) Decne. } & nn & $\begin{array}{l}\text { in collection } \\
\text { in } 1965\end{array}$ & 1972 & syn. Stapelia subulata \\
\hline & Goettingen & 1970 & 1979 & \\
\hline \multirow[t]{5}{*}{ Ceropegia africana R.Br. } & Liberec & 1961 & 1972 & \\
\hline & Vacratot & $\begin{array}{l}\text { unknown \& } \\
1969\end{array}$ & $1961 \& 1972$ & \\
\hline & Gent & 1962 & 1963 & \\
\hline & Strasboug & $1966 \& 1969$ & $1980 \& 1984$ & \\
\hline & Goeteborg & 1970 & 1972 & \\
\hline \multirow[t]{4}{*}{$\begin{array}{l}\text { Ceropegia albisepta Jum. \& H.Perrier var. } \\
\text { robynsiana (Werderm.) H.Huber }\end{array}$} & Antwerpen & $\begin{array}{l}1956 \& 1958 \\
\& 1962\end{array}$ & $\begin{array}{l}1961 \& 1966 \& \text { \& } \\
1981\end{array}$ & arrived as C. robynsiana \\
\hline & Besancon & 1959 & 1979 & \\
\hline & nn & $\begin{array}{l}\text { in collection } \\
\text { in } 1963\end{array}$ & 1981 & \\
\hline & Strasburg & 1969 & 1980 & \\
\hline Ceropegia ballyana Bullock & Karlsruhe & 1969 & 1981 & syn. C. helicoides \\
\hline \multirow[t]{2}{*}{ Ceropegia bulbosa Roxb. } & nn & $\begin{array}{l}\text { in collection } \\
\text { in } 1963\end{array}$ & 1981 & from cuttings; 9 synonyms \\
\hline & Besancon & 1968 & 1988 & from cuttings \\
\hline Ceropegia dichotoma Haw. & Litva & 1957 & 1961 & syn. C. aphylla \\
\hline \multirow[t]{2}{*}{ Ceropegia distincta N.E.Br. } & Besancon & $1969 \& 1970$ & $1972 \& 1979$ & syn. C. brevirostris \\
\hline & Tuebingen & 1971 & 1981 & \\
\hline $\begin{array}{l}\text { Ceropegia distincta subsp. haygarthii (Schltr.) H. } \\
\text { Huber }\end{array}$ & Debrecen & 1980 & 1984 & syn. C. haygarthii \\
\hline Ceropegia gemmifera K.Schum. & Legon & 1970 & 1972 & syn. C. nilotica \\
\hline \multirow[t]{4}{*}{ Ceropegia haygarthii Schltr. } & Antwerpen & $1969 \& 1972$ & $1979 \& 1984$ & $\begin{array}{l}\text { syn. C. distincta subsp. } \\
\text { haygarthii }\end{array}$ \\
\hline & nn & 1970 & 1972 & \\
\hline & nn & 1981 & 1972 & \\
\hline & Bergen & 1972 & 1972 & \\
\hline $\begin{array}{l}\text { Ceropegia linearis E.Mey. subsp. debilis (N.E.Br.) } \\
\text { H.Huber }\end{array}$ & Krakow & 1970 & 1972 & arrived as syn. C. debilis \\
\hline Ceropegia multiflora Baker & Goeteborg & 1977 & 1980 & $\begin{array}{l}\text { syn. C. multiflora subsp. } \\
\text { multiflora }\end{array}$ \\
\hline \multirow[t]{4}{*}{ Ceropegia sandersonii Decne. ex Hook.f. } & nn & $\begin{array}{l}1951 \& \\
\text { unknown }\end{array}$ & $1978 \& 1968$ & $\begin{array}{l}2 \text { synonyms; from cuttings } \\
\text { (unknown) }\end{array}$ \\
\hline & Strasbourg & 1969 & 1980 & \\
\hline & Warsaw & 1986 & 1988 & from cuttings \\
\hline & Salaspils & 2000 & 2003 & \\
\hline \multirow[t]{3}{*}{ Ceropegia stapeliiformis Haw. } & nn & $\begin{array}{l}1951 \& \text { in } \\
\text { collection in } \\
1968\end{array}$ & no data \& 1978 & $\begin{array}{l}\text { syn. C. stapeliiformis subsp. } \\
\text { stapeliiformis; from } \\
\text { cuttings (1951) }\end{array}$ \\
\hline & Cantonspark & 1953 & 1968 & \\
\hline & Strasbourg & 1969 & 1978 & \\
\hline \multirow[t]{7}{*}{ Ceropegia woodii Schltr. } & Cluj-Napoca & 1958 & 1961 & $\begin{array}{l}\text { arrived as C. hastata; one } \\
\text { of } 7 \text { synonyms }\end{array}$ \\
\hline & Strasboug & 1969 & 1972 & \\
\hline & Barcelona & 1970 & 1972 & \\
\hline & Kaunas & 1970 & 1979 & \\
\hline & Goeteborg & 1970 & 1982 & \\
\hline & nn & $\begin{array}{l}\text { in collection } \\
\text { in } 1963 \text { \& in } \\
\text { collection in } \\
1979\end{array}$ & 1978 \& 2000 & from cuttings (1963) \\
\hline & Krakow & 1970 & 1982 & \\
\hline \multirow[t]{3}{*}{ Cynanchum viminale (L.) L. } & Stockholm & 1955 & 2000 & $\begin{array}{l}\text { from cuttings; arrived as } \\
\text { Sarcostemma viminale; one } \\
\text { of } 10+\text { synonyms }\end{array}$ \\
\hline & Antwerpen & 1958 & & \\
\hline & Nantes & 1996 & 1997 & \\
\hline Dischidia bengalensis Colebr. & nn & $\begin{array}{l}\text { in collection } \\
\text { in } 1963\end{array}$ & 1972 & \\
\hline
\end{tabular}




\begin{tabular}{|c|c|c|c|c|}
\hline Scientific name & $\begin{array}{l}\text { City of plant } \\
\text { material origin }\end{array}$ & $\begin{array}{l}\text { Year of } \\
\text { ordering/ } \\
\text { purchasing }\end{array}$ & $\begin{array}{l}\text { Year when plant } \\
\text { was last recorded } \\
\text { in collection }\end{array}$ & Notes and remarks \\
\hline Dischidia hirsuta (Blume) Decne. & Goeteborg & 1973 & 1975 & syn. Leptostemma hirsutum \\
\hline Dischidia longinense & Berlin & 1960 & 1961 & unknown species \\
\hline Dischidia major (Vahl) Merr. & Bergen & $1965 \& 1966$ & $1966 \& 1981$ & arrived as D. rafflesiana \\
\hline Dischidia purpurea Merr. & Tuebingen & 1970 & 1972 & \\
\hline \multirow[t]{2}{*}{ Echidnopsis cereiformis Hook.f. } & nn & unknown & 1961 & \\
\hline & nn & 1960 & 1968 & \\
\hline $\begin{array}{l}\text { Echidnopsis cereiformis var. brunnea (Dammann) } \\
\text { A. Berger }\end{array}$ & Besancon & 1970 & 1975 & \\
\hline Echidnopsis dammanniana Sprenger & Palermo & 1964 & 1966 & syn. E. somalensis \\
\hline Hoodia gordonii (Masson) Sweet ex Decne. & Muenchen & 1976 & 1978 & syn. Stapelia gordonii \\
\hline \multirow{2}{*}{ Huernia erinacea P.R.O. Bally } & Liege & 1985 & 1985 & \\
\hline & Dresden & 1995 & 2000 & \\
\hline Huernia hystrix (Hook. f.) N.E. Br. & Stockholm & 1996 & 2000 & 3 synonyms \\
\hline \multirow[t]{3}{*}{ Huernia keniensis R. E. Fr. } & Basel & 1955 & no data & $\begin{array}{l}\text { syn. H. keniensis } \\
\text { var. keniensis }\end{array}$ \\
\hline & Cantonspark & 1955 & 1961 & \\
\hline & Rome & 1957 & 1966 & \\
\hline \multirow[t]{4}{*}{$\begin{array}{l}\text { Huernia macrocarpa (A. Rich.) Spreng. } \\
\text { f. macrocarpa }\end{array}$} & Antwerpen & 1970 & 1971 & $\begin{array}{l}\text { arrived as H. macrocarpa } \\
\text { var. penzigii; one of } 10 \\
\text { synonyms }\end{array}$ \\
\hline & Nancy & 1971 & no data & \\
\hline & Aachen & 1995 & 2016 & \\
\hline & Szeged & 1955 & 1961 & $\begin{array}{l}\text { arrived as H. penzigii; } \\
\text { from cuttings }\end{array}$ \\
\hline $\begin{array}{l}\text { Huernia macrocarpa var. schweinfurthii (A. Berger) } \\
\text { A.C. White \& B. Sloane }\end{array}$ & Cantonspark & 1953 & no data & $\begin{array}{l}\text { syn. H. penzigii var. } \\
\text { schweinfurthii }\end{array}$ \\
\hline \multirow[t]{2}{*}{ Huernia pendula E. A. Bruce } & Goettingen & 1966 & 1966 & \\
\hline & Goeteborg & 1978 & 1978 & \\
\hline Huernia schneideriana A. Berger & Rome & 1970 & 1971 & \\
\hline Luckhoffia beukmanii White \& Sloane & Dresden & 1996 & 2000 & $\begin{array}{l}\text { hybrid between Hoodia } \\
\text { gordonii } \times \text { Stapelia arenosa }\end{array}$ \\
\hline \multirow[t]{2}{*}{ Orbea longidens (N.E. Br.) L. C. Leach } & Giessen & 1961 & 1968 & $\begin{array}{l}\text { arrived as Stapelia } \\
\text { longidens }\end{array}$ \\
\hline & Bochum & 1989 & 2000 & \\
\hline \multirow[t]{11}{*}{ Orbea variegata (L.) Haw. } & nn & 1951 & no data & $\begin{array}{l}\text { arrived as Stapelia lepida; } \\
\text { one of } 20+\text { synonyms }\end{array}$ \\
\hline & Szeged & 1954 & no data & from cuttings \\
\hline & Cantonspark & 1955 & no data & from cuttings \\
\hline & Iasi & 1958 & 1968 & \\
\hline & Duisburg & $1989 \& 1995$ & $2000 \& 2000$ & \\
\hline & nn & unknown & 1961 & arrived as Stapelia variegata \\
\hline & Besancon & 1968 & 1968 & \\
\hline & Vacratot & 1969 & 1971 & \\
\hline & Budapest & 1969 & 1974 & \\
\hline & Padova & 1983 & 2003 & \\
\hline & Duisburg & 1995 & 2000 & $\begin{array}{l}\text { arrived as Stapelia variegata } \\
\text { L. var. atropurpurea }\end{array}$ \\
\hline \multirow[t]{2}{*}{ Orbea variegata (L.) Haw. (var. picta) } & Leipzig & 1960 & 1961 & $\begin{array}{l}\text { arrived as Stapelia variegata } \\
\text { (var. picta) }\end{array}$ \\
\hline & Palermo & 1958 & 1968 & \\
\hline \multirow[t]{2}{*}{ Orbea variegata (L.) Haw. (var. atrata) } & Vacratot & 1969 & 1971 & $\begin{array}{l}\text { Stapelia variegata (var. } \\
\text { atrata) }\end{array}$ \\
\hline & Duisburg & $1989 \& 1995$ & $1997 \& 2000$ & \\
\hline Orbea variegata (L.) Haw. (var. curtisii) & Madrid & 1996 & 1997 & $\begin{array}{l}\text { arrived as Stapelia variegata } \\
\text { (var.curtisii) }\end{array}$ \\
\hline \multirow[t]{3}{*}{ Orbea variegata (L.) Haw. (var. marmorata) } & Leipzig & 1960 & 1961 & $\begin{array}{l}\text { arrived as Stapelia variegata } \\
\text { (var.marmorata) }\end{array}$ \\
\hline & Vacratot & 1969 & 1990 & \\
\hline & Gent & 1979 & 1993 & \\
\hline Orbea variegata (L.) Haw. (var. planiflora) & Palermo & $\begin{array}{l}\text { in collection } \\
\text { in } 1968\end{array}$ & 1971 & $\begin{array}{l}\text { arrived as Stapelia variegata } \\
\text { (var. planiflora) }\end{array}$ \\
\hline Orbeopsis lutea (N.E. Br.) L.C. Leach subsp. lutea & Pecs & 1996 & 2003 & $\begin{array}{l}\text { arrived as Caralluma } \\
\text { nebrownii; } 3 \text { synonyms }\end{array}$ \\
\hline
\end{tabular}


S14 Sandev, D. et al.: Plethora of plants - collections of the Botanical Garden, Faculty of Science, University of Zagreb (2)

\begin{tabular}{|c|c|c|c|c|}
\hline Scientific name & $\begin{array}{l}\text { City of plant } \\
\text { material origin }\end{array}$ & $\begin{array}{l}\text { Year of } \\
\text { ordering/ } \\
\text { purchasing }\end{array}$ & $\begin{array}{l}\text { Year when plant } \\
\text { was last recorded } \\
\text { in collection }\end{array}$ & Notes and remarks \\
\hline Philibertia gilliesii Hook. \& Arn. & Besancon & $1962 \& 1965$ & $1963 \& 1968$ & $\begin{array}{l}\text { arrived as } P \text {. gracilis; one } \\
\text { of } 4 \text { synonyms }\end{array}$ \\
\hline \multirow[t]{2}{*}{$\begin{array}{l}\text { Piaranthus geminatus (Masson) N.E. Br. } \\
\text { subsp. geminatus }\end{array}$} & Goeteborg & 1978 & 1978 & $\begin{array}{l}\text { arrived as } P \text {. foetidus; one } \\
\text { opf } 4 \text { synonyms }\end{array}$ \\
\hline & nn & 1969 & 1981 & \\
\hline Stapelia angulata Tod. & Antwerpen & 1965 & 1968 & \\
\hline \multirow{2}{*}{ Stapelia bella A. Berger } & Muenster & 1968 & 1983 & \\
\hline & Duisburg & 1989 & 2000 & \\
\hline \multirow[t]{2}{*}{ Stapelia discolor Tod. } & nn & 1952 & 1966 & \\
\hline & Vacratot & 1969 & 1979 & \\
\hline \multirow[t]{8}{*}{ Stapelia gigantea N.E. Br. } & Nancy & 1964 & 1968 & $\begin{array}{l}\text { arrived as S. nobilis; one of } \\
5 \text { synonyms }\end{array}$ \\
\hline & Budapest & 1969 & 1983 & \\
\hline & Goettingen & 1970 & 1971 & \\
\hline & Muenster & 1992 & 2000 & \\
\hline & Liege & 1961 & 1968 & arrived as S. gigantea \\
\hline & Heidelberg & 1962 & 1968 & \\
\hline & Besancon & 1963 & 1966 & \\
\hline & Bucharest & 1964 & 1966 & \\
\hline \multirow[t]{7}{*}{ Stapelia grandiflora Masson } & Bochum & 1995 & 2000 & $\begin{array}{l}\text { arrived as S. ambigua; one } \\
\text { of } 7 \text { synonyms }\end{array}$ \\
\hline & Leipzig & 1955 & no data & arrived as S. grandiflora \\
\hline & nn & $\begin{array}{l}\text { in collection } \\
\text { in } 1961\end{array}$ & 1980 & \\
\hline & Besancon & 1965 & 1968 & \\
\hline & Goettingen & 1965 & 2003 & \\
\hline & Krakow & 1970 & 1978 & \\
\hline & Gent & 1979 & 2000 & \\
\hline Stapelia hanburyana A.Berger \& Ruest & Goettingen & 1965 & 1971 & \\
\hline \multirow[t]{3}{*}{ Stapelia hirsuta L. } & Bucharest & 1964 & 1966 & $10+$ synonyms \\
\hline & Duisburg & $1989 \& 1990$ & $1993 \& 1995$ & \\
\hline & Basel & 1988 & 1993 & \\
\hline Stapelia leendertziae N.E. Br. & Goeteborg & 1978 & 1980 & arrived as S. wilmaniae \\
\hline Stapelia lucida DC. & Duisburg & 1995 & 1997 & 2 synonyms \\
\hline Stapelia $\times$ luxurians Dammann ex Rust & Debrecen & 1956 & 1979 & arrived as S. luxuriana \\
\hline Stapelia mutabilis Jacq. & Hamburg & 1961 & 1962 & 2 synonyms \\
\hline Stapelia mutabilis Jacq. (var. bicolor) & Krakow & $1969 \& 1970$ & $1979 \& 1979$ & $\begin{array}{l}\text { arrived as S. bicolor; } 1 \\
\text { synonym }\end{array}$ \\
\hline Stapelia 'Penzigii' & Uppsala & 1953 & no data & unknown cultivar \\
\hline Stapelia schinzii A. Berger \& Schltr. & Dresden & $1995 \& 1998$ & $2000 \& 1998$ & 4 synonyms \\
\hline Stapelia trifida Tod. & Antwerpen & $\begin{array}{l}1967 \& \\
\text { unknown }\end{array}$ & $1971 \& 1978$ & from cuttings \\
\hline Stapelianthus decaryi Choux & Goettingen & 1970 & 1970 & \\
\hline Tridentea gemmiflora (Masson) Haw. & Besancon & 1968 & 1971 & $\begin{array}{l}\text { arrived as Stapelia } \\
\text { gemmiflora; one of } 3 \\
\text { synonyms; from cuttings }\end{array}$ \\
\hline Tromotriche herrei (Nel) Bruyns & Duisburg & 1994 & 2000 & $\begin{array}{l}\text { arrived as Stapelia herrei; } \\
\text { one of } 4 \text { synonyms }\end{array}$ \\
\hline \multicolumn{5}{|l|}{ Asteraceae } \\
\hline Argyranthemum gracile Sch. Bip. & Oslo & 2006 & 2006 & syn. Chrysanthemum gracile \\
\hline Delairea odorata Lem. & Napoli & 1958 & 1961 & $\begin{array}{l}\text { arrived as Senecio scandens; } \\
\text { one of } 3 \text { synonyms }\end{array}$ \\
\hline Kleinia anteuphorbium (L.) Haw. & nn & unknown & 1963 & 4 synonyms \\
\hline Kleinia longiflora DC. & nn & $\begin{array}{l}\text { in collection } \\
\text { in } 1968\end{array}$ & 1969 & \begin{tabular}{|l|} 
arrived as Senecio \\
longiflorus var.violaceus
\end{tabular} \\
\hline \multirow[t]{4}{*}{ Kleinia neriifolia Haw. } & Basel & 1967 & 1971 & $\begin{array}{l}\text { arrived as Senecio kleinia; } \\
\text { one of } 4 \text { synonyms }\end{array}$ \\
\hline & Braunschweig & 1980 & 1983 & \\
\hline & nn & unknown & 1980 & \\
\hline & Duisburg & 1993 & 2003 & from cuttings \\
\hline Kleinia petraea (R. E. Fr.) C. Jeffrey & Wageningen & $1962 \& 1963$ & 1966 \& 1971 & $\begin{array}{l}\text { arrived as Senecio } \\
\text { jacobsenii; one of } 3 \\
\text { synonyms }\end{array}$ \\
\hline \multirow[t]{2}{*}{ Kleinia stapeliiformis (E. Phillips) Stapf } & Gent & 1961 & 1961 & syn. Senecio stapeliiformis \\
\hline & Besancon & 1971 & 1963 & \\
\hline
\end{tabular}




\begin{tabular}{|c|c|c|c|c|}
\hline Scientific name & $\begin{array}{l}\text { City of plant } \\
\text { material origin }\end{array}$ & $\begin{array}{l}\text { Year of } \\
\text { ordering/ } \\
\text { purchasing }\end{array}$ & $\begin{array}{l}\text { Year when plant } \\
\text { was last recorded } \\
\text { in collection }\end{array}$ & Notes and remarks \\
\hline & Besancon & 1971 & 1971 & $\begin{array}{l}\text { arrived as syn. Senecio } \\
\text { stapeliiformis }\end{array}$ \\
\hline & Lublin & 1996 & 2000 & \\
\hline & Salaspils & 1997 & 2000 & from cuttings \\
\hline \multirow[t]{2}{*}{ Kleinia stapeliiformis (E. Phillips) Stapf (var.minor) } & Rome & 1970 & 1989 & $\begin{array}{l}\text { arrived as syn. Senecio } \\
\text { stapeliiformis (var.minor) }\end{array}$ \\
\hline & Antwerpen & $1970 \& 1971$ & $1971 \& 1971$ & \\
\hline \multirow[t]{5}{*}{ Othonna capensis L.H. Bailey } & Lisbon & 1954 & no data & syn. O. crassifolia \\
\hline & Strassburg & 1962 & 1968 & \\
\hline & Cluj-Napoca & 1974 & 1980 & \\
\hline & Berlin & 2001 & 2002 & \\
\hline & Caen & 2002 & 2003 & \\
\hline Pentacalia caracasana (Klatt) Cuatrec. & Antwerpen & 1967 & 1978 & $\begin{array}{l}\text { arrived as Senecio } \\
\text { cucullatus; from cuttings }\end{array}$ \\
\hline Senecio acaulis (L. f.) Sch. Bip. & Wageningen & 1966 & 1966 & 3 synonyms \\
\hline Senecio angulatus L. f. & Strasbourg & 1966 & 1993 & syn. S. macropodus \\
\hline \multirow{4}{*}{ Senecio articulatus (L. f.) Sch. Bip. } & nn & 1949 & 1971 & 2 synonyms \\
\hline & nn & 1970 & 1980 & \\
\hline & $\mathrm{nn}$ & 1969 & 1971 & \\
\hline & Wageningen & 1972 & 1980 & \\
\hline \multirow[t]{2}{*}{ Senecio citriformis G.D. Rowley } & Wageningen & $\begin{array}{l}1963 \& 1964 \\
\& 1970 \& \\
1980\end{array}$ & $\begin{array}{l}1963 \& 1966 \& \\
1971 \& 1982\end{array}$ & \\
\hline & Gent & 1963 & 1966 & \\
\hline Senecio citrinus (DC.) Voss & Besancon & 1967 & 1968 & from cuttings \\
\hline \multirow[t]{2}{*}{ Senecio crassissimus Humbert } & Genova & $1963 \& 1970$ & $1963 \& 1971$ & \\
\hline & Wageningen & $1963 \& 1964$ & $1963 \& 1966$ & \\
\hline Senecio cuneatus Hook. f. & Goeteborg & 1970 & 1971 & 2 synonyms \\
\hline \multirow[t]{2}{*}{ Senecio cylindricus (A.Berger) Jacobsen } & Gent & 1960 & 2000 & \\
\hline & Dresden & 2000 & 2003 & \\
\hline \multirow[t]{3}{*}{ Senecio kleiniiformis Suess. } & Wageningen & $\begin{array}{l}1963 \& 1964 \\
\& 1970\end{array}$ & $\begin{array}{l}1966 \& 1966 \& \& \\
1979\end{array}$ & \\
\hline & Gent & $1960 \& 1961$ & $1966 \& 1966$ & \\
\hline & nn & unknown & 1968 & \\
\hline Senecio macroglossus DC. & Pisa & $1966 \& 1973$ & $1979 \& 1993$ & \\
\hline \multirow[t]{2}{*}{ Senecio mandraliscae (Tineo) H.Jacobsen } & Wageningen & $\begin{array}{l}1960 \& 1963 \\
\& 1964\end{array}$ & $\begin{array}{l}1966 \& 1966 \& \text { \& } \\
1966\end{array}$ & \\
\hline & Genova & 1970 & 1971 & \\
\hline Senecio medley-woodii Hutch. & Antwerpen & $\begin{array}{l}1966 \& 1967 \\
\& 1968 \& \\
1970\end{array}$ & $\begin{array}{l}1968 \& 2010 \& \\
1971 \& 1970\end{array}$ & from cuttings (1967) \\
\hline Senecio oxyriifolius DC. & Antwerpen & $1969 \& 1970$ & $2010 \& 1989$ & 5 synonyms \\
\hline \multirow[t]{2}{*}{ Senecio radicans (L. f.) Sch. Bip. } & Besancon & 1962 & 1963 & 3 synonyms \\
\hline & Wageningen & 1963 & 1963 & \\
\hline \multirow[t]{2}{*}{ Senecio repens (L.) H.Jacobsen } & Lisbon & 1954 & no data & arrived as Kleinia repens \\
\hline & Gent & 1960 & 1966 & \\
\hline \multirow[t]{2}{*}{ Senecio rowleyanus H.Jacobsen } & Goeteborg & $\begin{array}{l}1970 \& 1971 \\
\& 1978\end{array}$ & $\begin{array}{l}1982 \& 1979 \& \text { \& } \\
1989\end{array}$ & \\
\hline & Dresden & 1998 & 2000 & from cuttings \\
\hline Senecio scaposus DC. & Wageningen & 1962 & 1966 & 2 synonyms \\
\hline Senecio serpens G.D. Rowley & Rome & 1970 & 1970 & 2 synonyms \\
\hline Senecio tropaeolifolius MacOwan ex F.Muell. & Antwerpen & 1970 & 1989 & \\
\hline \multicolumn{5}{|l|}{ Cactaceae } \\
\hline \multirow[t]{6}{*}{ Acanthocalycium spiniflorum (K. Schum.) Backeb. } & Linz & $1986 \& 2008$ & $2000 \& 2009$ & $\begin{array}{l}\text { arrived as Echinopsis } \\
\text { spiniflora; one of } 2 \\
\text { synonyms }\end{array}$ \\
\hline & Debrecen & 1989 & 2000 & \\
\hline & Bratislava & 2000 & 2003 & \\
\hline & Palermo & 1962 & 1972 & $\begin{array}{l}\text { arrived as Echinocactus } \\
\text { spiniflorus }\end{array}$ \\
\hline & Halle & 1967 & 1977 & arrived as $A$. violaceum \\
\hline & Linz & $1967 \& 1986$ & $1977 \& 2000$ & \\
\hline
\end{tabular}


S16 Sandev, D. et al.: Plethora of plants - collections of the Botanical Garden, Faculty of Science, University of Zagreb (2)

\begin{tabular}{|c|c|c|c|c|}
\hline Scientific name & $\begin{array}{l}\text { City of plant } \\
\text { material origin }\end{array}$ & $\begin{array}{l}\text { Year of } \\
\text { ordering/ } \\
\text { purchasing }\end{array}$ & $\begin{array}{l}\text { Year when plant } \\
\text { was last recorded } \\
\text { in collection }\end{array}$ & Notes and remarks \\
\hline \multirow[t]{2}{*}{ Acanthocalycium thionanthum (Speg.) Backeb. } & Linz & 1976 & 1983 & $\begin{array}{l}\text { arrived as Echinopsis } \\
\text { thionantha; one of } 7+\text { syno- } \\
\text { nyms }\end{array}$ \\
\hline & Debrecen & 1989 & 2000 & \\
\hline \multirow[t]{4}{*}{ Acanthocereus tetragonus (L.) Hummelinck } & nn & 1955 & no data & $\begin{array}{l}\text { arrived as Cereus pitahaya; } \\
\text { from cuttings; one of } 20+ \\
\text { synonyms }\end{array}$ \\
\hline & nn & $\begin{array}{l}\text { in collection } \\
\text { in } 1961 \text { \& in } \\
\text { collection in } \\
1980\end{array}$ & $1977 \& 1990$ & from cuttings (1961) \\
\hline & Barcelona & 1968 & 1973 & \\
\hline & Porto & 1971 & 1990 & \\
\hline $\begin{array}{l}\text { Acantholobivia incuiensis (Rauh \& Backeb.) Rauh } \\
\text { \& Backeb. }\end{array}$ & $\begin{array}{l}\text { Vienna-Belve- } \\
\text { dere }\end{array}$ & 1971 & 1978 & $\begin{array}{l}2 \text { synonyms; unresolved } \\
\text { name (the Plant List) }\end{array}$ \\
\hline \multirow[t]{2}{*}{ Acantholobivia tegeleriana (Backeb.) Backeb. } & Linz & 1972 & no data & $\begin{array}{l}2 \text { synonyms; unresolved } \\
\text { name (the Plant List) }\end{array}$ \\
\hline & Berlin & 1968 & 1975 & \\
\hline Armatocereus rauhii Backeb. & Liege & 1973 & 1998 & 2 synonyms \\
\hline \multirow[t]{5}{*}{ Astrophytum asterias (Zucc.) Lem. } & Tuebingen & 1963 & 1963-1970 & $10+$ synonyms \\
\hline & Debrecen & $1980 \& 1992$ & $1983 \& 1993$ & \\
\hline & Linz & 1991 & no data & \\
\hline & Chemnitz & $1992 \& 1993$ & $1993 \& 2000$ & \\
\hline & Frankfurt & 1992 & 1993 & \\
\hline \multirow[t]{8}{*}{ Astrophytum capricorne (A.Dietr.) Britton \& Rose } & Tuebingen & 1963 & 1979 & $20+$ synonyms \\
\hline & Dresden & 1970 & 1980 & \\
\hline & Linz & $1987 \& 2003$ & 1990 \& 2006 & \\
\hline & Frankfurt & 1992 & 1993 & \\
\hline & Chemnitz & 1992 & 2010 & \\
\hline & Debrecen & 1992 & 2010 & \\
\hline & Debrecen & 1992 & 2000 & $\begin{array}{l}\text { arrived as } A . \text { senile; one of } \\
20+\text { synonyms }\end{array}$ \\
\hline & Frankfurt & 1992 & 2000 & \\
\hline \multirow[t]{23}{*}{ Astrophytum myriostigma Lem. } & Berlin & 1965 & 1983 & $\begin{array}{l}\text { arrived as A. coahuilense; } \\
\text { one of } 20+\text { synonyms }\end{array}$ \\
\hline & Vienna & 1966 & 1968 & \\
\hline & Linz & $1963 \& 1966$ & $1966 \& 1968$ & $\begin{array}{l}\text { Astrophytum myriostigma } \\
\text { var. glabrum }\end{array}$ \\
\hline & Dresden & 1971 & 1982 & \\
\hline & Barcelona & 1961 & 1968 & \\
\hline & Halle & 1957 & 1968 & \\
\hline & Berlin & $1958 \& 1966$ & $1968 \& 1968$ & \\
\hline & Vienna & 1963 & 1968 & \\
\hline & Basel & 1963 & 1968 & \\
\hline & Karlsruhe & 1978 & 1990 & \\
\hline & Lokrum & 1968 & 1983 & $\begin{array}{l}\text { arrived as A. myriostigma } \\
\text { var. columnare }\end{array}$ \\
\hline & Sarvar & 1973 & 1983 & $\begin{array}{l}\text { arrived as A. myriostigma } \\
\text { var. potosinum }\end{array}$ \\
\hline & Basel & 1973 & 1989 & \\
\hline & Linz & 1976 & 1980 & \\
\hline & Berlin & 1974 & 1980 & $\begin{array}{l}\text { arrived as A. myriostigma } \\
\text { var. quadricostatum }\end{array}$ \\
\hline & Lokrum & 1970 & 1982 & $\begin{array}{l}\text { arrived as A. myriostigma } \\
\text { var. strongylogonum }\end{array}$ \\
\hline & Linz & $1963 \& 1966$ & $1966 \& 1968$ & $\begin{array}{l}\text { arrived as A. myriostigma } \\
\text { var. strongylogonum subv. } \\
\text { nudum }\end{array}$ \\
\hline & Dresden & 1971 & 1982 & \\
\hline & Barcelona & 1961 & 1968 & \\
\hline & Halle & 1957 & 1968 & \\
\hline & Berlin & $1958 \& 1966$ & $1968 \& 1968$ & \\
\hline & Vienna & 1963 & 1968 & \\
\hline & Basel & 1963 & 1968 & \\
\hline
\end{tabular}




\begin{tabular}{|c|c|c|c|c|}
\hline Scientific name & $\begin{array}{l}\text { City of plant } \\
\text { material origin }\end{array}$ & $\begin{array}{l}\text { Year of } \\
\text { ordering/ } \\
\text { purchasing }\end{array}$ & $\begin{array}{l}\text { Year when plant } \\
\text { was last recorded } \\
\text { in collection }\end{array}$ & Notes and remarks \\
\hline & Karlsruhe & 1978 & 1990 & \\
\hline & Halle & $1957 \& 1967$ & $1972 \& 1977$ & arrived as A. myriostigma \\
\hline & Barcelona & $1961 \& 1984$ & $1983 \& 1990$ & \\
\hline & Basel & 1963 & 1979 & \\
\hline & Linz & $\begin{array}{l}1963 \& 1966 \\
\& 1972 \& \\
1976\end{array}$ & $\begin{array}{l}1972 \& 1990 \& \\
1990 \& 1983\end{array}$ & \\
\hline & Vienna & $1966 \& 1970$ & $1972 \& 1993$ & \\
\hline & Lokrum & 1970 & 1990 & \\
\hline & Gent & 1968 & 1975 & \\
\hline & nn & 1972 & 1990 & \\
\hline & Dresden & 1971 & 1975 & \\
\hline & Muenchen & 1972 & 1975 & \\
\hline & Kamani & 1975 & 1983 & \\
\hline & Karlsruhe & 1978 & 1982 & \\
\hline \multirow[t]{6}{*}{ Astrophytum ornatum (DC.) Britton \& Rose } & Barcelona & $\begin{array}{l}1959 \& 1961 \\
\& 1967 \& \\
1971 \& 1970 \\
\& 1972 \& \\
1994\end{array}$ & $\begin{array}{l}2016 \& 1983 \& \\
2016 \& 1983 \& \\
1983 \& 1978 \& \\
1996\end{array}$ & $15+$ synonyms \\
\hline & Palermo & 1961 & 1962 & \\
\hline & Gotingen & $1965 \& 1971$ & $1972 \& 1978$ & \\
\hline & Tubingen & 1970 & 1975 & \\
\hline & Karlsruhe & 1978 & 1993 & \\
\hline & Kiel & 1991 & 1993 & \\
\hline \multirow[t]{5}{*}{ Austrocylindropuntia cylindrica (Lam.) Backeb. } & Barcelona & 1963 & 1982 & 3 synonyms \\
\hline & nn & $\begin{array}{l}\text { in collection } \\
\text { in } 1972\end{array}$ & 1987 & \\
\hline & nn & 1952 & no data & $\begin{array}{l}\text { arrived as Opuntia } \\
\text { cylindrica; from cuttings }\end{array}$ \\
\hline & Lisbon & $1954 \& 1955$ & no data \& 1975 & from cuttings (1954) \\
\hline & Naples & 1962 & 1968 & \\
\hline $\begin{array}{l}\text { Austrocylindropuntia floccosa (Salm-Dyck ex } \\
\text { Winterfeld) F.Ritter }\end{array}$ & Halle & 1966 & $1966-1974$ & $\begin{array}{l}\text { 10+ synonyms; from } \\
\text { cuttings }\end{array}$ \\
\hline \multirow[t]{3}{*}{$\begin{array}{l}\text { Austrocylindropuntia salmiana (Parm. ex Pfeiff.) } \\
\text { Backeb. }\end{array}$} & Uppsala & 1958 & 1975 & $\begin{array}{l}\text { arrived as A. salmiana var. } \\
\text { spegazzinii; one of } 5 \\
\text { synonyms }\end{array}$ \\
\hline & Goeteborg & 1986 & no data & \\
\hline & Liege & 1986 & 1987 & arrived as A. salmiana; \\
\hline \multirow[t]{4}{*}{$\begin{array}{l}\text { Austrocylindropuntia subulata (Muehlenpf.) } \\
\text { Backeb. }\end{array}$} & nn & unknown & 1961 & 7 synonyms \\
\hline & Vienna & 1964 & 1964 & from cuttings \\
\hline & Barcelona & 1964 & 1964 & \\
\hline & Liege & 1986 & 1989 & \\
\hline $\begin{array}{l}\text { Austrocylindropuntia verschaffeltii (Cels ex } \\
\text { F.A.C.Weber) Backeb. }\end{array}$ & Barcelona & $1961 \& 1963$ & $1982 \& 1979$ & $\begin{array}{l}\text { arrived as Opuntia } \\
\text { verschaffeltii; one of } 10+ \\
\text { synonyms }\end{array}$ \\
\hline \multirow[t]{2}{*}{ Austrocylindropuntia vestita (Salm-Dyck) Backeb. } & Beograd & 1965 & 1968 & $5+$ synonyms \\
\hline & Barcelona & 1971 & 1972 & \\
\hline \multirow[t]{2}{*}{ Aylostera deminuta (F.A.C.Weber) Backeb. } & Jibou & 1998 & 2000 & $\begin{array}{l}\text { unresolved name (The } \\
\text { Plant List); } 2 \text { synonyms }\end{array}$ \\
\hline & Cluj & 2000 & 2003 & \\
\hline \multirow[t]{4}{*}{ Aylostera fiebrigii (Gürke) Backeb. } & Sankt Peterburg & 1959 & 1961 & $\begin{array}{l}\text { unresolved name (The } \\
\text { Plant List); } 2 \text { synonyms }\end{array}$ \\
\hline & Gent & 1966 & 1978 & \\
\hline & Lokrum & 1970 & 1975 & \\
\hline & Goettingen & 1989 & 1990 & \\
\hline \multirow[t]{6}{*}{ Aylostera kupperiana (Boed.) Backeb. } & Berlin & 1955 & 1966 & $\begin{array}{l}\text { unresolved name (The } \\
\text { Plant List); } 2 \text { synonyms }\end{array}$ \\
\hline & Gent & 1963 & 1966 & \\
\hline & Karlsruhe & 1964 & 1978 & \\
\hline & Sarvar & 1973 & 1983 & \\
\hline & Vienna & 1971 & 1983 & \\
\hline & Dresden & 1986 & 1990 & \\
\hline
\end{tabular}


S18 Sandev, D. et al.: Plethora of plants - collections of the Botanical Garden, Faculty of Science, University of Zagreb (2)

\begin{tabular}{|c|c|c|c|c|}
\hline Scientific name & $\begin{array}{l}\text { City of plant } \\
\text { material origin }\end{array}$ & $\begin{array}{l}\text { Year of } \\
\text { ordering/ } \\
\text { purchasing }\end{array}$ & $\begin{array}{l}\text { Year when plant } \\
\text { was last recorded } \\
\text { in collection }\end{array}$ & Notes and remarks \\
\hline \multirow[t]{8}{*}{ Aylostera pseudodeminuta (Backeb) Backeb. } & Halle & 1961 & 1975 & $\begin{array}{l}\text { unresolved name (The } \\
\text { Plant List); } 3 \text { synonyms }\end{array}$ \\
\hline & nn & $\begin{array}{l}\text { in collection } \\
\text { in } 1972\end{array}$ & 1978 & \\
\hline & Gent & 1968 & 1979 & \\
\hline & Linz & 1972 & 1983 & \\
\hline & Debrecen & 1980 & 1989 & \\
\hline & Gent & 1965 & 1972 & $\begin{array}{l}\text { arrived as A.pseudodem- } \\
\text { inuta var. grandiflora }\end{array}$ \\
\hline & nn & unknown & 1978 & \\
\hline & Debrecen & 1980 & 1983 & \\
\hline $\begin{array}{l}\text { Aylostera pseudodeminuta var. schumanniana } \\
\text { Backeb. }\end{array}$ & Linz & 1965 & 1978 & syn. Rebutia deminuta \\
\hline \multirow[t]{2}{*}{ Aylostera spegazziniana (Backeb.) Backeb. } & Halle & 1964 & 1975 & $\begin{array}{l}\text { syn. Rebutia spegazziniana; } \\
\text { unresolved name (The } \\
\text { Plant List) }\end{array}$ \\
\hline & Turku & 1967 & 1979 & \\
\hline Aylostera spinosissima (Backeb.) Backeb. & Vienna & 1963 & 1968 & $\begin{array}{l}\text { syn. Rebutia spinosissima; } \\
\text { unresolved name (The } \\
\text { Plant List) }\end{array}$ \\
\hline Bergerocactus emoryi (Engelm.) Britton \& Rose & $\begin{array}{l}\text { San Marino } \\
\text { (California) }\end{array}$ & 1984 & 1987 & 2 synonyms \\
\hline $\begin{array}{l}\text { Borzicactus aurantiacus (Vaupel) Kimnach \& } \\
\text { Hutchison }\end{array}$ & Monaco & 1997 & 2003 & $\begin{array}{l}\text { arrived as Matucana } \\
\text { weberbaueri; one of } 20+ \\
\text { synonyms }\end{array}$ \\
\hline \multirow[t]{5}{*}{$\begin{array}{l}\text { Borzicactus celsianus (Lem. ex Salm-Dyck) } \\
\text { Kimnach }\end{array}$} & nn & $\begin{array}{l}\text { in collection } \\
\text { in } 1968\end{array}$ & 1980 & $\begin{array}{l}\text { arrived as Oreocereus } \\
\text { neocelsianus; } 20+ \\
\text { synonyms }\end{array}$ \\
\hline & Lokrum & 1968 & 2010 & \\
\hline & Barcelona & 1971 & 1980 & \\
\hline & Monaco & 1996 & 2010 & \\
\hline & Berlin & 1973 & 1989 & $\begin{array}{l}\text { arrived as Cleistocactus } \\
\text { tupizensis }\end{array}$ \\
\hline \multirow[t]{2}{*}{ Borzicactus haynei (Otto ex Salm-Dyck) Kimnach } & Halle & 1973 & 1983 & $\begin{array}{l}\text { arrived as Matucana } \\
\text { haynei; one of } 3 \text { synonyms }\end{array}$ \\
\hline & Linz & 1975 & 1983 & $\begin{array}{l}\text { arrived as Matucana } \\
\text { yunganucensis }\end{array}$ \\
\hline \multirow[t]{2}{*}{ Brasilicactus graessneri (K.Schum.) Backeb. } & Berlin & 1955 & 1980 & $\begin{array}{l}\text { unresolved name (The } \\
\text { Plant List); syn. Parodia } \\
\text { haselbergii subsp. graessneri }\end{array}$ \\
\hline & Kiev & 1986 & 1990 & \\
\hline \multirow[t]{4}{*}{$\begin{array}{l}\text { Brasilicactus haselbergii (Haage ex Rumpl.) Backeb. } \\
\text { ex Jul.Schäff. }\end{array}$} & Berlin & 1955 & 1966 & $\begin{array}{l}\text { unresolved name (The } \\
\text { Plant List); syn. Parodia } \\
\text { haselbergii }\end{array}$ \\
\hline & Beograd & 1965 & 1966 & \\
\hline & Barcelona & 1971 & 1977 & \\
\hline & Kiev & 1986 & 1990 & \\
\hline \multirow[t]{3}{*}{ Brasiliopuntia brasiliensis (Willd.) A.Berger } & Szeged & 1960 & 1980 & 10+ synonyms \\
\hline & Monaco & 1989 & 1993 & \\
\hline & Barcelona & $\begin{array}{l}1962 \& 1963 \\
\& 1964\end{array}$ & $\begin{array}{l}1963 \& 1972 \& \& \\
1983\end{array}$ & arrived as B. bahiensis \\
\hline Browningia candelaris (Meyen) Britton \& Rose & Liege & 1973 & 1983 & 2 synonyms \\
\hline Browningia hertlingiana (Backeb.) Buxb. & Berkeley & 1959 & 1978 & $\begin{array}{l}\text { arrived as Azureocereus } \\
\text { hertlingianus; one of } 3 \\
\text { synonyms }\end{array}$ \\
\hline Cactus glaucus Kuntze & Palermo & $\begin{array}{l}1957 \& 1959 \\
\& 1962\end{array}$ & $\begin{array}{l}1983 \& 1977 \& \\
2000\end{array}$ & $\begin{array}{l}\text { arrived as Mammillaria } \\
\text { glauca }\end{array}$ \\
\hline Cactus krameri Kuntze & Palermo & 1962 & 1993 & $\begin{array}{l}\text { arrived as Mammillaria } \\
\text { krameri }\end{array}$ \\
\hline \multirow{4}{*}{ Carnegiea gigantea (Engelm.) Britton \& Rose } & Bucharest & 1964 & 1993 & 3 synonyms \\
\hline & Bochum & 1995 & 2000 & \\
\hline & Bucharest & $1957 \& 1964$ & $1972 \& 1990$ & arrived as Cereus giganteus \\
\hline & $\begin{array}{l}\text { Davis } \\
\text { (California) }\end{array}$ & 1980 & 1990 & \\
\hline
\end{tabular}




\begin{tabular}{|c|c|c|c|c|}
\hline Scientific name & $\begin{array}{l}\text { City of plant } \\
\text { material origin }\end{array}$ & $\begin{array}{l}\text { Year of } \\
\text { ordering/ } \\
\text { purchasing }\end{array}$ & $\begin{array}{l}\text { Year when plant } \\
\text { was last recorded } \\
\text { in collection }\end{array}$ & Notes and remarks \\
\hline \multirow[t]{2}{*}{ Cephalocereus senilis (Haw.) Pfeiff. } & Lokrum & $\begin{array}{l}1967 \& 1968 \\
\& 1970\end{array}$ & $\begin{array}{l}1970 \& 1970 \& \\
1993\end{array}$ & $\begin{array}{l}\text { 10+ synonyms; from } \\
\text { cuttings (1967) }\end{array}$ \\
\hline & nn & 1947 & 1963 & $\begin{array}{l}\text { arrived as Cereus senilis; } \\
\text { arrived as planta viva }\end{array}$ \\
\hline Cereus acutangulus Pfeiff. & Gent & 1968 & 1988 & $\begin{array}{l}\text { arrived as Acanthocereus } \\
\text { acutangulus; unresolved } \\
\text { name (the Plant List) }\end{array}$ \\
\hline Cereus alacriportanus Pfeiff. & Vienna & $1955 \& 1956$ & 1966 \& 1990 & $\begin{array}{l}\text { unresolved name (The } \\
\text { Plant List) }\end{array}$ \\
\hline \multirow{2}{*}{ Cereus azureus J. Parm. ex Pfeiff. } & Erlangen & 1961 & 1990 & syn. C. aethiops \\
\hline & Barcelona & 1967 & 1989 & \\
\hline Cereus braunii Cárdenas & Barcelona & 1964 & 1989 & \\
\hline Cereus chalybaeus Otto ex Walp. & Karlsruhe & 1965 & 1990 & \\
\hline Cereus comarapanus Cárdenas & $\begin{array}{l}\text { Vienna-Belve- } \\
\text { dere }\end{array}$ & 1971 & 1993 & $\begin{array}{l}\text { syn. Piptanthocereus } \\
\text { comarapanus }\end{array}$ \\
\hline \multirow[t]{2}{*}{ Cereus hildmannianus K. Schum. } & Barcelona & 1980 & 1993 & $\begin{array}{l}\text { arrived as C. xanthocarpus; } \\
\text { one of } 40+\text { synonyms }\end{array}$ \\
\hline & nn & $\begin{array}{l}\text { in collection } \\
\text { in } 1961\end{array}$ & 1972 & arrived as C. monstrosus \\
\hline \multirow[t]{5}{*}{ Cereus jamacaru DC. } & Vacratot & 1961 & 1961 & 8 synonyms \\
\hline & Barcelona & 1967 & 1993 & \\
\hline & Muenchen & 1977 & 1990 & \\
\hline & Wuppertal & 1984 & 1989 & \\
\hline & Szeged & 1985 & 2016 & \\
\hline $\begin{array}{l}\text { Cereus paraguayensis K.Schum. ex Chodat \& } \\
\text { Hassl. }\end{array}$ & Muenchen & 1959 & 1970 & \\
\hline Cereus pitajaya DC. "var. monstrosus hort." & nn & $\begin{array}{l}\text { in collection } \\
\text { in } 1967\end{array}$ & 1993 & \begin{tabular}{|l|} 
syn. Acanthocereus \\
tetragonus
\end{tabular} \\
\hline \multirow[t]{2}{*}{ Cereus repandus (L.) Mill. } & nn & unknown & 1961 & $\begin{array}{l}\text { arrived as C. peruvianus; } \\
\text { one of } 20+\text { synonyms }\end{array}$ \\
\hline & Palermo & 2001 & 2003 & \\
\hline \multirow[t]{2}{*}{ Cereus repandus (L.) Mill. f.monstrosa DC. } & nn & $\begin{array}{l}\text { in collection } \\
\text { in } 1961\end{array}$ & 2016 & $\begin{array}{l}\text { arrived as C. peruvianus } \mathrm{f} \text {. } \\
\text { monstrosa }\end{array}$ \\
\hline & Blanes & 1994 & 2010 & \\
\hline Cereus validus Haw. & nn & 1951 & 1989 & syn. C. forbesii hort. \\
\hline Cereus vargasianus Cárdenas & $\begin{array}{l}\text { Vienna-Belve- } \\
\text { dere }\end{array}$ & 1971 & 1983 & \\
\hline Cleistocactus acanthurus (Vaupel) D.R.Hunt & Liege & 1973 & 1982 & 3 synonyms \\
\hline \multirow{3}{*}{ Cleistocactus baumannii (Lem.) Lem. } & Palermo & 1964 & 1970 & 2 synonyms \\
\hline & Halle & $1965 \& 1966$ & $1978 \& 1972$ & \\
\hline & Berlin & 1987 & 1990 & \\
\hline $\begin{array}{l}\text { Cleistocactus baumannii subsp. chacoanus (F.Ritter) } \\
\text { P.J.Braun \& Esteves }\end{array}$ & Wageningen & 1972 & 1990 & arrived as C. margaritanus \\
\hline \multirow[t]{2}{*}{$\begin{array}{l}\text { Cleistocactus baumannii subsp. santacruzensis } \\
\text { (Backeb.) Mottram }\end{array}$} & Halle & 1967 & 1975 & arrived as C. anguinus \\
\hline & Wageningen & 1972 & 1993 & arrived as C. grossei \\
\hline Cleistocactus candelilla Cárdenas & Liege & 1984 & 2000 & 6 synonyms \\
\hline $\begin{array}{l}\text { Cleistocactus fieldianus (Britton \& Rose) D.R.Hunt } \\
\text { subsp. tessellatus (Akers \& Buining) Ostolaza }\end{array}$ & Barcelona & 1980 & 1983 & $\begin{array}{l}\text { arrived as Clistanthocereus } \\
\text { tesselatus; one of } 4 \\
\text { synonyms }\end{array}$ \\
\hline \multirow[t]{2}{*}{ Cleistocactus hyalacanthus (K.Schum.) Rol.-Goss. } & Lokrum & 1969 & 1977 & arrived as C. jujuyensis \\
\hline & Barcelona & 1971 & 1993 & \\
\hline $\begin{array}{l}\text { Cleistocactus hyalacanthus subsp. tarijensis } \\
\text { (Cárdenas) Mottram }\end{array}$ & Linz & 1965 & 1993 & arrived as $C$. tarijensis \\
\hline Cleistocactus icosagonus (Kunth) F.A.C. Weber & nn & 1951 & 1982 & 4 synonyms \\
\hline Cleistocactus parviflorus (K.Schum.) Rol.-Goss. & $\begin{array}{l}\text { Vienna-Belve- } \\
\text { dere }\end{array}$ & 1971 & 1993 & arrived as C. herzogianus \\
\hline Cleistocactus sepium (Kunth) A.Weber & Barcelona & 1980 & 1990 & $\begin{array}{l}\text { arrived as Borzicactus } \\
\text { morleyanus }\end{array}$ \\
\hline \multirow[t]{2}{*}{$\begin{array}{l}\text { Cleistocactus smaragdiflorus (F.A.C.Weber) Britton } \\
\& \text { Rose. }\end{array}$} & Palermo & 1964 & 1989 & $\begin{array}{l}\text { arrived as C. baumannii } \\
\text { var. smaragdiflora; one of } 2 \\
\text { synonyms }\end{array}$ \\
\hline & Freiburg & 1957 & 1982 & $\begin{array}{l}\text { arrived as Cereus } \\
\text { smaragdiflorus }\end{array}$ \\
\hline
\end{tabular}


S20 Sandev, D. et al.: Plethora of plants - collections of the Botanical Garden, Faculty of Science, University of Zagreb (2)

\begin{tabular}{|c|c|c|c|c|}
\hline Scientific name & $\begin{array}{l}\text { City of plant } \\
\text { material origin }\end{array}$ & $\begin{array}{l}\text { Year of } \\
\text { ordering/ } \\
\text { purchasing }\end{array}$ & $\begin{array}{l}\text { Year when plant } \\
\text { was last recorded } \\
\text { in collection }\end{array}$ & Notes and remarks \\
\hline \multirow[t]{9}{*}{ Cleistocactus straussii (Heese) Backeb. } & Vienna & $1968 \& 1995$ & $1983 \& 2003$ & $\begin{array}{l}\text { arrived as C. straussii var. } \\
\text { fricii; one of } 5 \text { synonyms }\end{array}$ \\
\hline & Halle & $1967 \& 1969$ & 1973 \& no data & arrived as C. straussii \\
\hline & Vienna & 1968 & 2000 & \\
\hline & Goettingen & 1966 & 2000 & \\
\hline & Tuebingen & 1963 & 1972 & \\
\hline & Erlangen & 1965 & 1966 & \\
\hline & Muenchen & 1964 & 1972 & \\
\hline & Linz & 1964 & 1978 & \\
\hline & Berlin & 1973 & 1977 & \\
\hline \multirow[t]{2}{*}{ Cleistocactus tupizensis (Vaupel) Backeb. } & Barcelona & $1955 \& 1965$ & $1968 \& 1968$ & syn. Cereus tupizensis \\
\hline & nn & unknown & 1968 & \\
\hline Cleistocactus winteri D.R.Hunt & Cluj-Napoca & 2005 & 2006 & 4 synonyms \\
\hline Copiapoa humilis (Phil.) Hutchison & Budapest & 1978 & 1980 & 4 synonyms \\
\hline Copiapoa hypogaea F.Ritter & Debrecen & 1980 & 1983 & syn. C. barquitensis \\
\hline Corynopuntia dumetorum (A.Berger) F.M.Knuth & Barcelona & 1980 & 1983 & 2 synonyms \\
\hline Corynopuntia emoryi (Engelm.) M.P.Griff. & Barcelona & $\begin{array}{l}1968 \& 1969 \\
\& 1970\end{array}$ & $\begin{array}{l}1978 \& 1980 \& \\
1980\end{array}$ & $\begin{array}{l}\text { arrived as C. stanlyi; } 3 \\
\text { synonyms }\end{array}$ \\
\hline Corynopuntia vilis (Rose) F.M.Knuth & nn & $\begin{array}{l}\text { in collection } \\
\text { in } 1963\end{array}$ & 1963 & 3 synonyms \\
\hline \multirow[t]{5}{*}{ Coryphantha cornifera (DC.) Lem. } & Berlin & 1958 & 1978 & $\begin{array}{l}\text { syn. Mammillaria cornifera; } \\
\text { one of } 3 \text { synonyms }\end{array}$ \\
\hline & Monaco & 1989 & 1990 & \\
\hline & Berlin & 1968 & 1982 & arrived as C. schwarziana \\
\hline & Palermo & 1957 & 1990 & $\begin{array}{l}\text { arrived as Mammillaria } \\
\text { cornifera }\end{array}$ \\
\hline & nn & 1962 & 1964 & \\
\hline Coryphantha echinus (Engelm.) Orcutt & Gent & 1963 & no data & 2 synonyms \\
\hline \multirow[t]{2}{*}{ Coryphantha elephantidens (Lem.) Lem. } & Berlin & 1961 & 1961 & $\begin{array}{l}\text { syn. Mammillaria } \\
\text { elephantidens }\end{array}$ \\
\hline & Halle & 1973 & 1977 & arrived as C. sulcolanata \\
\hline $\begin{array}{l}\text { Coryphantha elephantidens subsp. bumamma } \\
\text { (Ehrenb.) Dicht \& A.Lüthy }\end{array}$ & Budapest & 1978 & 1979 & arrived as C. bumamma \\
\hline Coryphantha erecta (Lem. ex Pfeiff.) Lem. & Barcelona & 1961 & 1983 & syn. Mammillaria erecta \\
\hline Coryphantha octacantha (DC.) Britton \& Rose & Palermo & 1957 & 1993 & $\begin{array}{l}\text { arrived as Mammillaria } \\
\text { macrothele; one of } 3 \\
\text { synonyms }\end{array}$ \\
\hline Coryphantha odorata Boed. & Debrecen & 1980 & 1983 & 2 synonyms \\
\hline Coryphantha pycnacantha (Mart.) Lem. & Berlin & 1960 & 1961 & arrived as $C$. andreae \\
\hline Coryphantha retusa (Pfeiff) Britton \& Rose & Dresden & 1998 & 2000 & 3 synonyms \\
\hline Coryphantha vivipara (Nutt.) Britton \& Rose & Muenster & 1968 & 1975 & $\begin{array}{l}\text { arrived as C. vivipara var. } \\
\text { arizonica; syn. Escobaria } \\
\text { vivipara }\end{array}$ \\
\hline Cumulopuntia sphaerica (Foerster) E.F. Anderson & nn & 1963 & 1983 & $\begin{array}{l}\text { arrived as Tephrocactus } \\
\text { sphaerica; one of } 10+ \\
\text { synonyms }\end{array}$ \\
\hline $\begin{array}{l}\text { Cylindropuntia californica (Torr. \& A.Gray) } \\
\text { F.M.Knuth }\end{array}$ & Barcelona & 1964 & 1983 & 2 synonyms \\
\hline $\begin{array}{l}\text { Cylindropuntia californica subsp. parkeri } \\
\text { (J.M.Coult.) U.Guzmán }\end{array}$ & Antwerpen & 1967 & 1968 & $\begin{array}{l}\text { arrived as Opuntia } \\
\text { bernardina fa.cristata; one } \\
\text { of } 4 \text { synonyms; from } \\
\text { cuttings }\end{array}$ \\
\hline $\begin{array}{l}\text { Cylindropuntia californica subsp. rosarica (G.E. } \\
\text { Linds.) U. Guzmán }\end{array}$ & Barcelona & 1960 & 1968 & 3 synonyms \\
\hline \multirow[t]{2}{*}{$\begin{array}{l}\text { Cylindropuntia echinocarpa (Engelm. \& J.M. } \\
\text { Bigelow) F.M. Knuth }\end{array}$} & Berkeley & 1994 & 2003 & $\begin{array}{l}2 \text { synonyms; arrived as } \\
\text { Opuntia echinocarpa }\end{array}$ \\
\hline & Barcelona & 1964 & 1968 & \\
\hline \multirow[t]{3}{*}{ Cylindropuntia imbricata (Haw.) F.M.Knuth } & nn & unknown & 1968 & 8 synonyms \\
\hline & Bucharest & 1963 & 1963 & $\begin{array}{l}\text { arrived as Opuntia } \\
\text { arborescens }\end{array}$ \\
\hline & Lisle (USA) & $1987 \& 1989$ & $1993 \& 2003$ & \\
\hline \multirow[t]{3}{*}{ Cylindropuntia kleiniae (DC.) F.M.Knuth } & Barcelona & 1963 & 1975 & syn. Opuntia kleiniae \\
\hline & Esperanca & 1970 & 1980 & \\
\hline & Liege & 1984 & 1970 & \\
\hline
\end{tabular}




\begin{tabular}{|c|c|c|c|c|}
\hline Scientific name & $\begin{array}{l}\text { City of plant } \\
\text { material origin }\end{array}$ & $\begin{array}{l}\text { Year of } \\
\text { ordering/ } \\
\text { purchasing }\end{array}$ & $\begin{array}{l}\text { Year when plant } \\
\text { was last recorded } \\
\text { in collection }\end{array}$ & Notes and remarks \\
\hline \multirow[t]{6}{*}{ Cylindropuntia leptocaulis (DC.) F.M. Knuth } & Rome & 1970 & 1982 & $\begin{array}{l}\text { arrived as } C \text {. leptocaulis } \\
\text { var. vaginata; } 2 \text { synonyms }\end{array}$ \\
\hline & Barcelona & $1961 \& 1962$ & $1979 \& 1982$ & \\
\hline & nn & $\begin{array}{l}\text { in collection } \\
\text { in } 1968\end{array}$ & 1972 & \\
\hline & Rotterdam & 1969 & 1986 & \\
\hline & Prague & 1989 & 1990 & \\
\hline & Barcelona & 1960 & 1962 & $\begin{array}{l}\text { arrived as Opuntia } \\
\text { leprocaulis }\end{array}$ \\
\hline Cylindropuntia ramosissima (Engelm.) F.M.Knuth & $\mathrm{nn}$ & $\begin{array}{l}\text { in collection } \\
\text { in } 1963\end{array}$ & 1982 & syn. Opuntia ramosissima \\
\hline Cylindropuntia rosea (DC.) Backeb. & Lokrum & 1967 & 1979 & from cuttings; 2 synonyms \\
\hline $\begin{array}{l}\text { Cylindropuntia whipplei (Engelm. \& J.M.Bigelow) } \\
\text { F.M.Knuth }\end{array}$ & Barcelona & 1963 & 1978 & syn. Opuntia whipplei \\
\hline \multirow[t]{2}{*}{ Denmoza rhodacantha (Salm-Dyck) Britton \& Rose } & nn & unknown & 1968 & $\begin{array}{l}\text { syn. Echinocactus } \\
\text { rhodacanthus }\end{array}$ \\
\hline & Bevedere & 1971 & 1980 & $\begin{array}{l}\text { arrived as E. erythrocepha- } \\
\text { la; one of } 8 \text { synonyms }\end{array}$ \\
\hline Disocactus biformis (Lindl.) Lindl. & Berlin & $1968 \& 1973$ & $1983 \& 1977$ & syn. Epiphyllum biforme \\
\hline \multirow[t]{3}{*}{ Disocactus flagelliformis (L.) Barthlott } & Besancon & 1955 & 1963 & $\begin{array}{l}\text { arrived as Cereus } \\
\text { flagelliformis; one of } 30+ \\
\text { synonyms }\end{array}$ \\
\hline & $\mathrm{nn}$ & 1951 & 1990 & \\
\hline & Iasi & 1963 & 1975 & \\
\hline Disocactus nelsonii (Britton \& Rose) Linding. & Nantes & 1953 & 1989 & $\begin{array}{l}\text { arrived as Chiapasia } \\
\text { nelsonii; one of } 8 \\
\text { synonyms; from cuttings }\end{array}$ \\
\hline \multirow[t]{2}{*}{ Disocactus phyllanthoides (DC.) Barthlott } & nn & 1951 & 1961 & $\begin{array}{l}\text { arrived as Phyllocactus } \\
\text { phyllanthoides; one of 10+ } \\
\text { synonyms; from cuttings }\end{array}$ \\
\hline & Antwerpen & $1968 \& 1973$ & $1989 \& 1983$ & \\
\hline \multirow[t]{12}{*}{ Echinocactus grusonii Hildm. } & Adelaide & $\begin{array}{l}1957 \& 1971 \\
\& 1973\end{array}$ & $\begin{array}{l}1975 \& 1982 \& \\
1980\end{array}$ & $\begin{array}{l}\text { arrived as Echinopsis } \\
\text { grusonii; } 4 \text { synonyms }\end{array}$ \\
\hline & Palermo & 1964 & 2000 & $\begin{array}{l}\text { arrived as Echinocactus } \\
\text { grusonii }\end{array}$ \\
\hline & Lokrum & $1968 \& 1997$ & $1972 \& 2000$ & from cuttings (1997) \\
\hline & nn & 1954 & 1989 & \\
\hline & Berlin & $\begin{array}{l}1955 \& 1966 \\
\& 1968 \& \\
1969\end{array}$ & $\begin{array}{l}\text { no data \& } 1972 \\
\& 1972 \text { \& } 1972\end{array}$ & \\
\hline & Barcelona & $1960 \& 1961$ & $1962 \& 1978$ & \\
\hline & Antibes & 1962 & 1978 & \\
\hline & Halle & 1973 & 1990 & \\
\hline & Amsterdam & 1976 & 1978 & \\
\hline & Goeteborg & 1976 & 1979 & \\
\hline & Blanes & 1987 & 1990 & \\
\hline & Valencia & 1993 & 1996 & \\
\hline \multirow[t]{4}{*}{ Echinocactus platyacanthus Link \& Otto } & Barcelona & 1961 & 1989 & $\begin{array}{l}\text { arrived as E. palmeri; one } \\
\text { of } 5+\text { synonyms }\end{array}$ \\
\hline & Berlin & 1981 & 1981 & arrived as E. platycanthus \\
\hline & Barcelona & $1967 \& 1971$ & $1983 \& 1978$ & arrived as E. viznaga \\
\hline & Halle & 1973 & 1978 & \\
\hline Echinocereus berlandieri (Engelm.) Haage & nn & $\begin{array}{l}\text { in collection } \\
\text { in } 1961\end{array}$ & 1990 & $\begin{array}{l}\text { arrived as E. blanckii; one } \\
\text { of } 2 \text { synonyms }\end{array}$ \\
\hline Echinocereus cinerascens (DC.) Lem. & nn & $\begin{array}{l}\text { in collection } \\
\text { in } 1961\end{array}$ & 1989 & syn. Cereus cinerascens \\
\hline Echinocereus coccineus Engelm. & Monaco & 2000 & 2003 & $20+$ synonyms \\
\hline Echinocereus enneacanthus Engelm. & Halle & 1966 \& 1967 & 1978 \& 1981 & $\begin{array}{l}\text { Syn. Cereus enneacanthus; } \\
\text { from cuttings }\end{array}$ \\
\hline $\begin{array}{l}\text { Echinocereus longisetus (Engelm.) Lem. subsp. } \\
\text { delaetii (Gürke) N.P. Taylor }\end{array}$ & Lokrum & $\begin{array}{l}1960 \& 1967 \\
\& 1970\end{array}$ & $\begin{array}{l}1968 \& 1980 \& \\
1979\end{array}$ & $\begin{array}{l}\text { arrived as E. delaetii; one } \\
\text { of } 2 \text { synonyms }\end{array}$ \\
\hline Echinocereus maritimus (M.E. Jones) K. Schum. & $\begin{array}{l}\text { San Marino } \\
\text { (California) }\end{array}$ & 1984 & 1990 & syn. Cereus maritimus \\
\hline
\end{tabular}


S22 Sandev, D. et al.: Plethora of plants - collections of the Botanical Garden, Faculty of Science, University of Zagreb (2)

\begin{tabular}{|c|c|c|c|c|}
\hline Scientific name & $\begin{array}{l}\text { City of plant } \\
\text { material origin }\end{array}$ & $\begin{array}{l}\text { Year of } \\
\text { ordering/ } \\
\text { purchasing }\end{array}$ & $\begin{array}{l}\text { Year when plant } \\
\text { was last recorded } \\
\text { in collection }\end{array}$ & Notes and remarks \\
\hline \multirow[t]{3}{*}{ Echinocereus pentalophus (DC.) Lem. } & nn & unknown & 1963 & $\begin{array}{l}\text { arrived as Echinocactus } \\
\text { procumbens; syn. Cereus } \\
\text { procumbens }\end{array}$ \\
\hline & Halle & 1956 \& 1957 & 1978 \& 1993 & $\begin{array}{l}\text { arrived as Cereus } \\
\text { pentalophus }\end{array}$ \\
\hline & nn & $\begin{array}{l}\text { in collection } \\
\text { in } 1961\end{array}$ & 1990 & $\begin{array}{l}\text { arrived as E. pentalophus } \\
\text { var. procumbens }\end{array}$ \\
\hline \multirow[t]{2}{*}{$\begin{array}{l}\text { Echinocereus pentalophus subsp. leonensis } \\
\text { (Mathsson) N.P. Taylor }\end{array}$} & nn & $\begin{array}{l}\text { in collection } \\
\text { in } 1963\end{array}$ & 1966 & $\begin{array}{l}\text { arrived as E. leonensis; one } \\
\text { of } 2 \text { synonyms }\end{array}$ \\
\hline & nn & $\begin{array}{l}\text { in collection } \\
\text { in } 1961\end{array}$ & 1989 & \\
\hline \multirow[t]{2}{*}{$\begin{array}{l}\text { Echinocereus pentalophus var. ehrenbergii (Pfeiff.) } \\
\text { Backeb. }\end{array}$} & Halle & $1961 \& 1966$ & $1993 \& 1968$ & $\begin{array}{l}4 \text { synonyms; from cuttings } \\
(1966)\end{array}$ \\
\hline & nn & $\begin{array}{l}\text { in collection } \\
\text { in } 1975\end{array}$ & 2000 & \\
\hline Echinocereus polyacanthus Engelm. & Berlin & $1966 \& 1970$ & $1983 \& 1982$ & 2 synonyms \\
\hline \multirow[t]{3}{*}{$\begin{array}{l}\text { Echinocereus } \times \text { roetteri (Engelm.) Engelm. ex } \\
\text { Rümpler }\end{array}$} & Halle & 1953 & no data & $\begin{array}{l}\text { arrived as Echinopsis } \\
\text { roetteri; two synonyms; } \\
\text { from cuttings }\end{array}$ \\
\hline & Palermo & 1958 & 1982 & \\
\hline & nn & 1966 & 1978 & \\
\hline \multirow[t]{2}{*}{$\begin{array}{l}\text { Echinocereus scheeri (Salm-Dyck) Scheer subsp. } \\
\text { scheeri }\end{array}$} & Rotterdam & 1969 & 1978 & $\begin{array}{l}\text { arrived as E. salm-dycki- } \\
\text { anus; one of } 2 \text { synonyms }\end{array}$ \\
\hline & nn & unknown & 1978 & \\
\hline Echinocereus triglochidiatus Engelm. & Nantes & 1999 & 2000 & 5 synonyms \\
\hline Echinocereus viereckii Werderm. & Halle & 1966 & 1989 & $\begin{array}{l}\text { syn. E. viereckii subsp. } \\
\text { huastecensis }\end{array}$ \\
\hline Echinocereus viridiflorus Engelm. & Lokrum & 1967 & 1978 & 3 synonyms; from cuttings \\
\hline \multirow[t]{2}{*}{ Echinofossulocactus gladiatus (Link \& Otto) Lawr. } & nn & unknown & 1974 & 2 synonyms \\
\hline & Sarvar & 1973 & 1990 & \\
\hline \multirow[t]{8}{*}{ Echinopsis ancistrophora Speg. } & Berlin & $1964 \& 1973$ & 1966 \& 2000 & $\begin{array}{l}\text { arrived as Echinopsis } \\
\text { polyancistra; one of } 15+ \\
\text { synonyms }\end{array}$ \\
\hline & Wageningen & $1972 \& 1980$ & $1983 \& 1983$ & \\
\hline & Debrecen & 1980 & 1983 & \\
\hline & Halle & $\begin{array}{l}1961 \& 1963 \\
\& 1966\end{array}$ & $\begin{array}{l}1975 \& 1968 \& \\
1968\end{array}$ & $\begin{array}{l}\text { arrived as Pseudolobivia } \\
\text { polyancistra }\end{array}$ \\
\hline & Linz & 1958 & 1963 & \\
\hline & Berlin & 1958 & 1971 & \\
\hline & Gent & $1964 \& 1965$ & $1968 \& 1968$ & \\
\hline & Karlsruhe & 1965 & 1977 & \\
\hline \multirow[t]{5}{*}{$\begin{array}{l}\text { Echinopsis ancistrophora subsp. arachnacantha } \\
\text { (Buining \& F. Ritter) Rausch }\end{array}$} & Gent & $1961 \& 1962$ & $1975 \& 1975$ & $\begin{array}{l}\text { arrived as E. kratochviliana; } \\
\text { one of } 3 \text { synonyms }\end{array}$ \\
\hline & nn & 1964 & 1965 & \\
\hline & Halle & 1966 & 1975 & \\
\hline & Saarbruecken & 1968 & 1975 & \\
\hline & Budapest & 1978 & 1982 & $\begin{array}{l}\text { arrived as Lobivia } \\
\text { arachnacantha }\end{array}$ \\
\hline \multirow[t]{3}{*}{$\begin{array}{l}\text { Echinopsis atacamensis (Phil.) Friedrich \& } \\
\text { G.D.Rowley subsp. pasacana (F.A.C. Weber ex } \\
\text { Rümpler) G. Navarro }\end{array}$} & Barcelona & 1980 & 1989 & $\begin{array}{l}\text { arrived as Helianthocereus } \\
\text { pasacana; one of } 5 \\
\text { synonyms }\end{array}$ \\
\hline & Berlin & 1987 & 1990 & $\begin{array}{l}\text { arrived as Trichocereus } \\
\text { atacamensis }\end{array}$ \\
\hline & Berlin & 1953 & 1966 & $\begin{array}{l}\text { arrived as Trichocereus } \\
\text { pasacana }\end{array}$ \\
\hline \multirow[t]{7}{*}{ Echinopsis aurea Britton \& Rose } & Halle & 1953 \& 1959 & $1961 \& 1966$ & $\begin{array}{l}\text { arrived as Lobivia aurea; } \\
\text { one of } 20+\text { synonyms }\end{array}$ \\
\hline & Tuebingen & 1957 & 1963 & \\
\hline & Erlangen & 1965 & 1976 & \\
\hline & Monaco & 1989 & 1990 & \\
\hline & Linz & 1963 & 1976 & \begin{tabular}{|l|} 
arrived as Lobivia \\
famatimensis var. leucomalla
\end{tabular} \\
\hline & Halle & $\begin{array}{l}1961 \& 1962 \\
\& 1967\end{array}$ & $\begin{array}{l}1971 \& 1971 \& \\
1976\end{array}$ & $\begin{array}{l}\text { arrived as Pseudolobivia } \\
\text { aurea }\end{array}$ \\
\hline & Linz & $1963 \& 1964$ & $1975 \& 1975$ & \\
\hline
\end{tabular}




\begin{tabular}{|c|c|c|c|c|}
\hline Scientific name & $\begin{array}{l}\text { City of plant } \\
\text { material origin }\end{array}$ & $\begin{array}{l}\text { Year of } \\
\text { ordering/ } \\
\text { purchasing }\end{array}$ & $\begin{array}{l}\text { Year when plant } \\
\text { was last recorded } \\
\text { in collection }\end{array}$ & Notes and remarks \\
\hline & Prague & 1989 & 1990 & \\
\hline & Linz & 1972 & 1979 & $\begin{array}{l}\text { arrived as Pseudolobivia } \\
\text { aurea var.fallax }\end{array}$ \\
\hline & Halle & $1959 \& 1965$ & 1966 \& 1993 & $\begin{array}{l}\text { arrived as Lobivia } \\
\text { cylindrica }\end{array}$ \\
\hline & nn & $\begin{array}{l}\text { in collection } \\
\text { in } 1968\end{array}$ & 1971 & \\
\hline & Berlin & 1979 & 2010 & \\
\hline & Linz & $2002 \& 2003$ & $2010 \& 2010$ & \\
\hline Echinopsis aurea subsp. dobeana (Dölz) Lodé & Linz & 1972 & no data & $\begin{array}{l}\text { arrived as Lobivia dobeana; } \\
\text { one of } 2 \text { synonyms }\end{array}$ \\
\hline Echinopsis blossfeldiana Robl. & Halle & 1966 & 1972 & $?$ \\
\hline \multirow{2}{*}{ Echinopsis bridgesii Salm-Dyck } & Hamburg & 1955 & 1989 & 2 synonyms \\
\hline & Berlin & 1968 & 1989 & \\
\hline \multirow[t]{5}{*}{$\begin{array}{l}\text { Echinopsis candicans (Gillies in Salm-Dyck.) D.R. } \\
\text { Hunt }\end{array}$} & Marburg & $1953 \& 1955$ & no data \& 1968 & $\begin{array}{l}\text { arrived as Trichocereus } \\
\text { candicans; one of } 10+ \\
\text { synonyms }\end{array}$ \\
\hline & Graz & 1957 & 1989 & \\
\hline & Adelaide & 1972 & 1989 & \\
\hline & Berlin & 1987 & 1990 & \\
\hline & Monaco & 2003 & 2006 & \\
\hline \multirow[t]{2}{*}{ Echinopsis chamaecereus H.Friedrich \& Glaetzle } & $\mathrm{nn}$ & $\begin{array}{l}\text { in collection } \\
\text { in } 1961\end{array}$ & 1978 & $\begin{array}{l}\text { arrived as Chamaecereus } \\
\text { silvestrii; one of } 4 \\
\text { synonyms }\end{array}$ \\
\hline & Rotterdam & 1969 & 1990 & \\
\hline \multirow[t]{2}{*}{$\begin{array}{l}\begin{array}{l}\text { Echinopsis chiloensis (Colla) Friedrich \& } \\
\text { G.D.Rowley }\end{array} \\
\end{array}$} & Lokrum & 1967 & 1989 & syn. Trichocereus chiloensis \\
\hline & Bonn & 1985 & 1990 & \\
\hline \multirow[t]{3}{*}{ Echinopsis chrysantha Werderm. } & Halle & 1955 & 1961 & $\begin{array}{l}\text { arrived as Lobivia } \\
\text { chrysantha; one of } 2 \\
\text { synonyms }\end{array}$ \\
\hline & Erlangen & 1965 & 1975 & \\
\hline & $\begin{array}{l}\text { Vienna-Belve- } \\
\text { dere }\end{array}$ & 1993 & 1993 & \\
\hline \multirow[t]{4}{*}{ Echinopsis cinnabarina (Hook.) Labour. } & Linz & 1972 & 1982 & $\begin{array}{l}\text { arrived as Lobivia } \\
\text { acanthoplegma; one of } 5 \\
\text { synonyms }\end{array}$ \\
\hline & Gent & 1964 & 1977 & $\begin{array}{l}\text { arrived as Lobivia } \\
\text { cinnabarina }\end{array}$ \\
\hline & $\begin{array}{l}\text { Vienna-Belve- } \\
\text { dere }\end{array}$ & 1971 & 1983 & \\
\hline & Linz & $1963 \& 1970$ & 1968 \& 1979 & $\begin{array}{l}\text { arrived as Lobivia } \\
\text { walterspielii }\end{array}$ \\
\hline Echinopsis coronata Cárdenas & Vienna & 1984 & 1993 & 2 synonyms \\
\hline $\begin{array}{l}\text { Echinopsis crassicaulis (R. Kiesling) H. Friedrich } \\
\text { \& Glaetzle }\end{array}$ & Linz & $\begin{array}{l}1967 \& 1972 \\
\& 1975 \& \\
1978\end{array}$ & $\begin{array}{l}1982 \& 1993 \& \\
1980 \& 1983\end{array}$ & $\begin{array}{l}\text { arrived as Helianthocereus } \\
\text { crassicaulis }\end{array}$ \\
\hline Echinopsis decaisneana (Lem.) Walp. & Halle & $1951 \& 1955$ & $1961 \& 1978$ & $\begin{array}{l}\text { syn. Echinonyctanthus } \\
\text { decaisnianus; from cuttings } \\
\text { (1951) }\end{array}$ \\
\hline \multirow[t]{5}{*}{ Echinopsis densispina Werderm. } & Vienna & $1963 \& 1985$ & $1975 \& 1985$ & $\begin{array}{l}\text { arrived as Lobivia } \\
\text { famatimensis var. } \\
\text { densispina; one of } 3 \\
\text { synonyms }\end{array}$ \\
\hline & Halle & 1966 & 1971 & from cuttings \\
\hline & Erlangen & 1965 & 1975 & $\begin{array}{l}\text { arrived as Lobivia } \\
\text { rebutioides }\end{array}$ \\
\hline & Lokrum & 1970 & 1983 & \\
\hline & Dresden & 1986 & 1993 & \\
\hline Echinopsis densispina Werderm. f. chlorogona & Karlsruhe & 1967 & 1982 & $\begin{array}{l}\text { arrived as Lobivia } \\
\text { rebutioides f.chlorogona }\end{array}$ \\
\hline Echinopsis densispina Werderm. f. sublimiflora & Halle & 1961 & 1971 & $\begin{array}{l}\text { arrived as Lobivia } \\
\text { rebutioides f.sublimiflora }\end{array}$ \\
\hline $\begin{array}{l}\text { Echinopsis deserticola (Werderm.) Friedrich \& G.D. } \\
\text { Rowley }\end{array}$ & Barcelona & 1980 & 1993 & syn. Trichocereus deserticola \\
\hline Echinopsis duvallii Wedd. & Palermo & 1955 & 1975 & \\
\hline
\end{tabular}


S24 Sandev, D. et al.: Plethora of plants - collections of the Botanical Garden, Faculty of Science, University of Zagreb (2)

\begin{tabular}{|c|c|c|c|c|}
\hline Scientific name & $\begin{array}{l}\text { City of plant } \\
\text { material origin }\end{array}$ & $\begin{array}{l}\text { Year of } \\
\text { ordering/ } \\
\text { purchasing }\end{array}$ & $\begin{array}{l}\text { Year when plant } \\
\text { was last recorded } \\
\text { in collection }\end{array}$ & Notes and remarks \\
\hline $\begin{array}{l}\text { Echinopsis eyriesii (Turpin) Pfeiff. \& Otto var. } \\
\text { triumphans Jacobi in Foerster }\end{array}$ & Halle & $1957 \& 1959$ & $1989 \& 1978$ & arrived as E. triumphans \\
\hline \multirow[t]{4}{*}{ Echinopsis famatimensis (Speg.) Werderm. } & Halle & $\begin{array}{l}1959 \& 1961 \\
\& 1965\end{array}$ & $\begin{array}{l}1977 \& 1961 \& \\
1979\end{array}$ & $\begin{array}{l}\text { arrived as Lobivia } \\
\text { famatimensis; one of } 3 \\
\text { synonyms }\end{array}$ \\
\hline & Berlin & $\begin{array}{l}1958 \& 1959 \\
\& 1973\end{array}$ & $\begin{array}{l}1966 \& 1978 \& \text { \& } \\
1982\end{array}$ & \\
\hline & nn & $\begin{array}{l}\text { in collection } \\
\text { in } 1963\end{array}$ & 1978 & \\
\hline & Bologna & 1971 & 1976 & \\
\hline \multirow[t]{2}{*}{ Echinopsis ferox (Britton \& Rose) Backeb. } & $\begin{array}{l}\text { Vienne-Belve- } \\
\text { dere }\end{array}$ & 1993 & 1993 & $\begin{array}{l}\text { arrived as Lobivia ferox; } \\
\text { one of } 5+\text { synonyms }\end{array}$ \\
\hline & $\begin{array}{l}\text { Vienna-Belve- } \\
\text { dere }\end{array}$ & 1971 & 1983 & $\begin{array}{l}\text { arrived as Pseudolobivia } \\
\text { potosina }\end{array}$ \\
\hline Echinopsis fischeri hort. & Palermo & 1969 & 1979 & \\
\hline $\begin{array}{l}\text { Echinopsis glauca (F.Ritter) Friedrich \& } \\
\text { G.D.Rowley }\end{array}$ & Barcelona & 1980 & 2000 & $\begin{array}{l}\text { arrived as Trichocereus } \\
\text { glaucus; one of } 4 \\
\text { synonyms }\end{array}$ \\
\hline \multirow[t]{3}{*}{ Echinopsis haematantha (Speg.) D.R. Hunt } & Gent & $1963 \& 1966$ & $1970 \& 1978$ & $\begin{array}{l}\text { arrived as Pseudolobivia } \\
\text { hamatacantha; } 2 \text { synonyms }\end{array}$ \\
\hline & Linz & $1962 \& 1965$ & $1982 \& 1980$ & \\
\hline & Berlin & $1973 \& 1979$ & $1982 \&$ no data & $\begin{array}{l}\text { arrived as Lobivia } \\
\text { haematantha }\end{array}$ \\
\hline Echinopsis hammerschmidii Cárdenas & Linz & $1964 \& 1969$ & $1990 \& 1990$ & \\
\hline \multirow[t]{8}{*}{ Echinopsis hertrichiana (Backeb.) D.R. Hunt } & Halle & $\begin{array}{l}1955 \& 1961 \\
\& 1964\end{array}$ & $\begin{array}{l}1961 \& 1966 \& \& \\
1980\end{array}$ & $\begin{array}{l}\text { arrived as Lobivia } \\
\text { hertrichiana; one of 5+ } \\
\text { synonyms }\end{array}$ \\
\hline & Berlin & $\begin{array}{l}1962 \& 1965 \\
\& 1969\end{array}$ & $\begin{array}{l}1982 \& 1978 \& \text { \& } \\
1968\end{array}$ & \\
\hline & Linz & $1964 \& 1965$ & $1971 \& 1983$ & \\
\hline & $\mathrm{nn}$ & 1965 & 1982 & \\
\hline & Halle & $1966 \& 1968$ & $1983 \& 1975$ & $\begin{array}{l}\text { arrived as Lobivia } \\
\text { huilcanota; from cuttings }\end{array}$ \\
\hline & Debrecen & 1980 & 1983 & arrived as Lobivia laui \\
\hline & Halle & 1965 & no data & \begin{tabular}{|l} 
arrived as Lobivia \\
allegraiana
\end{tabular} \\
\hline & Halle & 1962 & 1968 & $\begin{array}{l}\text { arrived as Lobivia } \\
\text { binghamiana }\end{array}$ \\
\hline \multirow[t]{6}{*}{$\begin{array}{l}\text { Echinopsis huascha (F.A.C. Weber) Friedrich \& } \\
\text { G.D. Rowley }\end{array}$} & Berlin & 1953 & 1983 & $\begin{array}{l}\text { arrived as Trichocereus } \\
\text { huascha }\end{array}$ \\
\hline & Berlin & 1959 & 1966 & $\begin{array}{l}\text { arrived as Helianthocereus } \\
\text { grandiflorus; one of } 15+ \\
\text { synonyms }\end{array}$ \\
\hline & $\begin{array}{l}\text { Vienna-Belve- } \\
\text { dere }\end{array}$ & $1953 \& 1971$ & $1983 \& 1983$ & $\begin{array}{l}\text { arrived as Helianthocereus } \\
\text { huascha }\end{array}$ \\
\hline & Berlin & 1953 & 1983 & $\begin{array}{l}\text { arrived as Trichocereus } \\
\text { andalgalensis; from } \\
\text { cuttings }\end{array}$ \\
\hline & Barcelona & 1964 & 2003 & $\begin{array}{l}\text { arrived as Helianthocereus } \\
\text { huascha var. auricolor }\end{array}$ \\
\hline & nn & unknown & 1968 & \\
\hline \multirow[t]{2}{*}{ Echinopsis huotii (Cels.) Labour. } & $\begin{array}{l}\text { Vienna-Belve- } \\
\text { dere }\end{array}$ & 1971 & 1975 & $\begin{array}{l}\text { arrived as E. cochabamben- } \\
\text { sis }\end{array}$ \\
\hline & Linz & $\begin{array}{l}1965 \& 1970 \\
\& 1971\end{array}$ & $\begin{array}{l}1981 \& 1978 \& \\
1982\end{array}$ & arrived as E. comarapana \\
\hline Echinopsis lagemannii D. Dietr. & Palermo & 1957 & 1993 & syn. E. lagemannii \\
\hline \multirow[t]{2}{*}{ Echinopsis lateritia Gürke } & Berlin & 1965 & 1983 & $\begin{array}{l}\text { arrived as Lobivia lateritia; } \\
\text { one of } 10+\text { synonyms }\end{array}$ \\
\hline & Jibou & 1989 & 2003 & \\
\hline \multirow[t]{3}{*}{ Echinopsis leucantha (Gillies ex Salm-Dyck) Walp. } & Halle & 1955 & 1961 & $\begin{array}{l}\text { syn. Echinocactus } \\
\text { leucanthus }\end{array}$ \\
\hline & nn & $\begin{array}{l}\text { in collection } \\
\text { in } 1963\end{array}$ & 1982 & \\
\hline & Bucharest & 1985 & 1993 & \\
\hline
\end{tabular}




\begin{tabular}{|c|c|c|c|c|}
\hline Scientific name & $\begin{array}{l}\text { City of plant } \\
\text { material origin }\end{array}$ & $\begin{array}{l}\text { Year of } \\
\text { ordering/ } \\
\text { purchasing }\end{array}$ & \begin{tabular}{|l|} 
Year when plant \\
was last recorded \\
in collection
\end{tabular} & Notes and remarks \\
\hline & Berlin & 1987 & 1990 & \\
\hline $\begin{array}{l}\text { Echinopsis litoralis (Johow) Friedrich \& G.D. } \\
\text { Rowley }\end{array}$ & Santiago & 1968 & 1993 & 2 synonyms \\
\hline \multirow[t]{8}{*}{ Echinopsis mamillosa Gürke } & Basel & 1957 & 1982 & arrived as E. kermesina \\
\hline & Tuebingen & 1963 & 1978 & \\
\hline & Halle & 1965 & 1989 & \\
\hline & Karlsruhe & 1971 & 1982 & \\
\hline & Vienna & 1956 & 1966 & arrived as E. mamillosa \\
\hline & Linz & 1976 & 1989 & \\
\hline & Berlin & 1979 & 1983 & \\
\hline & Wageningen & 1980 & 1983 & \\
\hline \multirow[t]{5}{*}{ Echinopsis marsoneri Werderm. } & Halle & $\begin{array}{l}1957 \& 1965 \\
\& 1967\end{array}$ & $\begin{array}{l}1978 \& 1981 \& \\
1980\end{array}$ & arrived as Lobivia jajoiana \\
\hline & nn & 1962 & 1982 & \\
\hline & Berlin & 1979 & 1983 & \\
\hline & Berlin & $\begin{array}{l}1959 \& 1962 \\
\& 1965\end{array}$ & $\begin{array}{l}1976 \& 1961 \& \\
1980\end{array}$ & $\begin{array}{l}\text { arrived as Lobivia } \\
\text { marsoneri }\end{array}$ \\
\hline & Halle & 1965 & 1983 & arrived as Lobivia rubescens \\
\hline \multirow[t]{3}{*}{ Echinopsis maximiliana Heyder ex A. Dietr. } & Berlin & 1955 & 1983 & $\begin{array}{l}\text { arrived as Lobivia } \\
\text { caespitosa; one of } 3 \\
\text { synonyms }\end{array}$ \\
\hline & Graz & 1975 & 1989 & \\
\hline & Berlin & $1959 \& 1962$ & $1980 \& 1971$ & $\begin{array}{l}\text { arrived as Lobivia } \\
\text { hermanniana }\end{array}$ \\
\hline \multirow[t]{4}{*}{ Echinopsis mirabilis Speg. } & Wageningen & 1972 & 1979 & 4 synonyms \\
\hline & Nantes & 1970 & 1978 & \\
\hline & Prague & 1989 & 1993 & \\
\hline & Innsbruck & 2000 & 2006 & \\
\hline \multirow[t]{3}{*}{ Echinopsis multiplex (Pfeiff.) Zucc. } & nn & $\begin{array}{l}1952 \text { \& } 1952 \\
\& \text { in } \\
\text { collection in } \\
1963\end{array}$ & $\begin{array}{l}\text { no data \& } 1961 \\
\& 1972\end{array}$ & $\begin{array}{l}2 \text { synonyms; from cuttings } \\
\text { (1952) }\end{array}$ \\
\hline & Genova & 1962 & 1990 & \\
\hline & Wageningen & $1972 \& 1986$ & $1990 \& 1990$ & \\
\hline Echinopsis multiplex "var. rosea hort." & Berlin & 1973 & 1977 & unknown var. \\
\hline \multirow[t]{4}{*}{ Echinopsis obrepanda (Salm-Dyck) K. Schum. } & $\operatorname{Linz}$ & 1965 & 1982 & $\begin{array}{l}\text { arrived as E. rojasii; one of } \\
20+\text { synonyms }\end{array}$ \\
\hline & Linz & $1971 \& 1972$ & $1980 \& 1982$ & arrived as E. toralapana \\
\hline & Halle & $\begin{array}{l}1959 \& 1961 \\
\& 1962 \& \\
1965\end{array}$ & $\begin{array}{l}1989 \& 1968 \& \\
1975 \& 1982\end{array}$ & $\begin{array}{l}\text { arrived as Pseudolobivia } \\
\text { obrepanda }\end{array}$ \\
\hline & Jibou & 1994 & $1983-2000$ & \\
\hline \multirow[t]{3}{*}{ Echinopsis oxygona (Link) Zucc. } & Berlin & 1979 & 1990 & $\begin{array}{l}\text { arrived as E. eyriesii; one } \\
\text { of } 3 \text { synonyms }\end{array}$ \\
\hline & Halle & $1957 \& 1958$ & $1990 \& 1990$ & arrived as E. paraguayensis \\
\hline & Muenchen & 1959 & 1963 & \\
\hline Echinopsis pampana (Britton \& Rose) D.R. Hunt & Berlin & $1964 \& 1965$ & 1978 \& 1977 & $\begin{array}{l}\text { arrived as Lobivia } \\
\text { mistiensis; one of } 3 \\
\text { synonyms }\end{array}$ \\
\hline Echinopsis pelecyrhachis Backeb. & Berlin & 1979 & 1983 & $\begin{array}{l}\text { syn. Pseudolobivia } \\
\text { pelecyrhachis }\end{array}$ \\
\hline \multirow[t]{8}{*}{$\begin{array}{l}\text { Echinopsis pentlandii (Hook.) Salm-Dyck ex A. } \\
\text { Dietr. }\end{array}$} & Muenchen & 1972 & 1983 & $\begin{array}{l}\text { arrived as Lobivia } \\
\text { higginsiana; one of } 7 \\
\text { synonyms }\end{array}$ \\
\hline & Debrecen & 1980 & 1983 & arrived as Lobivia larae \\
\hline & Berlin & 1960 & 1977 & $\begin{array}{l}\text { arrived as Lobivia } \\
\text { leucorhodon }\end{array}$ \\
\hline & Sarvar & 1973 & 1980 & \\
\hline & Jibou & 1989 & 1993 & \\
\hline & Liege & 1985 & 1989 & $\begin{array}{l}\text { arrived as Lobivia } \\
\text { multicostata }\end{array}$ \\
\hline & Halle & $1961 \& 1965$ & $1979 \& 1983$ & $\begin{array}{l}\text { arrived as Lobivia } \\
\text { schneideriana }\end{array}$ \\
\hline & Linz & 1963 & 1982 & \\
\hline
\end{tabular}


S26 Sandev, D. et al.: Plethora of plants - collections of the Botanical Garden, Faculty of Science, University of Zagreb (2)

\begin{tabular}{|c|c|c|c|c|}
\hline Scientific name & $\begin{array}{l}\text { City of plant } \\
\text { material origin }\end{array}$ & $\begin{array}{l}\text { Year of } \\
\text { ordering/ } \\
\text { purchasing }\end{array}$ & $\begin{array}{l}\text { Year when plant } \\
\text { was last recorded } \\
\text { in collection }\end{array}$ & Notes and remarks \\
\hline & Berlin & 1965 & 1983 & arrived as Lobivia varians \\
\hline & Debrecen & 1980 & 1983 & arrived as Lobivia argentea \\
\hline $\begin{array}{l}\text { Echinopsis pentlandii var.forbesii (A.Dietr.) Rud. } \\
\text { Mey. }\end{array}$ & Gent & 1967 & 1980 & syn. Lobivia pentlandii \\
\hline \multirow[t]{2}{*}{ Echinopsis rhodotricha K. Schum. } & Halle & 1957 & 1983 & $\begin{array}{l}\text { syn. Acanthocalycium } \\
\text { rhodotrichum }\end{array}$ \\
\hline & nn & $\begin{array}{l}\text { in collection } \\
\text { in } 1975\end{array}$ & 1990 & \\
\hline Echinopsis $\times$ rohlandii K.Schum. & Berlin & 1953 & no data & arrived as E. rohlandii hort. \\
\hline Echinopsis sanguiniflora (Backeb.) D.R. Hunt & Halle & $1965 \& 1966$ & $1975 \& 1978$ & $\begin{array}{l}\text { arrived as Lobivia } \\
\text { sanguiniflora }\end{array}$ \\
\hline Echinopsis schickendantzii F.A.C. Weber & Barcelona & 1971 & 1990 & $\begin{array}{l}\text { arrived as Trichocereus } \\
\text { schickendantzii; one of } 4 \\
\text { synonyms }\end{array}$ \\
\hline Echinopsis schieliana (Backeb.) D.R. Hunt & Karlsruhe & $1963 \& 1965$ & $1968 \& 1978$ & $\begin{array}{l}\text { arrived as Lobivia } \\
\text { schieliana; one of } 2 \\
\text { synonyms }\end{array}$ \\
\hline \multirow[t]{2}{*}{$\begin{array}{l}\text { Echinopsis schreiteri (A. Cast.) Werderm. ex } \\
\text { Backeb. }\end{array}$} & Halle & $1965 \& 1967$ & $1989 \& 1990$ & arrived as Lobivia schreiteri \\
\hline & Berlin & 1973 & 1983 & \\
\hline \multirow[t]{3}{*}{ Echinopsis silvestrii Speg. } & nn & 1952 & no data & from cuttings \\
\hline & Palermo & $1957 \& 1960$ & $1978 \& 1978$ & \\
\hline & Linz & 1972 & 1979 & \\
\hline \multirow[t]{2}{*}{$\begin{array}{l}\text { Echinopsis tarijensis (Vaupel) Friedrich \& G.D. } \\
\text { Rowley }\end{array}$} & Berlin & $1985 \& 1986$ & $1989 \& 1990$ & $\begin{array}{l}\text { arrived as Helianthocereus } \\
\text { tarijensis; one of } 10+ \\
\text { synonyms }\end{array}$ \\
\hline & Barcelona & 1980 & 1989 & $\begin{array}{l}\text { arrived as Trichocereus } \\
\text { narvaecensis }\end{array}$ \\
\hline \multirow[t]{5}{*}{$\begin{array}{l}\text { Echinopsis terscheckii (Parm. ex Pfeiff.) Friedrich \& } \\
\text { G.D. Rowley }\end{array}$} & Halle & 1966 & 1966 & $\begin{array}{l}\text { arrived as Trichocereus } \\
\text { terscheckii; one of } 15+ \\
\text { synonyms; from cuttings }\end{array}$ \\
\hline & Muenchen & 1960 & 1968 & \\
\hline & Berlin & 1966 & 1989 & \\
\hline & Adelaide & 1972 & 1977 & $\begin{array}{l}\text { arrived as Trichocereus } \\
\text { werdermannianus }\end{array}$ \\
\hline & Barcelona & 1980 & 1990 & \\
\hline \multirow[t]{3}{*}{$\begin{array}{l}\text { Echinopsis thelegonoides (Speg.) H. Friedrich \& } \\
\text { G.D. Rowley }\end{array}$} & Barcelona & 1980 & 1989 & $\begin{array}{l}\text { arrived as Trichocereus } \\
\text { thelegonoides; one of } 4 \\
\text { synonyms }\end{array}$ \\
\hline & Berlin & $\begin{array}{l}1961 \& 1963 \\
\& 1967 \& \\
2000\end{array}$ & $\begin{array}{l}1989 \& 2000 \& \\
2000 \& 2003\end{array}$ & \\
\hline & Berlin & $\begin{array}{l}\text { in collection } \\
\text { in } 1968\end{array}$ & 1978 & \\
\hline Echinopsis tiegeliana (Wessner) D.R. Hunt & Halle & $1961 \& 1963$ & $1978 \& 1979$ & $\begin{array}{l}\text { arrived as Lobivia } \\
\text { peclardiana; one of } 2 \\
\text { synonyms }\end{array}$ \\
\hline \multirow[t]{4}{*}{ Echinopsis tubiflora (Pfeiff.) Zucc. ex A. Dietr. } & nn & 1951 & 1989 & 3 synonyms \\
\hline & Berlin & $1953 \& 1979$ & $1963 \& 2000$ & \\
\hline & Halle & $1951 \& 1959$ & $1977 \& 1975$ & \\
\hline & Wageningen & 1972 & 1983 & \\
\hline \multirow[t]{2}{*}{ Echinopsis turbinata Zucc. } & Berlin & 1962 & 1989 & \\
\hline & nn & $\begin{array}{l}\text { in collection } \\
\text { in } 1968\end{array}$ & 1980 & \\
\hline \multirow[t]{2}{*}{$\begin{array}{l}\text { Echinopsis uyupampensis (Backeb.) Friedrich \& } \\
\text { G.D. Rowley }\end{array}$} & Lokrum & $\begin{array}{l}1968 \& 1969 \\
\& 1970\end{array}$ & $\begin{array}{l}1978 \& 1983 \& \\
2003\end{array}$ & $\begin{array}{l}\text { arrived as Trichocereus } \\
\text { validus }\end{array}$ \\
\hline & Besancon & 1969 & 1976 & \\
\hline \multirow[t]{3}{*}{ Epiphyllum anguliger (Lem.) G. Don } & Berlin & $1968 \& 1971$ & $1993 \& 1989$ & 2 synonyms \\
\hline & Gent & 1970 & 1979 & \\
\hline & Berlin & 1967 & 1984 & arrived as E. darrahii \\
\hline Epiphyllum crenatum (Lindl.) G.Don & $\mathrm{nn}$ & unknown & 1978 & 2 synonyms \\
\hline Epiphyllum oxypetalum (DC.) Haw. & Antwerpen & $\begin{array}{l}1967 \& 1969 \\
\& 1970 \& \\
1973\end{array}$ & $\begin{array}{l}1971 \& 1978 \& \\
1979 \& 1983\end{array}$ & $\begin{array}{l}\text { arrived Phyllocactus } \\
\text { latifrons; one od } 10+ \\
\text { synonyms }\end{array}$ \\
\hline
\end{tabular}




\begin{tabular}{|c|c|c|c|c|}
\hline Scientific name & $\begin{array}{l}\text { City of plant } \\
\text { material origin }\end{array}$ & $\begin{array}{l}\text { Year of } \\
\text { ordering/ } \\
\text { purchasing }\end{array}$ & $\begin{array}{l}\text { Year when plant } \\
\text { was last recorded } \\
\text { in collection }\end{array}$ & Notes and remarks \\
\hline \multirow[t]{2}{*}{ Epiphyllum phyllanthus (L.) Haw. } & Berlin & 1966 & 1984 & $10+$ synonyms \\
\hline & Munchen & $1970 \& 1977$ & $1984 \& 1984$ & \\
\hline \multirow[t]{2}{*}{$\begin{array}{l}\text { Epithelantha micromeris (Engelm.) F.A.C. Weber ex } \\
\text { Britton \& Rose }\end{array}$} & nn & $\begin{array}{l}\text { in collection } \\
\text { in } 1963\end{array}$ & 1983 & $\begin{array}{l}\text { arrived as Lophophora } \\
\text { lewinii; one of } 5+ \\
\text { synonyms }\end{array}$ \\
\hline & Vienna & 1964 & 1964 & $\begin{array}{l}\text { arrived as Mammillaria } \\
\text { micromeris; from cuttings }\end{array}$ \\
\hline \multirow[t]{2}{*}{ Eriosyce chilensis (Hildm. ex K. Schum.) Katt. } & Muenchen & 1977 & 1983 & $\begin{array}{l}\text { arrived as Neochilenia } \\
\text { chilensis; one of } 5+ \\
\text { synonyms }\end{array}$ \\
\hline & Linz & $1965 \& 1967$ & 1983 \& 1983 & $\begin{array}{l}\text { arrived as Neochilenia } \\
\text { chilensis var. albidiflora }\end{array}$ \\
\hline Eriosyce curvispina (Bertero ex Colla) Katt. & Lokrum & 1969 & 1970 & $\begin{array}{l}\text { arrived as Horridocactus } \\
\text { tuberisulcatus }\end{array}$ \\
\hline Eriosyce kunzei (Foerster) Katt. & Berlin & 1960 & 1966 & $\begin{array}{l}\text { arrived as Echinocactus } \\
\text { geissei; one of } 2 \text { synonyms }\end{array}$ \\
\hline Eriosyce limariensis (F. Ritter) Katt. & Palermo & 1957 & 1993 & $\begin{array}{l}\text { arrived as Horridocactus } \\
\text { nigricans; one of } 10+ \\
\text { synonyms }\end{array}$ \\
\hline \multirow[t]{2}{*}{ Eriosyce napina (Phil.) Katt. } & Muenchen & 1977 & 1979 & $\begin{array}{l}\text { arrived as Neochilenia } \\
\text { napina; one of } 5+ \\
\text { synonyms }\end{array}$ \\
\hline & Goettingen & unknown & 1993 & \\
\hline $\begin{array}{l}\text { Eriosyce odieri (Lem. ex Salm-Dyck) Katt. subsp. } \\
\text { odieri }\end{array}$ & Wageningen & 1980 & 1983 & $\begin{array}{l}\text { arrived as Neochilenia } \\
\text { odieri; one of } 5 \text { synonyms }\end{array}$ \\
\hline Eriosyce recondita (F. Ritter) Katt. & Berlin & 1965 & 1965 & $\begin{array}{l}\text { arrived as Neochilenia } \\
\text { recondita; one of } 3 \\
\text { synonyms }\end{array}$ \\
\hline Eriosyce subgibbosa (Haw.) Katt. & Santiago & 1968 & 1977 & $\begin{array}{l}\text { arrived as Neoporteria } \\
\text { subgibbosa; one of } 4 \\
\text { synonyms }\end{array}$ \\
\hline \multirow[t]{4}{*}{$\begin{array}{l}\text { Eriosyce taltalensis (Hutchison) Katt. subsp. } \\
\text { paucicostata (F. Ritter) Katt. }\end{array}$} & Vienna & 1965 & 1983 & $\begin{array}{l}\text { arrived as Neoporteria } \\
\text { fusca; one of } 5 \text { synonyms }\end{array}$ \\
\hline & Linz & 1963 & 1977 & $\begin{array}{l}\text { arrived as Neochilenia } \\
\text { hankeana }\end{array}$ \\
\hline & Sarvar & 1973 & 1983 & \\
\hline & nn & 1966 & 1975 & $\begin{array}{l}\text { arrived as Neochilenia } \\
\text { paucicostata }\end{array}$ \\
\hline $\begin{array}{l}\text { Escobaria dasyacantha (Engelm.) Britton \& Rose } \\
\text { subsp. chaffeyi (Britton \& Rose) N.P. Taylor }\end{array}$ & Karlsruhe & 1965 & 1968 & $\begin{array}{l}\text { arrived as E. chaffeyi; one } \\
\text { of } 2 \text { synonyms }\end{array}$ \\
\hline \multirow[t]{2}{*}{ Escobaria tuberculosa (Engelm.) Britton \& Rose; } & Dresden & 2000 & 2010 & $5+$ synonyms \\
\hline & Monaco & 2003 & 2010 & \\
\hline Escobaria vivipara (Nutt.) Buxb. & Alberta & 1962 & 1968 & $\begin{array}{l}\text { arrived as Mammillaria } \\
\text { vivipara; one of } 5 \\
\text { synonyms }\end{array}$ \\
\hline Espostoa $\times$ cleistocactus & Barcelona & 1980 & 1989 & \\
\hline \multirow[t]{5}{*}{ Espostoa lanata (Kunth) Britton \& Rose } & Halle & 1973 & 1978 & $\begin{array}{l}\text { arrived as E. laticornua } \\
\text { var. atroviolacea; one of } 30+ \\
\text { synonyms }\end{array}$ \\
\hline & Monaco & 1997 & 2000 & arrived as E. lanata \\
\hline & nn & 1962 & 1979 & \\
\hline & Beograd & 1965 & 1968 & \\
\hline & Barcelona & $\begin{array}{l}1967 \& 1968 \\
\& 1984 \& \\
1993\end{array}$ & $\begin{array}{l}1975 \& 1993 \& \\
1990 \& 1993\end{array}$ & \\
\hline Espostoa melanostele (Vaupel) Borg & Lokrum & 1969 & 1971 & $\begin{array}{l}\text { arrived as Pseudoespostoa } \\
\text { melanostele }\end{array}$ \\
\hline \multirow[t]{2}{*}{ Espostoa ritteri Buining } & Barcelona & 1980 & 2000 & \\
\hline & Monaco & 1997 & 2000 & \\
\hline \multirow[t]{2}{*}{ Espostoopsis dybowskii (Rol.-Goss.) Buxb. } & Muenster & 1968 & 1983 & $\begin{array}{l}\text { arrived as Austrocephaloce- } \\
\text { reus dybowskii; one of } 5 \\
\text { synonyms }\end{array}$ \\
\hline & Amsterdam & 1968 & 1981 & \\
\hline Eulychnia breviflora Phil. & Liege & 1973 & 1977 & arrived as E. longispina \\
\hline
\end{tabular}


S28 Sandev, D. et al.: Plethora of plants - collections of the Botanical Garden, Faculty of Science, University of Zagreb (2)

\begin{tabular}{|c|c|c|c|c|}
\hline Scientific name & $\begin{array}{l}\text { City of plant } \\
\text { material origin }\end{array}$ & $\begin{array}{l}\text { Year of } \\
\text { ordering/ } \\
\text { purchasing }\end{array}$ & $\begin{array}{l}\text { Year when plant } \\
\text { was last recorded } \\
\text { in collection }\end{array}$ & Notes and remarks \\
\hline & Liege & 1973 & 1977 & arrived as E. saint-pieana \\
\hline Eulychnia procumbens Backeb. & Liege & 1973 & 1977 & \\
\hline \multirow[t]{5}{*}{ Ferocactus glaucescens (DC.) Britton \& Rose } & Palermo & $1957 \& 1962$ & $1980 \& 1980$ & $\begin{array}{l}\text { arrived as Echinocactus } \\
\text { pfeifferi }\end{array}$ \\
\hline & nn & $\begin{array}{l}\text { in collection } \\
\text { in } 1968\end{array}$ & 1980 & \\
\hline & Muenchen & 1961 & 1983 & $\begin{array}{l}\text { arrived as F. glaucescens } \\
\text { var. pfeifferi }\end{array}$ \\
\hline & Halle & 1966 & 1966 & \\
\hline & nn & $\begin{array}{l}\text { in collection } \\
\text { in } 1983\end{array}$ & 2000 & \\
\hline \multirow[t]{2}{*}{$\begin{array}{l}\text { Ferocactus hamatacanthus (Muehlenpf.) Britton } \\
\text { \& Rose }\end{array}$} & Barcelona & 1964 & 1968 & $\begin{array}{l}\text { arrived as Hamatocactus } \\
\text { hamatacanthus; one of } 4 \\
\text { synonyms }\end{array}$ \\
\hline & Goettingen & 1971 & 1978 & \\
\hline \multirow[t]{5}{*}{ Ferocactus wislizeni (Engelm.) Britton \& Rose } & Halle & 1966 & 1968 & $15+$ synonyms \\
\hline & Lokrum & 1969 & 1971 & \\
\hline & Liege & 1973 & 1975 & \\
\hline & Monaco & $1989 \& 1994$ & $1993 \& 2000$ & \\
\hline & Barcelona & 1994 & 2016 & \\
\hline \multirow[t]{2}{*}{ Frailea pumila (Lem.) Britton \& Rose } & Liege & 1984 & 2000 & $\begin{array}{l}\text { arrived as F. carminifila- } \\
\text { mentosa; one of } 2 \\
\text { synonyms }\end{array}$ \\
\hline & Graz & 1997 & 2000 & \\
\hline \multirow[t]{2}{*}{$\begin{array}{l}\text { Gymnocalycium anisitsii (K. Schum.) Britton \& } \\
\text { Rose }\end{array}$} & Linz & $1965 \& 1967$ & $1979 \& 1983$ & $\begin{array}{l}\text { arrived as G. damsii var. } \\
\text { Torulosum }\end{array}$ \\
\hline & Linz & 1968 & 1975 & syn. Echinocactus anisitsii \\
\hline \multirow[t]{2}{*}{ Gymnocalycium baldianum (Speg.) Speg. } & Berlin & $\begin{array}{l}1953 \& 1955 \\
\& 1967 \& \\
1968\end{array}$ & $\begin{array}{l}1961 \& 1983 \& \& \\
1983 \& 1983\end{array}$ & $\begin{array}{l}3 \text { synonyms; from cuttings } \\
(1953)\end{array}$ \\
\hline & Linz & $\begin{array}{l}1966 \& 1972 \\
\& 1975 \& \\
1976 \& 1978\end{array}$ & $\begin{array}{l}1981 \& 1983 \& \\
1982 \& 1983 \& \\
1982\end{array}$ & \\
\hline $\begin{array}{l}\text { Gymnocalycium bodenbenderianum (Hosseus ex } \\
\text { A.Berger) A.Berger subsp. intertextum (Backeb. ex } \\
\text { H. Till) H. Till }\end{array}$ & Linz & 1975 & 1982 & $\begin{array}{l}\text { arrived as G. moserianum; } \\
\text { syn. G. intertextum }\end{array}$ \\
\hline \multirow[t]{4}{*}{ Gymnocalycium bruchii (Speg.) Hosseus } & Tuebingen & $1963 \& 1964$ & $1983 \& 1980$ & 3 synonyms \\
\hline & Karlsruhe & 1965 & 1982 & \\
\hline & Muenchen & 1975 & 1983 & \\
\hline & Goettingen & 1985 & 1990 & \\
\hline Gymnocalycium capillaense (Schick) Hosseus & Berlin & $\begin{array}{l}1958 \& 1962 \\
\& 1965 \& \\
1968\end{array}$ & $\begin{array}{l}1966 \& 1966 \& \\
1973 \& 1980\end{array}$ & $\begin{array}{l}\text { arrived as G. sigelianum; } \\
\text { one of } 2 \text { synonyms }\end{array}$ \\
\hline \multirow[t]{5}{*}{$\begin{array}{l}\text { Gymnocalycium denudatum (Link \& Otto) Pfeiff. } \\
\text { ex Mittler }\end{array}$} & Berlin & 1955 \& 1979 & $1983 \& 1983$ & 9 synonyms \\
\hline & Halle & 1955 & 1971 & \\
\hline & Meinz & 1966 & 1976 & \\
\hline & Frankfurt & 1992 & 1993 & \\
\hline & Dresden & 1998 & 2000 & \\
\hline Gymnocalycium friedrichii (Werderm.) Pazout & Muenchen & 1975 & 1980 & $\begin{array}{l}\text { arrived as G. mihanovichii } \\
\text { var. friedrichii; one of } 2 \\
\text { synonyms }\end{array}$ \\
\hline \multirow[t]{9}{*}{ Gymnocalycium gibbosum (Haw.) Pfeiff. ex Mittler } & Hamburg & 1957 & 1978 & $\begin{array}{l}\text { arrived as G. chubutense; } \\
\text { one of } 20+\text { synonyms }\end{array}$ \\
\hline & Nantes & 1973 & 1975 & $\begin{array}{l}\text { arrived as G. gibbosum var. } \\
\text { gerardii }\end{array}$ \\
\hline & Erlangen & 1965 & 1982 & arrived as G. leeanum \\
\hline & Berlin & $1955 \& 1959$ & $1983 \& 1978$ & arrived as G. gibbosum \\
\hline & Halle & 1964 & 1979 & \\
\hline & Muenster & 1968 & 1983 & \\
\hline & Linz & 1978 & 1982 & \\
\hline & Goettingen & 1985 & 1993 & \\
\hline & Dresden & 1998 & 2000 & \\
\hline \multirow[t]{2}{*}{ Gymnocalycium gibbosum subsp. ferox Papsch } & Berlin & 1959 & 1968 & \\
\hline & Linz & 1965 & 1982 & \\
\hline
\end{tabular}




\begin{tabular}{|c|c|c|c|c|}
\hline Scientific name & $\begin{array}{l}\text { City of plant } \\
\text { material origin }\end{array}$ & $\begin{array}{l}\text { Year of } \\
\text { ordering/ } \\
\text { purchasing }\end{array}$ & $\begin{array}{l}\text { Year when plant } \\
\text { was last recorded } \\
\text { in collection }\end{array}$ & Notes and remarks \\
\hline \multirow[t]{3}{*}{ Gymnocalycium hossei (F.Haage) A.Berger } & Berlin & 1958 & 1978 & 3 synonyms \\
\hline & Linz & 1972 & 1975 & arrived as G. mazanense \\
\hline & Muenchen & 1972 & 1975 & \\
\hline Gymnocalycium hybopleurum (K.Schum.) Backeb. & Linz & $1965 \& 1978$ & 1980 \& no data & $\begin{array}{l}\text { syn. Echinocactus } \\
\text { multiflorus var. hybopleurus }\end{array}$ \\
\hline $\begin{array}{l}\text { Gymnocalycium leeanum (Hook.) Britton \& Rose } \\
\text { var. netrelianum (Monv.) Backeb. }\end{array}$ & Berlin & 1973 & 1978 & 2 synonyms \\
\hline \multirow[t]{2}{*}{ Gymnocalycium marsoneri Frič ex Y.Itô } & Linz & 1971 & 1978 & 2 synonyms \\
\hline & $\begin{array}{l}\text { Vienna-Belve- } \\
\text { dere }\end{array}$ & 1971 & 1980 & \\
\hline $\begin{array}{l}\text { Gymnocalycium megalothelos (Sencke) Britton \& } \\
\text { Rose }\end{array}$ & Berlin & 1979 & 1983 & 2 synonyms \\
\hline $\begin{array}{l}\text { Gymnocalycium mihanovichii (Frič ex Gürke) } \\
\text { Britton \& Rose }\end{array}$ & nn & 1967 & 1971 & $\begin{array}{l}\text { syn. Echinocactus } \\
\text { mihanovichii; gift from } \\
\text { Cvitanović }\end{array}$ \\
\hline \multirow[t]{3}{*}{$\begin{array}{l}\text { Gymnocalycium monvillei (Lem.) Pfeiff. ex Britton } \\
\& \text { Rose }\end{array}$} & Halle & 1965 & 1975 & syn. Echinocactus monvillei \\
\hline & Muenchen & 1975 & 1983 & \\
\hline & Jibou & 1989 & 1993 & \\
\hline \multirow[t]{2}{*}{ Gymnocalycium mostii (Gürke) Britton \& Rose } & Halle & 1961 & 1962 & $\begin{array}{l}\text { arrived as G. mostii var. } \\
\text { kurtzianum; one of } 2 \\
\text { synonyms }\end{array}$ \\
\hline & Linz & 1972 & 1982 & arrived as G. mostii \\
\hline Gymnocalycium ochoterenae Backeb. & Linz & 1967 & 1982 & \\
\hline $\begin{array}{l}\text { Gymnocalycium ochoterenae subsp. vatteri } \\
\text { (Buining) Papsch }\end{array}$ & Linz & 1968 \& 1971 & $1982 \& 1982$ & $\begin{array}{l}\text { arrived as G. ochoterenae } \\
\text { var. variispinum }\end{array}$ \\
\hline Gymnocalycium paroulum (Speg.) Speg. & Vienna & 1967 & 1980 & $\begin{array}{l}\text { syn. Echinocactus platensis } \\
\text { var. paroulus }\end{array}$ \\
\hline Gymnocalycium pflanzii (Vaupel) Werderm. & Linz & 1961 & 1983 & $\begin{array}{l}\text { arrived as G. lagunil- } \\
\text { lasense; one of } 2 \text { synonyms }\end{array}$ \\
\hline Gymnocalycium platense (Speg.) Britton \& Rose & Erlangen & 1965 & 1978 & 2 synonyms \\
\hline \multirow[t]{3}{*}{$\begin{array}{l}\text { Gymnocalycium quehlianum (F.Haage ex Quehl) } \\
\text { Vaupel ex Hosseus }\end{array}$} & Sankt Peterburg & 1961 & 1979 & $5+$ synonyms \\
\hline & Linz & $1987 \& 1991$ & $1990 \& 2010$ & \\
\hline & Monaco & 2003 & 2010 & \\
\hline \multirow[t]{4}{*}{ Gymnocalycium saglionis (Cels) Britton \& Rose } & Berlin & 1953 & 1961 & from cuttings; 3 synonyms \\
\hline & Halle & 1965 & 1983 & \\
\hline & Goettingen & 1985 & 1990 & \\
\hline & Linz & 1973 & 1974 & \\
\hline $\begin{array}{l}\text { Gymnocalycium saglionis subsp. tilcarense (Backeb.) } \\
\text { H.Till \& W.Till }\end{array}$ & Berlin & 1987 & 1990 & $\begin{array}{l}\text { arrived as G. saglionis var. } \\
\text { tilcarense; } 3 \text { synonyms }\end{array}$ \\
\hline \multirow[t]{2}{*}{ Gymnocalycium stellatum Speg. } & Vienna & 1967 & 1983 & $\begin{array}{l}\text { arrived as G. asterium; one } \\
\text { of } 2 \text { synonyms }\end{array}$ \\
\hline & Karlsruhe & 1965 & 1975 & arrived as G. stellatum \\
\hline Gymnocalycium uebelmannianum Rausch & Linz & 1973 & 1981 & \\
\hline \multirow[t]{3}{*}{$\begin{array}{l}\text { Gymnocalycium uruguayense (Arechav.) Britton } \\
\text { \& Rose }\end{array}$} & Linz & 1975 & 1982 & $\begin{array}{l}\text { arrived as G. artigas; one } \\
\text { of } 5+\text { synonyms }\end{array}$ \\
\hline & Hamburg & $1957 \& 1967$ & $1982 \& 1968$ & arrived as G. guerkeanum \\
\hline & Linz & $\begin{array}{l}1971 \& 1972 \\
\& 1973 \& \\
1987\end{array}$ & $\begin{array}{l}1978 \& 1982 \& \\
1982 \& 1993\end{array}$ & \\
\hline \multirow[t]{2}{*}{ Haageocereus acranthus (Vaupel) Backeb. } & $\begin{array}{l}\text { Vienna-Belve- } \\
\text { dere }\end{array}$ & 1971 & 1974 & 4 synonyms \\
\hline & Barcelona & $1971 \& 1972$ & $1990 \& 1983$ & \\
\hline $\begin{array}{l}\text { Haageocereus acranthus subsp. zonatus (Rauh \& } \\
\text { Backeb.) Ostolaza }\end{array}$ & Liege & 1973 & 1983 & $\begin{array}{l}\text { arrived as H. zonatus; } 2 \\
\text { synonyms }\end{array}$ \\
\hline Haageocereus australis Backeb. & Liege & 1973 & 1977 & $\begin{array}{l}\text { arrived as H. multicolor- } \\
\text { ispinus; one of } 2 \text { synonyms }\end{array}$ \\
\hline Haageocereus decumbens (Vaupel) Backeb. & Liege & 1973 & 1983 & $\begin{array}{l}\text { arrived as H. ambiguus } \\
\text { var. reductus }\end{array}$ \\
\hline Haageocereus fascicularis (Meyen) F. Ritter & $\begin{array}{l}\text { Vienna-Belve- } \\
\text { dere }\end{array}$ & 1971 & 1983 & $\begin{array}{l}\text { arrived as Weberbauerocer- } \\
\text { eus fascicularis; one of } 3 \\
\text { synonyms }\end{array}$ \\
\hline Haageocereus fulvus F. Ritter & Liege & 1973 & 1983 & $\begin{array}{l}\text { arrived as H. acranthus } \\
\text { var. fortalezenis }\end{array}$ \\
\hline
\end{tabular}


S30 Sandev, D. et al.: Plethora of plants - collections of the Botanical Garden, Faculty of Science, University of Zagreb (2)

\begin{tabular}{|c|c|c|c|c|}
\hline Scientific name & $\begin{array}{l}\text { City of plant } \\
\text { material origin }\end{array}$ & $\begin{array}{l}\text { Year of } \\
\text { ordering/ } \\
\text { purchasing }\end{array}$ & $\begin{array}{l}\text { Year when plant } \\
\text { was last recorded } \\
\text { in collection }\end{array}$ & Notes and remarks \\
\hline \multirow[t]{2}{*}{ Haageocereus multangularis (Haw.) F. Ritter } & Liege & 1973 & 1980 & $\begin{array}{l}\text { arrived as H. pseudomela- } \\
\text { nostele var. carminiflorus; } \\
\text { 20+ synonyms }\end{array}$ \\
\hline & Monaco & 1991 & 1993 & $\begin{array}{l}\text { arrived as H. pseudomela- } \\
\text { nostele }\end{array}$ \\
\hline Haageocereus pacalaensis Backeb. & Monaco & 1993 & 1993 & $\begin{array}{l}\text { arrived as H. pseudoversi- } \\
\text { color; } 20+\text { synonyms }\end{array}$ \\
\hline \multirow[t]{2}{*}{ Haageocereus seticeps Rauh \& Backeb. } & Liege & 1973 & 1975 & \\
\hline & Liege & 1973 & 1982 & $\begin{array}{l}\text { arrived as } H \text {. seticeps var. } \\
\text { robustispinus }\end{array}$ \\
\hline \multirow[t]{2}{*}{ Hariota salicornioides DC. } & $\mathrm{nn}$ & 1953 & 1971 & \\
\hline & Bergen & 1960 & 1961 & \\
\hline Harrisia adscendens (Gürke) Britton \& Rose & Barcelona & $\begin{array}{l}1960 \& 1961 \\
\& 1967 \& \\
1971\end{array}$ & $\begin{array}{l}1978 \& 1989 \& \\
1968 \& 1978\end{array}$ & $\begin{array}{l}\text { arrived as Eriocereus } \\
\text { adscendens }\end{array}$ \\
\hline Harrisia balansae (K. Schum.) N.P. Taylor \& Zappi & Erlangen & 1965 & 1963 & \\
\hline \multirow[t]{7}{*}{ Harrisia bonplandii (Parm.) Britton \& Rose } & Erlangen & $1961 \& 1965$ & 1968 \& 1983 & $\begin{array}{l}\text { arrived as Eriocereus } \\
\text { guelichii; one of } 20+ \\
\text { synonyms }\end{array}$ \\
\hline & nn & unknown & 1968 & \\
\hline & Friburg & 1957 & 1983 & arrived as H. bonplandii \\
\hline & Erlangen & 1965 & 1970 & \\
\hline & $\mathrm{nn}$ & $1949 \& 1950$ & no data \& 1982 & $\begin{array}{l}\text { arrived as Cereus } \\
\text { bonplandii; from cuttings } \\
\text { (1949) }\end{array}$ \\
\hline & Berlin & $\begin{array}{l}1974 \& 1979 \\
\& 1996\end{array}$ & $\begin{array}{l}1983 \& \text { \& } 1989 \& \\
2000\end{array}$ & \\
\hline & Berlin & $1974 \& 1979$ & $1983 \& 1983$ & $\begin{array}{l}\text { arrived as Eriocereus } \\
\text { bonplandii }\end{array}$ \\
\hline Harrisia $\times$ jusbertii (Rebut \& K. Schum.) Frič & Vienna & 1964 & 1990 & $\begin{array}{l}\text { arrived as Cereus jusbertii; } \\
\text { one of } 2 \text { synonyms; from } \\
\text { cuttings }\end{array}$ \\
\hline \multirow[t]{2}{*}{ Harrisia martinii (Labour.) Britton } & nn & $\begin{array}{l}1953 \& \text { in } \\
\text { collection in } \\
1968\end{array}$ & $1990 \& 1972$ & $\begin{array}{l}\text { arrived as Cereus martinii; } \\
\text { from cuttings (1953); one } \\
\text { of } 2 \text { synonyms }\end{array}$ \\
\hline & Leipzig & 1995 & 2000 & \\
\hline \multirow[t]{2}{*}{$\begin{array}{l}\begin{array}{l}\text { Harrisia pomanensis (F.A.C. Weber ex K. Schum.) } \\
\text { Britton \& Rose }\end{array} \\
\end{array}$} & nn & 1949 & 1961 & 2 synonyms, from cuttings \\
\hline & Barcelona & 1971 & 1990 & \\
\hline Harrisia tetracantha (Labour.) D.R. Hunt & Barcelona & 1980 & 1993 & $\begin{array}{l}\text { arrived as Roseocereus } \\
\text { tetracanthus; one of } 5 \\
\text { synonyms }\end{array}$ \\
\hline \multirow[t]{2}{*}{$\begin{array}{l}\text { Harrisia tortuosa (J. Forbes ex Otto \& A. Dietr.) } \\
\text { Britton \& Rose }\end{array}$} & Rouen & $\begin{array}{l}1955 \& 1957 \\
\& 1959\end{array}$ & $\begin{array}{l}1990 \& 1989 \& \\
1961\end{array}$ & 2 synonyms \\
\hline & Halle & 1957 & 1963 & \\
\hline \multirow[t]{2}{*}{ Hatiora gaertneri (Regel) Barthlott } & Uppsala & 1963 & 1972 & $\begin{array}{l}\text { arrived as Epiphyllopsis } \\
\text { gaertneri; one of } 2 \\
\text { synonyms }\end{array}$ \\
\hline & Nancy & 1958 & 1964 & $\begin{array}{l}\text { arrived as Rhipsalidopsis } \\
\text { gaertneri }\end{array}$ \\
\hline Hatiora salicornioides Britton \& Rose & Bucharest & 1985 & 1985 & 2 synonyms \\
\hline Heliocereus speciosus (Cav.) Britton \& Rose & Krakow & 1961 & 1971 & 3 synonyms \\
\hline \multirow[t]{5}{*}{ Hylocereus setaceus (Salm-Dyck ex DC.) Ralf Bauer } & Erlangen & $\begin{array}{l}1960 \& 1965 \\
\& 1968\end{array}$ & $\begin{array}{l}1980 \& 1989 \& \\
1978\end{array}$ & 4 synonyms \\
\hline & nn & unknown & 1968 & \\
\hline & Goettingen & $1970 \& 1971$ & $1983 \& 1983$ & \\
\hline & Jena & 1967 & 1989 & \\
\hline & Wroclaw & $1985 \& 1988$ & $1989 \& 2000$ & \\
\hline \multirow[t]{4}{*}{ Hylocereus triangularis (L.) Britton \& Rose } & Liege & 1985 & 1989 & arrived as H. cubensis \\
\hline & Antwerpen & $\begin{array}{l}1962 \& 1971 \\
\& 1973\end{array}$ & $\begin{array}{l}1983 \& 1972 \& \\
1983\end{array}$ & $\begin{array}{l}\text { arrived as Cereus triangula- } \\
\text { ris; one of } 15+\text { synonyms }\end{array}$ \\
\hline & Besancon & 1971 & 1983 & \\
\hline & Berlin & 1973 & 2000 & \\
\hline Krainzia longiflora (Britton \& Rose) Backeb. & Goettingen & 1985 & 1990 & $\begin{array}{l}\text { syn. Mammillaria longiflora } \\
\text { subsp. longiflora }\end{array}$ \\
\hline
\end{tabular}




\begin{tabular}{|c|c|c|c|c|}
\hline Scientific name & $\begin{array}{l}\text { City of plant } \\
\text { material origin }\end{array}$ & $\begin{array}{l}\text { Year of } \\
\text { ordering/ } \\
\text { purchasing }\end{array}$ & $\begin{array}{l}\text { Year when plant } \\
\text { was last recorded } \\
\text { in collection }\end{array}$ & Notes and remarks \\
\hline \multirow[t]{3}{*}{ Lepismium cruciforme (Vell.) Miq. } & Muenster & 1965 & 1983 & 3 synonyms \\
\hline & $\mathrm{nn}$ & 1954 & 1984 & $\begin{array}{l}\text { arrived as Rhipsalis } \\
\text { myosurus; from cuttings }\end{array}$ \\
\hline & Szeged & $1983 \& 1996$ & $2005 \& 1997$ & arrived as L. cruciforme \\
\hline Lepismium houlletianum (Lem.) Barthlott & Porto & 1971 & 1990 & $\begin{array}{l}\text { arrived as Rhipsalis } \\
\text { houlletiana; one of } 3 \\
\text { synonyms }\end{array}$ \\
\hline \multirow[t]{5}{*}{ Lepismium ianthothele (Monv.) Barthlott } & Berlin & $1964 \& 1968$ & 1978 \& 1980 & $\begin{array}{l}\text { arrived as Pfeiffera } \\
\text { ianthothele; one of } 10+ \\
\text { synonyms }\end{array}$ \\
\hline & Erlangen & 1963 & 1971 & \\
\hline & Wageningen & 1972 & 1983 & \\
\hline & Prague & 1989 & 2010 & \\
\hline & Cluj-Napoca & 1995 & 2010 & \\
\hline Lepismium lorentzianum (Griseb.) Barthlott & Muenster & $1964 \& 1965$ & $1993 \& 1971$ & $\begin{array}{l}\text { arrived as Rhipsalis } \\
\text { lorentziana }\end{array}$ \\
\hline Lepismium lumbricoides (Lem.) Barthlott & Goettingen & 1966 & 1984 & $\begin{array}{l}\text { arrived as Rhipsalis } \\
\text { lumbricoides; one of } 4 \\
\text { synonyms }\end{array}$ \\
\hline \multirow[t]{3}{*}{ Lepismium warmingianum (K. Schum.) Barthlott } & $\mathrm{nn}$ & 1954 & 1968 & $\begin{array}{l}\text { arrived as Rhipsalis } \\
\text { warmingiana, from } \\
\text { cuttings }\end{array}$ \\
\hline & Bergen & 1960 & 1962 & \\
\hline & Essen & & 1977 & \\
\hline \multirow[t]{3}{*}{ Leuchtenbergia principis Hook. } & Vienna & 1964 & 1964 & arrived as planta viva \\
\hline & Halle & 1966 & 1968 & \\
\hline & Dresden & 1995 & 2016 & \\
\hline \multirow{3}{*}{ Lobivia backebergii (Werderm.) Backeb. } & Meinz & 1967 & 2000 & $15+$ synonyms \\
\hline & Vienna & 1967 & 1978 & \\
\hline & Linz & 1963 & 1982 & \\
\hline \multirow[t]{2}{*}{$\begin{array}{l}\text { Lobivia backebergii var. wrightiana (Backeb.) } \\
\text { Rausch }\end{array}$} & Halle & 1963 & 1975 & $\begin{array}{l}\text { arrived as L. wrightiana; } \\
\text { one of } 3 \text { synonyms }\end{array}$ \\
\hline & Jibou & 1989 & 1990 & \\
\hline Lobivia bruchii Britton \& Rose & Berlin & 1953 & 1982 & $\begin{array}{l}\text { arrived as Echinopsis } \\
\text { bruchii; one of } 2 \text { synonyms }\end{array}$ \\
\hline \multirow[t]{2}{*}{$\begin{array}{l}\text { Lobivia densispina Werderm. ex Backeb. \& F.M. } \\
\text { Knuth f. albolanata }\end{array}$} & Linz & 1958 & 1971 & $\begin{array}{l}\text { arrived as L. pectinifera } \mathrm{f} . \\
\text { albolanata }\end{array}$ \\
\hline & Vienna & 1985 & 1990 & \\
\hline Lobivia drijveriana Backeb. (var. astranthema) & Halle & 1966 & 1971 & $\begin{array}{l}\text { two synonims; from } \\
\text { cuttings }\end{array}$ \\
\hline \multirow[t]{4}{*}{ Lobivia pygmaea (R.E. Fr.) Backeb. } & Linz & 1963 & 1982 & $\begin{array}{l}\text { arrived as Mediolobivia } \\
\text { pygmaea; one of 10+ } \\
\text { synonyms }\end{array}$ \\
\hline & Gent & $1968 \& 1970$ & $1982 \& 1982$ & \\
\hline & Goettingen & 1989 & 1990 & \\
\hline & Poznan & 1993 & 1993 & \\
\hline Lophocereus schottii (Engelm.) Britton \& Rose & Barcelona & 1980 & 1989 & 3 synonyms \\
\hline $\begin{array}{l}\text { Lophocereus schottii var. australis (K. Brandegee) } \\
\text { Borg }\end{array}$ & $\begin{array}{l}\text { San Marino } \\
\text { (California) }\end{array}$ & 1976 & 1993 & 3 synonyms \\
\hline Lophophora diffusa (Croizat) Bravo & Debrecen & 1980 & 1983 & $\begin{array}{l}\text { arrived as L. lutea; one of } 2 \\
\text { synonyms }\end{array}$ \\
\hline \multirow[t]{5}{*}{$\begin{array}{l}\text { Lophophora williamsii (Lem. ex Salm-Dyck) J.M. } \\
\text { Coult. }\end{array}$} & Debrecen & 1980 & 1983 & $\begin{array}{l}\text { arrived as L. jourdaniana; } \\
\text { one of } 5 \text { synonyms }\end{array}$ \\
\hline & nn & $\begin{array}{l}\text { in collection } \\
\text { in } 1961\end{array}$ & 1983 & \\
\hline & Lokrum & 1968 & 1968 & \\
\hline & Turku & 1967 & 1983 & \\
\hline & Jibou & 1989 & 2003 & \\
\hline Maihueniopsis darwinii (Hensl.) F.Ritter & Gent & $1962 \& 1963$ & $1978 \& 1979$ & $\begin{array}{l}\text { arrived as Tephrocactus } \\
\text { darwinii; one of } 3 \\
\text { synonyms }\end{array}$ \\
\hline Maihueniopsis ovata (Pfeiff.) F. Ritter & Beograd & 1965 & 1978 & $\begin{array}{l}\text { arrived as Tephrocactus } \\
\text { ovatus; one of } 4 \text { synonyms }\end{array}$ \\
\hline
\end{tabular}


S32 Sandev, D. et al.: Plethora of plants - collections of the Botanical Garden, Faculty of Science, University of Zagreb (2)

\begin{tabular}{|c|c|c|c|c|}
\hline Scientific name & $\begin{array}{l}\text { City of plant } \\
\text { material origin }\end{array}$ & $\begin{array}{l}\text { Year of } \\
\text { ordering/ } \\
\text { purchasing }\end{array}$ & $\begin{array}{l}\text { Year when plant } \\
\text { was last recorded } \\
\text { in collection }\end{array}$ & Notes and remarks \\
\hline Mammillaria backebergiana F.G. Buchenau & Berlin & 1979 & 1989 & $\begin{array}{l}\text { arrived as } M \text {. fertilis; one } \\
\text { of } 2 \text { synonyms }\end{array}$ \\
\hline \multirow[t]{2}{*}{ Mammillaria baumii Boed. } & Linz & 1981 & 1981 & $\begin{array}{l}\text { arrived as Dolichothele } \\
\text { baumii }\end{array}$ \\
\hline & Barcelona & 1968 & 1982 & \\
\hline \multirow[t]{4}{*}{ Mammillaria bocasana Poselger } & Berlin & $\begin{array}{l}1953 \& 1974 \\
\& 1979\end{array}$ & $\begin{array}{l}1971 \& 1983 \& \\
1982\end{array}$ & 3 synonyms \\
\hline & Muenster & 1967 & no data & \\
\hline & Sarvar & 1973 & 1982 & \\
\hline & Goettingen & 1985 & 1990 & \\
\hline \multirow[t]{8}{*}{$\begin{array}{l}\text { Mammillaria bocasana subsp. eschauzieri (J.M. } \\
\text { Coult.) Fitz Maurice \& B. Fitz Maur. }\end{array}$} & Berlin & $1960 \& 1962$ & $1971 \& 1968$ & $\begin{array}{l}\text { arrived as M. hirsuta; one } \\
\text { of 5+ synonyms }\end{array}$ \\
\hline & Graz & 1975 & 1990 & arrived as $M$. knebeliana \\
\hline & nn & 1962 & 1966 & arrived as M. kunzeana \\
\hline & $\mathrm{nn}$ & 1961 & 1971 & \\
\hline & nn & $\begin{array}{l}\text { in collection } \\
\text { in } 1968\end{array}$ & 1971 & \\
\hline & Berlin & $1955 \& 1963$ & $1978 \& 1980$ & arrived as M. longicoma \\
\hline & Nantes & 1962 & 1976 & \\
\hline & Karlsruhe & 1965 & 1975 & \\
\hline \multirow[t]{4}{*}{ Mammillaria carnea Zucc. ex Pfeiff. } & Nantes & $1960 \& 1962$ & $1961 \& 1968$ & 5+ synonyms \\
\hline & Karlsruhe & $1965 \& 1969$ & $1968 \& 1977$ & \\
\hline & Erlangen & 1965 & 1993 & arrived as M. orcuttii \\
\hline & Linz & 1967 & 1980 & \\
\hline \multirow[t]{2}{*}{ Mammillaria cirrhifera Mart. } & Palermo & 1959 & 1989 & syn. Cactus cirrhifer \\
\hline & Nantes & 1961 & 1990 & \\
\hline \multirow[t]{3}{*}{ Mammillaria columbiana Salm-Dyck } & Halle & 1966 & 1971 & 2 synonyms \\
\hline & Košice & 1978 & 1982 & \\
\hline & Greifswald & 1989 & 1990 & \\
\hline \multirow[t]{8}{*}{ Mammillaria compressa DC. } & Berlin & 1978 & 1982 & $\begin{array}{l}\text { arrived as M. esseriana; } \\
\text { one of } 5+\text { synonyms }\end{array}$ \\
\hline & Gent & 1963 & 1989 & arrived as M. tolimensis \\
\hline & Coimbra & 1955 & no data & arrived as $M$. compressa \\
\hline & Muenchen & 1989 & 1997 & \\
\hline & Gent & 1968 & 2004 & \\
\hline & nn & $\begin{array}{l}\text { in collection } \\
\text { in } 1961\end{array}$ & 1980 & \\
\hline & Muenchen & 1967 & 1989 & arrived as M. seitziana \\
\hline & Muenster & 1968 & 1989 & \\
\hline \multirow[t]{6}{*}{ Mammillaria coronaria (Willd.) Haw. } & Berlin & 1967 & 1982 & $\begin{array}{l}\text { arrived as } M . \text { neocoronaria; } \\
\text { one of } 3 \text { synonvms }\end{array}$ \\
\hline & Tuebingen & $1967 \& 1970$ & $1972 \& 1976$ & \\
\hline & Wageningen & 1972 & 1982 & \\
\hline & Nantes & 1962 & 1974 & \\
\hline & Palermo & $\begin{array}{l}1957 \& 1959 \\
\& 1961 \& \\
1962 \& 1964\end{array}$ & $\begin{array}{l}1989 \& 1966 \& \& \\
1989 \& 1977 \& \& \\
1968\end{array}$ & arrived as M. coronata \\
\hline & Košice & $1978 \& 1980$ & $1983 \& 1989$ & \\
\hline \multirow[t]{3}{*}{ Mammillaria crebrispina DC. } & Nantes & 1962 & 1993 & syn. Cactus crebrispinus \\
\hline & Palermo & $\begin{array}{l}1962 \& \& \\
1964 \& 1965\end{array}$ & $\begin{array}{l}1989 \& 1968 \& \\
1980\end{array}$ & \\
\hline & Berlin & 1965 & 1989 & \\
\hline \multirow[t]{3}{*}{ Mammillaria crinita DC. } & nn & 1962 & 1975 & $\begin{array}{l}\text { arrived as M. schelhasei; } \\
\text { one of } 3 \text { synonyms }\end{array}$ \\
\hline & Berlin & 1965 & 1978 & \\
\hline & Vienna & 1965 & 1978 & \\
\hline \multirow[t]{5}{*}{$\begin{array}{l}\text { Mammillaria crinita subsp. leucantha (Boed.) D.R. } \\
\text { Hunt }\end{array}$} & Nantes & $1961 \& 1962$ & $1971 \& 1968$ & $\begin{array}{l}\text { arrived as } M . \text { aurihamata; } \\
\text { one of } 4 \text { synonyms }\end{array}$ \\
\hline & Nantes & 1962 & 1975 & arrived as M. erectohamata \\
\hline & Gent & 1962 & 1975 & \\
\hline & $\mathrm{nn}$ & unknown & 1968 & \\
\hline & Nantes & 1965 & 1978 & arrived as M. leucantha \\
\hline
\end{tabular}




\begin{tabular}{|c|c|c|c|c|}
\hline Scientific name & $\begin{array}{l}\text { City of plant } \\
\text { material origin }\end{array}$ & $\begin{array}{l}\text { Year of } \\
\text { ordering/ } \\
\text { purchasing }\end{array}$ & \begin{tabular}{|l|} 
Year when plant \\
was last recorded \\
in collection
\end{tabular} & Notes and remarks \\
\hline & nn & $\begin{array}{l}\text { in collection } \\
\text { in } 1968\end{array}$ & 1971 & \\
\hline \multirow[t]{2}{*}{$\begin{array}{l}\text { Mammillaria crinita subsp. wildii (A. Dietr.) D.R. } \\
\text { Hunt }\end{array}$} & nn & $\begin{array}{l}\text { in collection } \\
\text { in } 1961\end{array}$ & 1980 & arrived as $M$. wildii \\
\hline & Debrecen & 1980 & 1993 & \\
\hline \multirow[t]{2}{*}{ Mammillaria crucigera Mart. } & Berlin & 1964 & 1977 & syn. Cactus cruciger \\
\hline & Jibou & 1989 & 1990 & \\
\hline \multirow[t]{5}{*}{$\begin{array}{l}\text { Mammillaria decipiens Scheidw. subsp. } \\
\text { camptotricha (Dams) D.R. Hunt }\end{array}$} & Gent & $1963 \& 1967$ & $1977 \& 1972$ & $\begin{array}{l}\text { arrived as Dolichothele } \\
\text { camptotricha; one of } 3 \\
\text { synonyms }\end{array}$ \\
\hline & Karlsruhe & 1971 & 1975 & \\
\hline & nn & $\begin{array}{l}\text { in collection } \\
\text { in } 1961\end{array}$ & 1968 & arrived as M. camptotricha \\
\hline & Gent & 1963 & 1968 & \\
\hline & Gent & 1963 & 1968 & $\begin{array}{l}\text { arrived as Pseudomammil- } \\
\text { laria camptotricha }\end{array}$ \\
\hline Mammillaria densispina (J.M. Coult.) Orcutt & Nantes & 1962 & 1976 & 4 synonyms \\
\hline \multirow[t]{2}{*}{ Mammillaria discolor Haw. } & Berlin & 1979 & 1982 & $\begin{array}{l}\text { arrived as } M \text {. amoena; one } \\
\text { of } 2 \text { synonyms }\end{array}$ \\
\hline & Monaco & 1989 & 1990 & \\
\hline Mammillaria elongata DC. & Debrecen & 1980 & 2003 & 6 synonyms \\
\hline \multirow[t]{2}{*}{$\begin{array}{l}\text { Mammillaria elongata var. stella-aurata (Mart.) K. } \\
\text { Schum. }\end{array}$} & nn & $\begin{array}{l}1947 \& \text { in } \\
\text { collection in } \\
1961\end{array}$ & $1971 \& 1961$ & $\begin{array}{l}\text { from cuttings (1961); } 2 \\
\text { synonyms }\end{array}$ \\
\hline & Besancon & $1967 \& 1969$ & $1977 \& 1971$ & \\
\hline \multirow[t]{2}{*}{ Mammillaria erythrosperma Boed. } & Berlin & 1965 & 1968 & 2 synonyms \\
\hline & Beograd & 1965 & 1968 & \\
\hline \multirow[t]{3}{*}{$\begin{array}{l}\text { Mammillaria formosa Galeotti ex Scheidw. subsp. } \\
\text { chionocephala (J.A. Purpus) D.R. Hunt }\end{array}$} & Karlsruhe & $1964 \& 1965$ & $1975 \& 1977$ & $\begin{array}{l}\text { arrived as } M . \text { chionocepha- } \\
\text { la; one of } 3 \text { synonyms }\end{array}$ \\
\hline & Linz & $1964 \& 1965$ & $1977 \& 1983$ & arrived as $M$. ritteriana \\
\hline & Halle & 1973 & 1975 & \\
\hline \multirow[t]{3}{*}{$\begin{array}{l}\text { Mammillaria formosa subsp. pseudocrucigera (R.T. } \\
\text { Craig) D.R. Hunt }\end{array}$} & $\operatorname{Linz}$ & $1965 \& 1970$ & $1976 \& 1976$ & $\begin{array}{l}\text { arrived as } M . \text { sempervivi; } \\
\text { one of } 5 \text { synonyms }\end{array}$ \\
\hline & Muenchen & 1967 & 1975 & \\
\hline & $\mathrm{nn}$ & unknown & 1975 & \\
\hline Mammillaria geminispina Haw. & nn & $\begin{array}{l}1951 \text { \& in } \\
\text { collection in } \\
1963 \& 1963\end{array}$ & $\begin{array}{l}1961 \& 2003 \& \\
1977\end{array}$ & $\begin{array}{l}\text { arrived as } M . \text { nivea; one of } \\
3 \text { synonyms }\end{array}$ \\
\hline \multirow[t]{5}{*}{ Mammillaria gracilis Pfeiff. } & Lisbon & $1955 \& 1963$ & $1995 \& 2003$ & 4 synonyms \\
\hline & nn & $\begin{array}{l}\text { in collection } \\
\text { in } 1961\end{array}$ & 2010 & \\
\hline & $\mathrm{nn}$ & 1963 & 1971 & \\
\hline & Liege & 1984 & 1989 & \\
\hline & nn & 1993 & no data & \\
\hline \multirow[t]{3}{*}{ Mammillaria grahamii Engelm. } & $\begin{array}{l}\text { San Marino } \\
\text { (California) }\end{array}$ & 1992 & 1993 & $\begin{array}{l}\text { arrived as M. microcarpa; } \\
\text { one of } 10+\text { synonyms }\end{array}$ \\
\hline & Berlin & 1962 & 1978 & arrived as M. sheldonii \\
\hline & Halle & 1966 & 1989 & \\
\hline \multirow[t]{4}{*}{ Mammillaria haageana Pfeiff. } & Palermo & 1959 & 1989 & $\begin{array}{l}\text { arrived as } M . \text { donatii; one } \\
\text { of } 5 \text { synonyms }\end{array}$ \\
\hline & Debrecen & 1980 & 1983 & arrived as M. haageana \\
\hline & Berlin & 1979 & 1983 & arrived as M. kunthii \\
\hline & Nantes & 1986 & 1993 & \\
\hline \multirow[t]{3}{*}{ Mammillaria haageana subsp. elegans D.R. Hunt } & $\begin{array}{l}\text { Hann. } \\
\text { Muenden }\end{array}$ & 1975 & 1982 & $\begin{array}{l}\text { arrived as } M . \text { elegans; one } \\
\text { of } 4 \text { synonyms }\end{array}$ \\
\hline & Linz & 1978 & 1982 & \\
\hline & Barcelona & 1991 & 1993 & \\
\hline \multirow[t]{5}{*}{ Mammillaria hahniana Werderm. } & Sarvar & 1973 & 1993 & $\begin{array}{l}\text { arrived as } M . \text { bravoae; one } \\
\text { of } 4 \text { synonyms }\end{array}$ \\
\hline & Debrecen & 1980 & 1981 & $\begin{array}{l}\text { arrived as M. hahniana var. } \\
\text { giselana }\end{array}$ \\
\hline & Lokrum & 1970 & 1979 & arrived as M. hahniana \\
\hline & Linz & 1967 & 1971 & \\
\hline & Vienna & 1967 & 1980 & \\
\hline
\end{tabular}


S34 Sandev, D. et al.: Plethora of plants - collections of the Botanical Garden, Faculty of Science, University of Zagreb (2)

\begin{tabular}{|c|c|c|c|c|}
\hline Scientific name & $\begin{array}{l}\text { City of plant } \\
\text { material origin }\end{array}$ & $\begin{array}{l}\text { Year of } \\
\text { ordering/ } \\
\text { purchasing }\end{array}$ & $\begin{array}{l}\text { Year when plant } \\
\text { was last recorded } \\
\text { in collection }\end{array}$ & Notes and remarks \\
\hline & Berlin & 1967 & 1975 & \\
\hline & Košice & 1978 & 1990 & \\
\hline & Halle & $1998 \& 1999$ & $2003 \& 2003$ & \\
\hline \multirow[t]{3}{*}{$\begin{array}{l}\text { Mammillaria hahniana subsp. mendeliana (Bravo) } \\
\text { D.R. Hunt }\end{array}$} & Karlsruhe & $1963 \& 1964$ & $1968 \& 1975$ & $\begin{array}{l}\text { arrived as } M . \text { mendeliana; } \\
\text { one of } 3 \text { synonyms }\end{array}$ \\
\hline & Muenchen & 1967 & 2003 & \\
\hline & Turku & 1967 & 1975 & \\
\hline \multirow[t]{3}{*}{$\begin{array}{l}\text { Mammillaria hahniana subsp. woodsii (R.T. Craig) } \\
\text { D.R. Hunt }\end{array}$} & Karlsruhe & 1964 & 1990 & arrived as $M$. woodsii \\
\hline & Gent & $1965 \& 1968$ & $1968 \& 1977$ & \\
\hline & Muenchen & 1966 & 1983 & \\
\hline \multirow[t]{3}{*}{ Mammillaria hamata Lehm. } & Berlin & $1965 \& 1979$ & $1973 \& 1983$ & 3 synonyms \\
\hline & $\mathrm{nn}$ & unknown & 1961 & \\
\hline & Essen & 1968 & 1971 & \\
\hline \multirow[t]{2}{*}{ Mammillaria heeriana } & Nantes & 1962 & 1975 & \\
\hline & Karlsruhe & 1965 & 1975 & \\
\hline \multirow[t]{7}{*}{ Mammillaria karwinskiana Mart. } & Palermo & 1962 & 1989 & 5 synonyms \\
\hline & Muenchen & $1967 \& 1970$ & $1989 \& 1971$ & \\
\hline & Basel & 1970 & 1975 & \\
\hline & Gent & 1968 & 1993 & arrived as $M$. neomystax \\
\hline & Berlin & 1965 & 2003 & arrived as M. ebenacantha \\
\hline & Muenster & 1968 & 1977 & arrived as $M$. confusa \\
\hline & Berlin & 1959 & 1963 & arrived as $M$. fischeri \\
\hline $\begin{array}{l}\text { Mammillaria karwinskiana subsp. collinsii (Britton } \\
\text { \& Rose) D.R. Hunt }\end{array}$ & Košice & 1978 & 1983 & $\begin{array}{l}\text { arrived as M. collinsii; one } \\
\text { of } 3 \text { synonyms }\end{array}$ \\
\hline \multirow[t]{2}{*}{$\begin{array}{l}\text { Mammillaria karwinskiana subsp. nejapensis (R.T. } \\
\text { Craig \& E.Y. Dawson) D.R. Hunt }\end{array}$} & Halle & 1979 & 1990 & $\begin{array}{l}\text { arrived as M. nejapensis; } \\
\text { one of } 2 \text { synonyms }\end{array}$ \\
\hline & Cluj-Napoca & 2005 & 2010 & \\
\hline \multirow[t]{3}{*}{ Mammillaria klissingiana Boed. } & Berlin & 1965 & 1977 & arrived as M. brauneana \\
\hline & Vienna & 1965 & 1978 & \\
\hline & Halle & 1979 & 1983 & syn. M. brauneana \\
\hline \multirow[t]{2}{*}{ Mammillaria lloydii (Britton \& Rose) Orcutt } & Basel & $\begin{array}{l}1959 \& 1970 \\
\& 1975\end{array}$ & $\begin{array}{l}1975 \& 1979 \& \\
1989\end{array}$ & syn. Neomammillaria lloydii \\
\hline & Turku & 1967 & 1976 & \\
\hline \multirow[t]{4}{*}{ Mammillaria longimamma DC. } & Halle & 1965 & 1978 & $\begin{array}{l}\text { arrived as Dolichothele } \\
\text { longimamma }\end{array}$ \\
\hline & Barcelona & 1968 & 1990 & \\
\hline & nn & 1958 & 1978 & \\
\hline & Debrecen & 1980 & 1990 & \\
\hline \multirow[t]{17}{*}{ Mammillaria magnimamma Haw. } & Muenster & 1968 & 1975 & $\begin{array}{l}\text { arrived as M. bicornata, } \\
\text { one of } 10+\text { synonyms }\end{array}$ \\
\hline & $\mathrm{nn}$ & 1951 & 1990 & arrived as M. centricirrha \\
\hline & Palermo & 1963 & 1989 & \\
\hline & Berlin & 1965 & 2000 & $\begin{array}{l}\text { arrived as M. centricirrha } \\
\text { var.bockii }\end{array}$ \\
\hline & Palermo & $\begin{array}{l}1952 \& 1957 \\
\& 1961 \& \\
1962\end{array}$ & $\begin{array}{l}1982 \& 1981 \& \\
1993 \& 1981\end{array}$ & arrived as M. gladiata \\
\hline & nn & $\begin{array}{l}\text { in collection } \\
\text { in } 1973\end{array}$ & 1979 & \\
\hline & Genova & 1960 & 1983 & $\begin{array}{l}\text { arrived as M. magnimam- } \\
\text { ma }\end{array}$ \\
\hline & Linz & 1964 & 1989 & \\
\hline & Berlin & $1965 \& 1973$ & $1975 \& 1983$ & \\
\hline & Muenchen & 1964 & 1971 & \\
\hline & nn & unknown & 1968 & \\
\hline & Basel & 1986 & 1989 & \\
\hline & Karlsruhe & 1965 & 1989 & arrived as M. pentacantha \\
\hline & $\mathrm{nn}$ & unknown & 1968 & \\
\hline & Erlangen & 1965 & 1993 & arrived as M. phymatothele \\
\hline & Muenster & 1968 & 2000 & arrived as M. vagaspina \\
\hline & Palermo & 1964 & 1980 & $\begin{array}{l}\text { arrived as } M \text {. centricirrha } \\
\text { var. krameri hort. }\end{array}$ \\
\hline
\end{tabular}




\begin{tabular}{|c|c|c|c|c|}
\hline Scientific name & $\begin{array}{l}\text { City of plant } \\
\text { material origin }\end{array}$ & $\begin{array}{l}\text { Year of } \\
\text { ordering/ } \\
\text { purchasing }\end{array}$ & $\begin{array}{l}\text { Year when plant } \\
\text { was last recorded } \\
\text { in collection }\end{array}$ & Notes and remarks \\
\hline & nn & $\begin{array}{l}\text { in collection } \\
\text { in } 1968\end{array}$ & 1989 & $\begin{array}{l}\text { arrived as } M \text {. centricirrha } \\
\text { reflexa }\end{array}$ \\
\hline & Palermo & $1962 \& 1964$ & $1989 \& 1989$ & $\begin{array}{l}\text { arrived as } M \text {. centricirrha } \\
\text { var. schmidtii }\end{array}$ \\
\hline Mammillaria magnimamma Haw. var.divergens & Berlin & 1973 & 1989 & \\
\hline \multirow[t]{6}{*}{ Mammillaria mammillaris (L.) H. Karst. } & Linz & 1964 & 1983 & $\begin{array}{l}\text { arrived as } M \text {. simplex; one } \\
\text { of } 3 \text { synonyms }\end{array}$ \\
\hline & Muenchen & 1966 & 1968 & \\
\hline & Turku & 1967 & 1975 & \\
\hline & Jibou & 1989 & 1993 & \\
\hline & Gent & $1962 \& 1963$ & $1978 \& 1989$ & arrived as $M$. caracassana \\
\hline & Muenster & 1968 & 1975 & arrived as $M$. fuliginosa \\
\hline Mammillaria matudae Bravo & Debrecen & 1980 & 1983 & syn. M. compacticaulis \\
\hline \multirow[t]{2}{*}{ Mammillaria mazatlanensis K. Schum. } & Nantes & 1970 & 1982 & 4 synonyms \\
\hline & $\begin{array}{l}\text { San Marino } \\
\text { (California) }\end{array}$ & 1984 & 1990 & arrived as $M$. occidentalis \\
\hline Mammillaria melanocentra Poselger & Linz & 1965 & 1978 & \\
\hline \multirow[t]{3}{*}{ Mammillaria microhelia Werderm. } & Linz & 1964 & 1975 & 3 synonyms \\
\hline & Gent & 1967 & 1979 & \\
\hline & Berlin & 1971 & 1977 & \\
\hline Mammillaria moelleriana Boed. & Linz & $1964 \& 1965$ & $1976 \& 1968$ & 2 synonyms \\
\hline \multirow[t]{3}{*}{ Mammillaria muehlenpfordtii C.F. Först. } & Berlin & $1965 \& 1968$ & $1978 \& 1976$ & 3 synonyms \\
\hline & Sarvar & 1973 & 1978 & arrived as M. celsiana \\
\hline & $\mathrm{nn}$ & 1975 & 1980 & \\
\hline $\begin{array}{l}\text { Mammillaria muehlenpfordtii C.F. Först. var. } \\
\text { nealeana }\end{array}$ & Turku & 1967 & 1975 & \\
\hline Mammillaria nigra Ehrenb. & Berlin & 1971 & 1983 & syn. Cactus niger \\
\hline \multirow{2}{*}{ Mammillaria nunezii (Britton \& Rose) Orcutt } & Linz & 1965 & 1971 & 6 synonyms \\
\hline & Nantes & 1965 & 1977 & \\
\hline \multirow[t]{2}{*}{$\begin{array}{l}\text { Mammillaria nunezii subsp. bella (Backeb.) D.R. } \\
\text { Hunt }\end{array}$} & Sarvar & 1973 & 1977 & arrived as $M$. bella \\
\hline & Goettingen & 1985 & 1989 & \\
\hline Mammillaria obscura Scheidw. ex Foerster & Vienna & $1960 \& 1965$ & $1975 \& 1975$ & $\begin{array}{l}\text { arrived as Neomammillaria } \\
\text { obscura }\end{array}$ \\
\hline \multirow[t]{5}{*}{ Mammillaria parkinsonii Ehrenb. } & Liege & 1973 & 1978 & $\begin{array}{l}\text { arrived as M. parkinsonii } \\
\text { var.brevispina; one of } 5+ \\
\text { synonyms }\end{array}$ \\
\hline & Porrentruy & 1985 & 1990 & \\
\hline & Muenchen & 1961 & 1983 & arrived as M. parkinsonii \\
\hline & Vienna & 1972 & 1982 & \\
\hline & Jibou & 1989 & 2003 & \\
\hline \multirow[t]{5}{*}{ Mammillaria perbella Hildm. ex K. Schum. } & nn & unknown & 1974 & arrived as $M$. cadereytensis \\
\hline & Berlin & 1973 & 1982 & \\
\hline & Berlin & 1958 & 1961 & $\begin{array}{l}\text { arrived as Neomammillaria } \\
\text { perbella }\end{array}$ \\
\hline & Košice & 1989 & 1990 & \\
\hline & Dresden & 1998 & 2000 & \\
\hline Mammillaria petterssonii Hildm. & Berlin & 1979 & 1989 & arrived as M. pilensis \\
\hline \multirow[t]{4}{*}{ Mammillaria pilispina J.A. Purpus } & Berlin & $1965 \& 1966$ & $1975 \& 1976$ & arrived as $M$. sanluisensis \\
\hline & Linz & 1966 & 1975 & \\
\hline & Turku & $1967 \& 1968$ & 1975 \& 1977 & \\
\hline & Graz & 1989 & 1993 & \\
\hline Mammillaria plumosa F.A.C. Weber & Beograd & 1965 & 1982 & $\begin{array}{l}\text { syn. Neomammillaria } \\
\text { plumosa }\end{array}$ \\
\hline \multirow[t]{6}{*}{$\begin{array}{l}\text { Mammillaria polythele Mart. subsp. durispina } \\
\text { (Boed.) D.R. Hunt }\end{array}$} & Turku & 1967 & 1975 & arrived as $M$. durispina \\
\hline & Karlsruhe & 1967 & 1978 & \\
\hline & Jibou & 1989 & 1993 & \\
\hline & Linz & $\begin{array}{l}1964 \& 1965 \\
\& 1970\end{array}$ & $\begin{array}{l}1975 \& 1975 \& \text { \& } \\
1975\end{array}$ & arrived as $M$. kelleriana \\
\hline & Meinz & 1967 & 1977 & \\
\hline & Palermo & 1966 & 1975 & arrived as $M$. subdurispina \\
\hline
\end{tabular}


S36 Sandev, D. et al.: Plethora of plants - collections of the Botanical Garden, Faculty of Science, University of Zagreb (2)

\begin{tabular}{|c|c|c|c|c|}
\hline Scientific name & $\begin{array}{l}\text { City of plant } \\
\text { material origin }\end{array}$ & $\begin{array}{l}\text { Year of } \\
\text { ordering/ } \\
\text { purchasing }\end{array}$ & $\begin{array}{l}\text { Year when plant } \\
\text { was last recorded } \\
\text { in collection }\end{array}$ & Notes and remarks \\
\hline \multirow[t]{9}{*}{$\begin{array}{l}\text { Mammillaria polythele subsp. obconella (Scheidw.) } \\
\text { D.R. Hunt }\end{array}$} & Linz & 1965 & 1976 & $\begin{array}{l}\text { arrived as } M \text {. ingens; one } \\
\text { of } 4 \text { synonyms }\end{array}$ \\
\hline & Nantes & 1973 & 1980 & \\
\hline & Berlin & 1978 & 1982 & \\
\hline & Berlin & $\begin{array}{l}1968 \& 1973 \\
\& 1979\end{array}$ & $\begin{array}{l}1971 \& 1980 \& \\
1982\end{array}$ & $\begin{array}{l}\text { arrived as M. obconella var } \\
\text { galeottii }\end{array}$ \\
\hline & Karlsruhe & 1969 & 1975 & \\
\hline & Nancy & $1971 \& 1989$ & $1975 \& 1993$ & \\
\hline & Muenster & 1968 & 1975 & arrived as M. obconella \\
\hline & Mainz & 1967 & 1975 & \\
\hline & Basel & $1970 \& 1971$ & $1975 \& 2000$ & \\
\hline \multirow[t]{29}{*}{ Mammillaria polythele subsp. polythele } & Gent & 1962 & 1966 & $\begin{array}{l}\text { arrived as } M \text {. tetracantha; } \\
\text { one of } 5+\text { synonyms }\end{array}$ \\
\hline & Vienna & $1958 \& 1960$ & $1968 \& 1968$ & \\
\hline & Karlsruhe & $1964 \& 1965$ & $1968 \& 1983$ & \\
\hline & Halle & 1966 & 1977 & \\
\hline & Palermo & 1964 & 1975 & \\
\hline & Rouen & 1965 & 1975 & \\
\hline & $\mathrm{nn}$ & unknown & 1968 & \\
\hline & Palermo & $\begin{array}{l}1957 \& 1962 \\
\& 1967\end{array}$ & $\begin{array}{l}1989 \& 1968 \& \\
1968\end{array}$ & arrived as $M$. crocidata \\
\hline & Berlin & $\begin{array}{l}1964 \& 1965 \\
\& 1967\end{array}$ & $\begin{array}{l}1983 \& 1968 \& \\
1983\end{array}$ & \\
\hline & Berlin & $1958 \& 1959$ & $1966 \& 1971$ & arrived as $M$. galeottii \\
\hline & Berlin & $1959 \& 1974$ & $1968 \& 1982$ & arrived as $M$. hidalgensis \\
\hline & Palermo & 1964 & 1975 & \\
\hline & Gent & $1963 \& 1976$ & $1966 \& 1982$ & \\
\hline & Muenchen & 1964 & 1966 & \\
\hline & Besancon & 1964 & 1971 & \\
\hline & Erlangen & 1965 & 1968 & \\
\hline & Karlsruhe & 1965 & 1982 & \\
\hline & Meinz & 1967 & 1978 & \\
\hline & Cluj-Napoca & 1973 & 1979 & \\
\hline & Joensuu & 1991 & 2003 & \\
\hline & Rouen & $1960 \& 1964$ & $1975 \& 1978$ & arrived as $M$. kewensis \\
\hline & Berlin & 1959 & 1968 & \\
\hline & Gent & 1962 & 1982 & \\
\hline & Nancy & 1989 & 1990 & \\
\hline & Jibou & 1989 & 1993 & \\
\hline & Berlin & $1960 \& 1966$ & $1976 \& 1976$ & $\begin{array}{l}\text { arrived as M. kewensis var. } \\
\text { craigiana (unknown) }\end{array}$ \\
\hline & Nantes & 1965 & 1978 & arrived as M. polythele \\
\hline & Tuebingen & 1970 & 1978 & \\
\hline & Basel & 1971 & 2003 & arrived as M. obconella \\
\hline Mammillaria poselgeri Hildm. & Berlin & 1959 & 1972 & $\begin{array}{l}\text { arrived as Cochemiea } \\
\text { poselgeri }\end{array}$ \\
\hline \multirow[t]{4}{*}{ Mammillaria prolifera (Mill.) Haw. } & $\begin{array}{l}\text { Litva (no other } \\
\text { data) }\end{array}$ & 1959 & 1983 & $\begin{array}{l}\text { arrived as M. pusilla; one } \\
\text { of } 2 \text { synonyms }\end{array}$ \\
\hline & Muenchen & 1964 & 1978 & arrived as M. prolifera \\
\hline & Litva & 1959 & 1989 & \\
\hline & nn & $\begin{array}{l}\text { in collection } \\
\text { in } 1980\end{array}$ & 2010 & \\
\hline \multirow[t]{3}{*}{$\begin{array}{l}\text { Mammillaria prolifera subsp. multiceps (Salm- } \\
\text { Dyck) U. Guzmán }\end{array}$} & Budapest & 1978 & 1989 & $\begin{array}{l}\text { arrived as M. multiceps } \\
\text { var. perpusilla; one of } 5+ \\
\text { synonyms }\end{array}$ \\
\hline & Berlin & 1973 & 2010 & $\begin{array}{l}\text { arrived as M. prolifera var. } \\
\text { texana }\end{array}$ \\
\hline & nn & $\begin{array}{l}1951 \& \text { in } \\
\text { collection in } \\
1963\end{array}$ & $1961 \& 2000$ & arrived as M. multiceps \\
\hline $\begin{array}{l}\text { Mammillaria prolifera var. multiceps (Salm-Dyck) } \\
\text { U. Guzmán }\end{array}$ & $\begin{array}{l}\text { Litva (no other } \\
\text { data) }\end{array}$ & 1959 & 1963 & $\begin{array}{l}\text { arrived as M. pusilla var. } \\
\text { multiceps; one of } 3 \\
\text { synonyms }\end{array}$ \\
\hline Mammillaria pulchella Otto ex Linke & Linz & $1955 \& 1969$ & $2003 \& 2003$ & \\
\hline
\end{tabular}




\begin{tabular}{|c|c|c|c|c|}
\hline Scientific name & $\begin{array}{l}\text { City of plant } \\
\text { material origin }\end{array}$ & $\begin{array}{l}\text { Year of } \\
\text { ordering/ } \\
\text { purchasing }\end{array}$ & $\begin{array}{l}\text { Year when plant } \\
\text { was last recorded } \\
\text { in collection }\end{array}$ & Notes and remarks \\
\hline & nn & $\begin{array}{l}1961 \& \text { in } \\
\text { collection in } \\
1975\end{array}$ & $1961 \& 1980$ & from cuttings (1961) \\
\hline \multirow[t]{7}{*}{ Mammillaria rhodantha Link \& Otto } & Berlin & $\begin{array}{l}1960 \& 1962 \\
\& 1974\end{array}$ & $\begin{array}{l}1975 \& 1971 \& \\
1982\end{array}$ & $\begin{array}{l}\text { arrived as } M \text {. fuscata; one } \\
\text { of } 5+\text { synonyms }\end{array}$ \\
\hline & Basel & 1973 & 1975 & \\
\hline & Greifswald & 1989 & 1990 & \\
\hline & Basel & $\begin{array}{l}1959 \& 1962 \\
\& 1963\end{array}$ & $\begin{array}{l}1975 \& 1975 \& \\
1975\end{array}$ & \\
\hline & Berlin & $1959 \& 1960$ & $1978 \& 1966$ & \\
\hline & Palermo & 1959 & 1963 & \\
\hline & Linz & 1965 & 1968 & \\
\hline \multirow[t]{5}{*}{$\begin{array}{l}\text { Mammillaria rhodantha subsp. aureiceps (Lem.) } \\
\text { D.R. Hunt }\end{array}$} & Linz & $1964 \& 1965$ & $1977 \& 1977$ & $\begin{array}{l}\text { arrived as M. aureiceps; } \\
\text { one of } 5+\text { +synonyms }\end{array}$ \\
\hline & Berlin & 1968 & 1977 & \\
\hline & Muenster & 1967 & 1975 & \\
\hline & Turku & 1968 & 1971 & \\
\hline & Graz & 1975 & 1982 & \\
\hline \multirow[t]{5}{*}{$\begin{array}{l}\text { Mammillaria rhodantha subsp. mollendorffiana } \\
\text { (Shurly) D.R. Hunt }\end{array}$} & Berlin & $\begin{array}{l}1958 \& 1960 \\
\& 1962 \& \\
1964 \& 1965 \\
\& 1966 \& \\
1974\end{array}$ & $\begin{array}{l}1966 \& 1971 \& \\
1976 \& 1975 \& \\
1968 \& 1968 \& \\
1982\end{array}$ & $\begin{array}{l}\text { arrived as syn. } M \text {. } \\
\text { mollendorfiana }\end{array}$ \\
\hline & Palermo & 1962 & 1964 & \\
\hline & Karlsruhe & 1964 & 1978 & \\
\hline & Muenster & $1967 \& 1968$ & $1975 \& 1972$ & \\
\hline & Jibou-Salaj & 1989 & 1993 & \\
\hline Mammillaria rhodantha var. crassispina K.Schum. & Berlin & 1976 & 1982 & syn. M. crassispina \\
\hline Mammillaria rhodantha var. rubra K.Schum. & Basel & 1971 & 1982 & \\
\hline $\begin{array}{l}\text { Mammillaria rhodantha var. sulphurea (Sancke) } \\
\text { backbg. }\end{array}$ & Basel & $1971 \& 1973$ & $1975 \& 1976$ & $\begin{array}{l}\text { arrived as M. fuscata var. } \\
\text { sulphurea; one of } 2 \\
\text { synonyms }\end{array}$ \\
\hline Mammillaria roseoalba Boed. & Berlin & 1965 & 1989 & \\
\hline \multirow[t]{2}{*}{ Mammillaria saetigera Boed. \& Tiegel } & Berlin & $1960 \& 1966$ & 1978 \& 1977 & $\begin{array}{l}\text { syn. Neomammillaria } \\
\text { saetigera }\end{array}$ \\
\hline & Halle & $1957 \& 1964$ & $1989 \& 1971$ & \\
\hline \multirow[t]{3}{*}{ Mammillaria sartorii J.A. Purpus } & Erlangen & 1965 & 1989 & 2 synonyms \\
\hline & nn & $\begin{array}{l}\text { in collection } \\
\text { in } 1968\end{array}$ & 1971 & \\
\hline & Esperance & 1970 & 1989 & \\
\hline \multirow[t]{2}{*}{ Mammillaria schiedeana Ehrenb. ex Schltdl. } & Berlin & 1959 & 1968 & 2 synonyms \\
\hline & Linz & 1964 & 1990 & \\
\hline $\begin{array}{l}\text { Mammillaria schiedeana subsp. dumetorum (J.A. } \\
\text { Purpus) D.R. Hunt }\end{array}$ & Linz & 1965 & 1990 & arrived as $M$. dumetorum \\
\hline \multirow[t]{4}{*}{ Mammillaria sinistrohamata Boed. } & Nantes & 1960 & 1968 & \\
\hline & Palermo & 1966 & 1971 & \\
\hline & Karlsruhe & 1969 & 1975 & \\
\hline & Debrecen & 1980 & 1983 & \\
\hline Mammillaria sonorensis R.T. Craig & Berlin & 1973 & 1979 & $\begin{array}{l}\text { arrived as } M \text {. tesopacensis; } \\
\text { one of } 2 \text { synonyms }\end{array}$ \\
\hline Mammillaria sphacelata Mart. & Košice & 1978 & 1993 & 3 synonyms \\
\hline \multirow[t]{4}{*}{ Mammillaria spinosissima Lem. } & Muenchen & 1967 & 1971 & 4 synonyms \\
\hline & Turku & 1967 & 1979 & \\
\hline & Liege & 1973 & 1976 & \\
\hline & Karlsruhe & 1971 & 1978 & \\
\hline $\begin{array}{l}\text { Mammillaria spinosissima var. sanguinea (F.Haage ) } \\
\text { F.Haage ex Brandegee }\end{array}$ & Nantes & 1965 & 1975 & syn. M. sanguinea \\
\hline Mammillaria stella-de-tacubaya Heese & Karlsruhe & 1965 & 1982 & $\begin{array}{l}\text { arrived as M. viereckii; } 2 \\
\text { synonyms }\end{array}$ \\
\hline \multirow[t]{4}{*}{ Mammillaria supertexta Mart. ex Pfeiff. } & Karlsruhe & 1963 & 1979 & $\begin{array}{l}\text { arrived as M. lanata; one } \\
\text { of } 2 \text { synonyms }\end{array}$ \\
\hline & Essen & 1968 & 1976 & \\
\hline & Muenchen & 1977 & 1990 & \\
\hline & Halle & 1979 & 1989 & arrived as M. supertexta \\
\hline
\end{tabular}


S38 Sandev, D. et al.: Plethora of plants - collections of the Botanical Garden, Faculty of Science, University of Zagreb (2)

\begin{tabular}{|c|c|c|c|c|}
\hline Scientific name & $\begin{array}{l}\text { City of plant } \\
\text { material origin }\end{array}$ & $\begin{array}{l}\text { Year of } \\
\text { ordering/ } \\
\text { purchasing }\end{array}$ & $\begin{array}{l}\text { Year when plant } \\
\text { was last recorded } \\
\text { in collection }\end{array}$ & Notes and remarks \\
\hline Mammillaria surculosa Boed. & Gent & 1968 & 1978 & $\begin{array}{l}\text { arrived as Dolichothele } \\
\text { surculosa }\end{array}$ \\
\hline Mammillaria tiegeliana Backeb. & Berlin & 1979 & 1982 & \\
\hline \multirow[t]{3}{*}{ Mammillaria umbrina Ehrenb. } & Berlin & $1962 \& 1976$ & $1975 \& 1982$ & 2 synonyms \\
\hline & Linz & $\begin{array}{l}1961 \& 1964 \\
\& 1966 \& \\
1972\end{array}$ & $\begin{array}{l}1961 \& 1971 \& \\
1968 \& 1982\end{array}$ & \\
\hline & Gent & 1968 & 1976 & \\
\hline \multirow[t]{2}{*}{ Mammillaria webbiana Lem. } & Berlin & 1966 & 1990 & syn. Cactus webbianus \\
\hline & Nantes & 1970 & 1989 & \\
\hline \multirow{6}{*}{ Mammillaria wiesingeri Boed. } & Linz & $1962 \& 1964$ & $196 \& \& 1975$ & 2 synonyms \\
\hline & Palermo & 1963 & 1975 & \\
\hline & Halle & 1966 & 1966 & \\
\hline & Nantes & 1965 & 1989 & \\
\hline & $\mathrm{nn}$ & $\begin{array}{l}\text { in collection } \\
\text { in } 1971\end{array}$ & 1975 & \\
\hline & Liege & 1973 & 1978 & \\
\hline Mammilloydia candida (Scheidw.) Buxb. & Pisa & 1966 & 1971 & $\begin{array}{l}\text { arrived as Mammillaria } \\
\text { candida; one of } 2 \\
\text { synonyms }\end{array}$ \\
\hline Matucana madisoniorum (Hutchison) G.D. Rowley & Dresden & 2000 & 2003 & 4 synonyms \\
\hline \multirow{3}{*}{ Mediolobivia haagei Backeb. ex Krainz } & Halle & $1957 \& 1961$ & $1980 \& 1983$ & \\
\hline & Gent & 1962 & 1968 & \\
\hline & nn & $\begin{array}{l}\text { in collection } \\
\text { in } 1971\end{array}$ & 1980 & \\
\hline \multirow[t]{6}{*}{ Melocactus bahiensis (Britton \& Rose) Luetzelb. } & Sarvar & 1973 & 1982 & 5 synonyms \\
\hline & Havana & 1986 & 1990 & \\
\hline & Reading & 1985 & 1990 & \\
\hline & Dresden & 1998 & 2003 & \\
\hline & Jibou & 1998 & 2003 & \\
\hline & Linz & 2002 & 2010 & \\
\hline \multirow[t]{4}{*}{ Melocactus neryi K. Schum. } & Halle & 1966 & 1968 & from cuttings; 3 synonyms \\
\hline & Linz & $\begin{array}{l}1962 \& 1966 \\
\& 1971 \& \\
1987\end{array}$ & $\begin{array}{l}1977 \& 1977 \& \\
1978 \& 1990\end{array}$ & \\
\hline & Vienna & $1964 \& 1965$ & $1968 \& 1977$ & \\
\hline & Dresden & $1997 \& 1998$ & $2000 \& 2000$ & \\
\hline Mesembryanthemum nodiflorum L. & Tel Aviv & 1981 & & 2 synonyms \\
\hline $\begin{array}{l}\text { Myrtillocactus geometrizans (Mart. ex Pfeiff.) } \\
\text { Console }\end{array}$ & Barcelona & $1980 \& 1995$ & $2003 \& 2003$ & 3 synonyms \\
\hline \multirow[t]{3}{*}{ Neobuxbaumia euphorbioides (Haw.) Buxb. } & Muenchen & 1967 & 2000 & $\begin{array}{l}\text { arrived as Rooksbya euphor- } \\
\text { bioides; one of } 5+ \\
\text { synonyms }\end{array}$ \\
\hline & Barcelona & 1968 & 2000 & \\
\hline & Erlangen & 1996 & 2000 & \\
\hline \multirow[t]{2}{*}{ Neobuxbaumia polylopha (DC.) Backeb. } & Barcelona & $1959 \& 1963$ & $1989 \& 2003$ & 5 synonyms \\
\hline & Lyon & 2001 & 2003 & \\
\hline \multirow[t]{2}{*}{$\begin{array}{l}\text { Neomammillaria graessneriana (Boed.) Britton \& } \\
\text { Rose }\end{array}$} & Berlin & $1971 \& 1978$ & $1980 \& 1982$ & $\begin{array}{l}\text { arrived as Mammillaria } \\
\text { graessneriana }\end{array}$ \\
\hline & Graz & 1975 & 1982 & \\
\hline \multirow[t]{6}{*}{ Neomammillaria hahniana (Werderm.) Y.Itô } & Vienna & $\begin{array}{l}1958 \& 1963 \\
\& 1965 \& \\
1967\end{array}$ & $\begin{array}{l}1968 \& 1971 \& \\
1975 \& 1978\end{array}$ & \\
\hline & Linz & 1958 & 1980 & \\
\hline & Lokrum & 1970 & 1978 & \\
\hline & Berlin & 1967 & 1978 & \\
\hline & Košice & 1978 & 1983 & \\
\hline & Barcelona & 1980 & 1983 & \\
\hline \multirow[t]{2}{*}{ Neomammillaria polythele (Mart.) Britton \& Rose } & Vienna & 1958 & 1963 & \\
\hline & Tuebingen & 1963 & 1982 & \\
\hline \multirow[t]{3}{*}{ Neomammillaria seideliana (Quehl) Britton \& Rose } & Mainz & $1966 \& 1967$ & $1977 \& 1972$ & $\begin{array}{l}\text { arrived as Mammillaria } \\
\text { seideliana }\end{array}$ \\
\hline & Berlin & 1978 & 1995 & \\
\hline & Gent & 1993 & 1993 & \\
\hline
\end{tabular}




\begin{tabular}{|c|c|c|c|c|}
\hline Scientific name & $\begin{array}{l}\text { City of plant } \\
\text { material origin }\end{array}$ & $\begin{array}{l}\text { Year of } \\
\text { ordering/ } \\
\text { purchasing }\end{array}$ & $\begin{array}{l}\text { Year when plant } \\
\text { was last recorded } \\
\text { in collection }\end{array}$ & Notes and remarks \\
\hline Neoporteria rapifera F. Ritter & Linz & 1963 & 1982 & \\
\hline \multirow[t]{2}{*}{ Neoraimondia herzogiana (Backeb.) Buxb. \& Krainz } & $\begin{array}{l}\text { Vienna-Belve- } \\
\text { dere }\end{array}$ & 1971 & 1989 & $\begin{array}{l}\text { arrived as Neocardenasia } \\
\text { herzogiana }\end{array}$ \\
\hline & $\begin{array}{l}\text { San Marino } \\
\text { (California) }\end{array}$ & 1992 & 1993 & \\
\hline Notocactus globularis F.Ritter & Goettingen & 1985 & $1985-1990$ & \\
\hline $\begin{array}{l}\text { Notocactus ottonis A. Berger var. villa-velhensis } \\
\text { Backeb. \& Voll }\end{array}$ & Košice & 1978 & 1989 & 2 synonyms \\
\hline Opuntia articulata (Pfeiff.) D.R. Hunt & Lokrum & 1970 & 1989 & $\begin{array}{l}\text { arrived as Tephrocactus } \\
\text { articulatus (v. intermis) }\end{array}$ \\
\hline Opuntia aurantiaca Lindl. & Montavideo & 1968 & 1977 & \\
\hline Opuntia beckeriana K.Schum. & Antwerpen & 1973 & 1979 & \\
\hline Opuntia boldinghii Britton \& Rose & Antwerpen & $1966 \& 1969$ & $1978 \& 1982$ & \\
\hline \multirow[t]{2}{*}{ Opuntia cochenillifera (L.) Mill. } & Nantes & 1953 & no data & $\begin{array}{l}\text { arrived as Nopalea } \\
\text { cochenillifera; one of } 3 \\
\text { synonyms; from cuttings }\end{array}$ \\
\hline & Nancy & 1989 & 1993 & \\
\hline Opuntia crassa Haw. & Barcelona & 1971 & 1982 & \\
\hline Opuntia cubensis Britton \& Rose & Antwerpen & 1971 & 1982 & $\begin{array}{l}\text { arrived as } O \text {. antillana; one } \\
\text { of } 2 \text { synonyms }\end{array}$ \\
\hline \multirow[t]{2}{*}{ Opuntia durangensis Britton \& Rose } & Barcelona & 1964 & 1990 & \\
\hline & nn & $\begin{array}{l}\text { in collection } \\
\text { in } 1968\end{array}$ & 1989 & \\
\hline Opuntia elata Link \& Otto ex Salm-Dyck & Antwerpen & 1973 & 1980 & 5 synonyms \\
\hline Opuntia engelmannii Salm-Dyck ex Engelm. & Genova & 1965 & 1971 & $\begin{array}{l}\text { arrived as } O \text {. cantabrigien- } \\
\text { sis; one of } 4 \text { synonyms }\end{array}$ \\
\hline $\begin{array}{l}\text { Opuntia engelmannii subsp. aciculata (Griffiths) U. } \\
\text { Guzmán \& Mandujano }\end{array}$ & Barcelona & 1968 & 1980 & $\begin{array}{l}\text { arriveed as O. aciculata; } \\
\text { one of } 2 \text { synonyms }\end{array}$ \\
\hline $\begin{array}{l}\text { Opuntia engelmannii subsp. lindheimeri (Engelm.) } \\
\text { U. Guzmán \& Mandujano }\end{array}$ & $\mathrm{nn}$ & 1951 & 1961 & $\begin{array}{l}\text { arrived as } O \text {. lindheimeri; } \\
\text { one of } 4 \text { synonyms }\end{array}$ \\
\hline Opuntia ficus-indica (L.) Mill. & Berlin & 1979 & 1982 & $\begin{array}{l}\text { arrived as O. paraguayen- } \\
\text { sis; one of } 4 \text { synonyms }\end{array}$ \\
\hline Opuntia fragilis (Nutt.) Haw. & Lokrum & 1967 & 1978 & from cuttings; 4 synonyms \\
\hline \multirow[t]{3}{*}{ Opuntia glaucophylla H.L.Wendl. ex Pfeiff. } & Palermo & $1953 \& 1962$ & no data \& 1982 & \\
\hline & Antibes & $\begin{array}{l}\text { in collection } \\
\text { in } 1961\end{array}$ & 1963 & \\
\hline & Halle & 1962 & 1968 & \\
\hline $\begin{array}{l}\text { Opuntia gosseliniana F.A.C. Weber var. santarita } \\
\text { (Griffiths \& Hare) L.D. Benson }\end{array}$ & Haren & 1970 & 1980 & 3 synonyms \\
\hline \multirow[t]{2}{*}{ Opuntia haematocarpa A.Berger } & nn & 1951 & 1961 & \\
\hline & Lyon & 1986 & 2003 & \\
\hline \multirow[t]{2}{*}{ Opuntia howeyi J.A. Purpus } & Tuebingen & 1952 & 1961 & from cuttings \\
\hline & Nancy & 1989 & 2003 & \\
\hline \multirow[t]{8}{*}{ Opuntia humifusa (Raf.) Raf. } & nn & $\begin{array}{l}\text { in collection } \\
\text { in } 1961\end{array}$ & 2003 & 4 synonyms \\
\hline & Antwerpen & 1971 & 1981 & \\
\hline & Basel & 1986 & 1993 & \\
\hline & Liege & 1986 & 1993 & \\
\hline & East Lansing & 1990 & 1990 & \\
\hline & Genova & 1988 & 1993 & \\
\hline & Michigan & 1999 & 2003 & \\
\hline & Genova & 1988 & 2003 & arrived as O. rafinosavii \\
\hline Opuntia inaequilaterlis A. Berger & Antwerpen & $\begin{array}{l}1961 \& 1966 \\
\& 1969 \& \\
1973\end{array}$ & $\begin{array}{l}1971 \& \text { \& } 1979 \& \\
1980 \& 1979\end{array}$ & 2 synonyms \\
\hline \multirow[t]{3}{*}{ Opuntia leucotricha DC. } & Barcelona & $\begin{array}{l}1960 \& 1961 \\
\& 1963 \& \\
1967\end{array}$ & $\begin{array}{l}1962 \& 1968 \& \\
1968 \& 1978\end{array}$ & \\
\hline & Esperance & 1970 & 1978 & \\
\hline & Antwerpen & 1970 & 1978 & \\
\hline Opuntia longispina Haw. & Gent & 1962 & 1963 & \\
\hline $\begin{array}{l}\text { Opuntia longispina var. corrugata (Salm-Dyck) } \\
\text { Backeb. }\end{array}$ & Gent & 1961 \& 1962 & 1982 \& 1982 & \\
\hline
\end{tabular}


S40 Sandev, D. et al.: Plethora of plants - collections of the Botanical Garden, Faculty of Science, University of Zagreb (2)

\begin{tabular}{|c|c|c|c|c|}
\hline Scientific name & $\begin{array}{l}\text { City of plant } \\
\text { material origin }\end{array}$ & $\begin{array}{l}\text { Year of } \\
\text { ordering/ } \\
\text { purchasing }\end{array}$ & $\begin{array}{l}\text { Year when plant } \\
\text { was last recorded } \\
\text { in collection }\end{array}$ & Notes and remarks \\
\hline \multirow[t]{8}{*}{ Opuntia microdasys (Lehm.) Pfeiff. } & Haren & 1971 & 1971 & $\begin{array}{l}\text { arrived as O. macrocalyx; } \\
\text { one of } 7 \text { synonyms }\end{array}$ \\
\hline & Gent & 1962 & 1962 & $\begin{array}{l}\text { arrived as O. microdasys } \\
\text { var. laevior }\end{array}$ \\
\hline & Barcelona & 1971 & 2003 & \\
\hline & nn & 1955 & 1961 & $\begin{array}{l}\text { arrived as O. microdasys; } \\
\text { from cuttings }\end{array}$ \\
\hline & Barcelona & $\begin{array}{l}1960 \& 1961 \\
\& 1995\end{array}$ & $\begin{array}{l}1962 \& 1980 \& \\
2003\end{array}$ & \\
\hline & $\mathrm{nn}$ & $\begin{array}{l}\text { in collection } \\
\text { in } 1968\end{array}$ & 1980 & \\
\hline & Liege & 1984 & 2003 & \\
\hline & Bucharest & 1994 & 1995 & $\begin{array}{l}\text { arrived as O. micodasys } \\
\text { var. albispina }\end{array}$ \\
\hline \multirow[t]{6}{*}{$\begin{array}{l}\text { Opuntia microdasys subsp. rufida (Engelm.) U. } \\
\text { Guzmán \& Mandujano }\end{array}$} & nn & unknown & 1961 & $5+$ synonyms \\
\hline & Barcelona & 1956 & 1963 & \\
\hline & nn & $\begin{array}{l}\text { in collection } \\
\text { in } 1968\end{array}$ & 1982 & \\
\hline & $\mathrm{nn}$ & $\begin{array}{l}\text { in collection } \\
\text { in } 1968\end{array}$ & 1971 & $\begin{array}{l}\text { arrived as O. microdasys } \\
\text { var. rufida } \mathrm{f} \text {. minima hort. }\end{array}$ \\
\hline & Barcelona & $1959 \& 1967$ & $1961 \& 1980$ & arrived as O. rufida \\
\hline & nn & $\begin{array}{l}\text { in collection } \\
\text { in } 1965\end{array}$ & 1980 & \\
\hline \multirow[t]{3}{*}{ Opuntia orbiculata Salm-Dyck ex Pfeiff. } & Besancon & 1959 & 1978 & \\
\hline & Barcelona & 1964 & 1982 & \\
\hline & Nancy & 1989 & 2003 & \\
\hline \multirow[t]{3}{*}{ Opuntia phaeacantha Engelm. } & Barcelona & 1980 & 1981 & $\begin{array}{l}\text { arrived as O. laevis } \mathrm{v} . \\
\text { canada; one of } 20+ \\
\text { synonyms }\end{array}$ \\
\hline & Bonn & $\begin{array}{l}1965 \& 1992 \\
\& 1994\end{array}$ & $\begin{array}{l}1977 \& 1997 \& \\
2003\end{array}$ & arrived as O. phaeacantha \\
\hline & Barcelona & 1964 & 1975 & \\
\hline Opuntia phaeacantha "var. albispina hort." & Barcelona & 1964 & 1968 & $15+$ synonyms \\
\hline \multirow[t]{3}{*}{$\begin{array}{l}\text { Opuntia phaeacantha var. camanchica (Engelm. \& } \\
\text { J.M. Bigelow) L.D. Benson }\end{array}$} & Halle & 1964 & 1968 & syn. O. camanchica \\
\hline & Barcelona & 1964 & 1976 & \\
\hline & Fronnleiten & 1964 & 1990 & \\
\hline Opuntia piccolomini auct. & Antwerpen & 1966 & 1968 & $\begin{array}{l}\text { unresolved name (The } \\
\text { Plant List) }\end{array}$ \\
\hline Opuntia pilifera F.A.C. Weber & Barcelona & 1964 & 1971 & \\
\hline \multirow[t]{2}{*}{ Opuntia polyacantha Haw. } & Edmonton & $\begin{array}{l}1968 \& 1969 \\
\& 1972\end{array}$ & $\begin{array}{l}1989 \& 1975 \& \text { \& } \\
1979\end{array}$ & 6 synonyms \\
\hline & nn & $\begin{array}{l}\text { in collection } \\
\text { in } 1968\end{array}$ & 1975 & arrived as $O$. rhodantha \\
\hline \multirow[t]{2}{*}{ Opuntia pseudo-tuna Salm-Dyck } & Jalta & $1958 \& 1961$ & $1961 \& 1963$ & \\
\hline & Jalta & $\begin{array}{l}\text { in collection } \\
\text { in } 1963\end{array}$ & 1979 & \\
\hline Opuntia robusta J.C. Wendl. & nn & unknown & 1963 & 4 synonyms \\
\hline Opuntia scheeri F.A.C. Weber & Barcelona & 1961 & 1961 & \\
\hline $\begin{array}{l}\text { Opuntia spirocentra Engelm. \& J.M.Bigelow ex } \\
\text { Haage }\end{array}$ & Tuebingen & 1953 & 1977 & \\
\hline Opuntia stenopetala Engelm. & nn & 1952 & 1961 & $\begin{array}{l}\text { arrived as } O \text {. grandis; from } \\
\text { cuttings }\end{array}$ \\
\hline \multirow[t]{5}{*}{ Opuntia stricta (Haw.) Haw. } & Lausanne & 1954 & no data & $\begin{array}{l}\text { arrived as } O . \text { macrarthra; } \\
\text { one of } 5+\text { synonyms }\end{array}$ \\
\hline & Besancon & 1959 & 1959 & \\
\hline & nn & 1951 & 1961 & $\begin{array}{l}\text { arrived as } O \text {. inermis; from } \\
\text { cuttings }\end{array}$ \\
\hline & Bonn & 1981 & 1982 & arrived as $O$. dillenii \\
\hline & Berlin & 1985 & 2003 & \\
\hline \multirow[t]{2}{*}{ Opuntia sulphurea Gillies ex Salm-Dyck } & Barcelona & 1964 & 1993 & 2 synonyms \\
\hline & Berlin & 1987 & 2003 & \\
\hline Opuntia tomentosa Salm-Dyck & Barcelona & 1968 & 1981 & $\begin{array}{l}\text { arrived as } O . \text { macdougali- } \\
\text { ana; one of } 6 \text { synonyms }\end{array}$ \\
\hline
\end{tabular}




\begin{tabular}{|c|c|c|c|c|}
\hline Scientific name & $\begin{array}{l}\text { City of plant } \\
\text { material origin }\end{array}$ & $\begin{array}{l}\text { Year of } \\
\text { ordering/ } \\
\text { purchasing }\end{array}$ & $\begin{array}{l}\text { Year when plant } \\
\text { was last recorded } \\
\text { in collection }\end{array}$ & Notes and remarks \\
\hline & $\mathrm{nn}$ & unknown & 1963 & arrived as $O$. tomentosa \\
\hline & Mexico & 1990 & 2003 & \\
\hline Opuntia tuna (L.) Mill. & Genova & 1960 & 1968 & 6 synonyms \\
\hline Opuntia winteriana A.Berger & Barcelona & 1964 & 1989 & \\
\hline Opuntia zacuapanensis A.Berger & Antwerpen & 1971 & 1982 & \\
\hline \multirow[t]{4}{*}{ Oreocereus doelzianus (Backeb.) Borg } & Halle & 1973 & 1978 & $\begin{array}{l}\text { arrived as Morawetzia } \\
\text { doelziana; one of } 6 \\
\text { synonyms }\end{array}$ \\
\hline & Esperance & 1970 & 1977 & \\
\hline & $\begin{array}{l}\text { Vienna-Belve- } \\
\text { dere }\end{array}$ & 1971 & 1990 & \\
\hline & Barcelona & 1980 & 2006 & \\
\hline Oreocereus hempelianus (Gürke) D.R. Hunt & Liege & 1973 & 1990 & $\begin{array}{l}\text { arrived as Arequipa } \\
\text { erectocylindrica; } 15+ \\
\text { synonyms }\end{array}$ \\
\hline Oreocereus pseudofossulatus D.R. Hunt & Barcelona & $1971 \& 1980$ & $1993 \& 1989$ & $\begin{array}{l}\text { arrived as } O \text {. fossulatus; } \\
\text { one of } 4 \text { synonyms }\end{array}$ \\
\hline \multirow[t]{5}{*}{ Oreocereus trollii (Kupper) Backeb. } & Rouen & 1956 & 1990 & 5 synonyms \\
\hline & nn & unknown & 1968 & \\
\hline & $\begin{array}{l}\text { Vienna-Belve- } \\
\text { dere }\end{array}$ & 1971 & 1983 & \\
\hline & Halle & 1973 & 1983 & \\
\hline & nn & $\begin{array}{l}\text { in collection } \\
\text { in } 1961\end{array}$ & 1961 & arrived as Cereus trollii \\
\hline Oreocereus variicolor Backeb. & Barcelona & 1980 & 1983 & 4 synonyms \\
\hline Oroya peruviana (K. Schum.) Britton \& Rose & $\begin{array}{l}\text { Vienna-Belve- } \\
\text { dere }\end{array}$ & 1971 & 1983 & $\begin{array}{l}\text { arrived as O. gibbosa; one } \\
\text { of } 5+\text { synonyms }\end{array}$ \\
\hline Pachycereus marginatus (DC.) Britton \& Rose & Beograd & 1965 & 1982 & $\begin{array}{l}\text { arrived as Marginatocereus } \\
\text { marginatus; one of } 5 \\
\text { synonyms }\end{array}$ \\
\hline Parodia aureicentra Backeb. & Monaco & 2003 & 2010 & arrived as $P$. rauschii \\
\hline \multirow[t]{5}{*}{ Parodia ayopayana Cárdenas } & Linz & 1968 & 1972 & 4 synonyms \\
\hline & Muenchen & 1972 & 1977 & \\
\hline & Monaco & 1998 & no data & \\
\hline & Berlin & 1968 & 1978 & arrived as $P$. comosa \\
\hline & Goettingen & 1985 & 1985 & arrived as $P$. echinus \\
\hline Parodia comarapana Cárdenas & Linz & 1965 & 2000 & $\begin{array}{l}\text { arrived as } P \text {. mairanana; } \\
\text { one of } 2 \text { synonyms }\end{array}$ \\
\hline \multirow[t]{12}{*}{ Parodia concinna (Monv.) N.P. Taylor } & Hamburg & $1955 \& 1957$ & $1968 \& 1961$ & $\begin{array}{l}\text { arrived as Notocactus } \\
\text { apricus; one of 5+ } \\
\text { synonyms }\end{array}$ \\
\hline & Copenhagen & 1961 & 1962 & \\
\hline & Berlin & 1965 & 1978 & \\
\hline & Meinz & 1966 & 1983 & \\
\hline & Linz & $1972 \& 1973$ & $1983 \& 1977$ & \\
\hline & Berlin & $\begin{array}{l}\text { in collection } \\
\text { in } 1975\end{array}$ & 1979 & \\
\hline & Goettingen & 1985 & 1990 & \\
\hline & Berlin & $1964 \& 1968$ & 1968 \& 1982 & $\begin{array}{l}\text { arrived as Notocactus } \\
\text { caespitosus }\end{array}$ \\
\hline & Erlangen & 1965 & 1982 & $\begin{array}{l}\text { arrived as Notocactus } \\
\text { concinnus }\end{array}$ \\
\hline & Meinz & 1965 & 1971 & \\
\hline & Graz & 1972 & 1977 & \\
\hline & Gent & 1970 & 1983 & \\
\hline Parodia crassigibba (F. Ritter) N.P. Taylor & Linz & 1975 & 1982 & $\begin{array}{l}\text { arrived as Notocactus } \\
\text { crassigibbus }\end{array}$ \\
\hline Parodia erinacea (Haw.) N.P. Taylor & Berlin & $1955 \& 1958$ & $1975 \& 1961$ & $\begin{array}{l}\text { arrived as Wigginsia } \\
\text { erinacea; one of } 10+ \\
\text { synonyms }\end{array}$ \\
\hline \multirow[t]{3}{*}{ Parodia leninghausii (K. Schum.) F.H. Brandt } & Gent & 1965 & 1975 & $\begin{array}{l}\text { arrived as Eriocactus } \\
\text { lenninghausii }\end{array}$ \\
\hline & Vienna & $1965 \& 1968$ & $1993 \& 1982$ & \\
\hline & Karlsruhe & 1965 & 1977 & \\
\hline
\end{tabular}


S42 Sandev, D. et al.: Plethora of plants - collections of the Botanical Garden, Faculty of Science, University of Zagreb (2)

\begin{tabular}{|c|c|c|c|c|}
\hline Scientific name & $\begin{array}{l}\text { City of plant } \\
\text { material origin }\end{array}$ & $\begin{array}{l}\text { Year of } \\
\text { ordering/ } \\
\text { purchasing }\end{array}$ & $\begin{array}{l}\text { Year when plant } \\
\text { was last recorded } \\
\text { in collection }\end{array}$ & Notes and remarks \\
\hline & nn & 1990 & 1993 & gift from prof. Štimac \\
\hline Parodia maassii (Heese) A. Berger & Berlin & 1965 & $1983 \& 1989$ & $10+$ synonyms \\
\hline Parodia magnifica (F. Ritter) F.H. Brandt & Linz & 1972 & 1980 & $\begin{array}{l}\text { arrived as Eriocactus } \\
\text { magnificus }\end{array}$ \\
\hline \multirow[t]{14}{*}{ Parodia mammulosa (Lem.) N.P. Taylor } & Linz & $1958 \& 1970$ & $1977 \& 1982$ & $\begin{array}{l}\text { arrived as Notocactus } \\
\text { floricomus; on of } 5+ \\
\text { synonyms }\end{array}$ \\
\hline & Berlin & 1965 & 1978 & \\
\hline & Nantes & 1975 & 1983 & \\
\hline & Debrecen & 1980 & 1983 & \\
\hline & Hamburg & 1955 & 1968 & $\begin{array}{l}\text { arrived as Notocactus mam- } \\
\text { mulosus }\end{array}$ \\
\hline & Berlin & $1962 \& 1974$ & $1977 \& 1982$ & \\
\hline & Meinz & 1964 & 1982 & \\
\hline & Delft & 1962 & 1971 & \\
\hline & Blanes & 1987 & 1990 & \\
\hline & Linz & $1972 \& 1987$ & $1983 \& 2003$ & \\
\hline & Jibou & 1989 & 2003 & \\
\hline & Cluj-Napoca & 2000 & 2003 & \\
\hline & Monaco & 2000 & 2003 & \\
\hline & Nantes & 2000 & 2003 & \\
\hline $\begin{array}{l}\text { Parodia mammulosa subsp. eugeniae (Vliet) } \\
\text { Hofacker }\end{array}$ & Goettingen & 1985 & 1990 & $\begin{array}{l}\text { arrived as Notocactus } \\
\text { eugeniae; one of } 2 \\
\text { synonyms }\end{array}$ \\
\hline \multirow[t]{8}{*}{$\begin{array}{l}\text { Parodia mammulosa subsp. submammulosus (Lem.) } \\
\text { Hofacker }\end{array}$} & Berlin & 1965 & 1982 & $\begin{array}{l}\text { arrived as Notocactus } \\
\text { submammulosus; one of } 4 \\
\text { synonyms }\end{array}$ \\
\hline & Linz & 1973 & 1974 & \\
\hline & Wageningen & 1972 & 1979 & \\
\hline & Utrecht & $\begin{array}{l}1952 \& 1954 \\
\& 1964\end{array}$ & $\begin{array}{l}1961 \& 1975 \& \\
\text { no data }\end{array}$ & $\begin{array}{l}\text { arrived as Echinocactus } \\
\text { submammulosus }\end{array}$ \\
\hline & Berlin & 1979 & 1983 & $\begin{array}{l}\text { arrived as Notocactus } \\
\text { submammulosus var. } \\
\text { pampeanus }\end{array}$ \\
\hline & Berlin & 1955 & unknown & $\begin{array}{l}\text { arrived as Notocactus } \\
\text { pampeanus; one of } 4 \\
\text { synonyms }\end{array}$ \\
\hline & Zagreb & 1966 & 1983 & \\
\hline & Wageningen & 1972 & 1982 & \\
\hline \multirow[t]{14}{*}{$\begin{array}{l}\text { Parodia mueller-melchersii (Frič ex Backeb.) N.P. } \\
\text { Taylor }\end{array}$} & Berlin & $1965 \& 1968$ & $1978 \& 1978$ & $\begin{array}{l}\text { arrived as Notocactus } \\
\text { rutilans; one of } 6 \\
\text { synonyms }\end{array}$ \\
\hline & Linz & 1966 & 1982 & \\
\hline & Hamburg & 1968 & 1982 & \\
\hline & Nantes & 1965 & 1975 & \\
\hline & Goettingen & 1985 & 1990 & \\
\hline & Vienna & 1987 & 1990 & \\
\hline & Jibou & 1996 & 2000 & \\
\hline & Linz & $\begin{array}{l}1971 \& 1972 \\
\& 1973 \& \\
1975\end{array}$ & $\begin{array}{l}1982 \& 1980 \& \\
1983 \& 1982\end{array}$ & \begin{tabular}{|l|} 
arrived as Notocactus \\
mueller-melchersii; one of 4 \\
synonyms
\end{tabular} \\
\hline & Berlin & 1971 & 1975 & \\
\hline & Nantes & 1970 & 1982 & \\
\hline & Gent & 1967 & 1982 & \\
\hline & nn & 1962 & 1982 & $\begin{array}{l}\text { arrived as P. mueller- } \\
\text { melchersii subsp. } \\
\text { mueller-melchersii var. } \\
\text { gracilispina }\end{array}$ \\
\hline & Nantes & 1973 & 1979 & \\
\hline & Linz & 1973 & 1974 & \\
\hline Parodia muricata (Otto) Hofacker & Berlin & $1965 \& 1968$ & $1989 \& 1983$ & $\begin{array}{l}\text { arrived as Notocactus } \\
\text { muricatus; one of } 3 \\
\text { synonyms }\end{array}$ \\
\hline \multirow[t]{2}{*}{ Parodia mutabilis Backeb. } & Vienna & 1953 & 1961 & $\begin{array}{l}\text { unresolved name (The } \\
\text { Plant List); } 2 \text { synonyms }\end{array}$ \\
\hline & Berlin & 1964 & 1966 & \\
\hline
\end{tabular}




\begin{tabular}{|c|c|c|c|c|}
\hline Scientific name & $\begin{array}{l}\text { City of plant } \\
\text { material origin }\end{array}$ & $\begin{array}{l}\text { Year of } \\
\text { ordering/ } \\
\text { purchasing }\end{array}$ & $\begin{array}{l}\text { Year when plant } \\
\text { was last recorded } \\
\text { in collection }\end{array}$ & Notes and remarks \\
\hline & Lokrum & $1967 \& 1969$ & $1982 \& 1975$ & \\
\hline Parodia nothorauschii D.R. Hunt & Wageningen & 1974 & 1982 & $\begin{array}{l}\text { arrived as Notocactus } \\
\text { rauschii; one of } 2 \\
\text { synonyms }\end{array}$ \\
\hline Parodia ocampoi Cárdenas & Debrecen & 1980 & 1990 & arrived as $P$. punae \\
\hline \multirow[t]{12}{*}{ Parodia ottonis (Lehm.) N.P. Taylor } & Linz & 1972 & 1978 & $\begin{array}{l}\text { arrived as Notocactus } \\
\text { acutus; one of } 10+ \\
\text { synonyms }\end{array}$ \\
\hline & Jibou & 1989 & 1993 & \\
\hline & nn & 1952 & 1979 & $\begin{array}{l}\text { arrived as Notocactus } \\
\text { ottonis, from cuttings }\end{array}$ \\
\hline & Meinz & 1964 & 1971 & \\
\hline & Nantes & 1966 & 1975 & \\
\hline & Berlin & 1971 & 1979 & \\
\hline & Budapest & 1978 & 1990 & \\
\hline & Cluj-Napoca & 1979 & no data & \\
\hline & Liege & 1984 & 2003 & \\
\hline & Linz & 1967 & 1983 & $\begin{array}{l}\text { arrived as } P \text {. ottonis } \\
\text { rubrispinus }\end{array}$ \\
\hline & Berlin & 1979 & 1981 & $\begin{array}{l}\text { arrived as P. ottonis v. } \\
\text { tortuosus }\end{array}$ \\
\hline & Budapest & 1978 & 1983 & $\begin{array}{l}\text { arrived as } P \text {. ottonis var. } \\
\text { paraguayensis }\end{array}$ \\
\hline Parodia procera F. Ritter & Debrecen & 1980 & 1983 & arrived $P$. gracilis \\
\hline Parodia ritteri Buining & Linz & 1966 & 1968 & arrived as $P$. carrerana \\
\hline Parodia sanagasta Frič ex Weing. & Linz & 1965 & 1978 & $\begin{array}{l}\text { syn. P. microsperma var. } \\
\text { sanagasta }\end{array}$ \\
\hline \multirow[t]{2}{*}{ Parodia schumanniana (Nicolai) F.H. Brandt } & Meinz & 1967 & 1980 & $\begin{array}{l}\text { arrived as Eriocactus } \\
\text { schumannianus; one of } 5+ \\
\text { synonyms }\end{array}$ \\
\hline & Linz & 1987 & 2000 & \\
\hline Parodia scopa (Spreng.) N.P. Taylor subsp. scopa & Berlin & 1955 & no data & $\begin{array}{l}\text { arrived as Notocactus } \\
\text { scopa, one of } 4 \text { synonyms }\end{array}$ \\
\hline \multirow[t]{12}{*}{ Parodia sellowii (Link \& Otto) D.R. Hunt } & Berlin & 1979 & 1983 & $\begin{array}{l}\text { arrived as Notocactus } \\
\text { corynodes; one of } 10+ \\
\text { synonyms }\end{array}$ \\
\hline & Berlin & 1979 & 1981 & arrived as Notocactus fricii \\
\hline & Berlin & 1965 & 1968 & arrived as Wigginsia fricii \\
\hline & Nantes & 1970 & 1980 & \\
\hline & Linz & $1967 \& 1970$ & $1980 \& 1979$ & $\begin{array}{l}\text { arrived as Wigginsia } \\
\text { sessiliflora }\end{array}$ \\
\hline & Linz & 1969 & 1983 & $\begin{array}{l}\text { arrived as Wigginsia } \\
\text { tephracantha var. courantii }\end{array}$ \\
\hline & Berlin & $1965 \& 1967$ & $1971 \& 1979$ & $\begin{array}{l}\text { arrived as Wigginsia } \\
\text { vorwerkiana }\end{array}$ \\
\hline & Berlin & 1968 & 1983 & $\begin{array}{l}\text { arrived as Wigginsia } \\
\text { corynodes }\end{array}$ \\
\hline & Linz & 1971 & 1983 & \\
\hline & Monaco & 1986 & 1993 & \\
\hline & Dresden & 1975 & 1983 & $\begin{array}{l}\text { arrived as Wigginsia } \\
\text { tephracantha }\end{array}$ \\
\hline & Saarbrucken & 1981 & 1993 & \\
\hline \multirow[t]{5}{*}{ Parodia tabularis (F. Cels ex Rumpler) D.R. Hunt } & Frankfurt & 1959 & 1989 & $\begin{array}{l}\text { arrived as Notocactus } \\
\text { tabularis; one of } 5 \\
\text { synonyms }\end{array}$ \\
\hline & Muenchen & 1964 & 1989 & \\
\hline & Berlin & 1965 & 1989 & \\
\hline & Linz & 1972 & 1990 & \\
\hline & Halle & 1979 & 1983 & \\
\hline Parodia tenuicylindrica (F. Ritter) D.R. Hunt & Debrecen & 1980 & 1981 & $\begin{array}{l}\text { arrived as Notocactus } \\
\text { minimus }\end{array}$ \\
\hline Parodia tilcarensis (Werderm. \& Backeb.) Backeb. & Berlin & 1965 & 1990 & arrived as $P$. carminata \\
\hline \multirow[t]{2}{*}{ Parodia werdermanniana (Herter) N.P. Taylor } & Linz & $1972 \& 1973$ & $1983 \& 1974$ & $\begin{array}{l}\text { arrived as Notocactus } \\
\text { vanvlietii }\end{array}$ \\
\hline & Berlin & 1979 & 1983 & \\
\hline
\end{tabular}


S44 Sandev, D. et al.: Plethora of plants - collections of the Botanical Garden, Faculty of Science, University of Zagreb (2)

\begin{tabular}{|c|c|c|c|c|}
\hline Scientific name & $\begin{array}{l}\text { City of plant } \\
\text { material origin }\end{array}$ & $\begin{array}{l}\text { Year of } \\
\text { ordering/ } \\
\text { purchasing }\end{array}$ & $\begin{array}{l}\text { Year when plant } \\
\text { was last recorded } \\
\text { in collection }\end{array}$ & Notes and remarks \\
\hline Parodia werneri Hofacker & Goettingen & 1985 & 1990 & $\begin{array}{l}\text { arrived as Notocactus } \\
\text { arachnites }\end{array}$ \\
\hline Pereskiopsis diguetii (F.A.C. Weber) Britton \& Rose & Krakow & 1970 & 1983 & $\begin{array}{l}\text { arrived as } P \text {. spathulata; } \\
\text { one of } 3 \text { synonyms }\end{array}$ \\
\hline Phyllocactus $\times$ hybridus Voss & nn & $\begin{array}{l}\text { in collection } \\
\text { in } 1961\end{array}$ & 1961 & $\begin{array}{l}\text { arrived as Phyllocactus } \\
\text { hybridus hort. }\end{array}$ \\
\hline Pilosocereus arrabidae (Lem.) Byles \& G.D. Rowley & Adelaide & $1971 \& 1972$ & $1980 \& 1974$ & 4 synonyms \\
\hline \multirow[t]{2}{*}{ Pilosocereus brasiliensis (Britton \& Rose) Backeb. } & nn & 1953 & no data & arrived as P. sublanatus \\
\hline & Lisbon & 1954 & 1961 & \\
\hline \multirow[t]{2}{*}{ Pilosocereus coerulescens (Lem.) F.Ritter } & Halle & 1956 \& 1958 & $1977 \& 1993$ & $\begin{array}{l}\text { arrived as Cereus } \\
\text { coerulescens; } 6 \text { synonyms; } \\
\text { unresolved name (The } \\
\text { Plant List) }\end{array}$ \\
\hline & Berlin & 1987 & 1990 & \\
\hline \multirow[t]{7}{*}{$\begin{array}{l}\text { Pilosocereus leucocephalus (Poselg.) Byles \& G.D. } \\
\text { Rowley }\end{array}$} & Barcelona & 1964 & 1968 & $\begin{array}{l}\text { arrived as Cereus cometes; } \\
\text { one of } 5+\text { synonyms }\end{array}$ \\
\hline & Nancy & 1966 & 1971 & arrived as P. leucocephalus \\
\hline & Freiburg & 1956 & 1975 & arrived as $P$. sartorianus \\
\hline & Cluj-Napoca & 1993 & 1997 & \\
\hline & nn & unknown & 1971 & arrived as P. palmeri \\
\hline & $\begin{array}{l}\text { Vienna- Belve- } \\
\text { dere }\end{array}$ & 1993 & 2000 & \\
\hline & Muenchen & 1993 & 1993 & \\
\hline \multirow[t]{2}{*}{ Polaskia chichipe (Gosselin) Backeb. } & Halle & 1973 & 2000 & 5 synonyms \\
\hline & Monaco & 1997 & 2000 & \\
\hline $\begin{array}{l}\text { Pyrrhocactus robustus F. Ritter var. vegasanus } \\
\text { F.Ritter }\end{array}$ & Linz & 1964 & 1967 & \\
\hline \multirow[t]{2}{*}{ Rebutia aureiflora Backeb. } & Berlin & 1965 & 1990 & $\begin{array}{l}\text { arrived as Mediolobivia } \\
\text { euanthema var.fričii }\end{array}$ \\
\hline & Karlsruhe & $1963 \& 1969$ & 1990 \& no data & $\begin{array}{l}\text { arrived as Mediolobivia } \\
\text { aureiflora }\end{array}$ \\
\hline \multirow[t]{5}{*}{ Rebutia deminuta (F.A.C. Weber) Britton \& Rose } & Halle & 1955 & unknown & $\begin{array}{l}\text { arrived as R. pseudodem- } \\
\text { inuta; one of } 10+ \\
\text { synonyms }\end{array}$ \\
\hline & Basel & $1957 \& 1965$ & $1971 \& 1979$ & \\
\hline & Basel & $1957 \& 1965$ & $1972 \& 1979$ & $\begin{array}{l}\text { arrived as Aylostera } \\
\text { deminuta }\end{array}$ \\
\hline & nn & $\begin{array}{l}\text { in collection } \\
\text { in } 1970\end{array}$ & 1981 & \\
\hline & Kiev & 1989 & 1993 & \\
\hline Rebutia einsteinii Frič & Gent & 1965 & 1975 & $\begin{array}{l}\text { arrived as Mediolobivia } \\
\text { neopygmaea }\end{array}$ \\
\hline \multirow[t]{12}{*}{ Rebutia marsoneri Werderm. } & Linz & 1964 & 1983 & $\begin{array}{l}\text { arrived as R. hyalacantha; } \\
\text { one of } 3 \text { synonyms }\end{array}$ \\
\hline & Gent & 1967 & 1978 & \\
\hline & Košice & 1978 & 1983 & \\
\hline & Goettingen & 1989 & 1990 & \\
\hline & Halle & $\begin{array}{l}1965 \& 1967 \\
\& 1968\end{array}$ & $\begin{array}{l}1979 \& 1990 \& \\
1989\end{array}$ & arrived as $R$. krainziana \\
\hline & Gent & 1968 & 1989 & \\
\hline & Dresden & 1986 & 1990 & \\
\hline & Halle & $\begin{array}{l}1961 \& 1963 \\
\& 1965\end{array}$ & $\begin{array}{l}1968 \& 1966 \& \\
1983\end{array}$ & arrived as $R$. marsoneri \\
\hline & Nantes & 1965 & 1982 & \\
\hline & Gent & 1965 & 1981 & \\
\hline & nn & unknown & 1968 & \\
\hline & Wageningen & 1980 & 1983 & \\
\hline \multirow[t]{6}{*}{ Rebutia minuscula K. Schum. } & Gent & 1964 & 1983 & $\begin{array}{l}\text { arrived as } R \text {. chrysacantha; } \\
\text { one of } 20+\text { synonyms }\end{array}$ \\
\hline & Nantes & 1966 & 1971 & \\
\hline & Turku & 1967 & 1978 & \\
\hline & Rouen & 1962 & 1971 & arrived as R. grandiflora \\
\hline & Gent & 1966 & 1979 & \\
\hline & Budapest & 1970 & 1979 & \\
\hline
\end{tabular}




\begin{tabular}{|c|c|c|c|c|}
\hline Scientific name & $\begin{array}{l}\text { City of plant } \\
\text { material origin }\end{array}$ & $\begin{array}{l}\text { Year of } \\
\text { ordering/ } \\
\text { purchasing }\end{array}$ & $\begin{array}{l}\text { Year when plant } \\
\text { was last recorded } \\
\text { in collection }\end{array}$ & Notes and remarks \\
\hline & Goettingen & 1985 & & \\
\hline & Leipzig & 1958 & 1966 & arrived as $R$. violaciflora \\
\hline & Basel & $1961 \& 1967$ & $1978 \& 1975$ & \\
\hline & Cluj-Napoca & 1979 & 1983 & \\
\hline & Vienna & 1992 & 1997 & \\
\hline & Bratislava & 2000 & 2003 & \\
\hline & Graz & 1975 & 1983 & $\begin{array}{l}\text { arrived as R. senilis (var. } \\
\text { kesselringiana) }\end{array}$ \\
\hline & Rouen & 1955 & 1982 & $\begin{array}{l}\text { arrived as R. xanthocarpa } \\
\text { var.citricarpa }\end{array}$ \\
\hline & $\operatorname{Linz}$ & 1970 & 1979 & $\begin{array}{l}\text { arrived as R.xanthocarpa } \\
\text { var. dasyphrissa }\end{array}$ \\
\hline & Rouen & 1966 & 1975 & \\
\hline & Berlin & 1971 & 1979 & \\
\hline & nn & 1949 & no data & $\begin{array}{l}\text { arrived as R. xanthocarpa } \\
\text { var. salmonea }\end{array}$ \\
\hline & nn & $\begin{array}{l}\text { in collection } \\
\text { in } 1961\end{array}$ & 1968 & \\
\hline & Wageningen & 1974 & 1978 & $\begin{array}{l}\text { arrived as R. senilis var. } \\
\text { lilacinorosea }\end{array}$ \\
\hline & nn & 1975 & 1982 & arrived as R. minuscula \\
\hline & Dresden & 1975 & 1989 & \\
\hline & Berlin & $1958 \& 1971$ & 1978 \& 1982 & arrived as R. senilis \\
\hline & $\operatorname{Lin} z$ & 1970 & 1983 & \\
\hline & Wageningen & $\begin{array}{l}1972 \& 1974 \\
\& 1980\end{array}$ & $\begin{array}{l}1978 \& 1983 \& \\
1981\end{array}$ & \\
\hline & Jibou & 1984 & 1990 & \\
\hline & Liege & 1984 & 1989 & \\
\hline & Goettingen & 1985 & 1990 & \\
\hline & Bratislava & 2000 & 2003 & \\
\hline & Gent & 1962 & 1975 & $\begin{array}{l}\text { arrived as R. minuscula } \\
\text { var. grandiflora }\end{array}$ \\
\hline $\begin{array}{l}\text { Rebutia minuscula var. wessneriana (Bewer.) } \\
\text { Eb.Scholz }\end{array}$ & Madrid & 1997 & 2000 & $\begin{array}{l}\text { arrived as } R \text {. wessneriana; } \\
\text { one of } 4 \text { synonyms }\end{array}$ \\
\hline Rebutia pygmaea (R.E. Fr.) Britton \& Rose & Debrecen & 1980 & 1989 & $\begin{array}{l}\text { arrived as Mediolobivia } \\
\text { haefneriana }\end{array}$ \\
\hline Rebutia ritteri (Wessner) F. Ritter & Vienna & 1972 & 1982 & $\begin{array}{l}\text { arrived as Mediolobivia } \\
\text { ritteri; one of } 3 \text { synonyms }\end{array}$ \\
\hline Rebutia steinmannii (Solms) Britton \& Rose & Saarbrucken & 1968 & 1982 & $\begin{array}{l}\text { arrived as Mediolobivia } \\
\text { brachyantha }\end{array}$ \\
\hline \multirow[t]{2}{*}{$\begin{array}{l}\text { Rebutia violaciflora Backeb. var. knuthiana } \\
\text { (Backeb.) Donald }\end{array}$} & Debrecen & 1980 & 1983 & \\
\hline & Essen & 1985 & 1990 & \\
\hline Rebutia wessneriana Bewer. & Karlsruhe & 1967 & 1990 & $\begin{array}{l}\text { arrived as } R \text {. calliantha; } \\
\text { one of } 2 \text { synonyms }\end{array}$ \\
\hline \multirow[t]{9}{*}{ Rhipsalis baccifera (Sol.) Stearn } & Koeln & 1960 & 1963 & $\begin{array}{l}\text { arrived as } R \text {. cereuscula; } \\
\text { one of } 5+\text { synonyms }\end{array}$ \\
\hline & Muenster & 1964 & 1964 & \\
\hline & Goettingen & 1966 & 1971 & \\
\hline & Essen & 1960 & 1978 & arrived as $R$. fasciculata \\
\hline & Muenster & 1964 & 1964 & \\
\hline & Stocholm & 1960 & 1971 & arrived as $R$. prismatica \\
\hline & Antwerpen & $1965 \& 1971$ & $1975 \& 1975$ & \\
\hline & Goettingen & 1966 & 1966 & \\
\hline & nn & 1954 & 1980 & $\begin{array}{l}\text { arrived as R. suareziana; } \\
\text { from cuttings }\end{array}$ \\
\hline $\begin{array}{l}\text { Rhipsalis baccifera subsp. shaferi (Britton \& Rose) } \\
\text { Barthlott \& N.P.Taylor }\end{array}$ & Goettingen & 1966 & 1980 & arrived as $R$. shaferi \\
\hline \multirow[t]{2}{*}{ Rhipsalis campos-portoana Loefgr. } & Bergen & 1960 & 1968 & $\begin{array}{l}\text { syn. Erythrorhipsalis } \\
\text { campos-portoana }\end{array}$ \\
\hline & Antwerpen & $1960 \& 1961$ & $1968 \& 1971$ & \\
\hline Rhipsalis clavata F.A.C. Weber & Goettingen & 1966 & 1990 & arrived as Hatiora clavata \\
\hline Rhipsalis dissimilis (G. Lindb.) K. Schum. & Muenster & 1965 & 1975 & $\begin{array}{l}\text { arrived as Lepismium } \\
\text { chrysanthum; one of } 4 \\
\text { synonyms }\end{array}$ \\
\hline
\end{tabular}


S46 Sandev, D. et al.: Plethora of plants - collections of the Botanical Garden, Faculty of Science, University of Zagreb (2)

\begin{tabular}{|c|c|c|c|c|}
\hline Scientific name & $\begin{array}{l}\text { City of plant } \\
\text { material origin }\end{array}$ & $\begin{array}{l}\text { Year of } \\
\text { ordering/ } \\
\text { purchasing }\end{array}$ & $\begin{array}{l}\text { Year when plant } \\
\text { was last recorded } \\
\text { in collection }\end{array}$ & Notes and remarks \\
\hline & Muenster & 1964 & 1984 & $\begin{array}{l}\text { arrived as Lepismium } \\
\text { dissimile }\end{array}$ \\
\hline & $\mathrm{nn}$ & 1954 & 1977 & from cuttings \\
\hline \multirow{2}{*}{ Rhipsalis elliptica G.Lindb. ex K.Schum. } & Goettingen & 1966 & 1977 & \\
\hline & Szeged & 1970 & 1971 & \\
\hline \multirow[t]{2}{*}{$\begin{array}{l}\text { Rhipsalis floccosa Salm-Dyck ex Pfeiff. subsp. } \\
\text { pulvinigera (G. Lindb.) Barthlott \& N.P. Taylor }\end{array}$} & nn & $\begin{array}{l}\text { in collection } \\
\text { in } 1961\end{array}$ & 1981 & $\begin{array}{l}\text { arrived as R. pulvinigera; } \\
\text { one of } 4 \text { synonyms; from } \\
\text { cuttings }\end{array}$ \\
\hline & Duisburg & 1961 & 1981 & arrived as R. gibberula \\
\hline \multirow[t]{4}{*}{ Rhipsalis grandiflora Haw. } & Vacratot & $1961 \& 1965$ & 2000 \& no data & 5 synonyms \\
\hline & nn & $\begin{array}{l}\text { in collection } \\
\text { in } 1974\end{array}$ & 1989 & \\
\hline & Antwerpen & 1971 & 1979 & \\
\hline & Dresden & 1956 & 1977 & $\begin{array}{l}\text { arrived as } R . \text { hadrosoma; } \\
\text { one of } 2 \text { synonyms }\end{array}$ \\
\hline \multirow[t]{3}{*}{ Rhipsalis mesembryanthemoides Haw. } & Antwerpen & $1955 \& 1961$ & $1966 \& 1978$ & \\
\hline & Muenster & 1964 & 1971 & \\
\hline & Szeged & 1983 & unknown & \\
\hline Rhipsalis micrantha (Kunth) DC. & Antwerpen & 1965 & 1979 & 5 synonyms \\
\hline Rhipsalis neves-armondii K. Schum. & Muenster & $1964 \& 1968$ & $1964 \& 1978$ & $\begin{array}{l}\text { arrived as Lepismium } \\
\text { neves-armondii }\end{array}$ \\
\hline Rhipsalis pachyptera Pfeiff. & Vacratot & 1961 & 1975 & 2 synonyms \\
\hline \multirow[t]{3}{*}{$\begin{array}{l}\text { Rhipsalis paradoxa (Salm-Dyck ex Pfeiff.) Salm- } \\
\text { Dyck }\end{array}$} & nn & $1953 \& 1954$ & no data & 3 synonyms \\
\hline & nn & $\begin{array}{l}\text { in collection } \\
\text { in } 1963\end{array}$ & 1991 & \\
\hline & Antwerpen & 1971 & 1981 & \\
\hline \multirow[t]{2}{*}{ Rhipsalis pentaptera Pfeiff. ex A. Dietr. } & nn & 1954 & 1961 & $\begin{array}{l}\text { syn. Hatiora pentaptera; } \\
\text { from cuttings }\end{array}$ \\
\hline & $\mathrm{nn}$ & $\begin{array}{l}\text { in collection } \\
\text { in } 1963\end{array}$ & 1980 & \\
\hline \multirow[t]{3}{*}{ Rhipsalis pilocarpa Loefgr. } & Karlsruhe & 1962 & 1971 & $\begin{array}{l}\text { arrived as Erythrorhipsalis } \\
\text { pilocarpa }\end{array}$ \\
\hline & Muenster & 1964 & 1966 & \\
\hline & Goettingen & 1966 & 1977 & \\
\hline Rhipsalis pittieri Britton \& Rose & Goettingen & 1966 & 1980 & $\begin{array}{l}\text { arrived as Lepismium } \\
\text { pittieri; one of } 2 \text { synonyms }\end{array}$ \\
\hline Rhipsalis puniceodiscus G.Lindb. & Gent & 1962 & 1966 & $\begin{array}{l}\text { arrived as Lepismium } \\
\text { punicea-discus; one of } 2 \\
\text { synonyms }\end{array}$ \\
\hline Rhipsalis rhombea (Salm-Dyck) Pfeiff. & nn & 1954 & 1961 & $\begin{array}{l}\text { syn. Cereus rhombeus; from } \\
\text { cuttings }\end{array}$ \\
\hline \multirow[t]{11}{*}{ Rhipsalis teres (Vell.) Steud. } & nn & 1954 & 1961 & $\begin{array}{l}\text { arrived as R. capilliformis; } \\
20+\text { synonyms }\end{array}$ \\
\hline & nn & $\begin{array}{l}\text { in collection } \\
\text { in } 1963\end{array}$ & 1978 & \\
\hline & Krakow & 1971 & 1990 & \\
\hline & Kirstenbosch & 1963 & 1973 & \\
\hline & Antwerpen & 1970 & 1978 & \\
\hline & nn & $\begin{array}{l}\text { unknown \& } \\
\text { in collection } \\
\text { in } 1963\end{array}$ & $1961 \& 1977$ & $\begin{array}{l}\text { arrived as } R \text {. heteroclada; } \\
\text { from cuttings (unknown) }\end{array}$ \\
\hline & Antwerpen & 1971 & 1977 & \\
\hline & Antwerpen & 1955 & 1963 & $\begin{array}{l}\text { arrived as R. teres; from } \\
\text { cuttings }\end{array}$ \\
\hline & Tuebingen & 1971 & 1975 & \\
\hline & Krakow & 1970 & 1979 & \\
\hline & Bucharest & 1985 & 1990 & \\
\hline \multirow[t]{3}{*}{ Rhipsalis trigona Pfeiff. } & Uppsala & 1964 & 1966 & $\begin{array}{l}\text { arrived as Lepismium } \\
\text { trigonum; one of } 2 \\
\text { synonyms }\end{array}$ \\
\hline & Gent & $1962 \& 1963$ & $1964 \& 1966$ & \\
\hline & Tuebingen & 1971 & 1980 & \\
\hline
\end{tabular}




\begin{tabular}{|c|c|c|c|c|}
\hline Scientific name & $\begin{array}{l}\text { City of plant } \\
\text { material origin }\end{array}$ & $\begin{array}{l}\text { Year of } \\
\text { ordering/ } \\
\text { purchasing }\end{array}$ & $\begin{array}{l}\text { Year when plant } \\
\text { was last recorded } \\
\text { in collection }\end{array}$ & Notes and remarks \\
\hline \multirow[t]{2}{*}{ Rhipsalis tucumanensis F.A.C. Weber } & Muenster & $\begin{array}{l}1964 \& 1965 \\
\& 1968\end{array}$ & $\begin{array}{l}1993 \& 1968 \& \\
1968\end{array}$ & $\begin{array}{l}\text { arrived as Lepismium } \\
\text { tucumanense; one of } 4 \\
\text { synonyms }\end{array}$ \\
\hline & Goettingen & 1966 & 1968 & from cuttings \\
\hline Rhipsalis zanzibarica F.A.C.Weber & Muenster & 1964 & 1966 & \\
\hline Ritterocereus hystrix (Haw.) Backeb. & Nantes & 1958 & 1989 & $\begin{array}{l}\text { arrived as Cactus hystrix; } \\
\text { one of } 3 \text { synonyms }\end{array}$ \\
\hline \multirow[t]{2}{*}{ Schlumbergera russelliana (Hook.) Britton \& Rose } & Bergen & 1955 & 1961 & $\begin{array}{l}\text { syn. Epiphyllum } \\
\text { russellianum }\end{array}$ \\
\hline & Vacratot & 1966 & 1971 & \\
\hline $\begin{array}{l}\text { Selenicereus boeckmannii (Otto ex Salm-Dyck) } \\
\text { Britton \& Rose }\end{array}$ & Antwerpen & $1950 \& 1954$ & $1983 \& 1954$ & \begin{tabular}{|l} 
arrived as Cereus \\
boeckmannii; from cuttings \\
$(1950)$
\end{tabular} \\
\hline Selenicereus coniflorus (Weing.) Britton \& Rose & Basel & 1972 & 1993 & $\begin{array}{l}\text { arrived as S. pringlei; one } \\
\text { of } 2 \text { synonyms }\end{array}$ \\
\hline \multirow[t]{10}{*}{ Selenicereus grandiflorus (L.) Britton \& Rose } & nn & $\begin{array}{l}\text { in collection } \\
\text { in 1961 \& in } \\
\text { collection in } \\
1990\end{array}$ & no data \& 2016 & $20+$ synonyms \\
\hline & Gent & $1964 \& 1966$ & $2000 \& 1967$ & \\
\hline & Hamburg & 1966 & 1967 & \\
\hline & Barcelona & 1971 & 1983 & \\
\hline & Liege & 1984 & 1989 & \\
\hline & Bukurešt & 1985 & 1989 & \\
\hline & Wroclaw & 1986 & 1989 & \\
\hline & nn & $\begin{array}{l}\text { in collection } \\
\text { in } 1961\end{array}$ & 1980 & $\begin{array}{l}\text { arrived as Cereus } \\
\text { grandiflorus }\end{array}$ \\
\hline & Poznan & 1966 & 1983 & \\
\hline & Gent & 1964 & 2000 & \\
\hline $\begin{array}{l}\text { Selenicereus grandiflorus subsp. donkelaarii (Salm- } \\
\text { Dyck) Ralf Bauer }\end{array}$ & Basel & 1973 & 2000 & $\begin{array}{l}\text { arrived as } S \text {. donkelaarii; } \\
\text { one of } 2 \text { synonyms }\end{array}$ \\
\hline \multirow[t]{2}{*}{ Selenicereus macdonaldiae (Hook.) Britton \& Rose } & Harkov & 1957 & 1989 & 5 synonyms \\
\hline & Krakow & 1971 & 1975 & \\
\hline Selenicereus nelsonii (Weing.) Britton \& Rose & Berlin & $\begin{array}{l}1963 \& 1965 \\
\& 1969\end{array}$ & $\begin{array}{l}1971 \& 1993 \& \\
1989\end{array}$ & syn. Cereus nelsonii \\
\hline \multirow[t]{2}{*}{ Selenicereus vagans (K. Brandegee) Britton \& Rose } & Berlin & 1967 & 2000 & 5 synonyms \\
\hline & nn & $\begin{array}{l}\text { in collection } \\
\text { in } 1975\end{array}$ & 2000 & \\
\hline \multirow[t]{3}{*}{ Stenocactus crispatus (DC.) A. Berger ex A.W. Hill } & Halle & 1966 & 1990 & $\begin{array}{l}\text { arrived as Echinofossulo- } \\
\text { cactus crispatus; one of } 2 \\
\text { synonyms }\end{array}$ \\
\hline & Nantes & 1970 & 1980 & $\begin{array}{l}\text { arrived as Echinofossulo- } \\
\text { cactus kellerianus }\end{array}$ \\
\hline & Bordeaux & 1992 & 1993 & \\
\hline Stenocereus dumortieri (Scheidw.) Buxb. & Beograd & 1964 & 1993 & 5 synonyms \\
\hline Stenocereus pruinosus (Otto ex Pfeiff.) Buxb. & Copenhagen & 1964 & 1978 & $\begin{array}{l}\text { arrived as Lemaireocereus } \\
\text { longispinus; one of } 3 \\
\text { synonyms }\end{array}$ \\
\hline Stenocereus pruinosus (Otto ex Pfeiff.) Buxb. & Linz & 1966 & 1993 & $\begin{array}{l}\text { arrived as Ritterocereus } \\
\text { laevigatus; one of } 10+\text { syno- } \\
\text { nyms }\end{array}$ \\
\hline \multirow[t]{3}{*}{ Stenocereus queretaroensis (F.A.C. Weber) Buxb. } & $\begin{array}{l}\text { San Marino } \\
\text { (California) }\end{array}$ & 1976 & 1989 & $\begin{array}{l}\text { arrived as Ritterocereus } \\
\text { queretaroensis; one of } 10+ \\
\text { synonyms }\end{array}$ \\
\hline & Esperance & 1970 & 1989 & \\
\hline & Esperance & 1970 & 1983 & $\begin{array}{l}\text { arrived as Lemaireocereus } \\
\text { queretaroensis }\end{array}$ \\
\hline Stenocereus stellatus (Pfeiff.) Riccob. & Debrecen & 1980 & 1993 & 4 synonyms \\
\hline Stephanocereus leucostele (Gürke) A. Berger & Barcelona & 1980 & 1993 & 2 synonyms \\
\hline Stetsonia coryne (Salm-Dyck) Britton \& Rose & Berlin & 1987 & 1990 & $\begin{array}{l}\text { arrived as Cereus coryne; } 2 \\
\text { synonyms }\end{array}$ \\
\hline Stetsonia coryne var. procera F. Ritter & Monaco & 2000 & 2003 & $\begin{array}{l}\text { arrived as C. coryne var. } \\
\text { procera }\end{array}$ \\
\hline
\end{tabular}


S48 Sandev, D. et al.: Plethora of plants - collections of the Botanical Garden, Faculty of Science, University of Zagreb (2)

\begin{tabular}{|c|c|c|c|c|}
\hline Scientific name & $\begin{array}{l}\text { City of plant } \\
\text { material origin }\end{array}$ & $\begin{array}{l}\text { Year of } \\
\text { ordering/ } \\
\text { purchasing }\end{array}$ & $\begin{array}{l}\text { Year when plant } \\
\text { was last recorded } \\
\text { in collection }\end{array}$ & Notes and remarks \\
\hline \multirow[t]{2}{*}{ Sulcorebutia steinbachii (Werderm.) Backeb. } & Halle & 1973 & 1977 & $\begin{array}{l}\text { arrived as Rebutia } \\
\text { steinbachii subsp. } \\
\text { Steinbachii; one of 20+ } \\
\text { synonyms }\end{array}$ \\
\hline & Innsbruck & 2002 & 2010 & \\
\hline $\begin{array}{l}\text { Tacinga palmadora (Britton \& Rose) N.P.Taylor \& } \\
\text { Stuppy }\end{array}$ & Antibes & 1955 & 1963 & $\begin{array}{l}\text { arrived as Opuntia } \\
\text { palmadora }\end{array}$ \\
\hline Tephrocactus articulatus (Pfeiff.) Backeb. & Lokrum & 1969 & 1978 & $\begin{array}{l}\text { arrived as T. syringacan- } \\
\text { thus; one of } 3 \text { synonyms }\end{array}$ \\
\hline Tephrocactus diademata (Lem.) Lem. & Vienna & 1964 & 1990 & syn. Opuntia diademata \\
\hline Tephrocactus inermis (Speg.) Backeb. & Lokrum & 1969 & 1977 & $\begin{array}{l}\text { syn. T. glomeratus var. } \\
\text { inermis }\end{array}$ \\
\hline Tephrocactus muellerianus Backeb. & Lokrum & 1970 & 1990 & syn. Opuntia muelleriana \\
\hline Tephrocactus turpinii Lem. & Vienna & $\begin{array}{l}1964 \& 1968 \\
\& 1974\end{array}$ & $\begin{array}{l}1989 \text { \& no data } \\
\& 1982\end{array}$ & syn. Opuntia turpinii \\
\hline \multirow[t]{9}{*}{ Thelocactus setispinus (Engelm.) E.F. Anderson } & $\mathrm{nn}$ & 1949 & no data & 4 synonyms \\
\hline & nn & 1960 & 1972 & \\
\hline & Gent & 1961 & no data & \\
\hline & Vienna & 1960 & 1963 & \\
\hline & nn & $\begin{array}{l}\text { in collection } \\
\text { in } 1968\end{array}$ & 1975 & \\
\hline & Vienna & 1961 & 1968 & $\begin{array}{l}\text { arrived as Hamatocactus } \\
\text { setispinus }\end{array}$ \\
\hline & Erlangen & 1965 & 1968 & \\
\hline & Halle & 1964 & 1989 & \\
\hline & Goettingen & 1985 & 1990 & \\
\hline Trichocereus bridgesii (Salm-Dyck) Britton \& Rose & Barcelona & 1968 & 1989 & syn. Echinopsis lageniformis \\
\hline \multirow[t]{5}{*}{ Trichocereus macrogonus (Salm-Dyck) Riccob. } & nn & unknown & 1961 & $\begin{array}{l}\text { arrived as Cereus } \\
\text { macrogonus; one of } 5+ \\
\text { synonyms }\end{array}$ \\
\hline & nn & $\begin{array}{l}\text { in collection } \\
\text { in } 1963\end{array}$ & 1972 & \\
\hline & Liege & 1973 & 1990 & $\begin{array}{l}\text { arrived as Echinopsis } \\
\text { peruviana }\end{array}$ \\
\hline & Barcelona & 1980 & 2000 & \\
\hline & Barcelona & $\begin{array}{l}1967 \& 1971 \\
\& 1980\end{array}$ & $\begin{array}{l}1989 \& 1989 \& \\
2000\end{array}$ & arrived as T. pachanoi \\
\hline Trichocereus spachianus (Lem.) Riccob. & Liege & 1973 & 1989 & 2 synonyms \\
\hline Trichocereus strigosus (Salm-Dyck) Britton \& Rose & Berlin & 1953 & 1989 & 2 synonyms \\
\hline \multirow[t]{2}{*}{ Trichocereus thelegonoides (Speg.) Britton \& Rose } & Berlin & $\begin{array}{l}1961 \& 1963 \\
\& 1967 \& \\
2000\end{array}$ & $\begin{array}{l}1989 \& 2000 \& \\
2000 \& 2003\end{array}$ & 4 synonyms \\
\hline & Berlin & $\begin{array}{l}\text { in collection } \\
\text { in } 1968\end{array}$ & 1978 & \\
\hline Trichocereus vollianus Backeb. & Barcelona & 1980 & 1993 & syn. Echinopsis volliana \\
\hline $\begin{array}{l}\text { Turbinicarpus schmiedickeanus (Boed.) Buxb. \& } \\
\text { Backeb. subsp. schwarzii (Shurly) N.P. Taylor }\end{array}$ & Halle & 1979 & 1981 & arrived as T. schwarzii \\
\hline Weberbauerocereus johnsonii F.Ritter & Beograd & 1965 & 1978 & $20+$ synonyms \\
\hline Weberbauerocereus winterianus F. Ritter & Liege & 1973 & 1990 & 2 synonyms \\
\hline $\begin{array}{l}\text { Weberocereus tunilla (F.A.C. Weber) Britton \& } \\
\text { Rose subsp. biolleyi (F.A.C. Weber) Ralf Bauer }\end{array}$ & Gent & $1968 \& 1970$ & $1980 \& 1980$ & $\begin{array}{l}\text { arrived as W. panamensis; } \\
\text { one of } 2 \text { synonyms }\end{array}$ \\
\hline \multirow[t]{2}{*}{ Weingartia lanata F. Ritter } & nn & unknown & 1974 & $\begin{array}{l}\text { arrived as W. platygona; } \\
\text { one of } 5 \text { synonyms }\end{array}$ \\
\hline & Linz & 1972 & 1977 & \\
\hline \multirow[t]{4}{*}{ Weingartia neocumingii Backeb. } & Linz & 1972 & 1980 & $\begin{array}{l}\text { arrived as } W \text {. hediniana; } \\
\text { one of } 20+\text { synonyms }\end{array}$ \\
\hline & Linz & $1972 \& 2003$ & $1983 \& 2010$ & arrived as W. neocumingii \\
\hline & Chemnitz & 2007 & 2010 & \\
\hline & Chemnitz & 1997 & 2003 & $\begin{array}{l}\text { arrived as } W . \text { neocumingii } \\
\text { var. corroana }\end{array}$ \\
\hline \multicolumn{5}{|l|}{ Crassulaceae } \\
\hline \multirow[t]{2}{*}{ Adromischus cooperi (Baker) A.Berger } & Uppsala & 1958 & 1977 & 7 synonyms \\
\hline & Berlin & $1996 \& 1998$ & $2000 \& 2000$ & \\
\hline
\end{tabular}




\begin{tabular}{|c|c|c|c|c|}
\hline Scientific name & $\begin{array}{l}\text { City of plant } \\
\text { material origin }\end{array}$ & $\begin{array}{l}\text { Year of } \\
\text { ordering/ } \\
\text { purchasing }\end{array}$ & $\begin{array}{l}\text { Year when plant } \\
\text { was last recorded } \\
\text { in collection } \\
\end{array}$ & Notes and remarks \\
\hline $\begin{array}{l}\text { Adromischus cristatus (Haw.) Lem. var. clavifolius } \\
\text { (Haw.) Toelken }\end{array}$ & Wageningen & 1967 & 1970 & $\begin{array}{l}\text { arrived as A. poellnitzianus; } \\
\text { one of } 5 \text { synonyms }\end{array}$ \\
\hline Adromischus roaneanus Uitewaal & Wageningen & 1967 & 1968 & 2 synonyms \\
\hline Adromischus rotundifolius (Haw.) C.A.Sm. & Wageningen & $1963 \& 1965$ & $1972 \& 1979$ & $\begin{array}{l}\text { unresolved name (The } \\
\text { Plant List) }\end{array}$ \\
\hline \multirow[t]{5}{*}{ Aeonium arboreum Webb \& Berthel. } & Lisbon & 1970 & 1974 & 9 synonyms \\
\hline & Barcelona & 1971 & 1974 & \\
\hline & Bristol & 1977 & 1982 & \\
\hline & Wuppertal & 1986 & 2000 & \\
\hline & Marburg & 1986 & 2016 & \\
\hline \multirow[t]{3}{*}{$\begin{array}{l}\text { Aeonium arboreum var. atropurpureum (W.A. } \\
\text { Nicholson) A.Berger }\end{array}$} & Beograd & 1965 & 1965 & 3 synonyms \\
\hline & Berlin & $\begin{array}{l}1965 \& 1968 \\
\& 1970\end{array}$ & $\begin{array}{l}1968 \& 1977 \& \\
1972\end{array}$ & \\
\hline & Wageningen & 1966 & 1968 & \\
\hline \multirow[t]{3}{*}{ Aeonium arboreum var. holochrysum H.Y.Liu } & Amsterdam & 1971 & 1977 & $\begin{array}{l}\text { arrived as } A \text {. holochrysum; } \\
\text { one of } 4 \text { synonyms }\end{array}$ \\
\hline & Tenerife & 1984 & 1993 & \\
\hline & Bochum & 1987 & 1993 & \\
\hline \multirow[t]{4}{*}{ Aeonium aureum (C.Sm. ex Hornem.) T.H.M.Mes } & Brno & 1963 & 1966 & $\begin{array}{l}\text { arrived as Greenovia aurea, } \\
\text { one of } 5 \text { synonyms }\end{array}$ \\
\hline & Braunschweig & 1979 & 1979 & \\
\hline & Basel & $1986 \& 1988$ & $1990 \& 2000$ & \\
\hline & Duesseldorf & $1995 \& 2005$ & $2010 \& 2010$ & \\
\hline \multirow[t]{2}{*}{ Aeonium balsamiferum Webb \& Berthel. } & nn & 1952 & 1961 & 2 synonyms \\
\hline & Bristol & 1977 & 1990 & \\
\hline \multirow[t]{3}{*}{ Aeonium burchardii (Praeger) Praeger } & Berlin & $1965 \& 1970$ & $1972 \& 1978$ & $\begin{array}{l}\text { syn. Sempervivum } \\
\text { burchardii }\end{array}$ \\
\hline & nn & unknown & 1968 & \\
\hline & Muenchen & 1970 & 1983 & \\
\hline \multirow[t]{7}{*}{ Aeonium canariense (L.) Webb \& Berthel. } & Berlin & $\begin{array}{l}1962 \& 1965 \\
\& 1968 \& \\
1970\end{array}$ & $\begin{array}{l}1968 \& 1972 \& \\
1978 \& 1972\end{array}$ & $\begin{array}{l}\text { syn. Sempervivum } \\
\text { canariensa }\end{array}$ \\
\hline & Lisbon & 1970 & 1978 & \\
\hline & Adelaide & 1971 & 1972 & \\
\hline & Barcelona & 1971 & 1978 & \\
\hline & nn & 1976 & 1983 & \\
\hline & Basel & $1981 \& 1988$ & 1983 \& no data & \\
\hline & Marburg & 1986 & 1990 & syn. Sempervivum ciliatum \\
\hline $\begin{array}{l}\text { Aeonium canariense var. subplanum (Praeger) } \\
\text { H.Y.Liu }\end{array}$ & Basel & 1995 & 2000 & $\begin{array}{l}\text { arrived as A. subplanum; } \\
\text { one of } 3 \text { synonyms }\end{array}$ \\
\hline \multirow[t]{3}{*}{ Aeonium castello-paivae Bolle } & Berlin & $1978 \& 1979$ & $1978 \& 1990$ & syn. A. gomeraeum \\
\hline & $\begin{array}{l}\text { Marnier } \\
\text { (France) }\end{array}$ & 1977 & 1988 & \\
\hline & Bristol & 1977 & 1988 & \\
\hline \multirow[t]{3}{*}{ Aeonium ciliatum (Willd.) Webb \& Berthel. } & Bristol & 1977 & no data & \\
\hline & Basel & 1989 & 1993 & \\
\hline & Caen & 1993 & 1996 & \\
\hline \multirow[t]{3}{*}{ Aeonium decorum Webb ex Bolle } & nn & $\begin{array}{l}\text { in collection } \\
\text { in } 1961\end{array}$ & 1983 & \\
\hline & Tenerife & 1981 & 2000 & \\
\hline & Kiev & 1985 & 1988 & \\
\hline \multirow[t]{2}{*}{ Aeonium diplocyclum (Webb ex Bolle) T.H.M.Mes } & Berlin & 1970 & 1977 & $\begin{array}{l}\text { arrived as Greenovia } \\
\text { diplocycla }\end{array}$ \\
\hline & Saarbrucken & 1989 & 1993 & \\
\hline \multirow[t]{3}{*}{ Aeonium dodrantale (Willd.) T. Mes } & Berlin & $\begin{array}{l}1961 \& 1964 \\
\& 1979\end{array}$ & $\begin{array}{l}1968 \& 1971 \& \\
1988\end{array}$ & $\begin{array}{l}\text { arrived as Greenovia } \\
\text { dodrantalis; one of } 4 \\
\text { synonyms }\end{array}$ \\
\hline & Gent & 1963 & 1968 & \\
\hline & Basel & $1986 \& 1994$ & $1988 \& 2000$ & \\
\hline \multirow[t]{2}{*}{ Aeonium gomerense (Praeger) Praeger } & Amsterdam & 1955 & 1961 & $\begin{array}{l}\text { syn. Sempervivum } \\
\text { gomerense }\end{array}$ \\
\hline & Berlin & $1968 \& 1970$ & $1983 \& 1983$ & \\
\hline Aeonium gorgoneum J.A.Schmidt & Vienna & 1967 & 1978 & arrived as syn. $A$. webbii \\
\hline
\end{tabular}


S50 Sandev, D. et al.: Plethora of plants - collections of the Botanical Garden, Faculty of Science, University of Zagreb (2)

\begin{tabular}{|c|c|c|c|c|}
\hline Scientific name & $\begin{array}{l}\text { City of plant } \\
\text { material origin }\end{array}$ & $\begin{array}{l}\text { Year of } \\
\text { ordering/ } \\
\text { purchasing }\end{array}$ & $\begin{array}{l}\text { Year when plant } \\
\text { was last recorded } \\
\text { in collection }\end{array}$ & Notes and remarks \\
\hline \multirow[t]{2}{*}{ Aeonium haworthii Webb \& Berthel. } & nn & $\begin{array}{l}\text { in collection } \\
\text { in } 1961\end{array}$ & 1979 & $\begin{array}{l}\text { syn. Sempervivum } \\
\text { haworthii }\end{array}$ \\
\hline & Bristol & 1977 & 1983 & \\
\hline \multirow[t]{3}{*}{ Aeonium hierrense (R.P.Murray) Pit. \& Proust. } & Amsterdam & 1955 & no data & \\
\hline & Vienna & 1961 & 1961 & \\
\hline & $\begin{array}{l}\text { Marnier } \\
\text { (France) }\end{array}$ & 1977 & 1980 & \\
\hline Aeonium $x$ hybridum (Haw.) Rowley & $\mathrm{nn}$ & $\begin{array}{l}\text { in collection } \\
\text { in } 1961\end{array}$ & 2000 & $\begin{array}{l}\text { arrived as } A \text {. floribundum; } \\
\text { Aeonium simsii } \times A \text {. } \\
\text { spathulatum }\end{array}$ \\
\hline $\begin{array}{l}\text { Aeonium } \times \text { hybridum (Haw.) G.D.Rowley ' Blue } \\
\text { Moon' }\end{array}$ & Budapest & 1984 & 1985 & $\begin{array}{l}\text { arrived as Sempervivum } \\
\text { hybridum 'Blue Moon'; } \\
\text { hybrid between } \text { A. simsii × } \\
\text { A. spathulatum }\end{array}$ \\
\hline \multirow[t]{4}{*}{ Aeonium lindleyi Webb \& Berthel. } & Utrecht & 1958 & 1972 & syn. Sempervivum lindleyi \\
\hline & nn & $\begin{array}{l}\text { in collection } \\
\text { in } 1968\end{array}$ & 1982 & \\
\hline & Bologna & 1971 & 1990 & \\
\hline & Bristol & 1972 & 2000 & \\
\hline \multirow[t]{2}{*}{ Aeonium nobile (Praeger) Praeger } & Kiel & 1964 & 1968 & 2 synonyms \\
\hline & Marburg & 1989 & 1993 & \\
\hline \multirow[t]{2}{*}{ Aeonium percarneum (R.P.Murray) Pit. \& Proust. } & Berlin & $1967 \& 1978$ & $1977 \& 1980$ & 4 synonyms \\
\hline & nn & $\begin{array}{l}\text { in collection } \\
\text { in } 1970\end{array}$ & 1970 & \\
\hline \multirow[t]{3}{*}{ Aeonium sedifolium (Webb ex Bolle) Pit. \& Proust. } & Berlin & $1968 \& 1971$ & $1972 \& 1972$ & 2 synonyms \\
\hline & Wageningen & $1969 \& 1980$ & $1972 \& 1983$ & \\
\hline & Duisburg & 1989 & 1990 & \\
\hline \multirow[t]{2}{*}{ Aeonium simsii (Sweet) Stearn } & Berlin & $1967 \& 1968$ & $1972 \& 1974$ & syn. Sempervivum simsii \\
\hline & Gran Canaria & 1994 & 2000 & \\
\hline \multirow[t]{4}{*}{ Aeonium spathulatum (Hornem.) Praeger } & Berlin & $1965 \& 1976$ & $1966 \& 1982$ & 5 synonyms \\
\hline & Besancon & 1965 & 1970 & \\
\hline & Braunschweig & 1980 & 1983 & \\
\hline & Gran Canaria & 1994 & 2000 & \\
\hline \multirow[t]{4}{*}{ Aeonium tabuliforme (Haw.) Webb \& Berthel. } & Wuppertal & 1998 & 2000 & 5 synonyms \\
\hline & Bochum & 1998 & 2000 & \\
\hline & Kiel & 1997 & 2000 & \\
\hline & Innsbruck & 1999 & 2003 & \\
\hline \multirow[t]{4}{*}{$\begin{array}{l}\text { Aeonium urbicum (C. Sm. ex Hornem.) Webb \& } \\
\text { Berthel. }\end{array}$} & Marburg & 1953 & 1961 & syn. Sempervivum urbicum \\
\hline & Madrid & 1980 & 1983 & \\
\hline & Duisburg & 1986 & 1990 & \\
\hline & Gran Canaria & 1994 & 2000 & \\
\hline Aeonium valverdense (Praeger) Praeger & Gran Canaria & 1994 & 2000 & \\
\hline Aeonium viscatum D.A. Webb & Bristol & 1977 & 2000 & $\begin{array}{l}\text { syn. A. lindleyi var. } \\
\text { viscatum }\end{array}$ \\
\hline \multirow[t]{2}{*}{ Aichryson $x$ aizoides (Lamarck) E. C. Nelson } & nn & $\begin{array}{l}\text { in collection } \\
\text { in } 1963\end{array}$ & 1966 & $\begin{array}{l}\text { arrived as Aeonium } \\
\text { domesticum; most probably } \\
\text { Aichryson punctatum (or } \\
\text { divaricatum) } \mathrm{A} \text { A. tortuosum }\end{array}$ \\
\hline & Kijev & 1986 & 1990 & \\
\hline \multirow[t]{3}{*}{ Aichryson laxum (Haw.) Bramwell } & Berlin & 1968 & 1972 & $\begin{array}{l}\text { arrived as A. dichotomum; } \\
\text { one of } 5+\text { synonyms }\end{array}$ \\
\hline & Basel & 1971 & 1972 & \\
\hline & Coimbra & 1962 & 1963 & \\
\hline \multirow[t]{3}{*}{$\begin{array}{l}\text { Aichryson punctatum (C. Sm. ex Link) Webb \& } \\
\text { Berthel. }\end{array}$} & Muenchen & 1970 & 1972 & 5 synonyms \\
\hline & Tenerife & 1987 & 2000 & \\
\hline & Tuebingen & 1988 & 2000 & \\
\hline \multirow[t]{3}{*}{ Aichryson tortuosum (Aiton) Webb \& Berthel. } & nn & $\begin{array}{l}\text { in collection } \\
\text { in } 1966\end{array}$ & 1972 & 2 synonyms \\
\hline & Kijev & 1985 & 1990 & \\
\hline & Duisburg & 1989 & 2004 & \\
\hline Altamiranoa elongata Rose & Wageningen & 1955 & 1961 & from cuttings; 2 synonyms \\
\hline
\end{tabular}




\begin{tabular}{|c|c|c|c|c|}
\hline Scientific name & $\begin{array}{l}\text { City of plant } \\
\text { material origin }\end{array}$ & $\begin{array}{l}\text { Year of } \\
\text { ordering/ } \\
\text { purchasing }\end{array}$ & $\begin{array}{l}\text { Year when plant } \\
\text { was last recorded } \\
\text { in collection }\end{array}$ & Notes and remarks \\
\hline Bryophyllum delagoense (Eckl. \& Zeyh.) Druce & nn & 1949 & 1966 & $\begin{array}{l}\text { arrived as B. tubiflorum; } \\
\text { one of } 7 \text { synonyms; from } \\
\text { cuttings }\end{array}$ \\
\hline $\begin{array}{l}\text { Bryophyllum fedtschenkoi (Raym.-Hamet \& } \\
\text { H.Perrier) Lauz.-March. }\end{array}$ & Genova & 1967 & 1981 & $\begin{array}{l}\text { arrived as Kalanchoe } \\
\text { fedtschenkoi; one of } 2 \\
\text { synonyms }\end{array}$ \\
\hline Bryophyllum laxiflorum (Baker) Govaerts & $\mathrm{nn}$ & 1952 & 1961 & $\begin{array}{l}\text { arrived as B. crenatum; } \\
\text { from cuttings }\end{array}$ \\
\hline Bryophyllum pinnatum (Lam.) Oken & nn & 1952 & 1963 & $\begin{array}{l}\text { arrived as B. calycium; } \\
\text { from cuttings }\end{array}$ \\
\hline \multirow[t]{2}{*}{ Chiastophyllum oppositifolium (Ledeb.) A.Berger } & Lausanne & 1962 & 1963 & $\begin{array}{l}\text { arrived as Cotyledon } \\
\text { oppositifolius; one of } 2 \\
\text { synonyms }\end{array}$ \\
\hline & Berlin & 1973 & 1974 & \\
\hline \multirow[t]{3}{*}{ Cotyledon orbiculata L. } & Rotterdam & 1960 & 1961 & \\
\hline & Barcelona & 1971 & 1978 & \\
\hline & Muenchen & 1976 & 1982 & \\
\hline Cotyledon orbiculata var. oblonga (Haw.) DC. & Harrogate & 1991 & 2000 & $\begin{array}{l}\text { arrived as C. macrantha; } \\
\text { one of } 20+\text { synonyms }\end{array}$ \\
\hline Crassula alba Forssk. & Muenchen & $1966 \& 1970$ & $1968 \& 1971$ & \\
\hline \multirow[t]{2}{*}{ Crassula arborescens (Mill.) Willd. } & $\mathrm{nn}$ & 1952 & 1961 & $\begin{array}{l}\text { syn. C. arborescens subsp. } \\
\text { arborescens; from cuttings }\end{array}$ \\
\hline & Besancon & 1971 & 1990 & \\
\hline \multirow[t]{2}{*}{$\begin{array}{l}\text { Crassula atropurpurea (Haw.) D.Dietr. } \\
\text { var. watermeyeri (Compton) Toelken }\end{array}$} & Wageningen & $1962 \& 1963$ & $2000 \& 1980$ & $\begin{array}{l}\text { arrived as C. sericea auct.; } \\
\text { one of } 3 \text { synonyms }\end{array}$ \\
\hline & nn & $\begin{array}{l}\text { in collection } \\
\text { in } 1968\end{array}$ & 1972 & \\
\hline Crassula barbata Thunb. & Kirstenbosch & 1954 & 1961 & \\
\hline \multirow[t]{2}{*}{ Crassula brevifolia Harv. } & Wageningen & 1967 & 1978 & from cuttings \\
\hline & Berlin & 1989 & 1993 & \\
\hline $\begin{array}{l}\text { Crassula capitella Thunb. subsp. nodulosa (Schönl.) } \\
\text { Toelken }\end{array}$ & Berlin & 1968 & 1972 & arrived as C. nodulosa \\
\hline \multirow[t]{3}{*}{$\begin{array}{l}\text { Crassula capitella subsp. thyrsiflora (Thunb.) } \\
\text { Toelken }\end{array}$} & Wageningen & $\begin{array}{l}\text { unknown \& } \\
1972\end{array}$ & $1961 \& 1972$ & arrived as C. corymbulosa \\
\hline & Berlin & 1979 & 1982 & arrived as $\mathrm{C}$. triebneri \\
\hline & Gent & 1979 & 1982 & \\
\hline Crassula ciliata $\mathrm{L}$. & Kirstenbosch & 1954 & no data & \\
\hline \multirow[t]{2}{*}{ Crassula coccinea $\mathrm{L}$. } & Kirstenbosch & 1976 & 1980 & $\begin{array}{l}\text { arrived as Rochea coccinea; } \\
\text { one of } 10+\text { synonyms }\end{array}$ \\
\hline & Innsbruck & 2000 & 2003 & \\
\hline Crassula corallina L.f. subsp. macrorrhiza Toelken & Dresden & 2002 & 2003 & arrived as C. corallina \\
\hline Crassula cordata Thunb. & Wageningen & 1955 & 1961 & from cuttings \\
\hline Crassula cotyledonis Thunb. & Wageningen & $\begin{array}{l}1955 \& \text { in } \\
\text { collection in } \\
1965\end{array}$ & $1961 \& 1978$ & $\begin{array}{l}\text { arrived as C. dubia; from } \\
\text { cuttings (1955) }\end{array}$ \\
\hline Crassula crenulata Thunb. & Berlin & 1968 & 1972 & \\
\hline Crassula cultrata $\mathrm{L}$. & Wageningen & 1955 & 1963 & $\begin{array}{l}\text { from cuttings; syn. } \\
\text { Globulea cultrata }\end{array}$ \\
\hline Crassula dejecta Jacq. & Wageningen & $1955 \& 1967$ & $1961 \& 2003$ & $\begin{array}{l}\text { 20+ synonyms; from } \\
\text { cuttings (1967) }\end{array}$ \\
\hline Crassula elegans Schönland \& Baker f. & Berlin & 1979 & 1982 & $\begin{array}{l}\text { arrived as C. humilis; syn. } \\
\text { Crassula elegans ssp. } \\
\text { elegans }\end{array}$ \\
\hline Crassula ericoides Haw. & $\mathrm{nn}$ & 1949 & 1979 & \\
\hline \multirow[t]{2}{*}{$\begin{array}{l}\text { Crassula exilis Harv. subsp. schmidtii (Regel) } \\
\text { G.D.Rowley }\end{array}$} & nn & 1951 & no data & $\begin{array}{l}\text { arrived as C. schmidtii; } \\
\text { from cuttings }\end{array}$ \\
\hline & Berlin & 1973 & 1982 & \\
\hline \multirow{4}{*}{ Crassula exilis subsp. sedifolia (N.E.Br.) Toelken } & Gent & 1963 & 1978 & arrived as C. picturata \\
\hline & Berlin & 1968 & 1990 & \\
\hline & nn & $\begin{array}{l}\text { in collection } \\
\text { in } 1975\end{array}$ & 1988 & \\
\hline & nn & 1952 & 1961 & arrived as C. cooperi \\
\hline
\end{tabular}


S52 Sandev, D. et al.: Plethora of plants - collections of the Botanical Garden, Faculty of Science, University of Zagreb (2)

\begin{tabular}{|c|c|c|c|c|}
\hline Scientific name & $\begin{array}{l}\text { City of plant } \\
\text { material origin }\end{array}$ & $\begin{array}{l}\text { Year of } \\
\text { ordering/ } \\
\text { purchasing }\end{array}$ & $\begin{array}{l}\text { Year when plant } \\
\text { was last recorded } \\
\text { in collection }\end{array}$ & Notes and remarks \\
\hline \multirow[t]{3}{*}{ Crassula fascicularis Lam. } & nn & 1952 & 1961 & $\begin{array}{l}\text { arrived as Rochea } \\
\text { odoratissima; one of } 10+ \\
\text { synonyms }\end{array}$ \\
\hline & Graz & 1970 & 1971 & \\
\hline & Berlin & 1968 & 1971 & arrived as Rochea versicolor \\
\hline \multirow[t]{2}{*}{ Crassula 'Justus Corderoy' } & Uppsala & 1958 & 1980 & $\begin{array}{l}\text { known cultivar (Crassula } \\
\text { exilis ssp. cooperi } \mathrm{x} C \text {. } \\
\text { perfoliata var. minor) }\end{array}$ \\
\hline & Kijev & 1985 & 1990 & \\
\hline Crassula lactea Aiton & nn & $\begin{array}{l}\text { in collection } \\
\text { in } 1963\end{array}$ & 1970 & \\
\hline \multirow[t]{3}{*}{$\begin{array}{l}\text { Crassula lanceolata (Eckl. \& Zeyh.) Endl. ex Walp. } \\
\text { subsp. lanceolata }\end{array}$} & nn & $\begin{array}{l}\text { in collection } \\
\text { in } 1961\end{array}$ & 2000 & $\begin{array}{l}\text { arrived as } C \text {. lycopodioides; } \\
5 \text { synonyms }\end{array}$ \\
\hline & Besancon & 1971 & 1971 & \\
\hline & Berlin & 1998 & 2000 & \\
\hline Crassula lanuginosa Harv. & Berlin & 1968 & 2000 & 2 synonyms \\
\hline \multirow[t]{2}{*}{$\begin{array}{l}\text { Crassula lanuginosa var. pachystemon (Schönl. \& } \\
\text { Baker f.) Toelken }\end{array}$} & Berlin & 1970 & 1978 & arrived as C. ernestii \\
\hline & Giessen & 1989 & 1990 & \\
\hline Crassula lycopodioides Lam. var. variegata E. Lamb & Wageningen & 1971 & 1980 & \\
\hline \multirow[t]{2}{*}{$\begin{array}{l}\text { Crassula lycopodioides var. pseudolycopodioides } \\
\text { (Dinter) Walther ex Jacobsen }\end{array}$} & nn & 1955 & 1961 & $\begin{array}{l}\text { arrived as C. pseudolycopo- } \\
\text { dioides; from cuttings }\end{array}$ \\
\hline & Wageningen & $1971 \& 1972$ & $1972 \& 1972$ & $\begin{array}{l}\text { arrived as C. lycopodioides } \\
\text { var. pseudolycopodioides }\end{array}$ \\
\hline $\begin{array}{l}\text { Crassula multicava Lem. subsp. floribunda } \\
\text { Friedrich ex Toelken }\end{array}$ & Rome & 1955 & 1963 & $\begin{array}{l}\text { arrived as C. multicava; } \\
\text { from cuttings }\end{array}$ \\
\hline Crassula multiflora Schönland \& Baker f. & Berlin & $1968 \& 1979$ & $1983 \& 1983$ & $\begin{array}{l}\text { syn. C. multiflora } \\
\text { subsp. multiflora }\end{array}$ \\
\hline Crassula muricata Thunb. & nn & 1955 & no data & $\begin{array}{l}\text { arrived as C. divaricata; } \\
\text { from cuttings }\end{array}$ \\
\hline \multirow[t]{7}{*}{ Crassula muscosa L. var. muscosa } & nn & $\begin{array}{l}\text { in collection } \\
\text { in } 1963\end{array}$ & 1968 & $\begin{array}{l}\text { arrived as C. lycopodioides } \\
\text { var. pseudolycopodioides; } \\
\text { one of } 10+\text { synonyms }\end{array}$ \\
\hline & nn & unknown & 1968 & $\begin{array}{l}\text { arrived as C. lycopodioides } \\
\text { var. monstrosa hort. }\end{array}$ \\
\hline & Szeged & 1999 & 2000 & \\
\hline & nn & 1955 & 1961 & $\begin{array}{l}\text { arrived as C. pseudolycopo- } \\
\text { dioides; from cuttings }\end{array}$ \\
\hline & Besancon & 1971 & 1972 & $\begin{array}{l}\text { arrived as C. lycopodioides } \\
\text { var. purpusii }\end{array}$ \\
\hline & Wageningen & 1974 & 1981 & \\
\hline & Berlin & 2001 & 2016 & from cuttings \\
\hline \multirow[t]{4}{*}{ Crassula nudicaulis L. } & Wageningen & $1955 \& 1963$ & $1963 \& 1972$ & $\begin{array}{l}\text { arrived as C. cephalophora; } \\
\text { from cuttings (1955) }\end{array}$ \\
\hline & Berlin & 1955 & 1980 & arrived as C. obvallata \\
\hline & nn & $\begin{array}{l}\text { in collection } \\
\text { in } 1963\end{array}$ & 1968 & \\
\hline & Muenchen & 1972 & 1972 & \\
\hline \multirow[t]{2}{*}{ Crassula obovata Haw. } & Wageningen & 1955 & 1961 & $\begin{array}{l}\text { arrived as } C \text {. reversisetos; } \\
\text { from cuttings }\end{array}$ \\
\hline & Muenchen & 1966 & 1968 & $\begin{array}{l}\text { arrived as C. ramuliflora; } \\
\text { from cuttings; syn. Sedum } \\
\text { ramuliflorum }\end{array}$ \\
\hline Crassula orbicularis L. & Wageningen & $\begin{array}{l}1955 \& 1964 \\
\& 1965\end{array}$ & $\begin{array}{l}1961 \& 1968 \& \\
1988\end{array}$ & $\begin{array}{l}\text { syn. C. rosularis; from } \\
\text { cuttings }\end{array}$ \\
\hline \multirow[t]{5}{*}{ Crassula ovata (Mill.) Druce } & nn & $\begin{array}{l}\text { in collection } \\
\text { in } 1972\end{array}$ & 2000 & 8 synonyms \\
\hline & Vienna & 1958 & 1961 & arrived as C. portulacea \\
\hline & Roma & 1960 & 1966 & \\
\hline & Roma & 1960 & 1961 & arrived as C. obliqua \\
\hline & nn & $\begin{array}{l}\text { in collection } \\
\text { in } 1963\end{array}$ & 1972 & \\
\hline \multirow[t]{2}{*}{$\begin{array}{l}\text { Crassula pellucida L. subsp. marginalis (Sol. ex } \\
\text { Aiton) Toelken }\end{array}$} & nn & unknown & 1961 & arrived as C. marginalis \\
\hline & $\mathrm{nn}$ & unknown & 1963 & \\
\hline
\end{tabular}




\begin{tabular}{|c|c|c|c|c|}
\hline Scientific name & $\begin{array}{l}\text { City of plant } \\
\text { material origin }\end{array}$ & $\begin{array}{l}\text { Year of } \\
\text { ordering/ } \\
\text { purchasing }\end{array}$ & $\begin{array}{l}\text { Year when plant } \\
\text { was last recorded } \\
\text { in collection }\end{array}$ & Notes and remarks \\
\hline & Wageningen & 1971 & 1977 & \\
\hline \multirow[t]{4}{*}{$\begin{array}{l}\text { Crassula perfoliata L. var. falcata (J.C.Wendl.) } \\
\text { Toelken }\end{array}$} & $\begin{array}{lll}0 & 0 \\
\end{array}$ & $\begin{array}{l}\text { in collection } \\
\text { in } 1963\end{array}$ & 1975 & arrived as C. falcata \\
\hline & Besancon & 1971 & 1972 & \\
\hline & Ferrara & 1970 & 1972 & \\
\hline & Cluj-Napoca & 1972 & 1972 & \\
\hline \multirow[t]{2}{*}{$\begin{array}{l}\text { Crassula perfoliata var. coccinea (Sweet) G.D. } \\
\text { Rowley }\end{array}$} & nn & 1952 & 1961 & arrived as C. perfoliata \\
\hline & Muenchen & 1977 & 1978 & \\
\hline \multirow[t]{4}{*}{ Crassula perforata Thunb. } & Wageningen & $\begin{array}{l}1959 \& 1962 \\
\& 1963 \& \\
1964 \& 1971\end{array}$ & $\begin{array}{l}1978 \& 1990 \& \text { \& } \\
1990 \& 1968 \& \\
1990\end{array}$ & $\begin{array}{l}\text { arrived as C. nealeana; } 10 \\
\text { synonyms }\end{array}$ \\
\hline & Wageningen & 1965 & 1970 & arrived as C. conjuncta \\
\hline & Muenchen & 1977 & 1978 & \\
\hline & Wageningen & 1959 & 1968 & arrived as C. perfossa Lam. \\
\hline Crassula perforata Thunb. 'Giant Pagoda' & Wageningen & $1969 \& 1971$ & $1972 \& 1983$ & unknown cultivar \\
\hline $\begin{array}{l}\text { Crassula pubescens Thunb. subsp. radicans (Haw.) } \\
\text { Toelken }\end{array}$ & Wageningen & 1955 & 1961 & arrived as $C$. radicans \\
\hline \multirow[t]{2}{*}{$\begin{array}{l}\text { Crassula rupestris L. f. subsp. marnierana (H. E. } \\
\text { Huber \& Jacobsen) Toelken }\end{array}$} & nn & unknown & 1963 & arrived as C. marnieriana \\
\hline & Lokrum & 1970 & 1972 & \\
\hline Crassula sarcocaulis Eckl. \& Zeyh. & Wageningen & 1955 & 1972 & $\begin{array}{l}\text { syn. C. sarcocaulis } \\
\text { subsp. rupicola; from } \\
\text { cuttings }\end{array}$ \\
\hline \multirow[t]{2}{*}{ Crassula sarmentosa Harv. } & nn & unknown & 1963 & 2 synonyms \\
\hline & Duisburg & 1990 & 2000 & \\
\hline \multirow[t]{3}{*}{$\begin{array}{l}\text { Crassula schimperi Fisch. \& C.A.Mey. subsp. } \\
\text { phyturus (Mildbr.) R.Fern. }\end{array}$} & Wageningen & 1955 & 1961 & arrived as C. phyturus \\
\hline & $\mathrm{nn}$ & $\begin{array}{l}\text { in collection } \\
\text { in } 1963\end{array}$ & 1980 & \\
\hline & Duisburg & 1989 & 1990 & \\
\hline $\begin{array}{l}\text { Crassula setulosa Harv. var. rubra (N.E. Br.) G.D. } \\
\text { Rowley }\end{array}$ & Berlin & 1979 & 1989 & $\begin{array}{l}\text { arrived as C. curta; syn. C. } \\
\text { curta var. rubra }\end{array}$ \\
\hline \multirow[t]{2}{*}{ Crassula socialis Schönland } & $\mathrm{nn}$ & 1952 & 1978 & \\
\hline & Wageningen & $\begin{array}{l}1970 \& 1974 \\
\& 1980\end{array}$ & $\begin{array}{l}1978 \& 1978 \& \\
1982\end{array}$ & \\
\hline \multirow[t]{2}{*}{ Crassula spathulata Thunb. } & Wageningen & 1956 & 1963 & 4 synonyms \\
\hline & Szeged & 1970 & 2000 & \\
\hline Crassula subaphylla (Eckl. \& Zeyh.) Harv. & Wageningen & 1958 & 1963 & \\
\hline Crassula subaphylla var. subaphylla & Goeteborg & 1978 & 1980 & $\begin{array}{l}\text { arrived as C. remota; one } \\
\text { of } 2 \text { synonyms }\end{array}$ \\
\hline Crassula tabularis Dinter & Berlin & 1973 & 1978 & \\
\hline Crassula tecta Thunb. & Muenchen & 1977 & 1979 & syn. Purgosea tecta \\
\hline \multirow[t]{3}{*}{ Crassula tetragona $\mathrm{L}$. } & nn & \begin{tabular}{|l|l|}
$1956 \&$ \& \\
unknown
\end{tabular} & $1961 \& 1963$ & from cuttings (1956) \\
\hline & Szeged & 1970 & 1980 & \\
\hline & Wageningen & $1971 \& 1980$ & $1988 \& 1981$ & \\
\hline \multirow[t]{2}{*}{ Crassula 'Tom Thumb' } & Wageningen & $\begin{array}{l}1962 \& 1963 \\
\& 1974\end{array}$ & $\begin{array}{l}1980 \& 1968 \& \\
1978\end{array}$ & $\begin{array}{l}\text { hybrid between } C \text {. } \\
\text { rupestris } \times \text { C. rupestris } \\
\text { subsp. Marnieriana }\end{array}$ \\
\hline & Berlin & 1973 & 1975 & \\
\hline $\begin{array}{l}\text { Crassula tomentosa Thumb. var. glabrifolia (Harv.) } \\
\text { G.D. Rowley }\end{array}$ & Berlin & 1968 & 1972 & $\begin{array}{l}\text { arrived as C. interrupta; } \\
\text { syn. C. tomentosa var. inter- } \\
\text { rupta }\end{array}$ \\
\hline Dudleya candelabrum Rose & Graz & 1972 & 1988 & 2 synonyms \\
\hline \multirow[t]{2}{*}{ Dudleya cymosa (Lem.) Britton \& Rose } & nn & $\begin{array}{l}\text { in collection } \\
\text { in } 1963\end{array}$ & 1968 & $\begin{array}{l}\text { arrived as D. pursii; one of } \\
3 \text { synonyms }\end{array}$ \\
\hline & Cantonspark & 1953 & no data & \\
\hline Dudleya greenei Rose & Berlin & 1978 & 1983 & 2 synonyms \\
\hline Dudleya ingens Rose & Gent & 1964 & 1980 & $\begin{array}{l}\text { arrived as D. viridicata; } \\
\text { one of } 2 \text { synonyms }\end{array}$ \\
\hline Dudleya virens (Rose) Moran & $\begin{array}{l}\text { San Marino } \\
\text { (California) }\end{array}$ & 1984 & 1990 & 2 synonyms \\
\hline Echeveria affinis E.Walther & Wageningen & 1969 & 1972 & \\
\hline
\end{tabular}


S54 Sandev, D. et al.: Plethora of plants - collections of the Botanical Garden, Faculty of Science, University of Zagreb (2)

\begin{tabular}{|c|c|c|c|c|}
\hline Scientific name & $\begin{array}{l}\text { City of plant } \\
\text { material origin }\end{array}$ & $\begin{array}{l}\text { Year of } \\
\text { ordering/ } \\
\text { purchasing }\end{array}$ & $\begin{array}{l}\text { Year when plant } \\
\text { was last recorded } \\
\text { in collection }\end{array}$ & Notes and remarks \\
\hline \multirow[t]{3}{*}{ Echeveria agavoides Lem. } & nn & $\begin{array}{l}\text { in collection } \\
\text { in } 1961\end{array}$ & 1966 & 5 synonyms \\
\hline & Wageningen & 1967 & 1968 & \\
\hline & Barcelona & 1971 & 1975 & \\
\hline Echeveria agavoides Lem. 'Cristata' & $\mathrm{nn}$ & 1950 & 1963 & \\
\hline Echeveria albicans Walther & Wageningen & 1959 & 1966 & $\begin{array}{l}\text { arrived as E. elegans var. } \\
\text { kesselringiana }\end{array}$ \\
\hline \multirow{3}{*}{ Echeveria amoena De Smet ex E.Morren } & Wageningen & 1958 & 1981 & syn. E. pusilla \\
\hline & nn & $\begin{array}{l}\text { in collection } \\
\text { in } 1968\end{array}$ & 2000 & \\
\hline & Liege & 1984 & 2016 & \\
\hline \multirow[t]{2}{*}{ Echeveria coccinea (Cav.) DC. } & nn & $\begin{array}{l}\text { in collection } \\
\text { in } 1961\end{array}$ & 1972 & arrived as E. pubescens \\
\hline & Basel & 1995 & 2000 & syn. Cotyledon coccinea \\
\hline \multirow[t]{5}{*}{ Echeveria elegans Rose } & Berlin & 1955 & 1961 & 4 synonyms \\
\hline & Cantonspark & 1955 & 1966 & \\
\hline & Caen & 2002 & 2003 & \\
\hline & Berlin & 1968 & 1972 & arrived as E. harmsii \\
\hline & Caen & 2002 & 2016 & \\
\hline Echeveria fulgens Lem. & Berlin & 1981 & 1988 & 2 synonyms \\
\hline Echeveria $x$ gilva E. Walther & Wageningen & $\begin{array}{l}1965 \& 1967 \\
\& 1971\end{array}$ & $\begin{array}{l}1972 \& 1979 \& \\
1983\end{array}$ & E. agavoides $x$ E. elegans \\
\hline Echeveria grandifolia Haw. & Amsterdam & 1967 & 1972 & arrived as E. campanulata \\
\hline \multirow[t]{3}{*}{ Echeveria multicaulis Rose } & nn & $\begin{array}{l}1953 \& \text { in } \\
\text { collection in } \\
1963\end{array}$ & no data \& 2003 & from cuttings (1953) \\
\hline & Bern & 1953 & no data & \\
\hline & Giessen & 1985 & 1993 & \\
\hline Echeveria nodulosa (Baker) Otto & Debrecen & 1956 & 1961 & $\begin{array}{l}\text { arrived as Cotyledon } \\
\text { nodulosa }\end{array}$ \\
\hline \multirow[t]{2}{*}{ Echeveria puchella Berger } & Wageningen & $\begin{array}{l}1963 \& 1964 \\
\& 1965\end{array}$ & $\begin{array}{l}2000 \& 1966 \& \& \\
1966\end{array}$ & \\
\hline & $\mathrm{nn}$ & unknown & 1968 & \\
\hline Echeveria quitensis (Kunth) Lindl. & Bruxelles & $1961 \& 1964$ & $1962 \& 1966$ & syn. Sedum quitense \\
\hline \multirow[t]{3}{*}{ Echeveria rosea Lindl. } & Napulj & 1960 & 1961 & 3 synonyms \\
\hline & $\mathrm{nn}$ & unknown & $1963 \& 1974$ & \\
\hline & Berlin & 1968 & 1971 & \\
\hline Echeveria scaphophylla hort. & Uppsala & 1958 & 1963 & $\begin{array}{l}\text { Echeveria agavoides } \mathrm{x} \\
\text { Cremnophila linguifolia }\end{array}$ \\
\hline \multirow[t]{2}{*}{ Echeveria secunda Booth ex Lindl. } & nn & unknown & $\begin{array}{l}1961 \& 1963 \& \& \\
1970\end{array}$ & $\begin{array}{l}\text { arrived as E. glauca; one of } \\
2 \text { synonyms }\end{array}$ \\
\hline & Wageningen & 1980 & 1988 & \\
\hline \multirow[t]{2}{*}{ Echeveria secunda Booth ex Lindl. 'Cristata' } & Wageningen & $1963 \& 1965$ & $1980 \& 1988$ & arrived as E. glauca cristata \\
\hline & nn & unknown & 1962 & \\
\hline Echeveria sedoides E.Walther & Liege & 1984 & 1988 & syn. E. macdougallii \\
\hline Echeveria setosa Rose \& Purpus & nn & $\begin{array}{l}\text { in collection } \\
\text { in } 1961\end{array}$ & 2000 & \\
\hline \multirow[t]{2}{*}{ Graptopetalum macdougallii Alexander } & Wageningen & $\begin{array}{l}1963 \& 1967 \\
\& 1971 \& \\
1972 \& 1980\end{array}$ & $\begin{array}{l}1983 \& 1968 \& \\
1983 \& 1975 \& \\
1983\end{array}$ & from cuttings (1967) \\
\hline & Goeteborg & 1978 & 1979 & \\
\hline Graptopetalum pachyphyllum Rose & Wageningen & 1972 & 1978 & 2 synonyms \\
\hline \multirow[t]{5}{*}{ Graptopetalum paraguayense (N.E.Br.) E.Walther } & nn & $1954 \& 1955$ & no data & $\begin{array}{l}\text { arrived as Echeveria } \\
\text { weinbergii; one of } 5+ \\
\text { synonyms }\end{array}$ \\
\hline & Szeged & 1970 & 1980 & \\
\hline & Bologna & 1971 & 1971 & \\
\hline & Wageningen & $1955 \& 1964$ & $1961 \& 1968$ & $\begin{array}{l}\text { arrived as G. paraguayense; } \\
\text { from cuttings (1955) }\end{array}$ \\
\hline & nn & $\begin{array}{l}\text { in collection } \\
\text { in } 1968\end{array}$ & 1978 & \\
\hline
\end{tabular}




\begin{tabular}{|c|c|c|c|c|}
\hline Scientific name & $\begin{array}{l}\text { City of plant } \\
\text { material origin }\end{array}$ & $\begin{array}{l}\text { Year of } \\
\text { ordering/ } \\
\text { purchasing }\end{array}$ & $\begin{array}{l}\text { Year when plant } \\
\text { was last recorded } \\
\text { in collection }\end{array}$ & Notes and remarks \\
\hline$\times$ Graptoveria Rowley cv. acaulis & Wageningen & 1967 & 1968 & $\begin{array}{l}\text { from cuttings; (Graptopeta- } \\
\text { lum } x \text { Echeveria = hybrids } \\
\text { common in cultivation } \\
\text { and difficult to identify) }\end{array}$ \\
\hline \multirow[t]{3}{*}{ Hylotelephium erythrostictum (Miq.) H. Ohba } & Paris & 1970 & 1990 & $\begin{array}{l}\text { arrived as Sedum } \\
\text { alboroseum; one of } 4 \\
\text { synonyms }\end{array}$ \\
\hline & Vladivostok & 1971 & 1972 & \\
\hline & Budapest & 1975 & 1978 & \\
\hline $\begin{array}{l}\text { Hylotelephium spectabile (Boreau) H. Ohba } \\
\text { 'Roseolum' }\end{array}$ & Budapest & 1971 & 1971 & $\begin{array}{l}\text { arrived as Sedum spectabile } \\
\text { 'Roseolum' }\end{array}$ \\
\hline \multirow[t]{2}{*}{ Kalanchoe beauverdii Raym.-Hamet } & Antwerpen & $\begin{array}{l}1965 \& 1972 \\
\& 1975\end{array}$ & $\begin{array}{l}1966 \& 2000 \& \\
1983\end{array}$ & $10+$ synonyms \\
\hline & Nantes & 1996 & 2000 & \\
\hline \multirow[t]{3}{*}{ Kalanchoe blossfeldiana Poelln. } & nn & 1952 & 1961 & 2 synonyms \\
\hline & Nancy & 1971 & 1971 & \\
\hline & Košice & 1978 & 2000 & \\
\hline Kalanchoe blossfeldiana Poelln. 'Alfred Graser' & Nancy & 1971 & 1990 & $\begin{array}{l}\text { syn. K. globulifera var. } \\
\text { coccinea }\end{array}$ \\
\hline \multirow[t]{2}{*}{ Kalanchoe blossfeldiana Poelln. 'Brilliant Star' } & Nancy & 1971 & 1988 & $\begin{array}{l}\text { syn. K. globulifera var. } \\
\text { coccinea }\end{array}$ \\
\hline & Košice & 1978 & 1982 & \\
\hline \multirow[t]{2}{*}{ Kalanchoe blossfeldiana Poelln. 'Ernst Thiede' } & nn & 1951 & 1971 & $\begin{array}{l}\text { syn. K. globulifera var. } \\
\text { coccinea }\end{array}$ \\
\hline & Gent & $1970 \& 1974$ & $1988 \& 1978$ & \\
\hline \multirow[t]{2}{*}{ Kalanchoe blossfeldiana Poelln. 'Feuerblute' } & Gent & 1970 & 1974 & $\begin{array}{l}\text { syn. K. globulifera var. } \\
\text { coccinea }\end{array}$ \\
\hline & Nancy & 1971 & 1993 & \\
\hline \multirow[t]{2}{*}{ Kalanchoe campanulata (Baker) Baill. } & Antwerpen & $\begin{array}{l}1966 \& 1967 \\
\& 1970\end{array}$ & $\begin{array}{l}1977 \& \text { \& } 1971 \& \\
1970\end{array}$ & $\begin{array}{l}\text { syn. Kitchingia } \\
\text { campanulata; from cuttings } \\
\text { (1967) }\end{array}$ \\
\hline & Meise & 1986 & 1988 & \\
\hline \multirow[t]{3}{*}{ Kalanchoe citrina Schweinf. } & Geneve & 1978 & 1988 & syn. K. citrina var. erythreae \\
\hline & Muenchen & $1977 \& 1995$ & $1988 \& 2000$ & \\
\hline & Reading & 1997 & 2000 & \\
\hline Kalanchoe crenata (Andrews) Haw. & $\begin{array}{l}\text { Alberta-Ed- } \\
\text { monton }\end{array}$ & $1960 \& 1961$ & $1960 \& 1961$ & arrived as K. brasiliensis \\
\hline \multirow[t]{3}{*}{$\begin{array}{l}\text { Kalanchoe daigremontiana Raym.-Hamet \& H. } \\
\text { Perrier }\end{array}$} & Ferrara & 1970 & 1970 & $\begin{array}{l}\text { syn. Bryophyllum } \\
\text { daigremontianum }\end{array}$ \\
\hline & Bergen & $1972 \& 1977$ & $1979 \& 1978$ & \\
\hline & nn & $\begin{array}{l}\text { in collection } \\
\text { in } 1961 \& \\
1974\end{array}$ & 1966 \& 1979 & from cuttings (1961) \\
\hline \multirow[t]{2}{*}{ Kalanchoe delagoensis Eckl. \& Zeyh. } & Berlin & 1995 & 2000 & $\begin{array}{l}\text { arrived as K. tubiflora; one } \\
\text { of } 5+\text { synonyms }\end{array}$ \\
\hline & $\mathrm{nn}$ & 2001 & 2001 & \\
\hline \multirow{5}{*}{ Kalanchoe faustii Font Quer } & Muenchen & 1977 & 1980 & \\
\hline & Thorn & 1960 & 1966 & 2 synonyms \\
\hline & Duisburg & 1989 & 2000 & \\
\hline & Nice & 1997 & 2000 & \\
\hline & Essen & 2006 & no data & \\
\hline \multirow[t]{7}{*}{ Kalanchoe glaucescens Britten } & nn & unknown & 1961 & $\begin{array}{l}\text { arrived as K. flammea; one } \\
\text { of } 5 \text { synonyms }\end{array}$ \\
\hline & Palermo & 1995 & 2000 & \\
\hline & Antwerpen & 1966 & 1968 & $\begin{array}{l}\text { arrived as K. laciniata; } 5 \\
\text { synonyms }\end{array}$ \\
\hline & Amsterdam & 1967 & 1980 & \\
\hline & nn & 1965 & 1983 & \\
\hline & Berlin & 1991 & 2000 & \\
\hline & Essen & 2006 & 2006 & \\
\hline \multirow[t]{4}{*}{ Kalanchoe globulifera H. Perrier } & nn & 1952 & no data & \\
\hline & Linz & 1954 & 1961 & \\
\hline & Berlin & 1971 & 1971 & \\
\hline & Basel & 1989 & 2000 & \\
\hline
\end{tabular}


S56 Sandev, D. et al.: Plethora of plants - collections of the Botanical Garden, Faculty of Science, University of Zagreb (2)

\begin{tabular}{|c|c|c|c|c|}
\hline Scientific name & $\begin{array}{l}\text { City of plant } \\
\text { material origin }\end{array}$ & $\begin{array}{l}\text { Year of } \\
\text { ordering/ } \\
\text { purchasing }\end{array}$ & $\begin{array}{l}\text { Year when plant } \\
\text { was last recorded } \\
\text { in collection }\end{array}$ & Notes and remarks \\
\hline Kalanchoe gracilipes (Baker) Baill. & Stockholm & 1964 & 1966 & 2 synonyms \\
\hline Kalanchoe hildebrandtii Baill. & Wageningen & $1965 \& 1970$ & $1971 \& 1970$ & 3 synonyms \\
\hline Kalanchoe hybrida Desf. ex Steud. X hort. & Nancy & 1971 & 1971 & \\
\hline \multirow[t]{2}{*}{ Kalanchoe $x$ kewensis Dyer } & Antwerpen & $\begin{array}{l}1965 \& 1966 \\
\& 1967\end{array}$ & $\begin{array}{l}1971 \& 1968 \& \text { \& } \\
1971\end{array}$ & $\begin{array}{l}\text { from cuttings (1967); K. } \\
\text { flammea } \times \text { K. bentii }\end{array}$ \\
\hline & Gent & 1967 & 1979 & \\
\hline \multirow[t]{2}{*}{ Kalanchoe lanceolata (Forssk.) Pers. } & Gent & 1966 & 1971 & 2 synonyms \\
\hline & Basel & 1989 & 1990 & \\
\hline \multirow[t]{5}{*}{ Kalanchoe lateritia Engl. } & Berlin & 1965 & 1981 & $\begin{array}{l}\text { arrived as K. zimbabwensis; } \\
15+\text { synonyms }\end{array}$ \\
\hline & Antwerpen & 1967 & 1971 & from cuttings \\
\hline & nn & 1974 & 1982 & \\
\hline & Berlin & $1965 \& 1970$ & $2000 \& 1970$ & \\
\hline & Basel & 1986 & 1990 & \\
\hline \multirow[t]{3}{*}{ Kalanchoe laxiflora Baker } & nn & unknown & 1961 & 2 synonyms \\
\hline & Muenchen & 1977 & 1980 & \\
\hline & Meise & 1986 & 1988 & \\
\hline Kalanchoe lindmanii Raym.-Hamet & Antwerpen & 1967 & 1975 & $\begin{array}{l}\text { arrived as K. humbertii; } \\
\text { from cuttings }\end{array}$ \\
\hline \multirow[t]{3}{*}{ Kalanchoe longiflora Schltr. ex J. M. Wood } & Antwerpen & $\begin{array}{l}1960 \& 1965 \\
\& 1966\end{array}$ & $\begin{array}{l}1989 \& 1971 \& \\
2003\end{array}$ & \\
\hline & nn & $\begin{array}{l}\text { in collection } \\
\text { in } 1968\end{array}$ & 1971 & \\
\hline & nn & 1967 & 1970 & \\
\hline \multirow[t]{4}{*}{ Kalanchoe manginii Raym.-Hamet \& H. Perrier } & Wageningen & $\begin{array}{l}1965 \& 1966 \\
\& 1967\end{array}$ & $\begin{array}{l}1965 \& 1967 \& \\
1968\end{array}$ & $\begin{array}{l}\text { syn. K. manginii } \\
\text { var. triploidea; from } \\
\text { cuttings (1967) }\end{array}$ \\
\hline & Antwerpen & $1967 \& 1968$ & $1976 \& 1968$ & \\
\hline & Borgin & 1973 & 2010 & \\
\hline & $\mathrm{nn}$ & 1974 & 2010 & \\
\hline \multirow[t]{2}{*}{ Kalanchoe marnieriana H. Jacobsen } & Wageningen & 1965 & 1966 & 2 synonyms \\
\hline & nn & $\begin{array}{l}\text { in collection } \\
\text { in } 1968\end{array}$ & 1979 & \\
\hline Kalanchoe orgyalis Baker & Antwerpen & $\begin{array}{l}1965 \& 1966 \\
\& 1967\end{array}$ & $\begin{array}{l}1966 \& 1968 \& \text { \& } \\
1968\end{array}$ & syn. $K$. antanosiana \\
\hline Kalanchoe peteri Werderm. & Antwerpen & 1965 & 1966 & \\
\hline \multirow[t]{2}{*}{ Kalanchoe pinnata (Lam.) Pers. } & Genova & 1967 & 1980 & 4 synonyms; from cuttings \\
\hline & Rotterdam & 1969 & 1976 & \\
\hline Kalanchoe prittwitzii Engl. & Duisburg & 1995 & 2002 & $\begin{array}{l}\text { arrived as K. lugardii; one } \\
\text { of } 3 \text { synonyms }\end{array}$ \\
\hline Kalanchoe pubescens Baker & Antwerpen & $\begin{array}{l}1965 \& 1967 \\
\& 1970\end{array}$ & $\begin{array}{l}1971 \& 1971 \& \\
1979\end{array}$ & syn. Bryophyllum pubescens \\
\hline \multirow[t]{5}{*}{ Kalanchoe pumila Baker } & Wageningen & $\begin{array}{l}1965 \& 1968 \\
\& 1970\end{array}$ & $\begin{array}{l}1966 \& 2010 \& \text { \& } \\
2010\end{array}$ & 3 synonyms \\
\hline & nn & 1968 & 1982 & \\
\hline & Genova & 1967 & 1971 & \\
\hline & nn & unknown & 1971 & \\
\hline & Caen & 1993 & 2004 & \\
\hline \multirow[t]{2}{*}{ Kalanchoe rhombopilosa Mannoni \& Boiteau } & Wageningen & 1965 & 1971 & $\begin{array}{l}\text { syn. K. rhombopilosa var. } \\
\text { argentea }\end{array}$ \\
\hline & Bologna & 1966 & 1966 & \\
\hline \multirow[t]{6}{*}{ Kalanchoe rotundifolia (Haw.) Haw. } & Nancy & 1971 & 1979 & $\begin{array}{l}\text { arrived as K. guillauminii; } \\
\text { one of } 3 \text { synonyms }\end{array}$ \\
\hline & nn & 1974 & 1978 & \\
\hline & Basel & 1989 & 1990 & \\
\hline & Amsterdam & 1965 & 1965 & arrived as $K$. rotundifolia \\
\hline & Brno & 1963 & 1966 & \\
\hline & nn & $\begin{array}{l}\text { in collection } \\
\text { in } 1968\end{array}$ & 1980 & \\
\hline Kalanchoe schimperiana A. Rich. & Antwerpen & $\begin{array}{l}1965 \& 1970 \\
\& 1972\end{array}$ & $\begin{array}{l}1988 \& 1970 \& \\
1972\end{array}$ & 2 synonyms \\
\hline \multirow[t]{2}{*}{ Kalanchoe schizophylla (Baker) Baill. } & Antwerpen & $1965 \& 1971$ & $1966 \& 1971$ & 2 synonyms \\
\hline & Szeged & 1970 & 1971 & \\
\hline \multirow[t]{2}{*}{ Kalanchoe schumacheri Koord. } & Antwerpen & 1965 & 1971 & \\
\hline & nn & 1974 & 1978 & \\
\hline
\end{tabular}




\begin{tabular}{|c|c|c|c|c|}
\hline Scientific name & $\begin{array}{l}\text { City of plant } \\
\text { material origin }\end{array}$ & $\begin{array}{l}\text { Year of } \\
\text { ordering/ } \\
\text { purchasing }\end{array}$ & $\begin{array}{l}\text { Year when plant } \\
\text { was last recorded } \\
\text { in collection }\end{array}$ & Notes and remarks \\
\hline & Gent & 1979 & 1988 & \\
\hline Kalanchoe somaliensis Hook. f. & Muenchen & 1977 & 2000 & \\
\hline \multirow[t]{3}{*}{ Kalanchoe tetraphylla H. Perrier } & Antwerpen & 1965 & 1965 & \\
\hline & Edmonton & 1969 & 1971 & \\
\hline & nn & unknown & 1968 & \\
\hline Kalanchoe thyrsiflora Harv. & $\begin{array}{l}\text { Muenchen- } \\
\text { Nymphenburg }\end{array}$ & 1997 & 2000 & syn. K. alternans auct. \\
\hline \multirow[t]{3}{*}{ Kalanchoe tomentosa Baker } & Antwerpen & $\begin{array}{l}1964 \& 1965 \\
\& 1967 \& \\
1972\end{array}$ & $\begin{array}{l}1981 \& 1966 \& \& \\
1968 \& 1972\end{array}$ & from cuttings (1967) \\
\hline & Wageningen & $\begin{array}{l}1964 \& \text { \& } 1965 \\
\& 1966 \& \\
1972\end{array}$ & $\begin{array}{l}1982 \& 1966 \& \& \\
1966 \& 1972\end{array}$ & \\
\hline & Brno & 1963 & 1981 & \\
\hline \multirow{2}{*}{ Kalanchoe uniflora (Stapf) Raym.-Hamet } & Uppsala & 1970 & 1971 & 2 synonyms \\
\hline & Stockholm & 1989 & 1993 & \\
\hline Kalanchoe velutina Welw. ex Britten & Coimbra & 1984 & 1993 & 3 synonyms \\
\hline \multirow[t]{3}{*}{ Lenophyllum pusillum Rose } & Wageningen & $1955 \& 1980$ & $1961 \& 1980$ & from cuttings (1955) \\
\hline & nn & $\begin{array}{l}\text { in connection } \\
\text { in } 1963\end{array}$ & 1968 & \\
\hline & Szeged & 2000 & 2000 & \\
\hline Lenophyllum texanum (J.G. Sm.) Rose & Uppsala & 1955 & 1961 & 2 synonyms \\
\hline \multirow[t]{2}{*}{ Monanthes atlantica J. Ball } & Uppsala & & 1961 & $\begin{array}{l}\text { arrived as M. muralis; one } \\
\text { of } 5+\text { synonyms }\end{array}$ \\
\hline & Wageningen & $\begin{array}{l}1967 \& 1968 \\
\& 1970 \& \text { in } \\
\text { coll. in } 1974\end{array}$ & $\begin{array}{l}2010 \& 2010 \& \& \\
1990 \& 1979\end{array}$ & from cuttings (1967) \\
\hline \multirow[t]{2}{*}{ Monanthes laxiflora (DC.) Bolle ex Bornmuller } & nn & $\begin{array}{l}\text { in collection } \\
\text { in } 1963\end{array}$ & 1990 & 3 synonyms \\
\hline & Wageningen & $1967 \& 1970$ & $1982 \& 1982$ & from cuttings (1967) \\
\hline \multirow[t]{2}{*}{ Monanthes polyphylla (Aiton) Haw. } & Wageningen & $\begin{array}{l}1968 \& 1970 \\
\& 1971 \& \\
1972\end{array}$ & $\begin{array}{l}1971 \& 1970 \& \\
1971 \& 1972\end{array}$ & 3 synonyms \\
\hline & Besancon & 1971 & 1971 & \\
\hline Orostachys japonica (Maxim.) A. Berger & Lokrum & 1970 & 1978 & syn. Cotyledon japonica \\
\hline \multirow[t]{2}{*}{$\begin{array}{l}\text { Orostachys malacophylla (Pall.) Fisch. var. iwarenge } \\
\text { (Makino) H. Ohba }\end{array}$} & Wageningen & $1964 \& 1965$ & $1969 \& 1968$ & arrived as $O$. iwarenge \\
\hline & nn & 1964 & 1968 & \\
\hline Orostachys spinosa (L.) Sweet & Prague & 1964 & 1964 & from cuttings; 4 synonyms \\
\hline Pachyphytum bracteosum Klotzsch & Besancon & 1964 & 1977 & \\
\hline \multirow[t]{4}{*}{ Pachyphytum brevifolium Rose } & Berlin & 1958 & 1968 & \\
\hline & nn & $\begin{array}{l}\text { in collection } \\
\text { in } 1966\end{array}$ & 1979 & \\
\hline & Besancon & 1963 & 1980 & \\
\hline & Barcelona & 1965 & 1968 & \\
\hline Pachyphytum compactum Rose & nn & unknown & 1964 & $\begin{array}{l}\text { arrived as } P \text {. compactum } \\
\text { var. weinbergii }\end{array}$ \\
\hline Pachyphytum compactum Rose 'Cristatum' & Besancon & 1963 & 1975 & $\begin{array}{l}\text { unknown cultivar, } \\
\text { probably P. compactum } \mathrm{f} \text {. } \\
\text { cristatus hort. }\end{array}$ \\
\hline \multirow[t]{2}{*}{ Pachyphytum hookeri (Salm-Dyck) A. Berger } & Prague & 1963 & 1988 & syn. Diotostemon hookeri \\
\hline & nn & $\begin{array}{l}\text { in collection } \\
\text { in } 1968\end{array}$ & 1988 & \\
\hline Pachyveria x glossoides Gossot. & Antwerpen & 1969 & 1978 & $\begin{array}{l}\text { unresolved name (The } \\
\text { Plant List) }\end{array}$ \\
\hline Pachyveria x pachyphytoides (De Smet) E. Walther & nn & $\begin{array}{l}\text { in collection } \\
\text { in } 1963\end{array}$ & 1980 & $\begin{array}{l}\text { Pachyphytum bracteosum } \\
\text { x Echeveria gibbiflora var. } \\
\text { metallica }\end{array}$ \\
\hline Phedimus spurius (M. Bieb.) 't Hart & Kaunas & 1970 & 1971 & $\begin{array}{l}\text { arrived as Sedum } \\
\text { involucratum }\end{array}$ \\
\hline Phedimus spurius (M. Bieb.) 't Hart 'Splendens' & Budapest & 1971 & 1971 & 4 synonyms \\
\hline \multirow[t]{2}{*}{ Rhodiola algida Fisch. \& C.A. Mey. } & Muenchen & 1966 & no data & $\begin{array}{l}\text { arrived as Sedum } \\
\text { euphorbioides; one of } 2 \\
\text { synonyms }\end{array}$ \\
\hline & Paris & 1970 & 1973 & \\
\hline
\end{tabular}


S58 Sandev, D. et al.: Plethora of plants - collections of the Botanical Garden, Faculty of Science, University of Zagreb (2)

\begin{tabular}{|c|c|c|c|c|}
\hline Scientific name & $\begin{array}{l}\text { City of plant } \\
\text { material origin }\end{array}$ & $\begin{array}{l}\text { Year of } \\
\text { ordering/ } \\
\text { purchasing }\end{array}$ & $\begin{array}{l}\text { Year when plant } \\
\text { was last recorded } \\
\text { in collection }\end{array}$ & Notes and remarks \\
\hline & Amsterdam & 1971 & 1973 & \\
\hline Rhodiola wallichiana (Hook.) S.H. Fu & Lausanne & 1953 & 1961 & $\begin{array}{l}\text { arrived as Sedum crassipes; } \\
\text { one of } 4 \text { synonyms }\end{array}$ \\
\hline Rosularia pallida (Schott \& Kotschy) Stapf & Wageningen & $1965 \& 1980$ & $1968 \& 1980$ & syn. Umbilicus pallidus \\
\hline Rosularia pilosa (Fischer ex M. Bieberstein) Boriss. & Meinz & 1955 & 1961 & $\begin{array}{l}\text { arrived as Sedum pilosum; } \\
\text { one of } 2 \text { synonyms }\end{array}$ \\
\hline \multirow[t]{3}{*}{ Rosularia sedoides (Decne.) H. Ohba } & $\mathrm{nn}$ & $\begin{array}{l}\text { in collection } \\
\text { in } 1963\end{array}$ & 1978 & $\begin{array}{l}\text { arrived as Sempervivella } \\
\text { alba; one of } 5 \text { synonyms }\end{array}$ \\
\hline & $\begin{array}{l}\text { Vienna-Belve- } \\
\text { dere }\end{array}$ & 1978 & 1982 & \\
\hline & Caen & 1993 & 2000 & from cuttings \\
\hline $\begin{array}{l}\text { Rosularia sempervivum (M. Bieb.) A. Berger subsp. } \\
\text { sempervivum }\end{array}$ & Geneve & 1956 & 1961 & $\begin{array}{l}\text { arrived as Cotyledon libano- } \\
\text { tica; } 4 \text { synonyms }\end{array}$ \\
\hline Sedum adolphii Raym.-Hamet & Debrecen & 1955 & 1961 & from cuttings \\
\hline Sedum allantoides Rose & Wageningen & 1971 & 1975 & \\
\hline \multirow[t]{2}{*}{ Sedum amecamecanum Praeger } & Antwerpen & 1953 & 1961 & from cuttings \\
\hline & nn & $\begin{array}{l}\text { in collection } \\
\text { in } 1963\end{array}$ & 1980 & \\
\hline Sedum batesii Hemsl. & Berlin & 1968 & 1968 & $\begin{array}{l}\text { arrived as Altamiranoa } \\
\text { batesii; syn Altamiranoa } \\
\text { hemsleyana }\end{array}$ \\
\hline Sedum brevifolium DC. & Budapest & $\begin{array}{l}1970 \& 1971 \\
\& 1978\end{array}$ & $\begin{array}{l}1970 \& 1971 \& \\
1978\end{array}$ & 3 synonyms \\
\hline Sedum compactum Rose & Wageningen & 1965 & 1971 & from cuttings \\
\hline Sedum compressum Rose & Wageningen & 1955 & 1961 & \\
\hline Sedum confusum Hemsl. & Wageningen & 1969 & 2010 & \\
\hline Sedum dendroideum Moc. \& Sessé ex DC. & nn & $\begin{array}{l}\text { in collection } \\
\text { in } 1963\end{array}$ & 1966 & $\begin{array}{l}\text { syn. Sedum dendroideum } \\
\text { subsp. dendroideum }\end{array}$ \\
\hline \multirow[t]{6}{*}{$\begin{array}{l}\text { Sedum dendroideum subsp. praealtum (A. DC.) R.T. } \\
\text { Clausen }\end{array}$} & Debrecen & 1955 & no data & arrived as S. praealtum \\
\hline & nn & unknown & 1968 & \\
\hline & Wageningen & 1969 & 1971 & \\
\hline & Antwerpen & 1971 & 1971 & \\
\hline & Duisburg & 1989 & 1990 & \\
\hline & Wageningen & $\begin{array}{l}1963 \& 1965 \\
\& 1966\end{array}$ & $\begin{array}{l}1970 \& 1972 \& \\
1974\end{array}$ & $\begin{array}{l}\text { arrived as Aeonium } \\
\text { praealtum cristatum }\end{array}$ \\
\hline Sedum ebracteatum Moc. \& Sessé ex DC. & Wageningen & $1970 \& 1972$ & $1979 \& 1973$ & $10+$ synonyms \\
\hline Sedum furfuraceum Moran & Goeteborg & 1978 & 1978 & \\
\hline \multirow[t]{2}{*}{ Sedum goldmanii (Rose) Moran } & Wageningen & $\begin{array}{l}1964 \& 1967 \\
\& 1968\end{array}$ & $\begin{array}{l}1988 \& 1978 \& \& \\
1968\end{array}$ & $\begin{array}{l}\text { arrived as Villadia batesii; } \\
\text { one of } 2 \text { synonyms, from } \\
\text { cuttings }(1964,1967,1968)\end{array}$ \\
\hline & Salaspils & $1999 \& 2000$ & $2000 \& 2001$ & from cuttings $(1999,2000)$ \\
\hline Sedum greggii Hemsl. & Wageningen & 1980 & 1988 & syn. S. diversifolium \\
\hline Sedum griseum Praeger & Wageningen & $\begin{array}{l}1967 \& 1968 \\
\& 1970\end{array}$ & $\begin{array}{l}1968 \& 1971 \& \\
1982\end{array}$ & from cuttings \\
\hline \multirow[t]{4}{*}{ Sedum guatemalense Hemsl. } & Antwerpen & 1954 & no data & \\
\hline & nn & $\begin{array}{l}\text { in collection } \\
\text { in } 1963\end{array}$ & 1966 & \\
\hline & Besancon & 1971 & 1979 & \\
\hline & Berlin & 1989 & 2000 & \\
\hline \multirow[t]{2}{*}{ Sedum hultenii Fröd. } & nn & 1949 & no data & $\begin{array}{l}\text { syn. S. incertum; from } \\
\text { cuttings }\end{array}$ \\
\hline & Wageningen & 1969 & 1990 & \\
\hline Sedum humifusum Rose & nn & unknown & 1961 & \\
\hline Sedum lineare fa. variegatum Praeger & Budapest & 1971 & 1971 & \\
\hline Sedum lucidum R.T. Clausen & Wageningen & 1969 & 1978 & \\
\hline Sedum mexicanum Britton & Wageningen & $\begin{array}{l}1955 \& \text { in } \\
\text { collection in } \\
1963\end{array}$ & $1961 \& 1993$ & $\begin{array}{l}\text { syn. S. obtusolineare; from } \\
\text { cuttings (1955) }\end{array}$ \\
\hline \multirow[t]{3}{*}{ Sedum moranense Kunth } & Antwerpen & 1955 & no data & $\begin{array}{l}\text { arrived as S. arboreum; one } \\
\text { of } 3 \text { synonyms }\end{array}$ \\
\hline & Amsterdam & 1955 & 1961 & \\
\hline & $\mathrm{nn}$ & 1961 & 1963 & arrived as S. moranense \\
\hline
\end{tabular}




\begin{tabular}{|c|c|c|c|c|}
\hline Scientific name & $\begin{array}{l}\text { City of plant } \\
\text { material origin }\end{array}$ & $\begin{array}{l}\text { Year of } \\
\text { ordering/ } \\
\text { purchasing }\end{array}$ & \begin{tabular}{|l|} 
Year when plant \\
was last recorded \\
in collection
\end{tabular} & Notes and remarks \\
\hline Sedum moranense subsp. grandiflorum R.T. Clausen & Wageningen & 1971 & 1971 & $\begin{array}{l}\text { arrived as S. moranense } \\
\text { 'Grandiflorum' }\end{array}$ \\
\hline \multirow[t]{2}{*}{ Sedum morganianum E.Walther } & nn & $\begin{array}{l}\text { in collection } \\
\text { in } 1963\end{array}$ & 1968 & \\
\hline & nn & $\begin{array}{l}\text { in collection } \\
\text { in } 2000\end{array}$ & 2003 & \\
\hline \multirow[t]{2}{*}{ Sedum nussbaumerianum Bitter } & Wageningen & 1962 & 1966 & \\
\hline & Genova & 1963 & 1966 & \\
\hline \multirow[t]{2}{*}{ Sedum oaxacanum Rose } & Wageningen & 1955 & no data & \\
\hline & Wageningen & $\begin{array}{l}\text { in collection } \\
\text { in } 1963\end{array}$ & 1980 & \\
\hline Sedum oxypetalum Kunth & nn & $\begin{array}{l}\text { in collection } \\
\text { in } 1963\end{array}$ & 1966 & 2 synonyms \\
\hline Sedum pachyphyllum Rose & Wageningen & $1955 \& 1971$ & $1966 \& 1975$ & \\
\hline \multirow[t]{3}{*}{ Sedum palmeri S. Watson } & Lausanne & 1953 & 1961 & \\
\hline & Besancon & $1968 \& 1976$ & $1979 \& 1979$ & \\
\hline & Kiel & 1989 & 1993 & \\
\hline Sedum potosinum Rose & nn & 1952 & 1968 & \\
\hline Sedum pulvinatum R.T. Clausen & Wageningen & 1969 & 1977 & \\
\hline Sedum retusum Hemsl. & Uppsala & 1958 & 1975 & \\
\hline Sedum rhodicarpum Rose & Uppsala & 1958 & 1971 & \\
\hline \multirow[t]{3}{*}{ Sedum rubrotinctum R.T. Clausen } & nn & $\begin{array}{l}\text { in collection } \\
\text { in } 1961\end{array}$ & 1966 & \\
\hline & Ferara & 1970 & 1971 & \\
\hline & Wageningen & 1970 & 1971 & \\
\hline Sedum rubrotinctum R. T. Clausen cv. 'Aurora' & nn & $\begin{array}{l}\text { in collection } \\
\text { in } 1968\end{array}$ & 1971 & \\
\hline \multirow[t]{2}{*}{ Sedum spathulifolium Hook. } & nn & 1952 & no data & syn. Gormania spathulifolia \\
\hline & $\begin{array}{l}\text { Vancouver } \\
\text { (Oregon) }\end{array}$ & 1968 & 1970 & \\
\hline $\begin{array}{l}\text { Sedum spathulifolium var. pruinosum (Britton) B. } \\
\text { Boivin }\end{array}$ & Essen & 1970 & 1970 & $\begin{array}{l}\text { arrived as S. spathulifolium } \\
\text { var. purpureum }\end{array}$ \\
\hline Sedum stahlii Solms & nn & $\begin{array}{l}\text { in collection } \\
\text { in } 1963\end{array}$ & 1978 & \\
\hline \multirow[t]{3}{*}{ Sedum treleasei Rose } & Rome & 1970 & 1983 & \\
\hline & Wageningen & $1969 \& 1971$ & $1979 \& 1981$ & \\
\hline & nn & $\begin{array}{l}\text { in collection } \\
\text { in } 1975\end{array}$ & 1979 & \\
\hline Sedum urvillei DC. & Budapest & 1971 & 1961 & $\begin{array}{l}\text { arrived as S. ponticum; one } \\
\text { of } 4 \text { synonyms }\end{array}$ \\
\hline Sempervivum $\times$ funckii F.Braun ex W.D.J.Koch & Wageningen & 1956 & 1961 & $\begin{array}{l}\text { hybrid between } S \text {. } \\
\text { arachnoideum } \times S \text {. } \\
\text { montanum } \times \text { S. tectorum }\end{array}$ \\
\hline $\begin{array}{l}\text { Sempervivum montanum L. subsp. stiriacum } \\
\text { (Wettst. ex Hayek) Wettst. ex Hayek }\end{array}$ & Rotterdam & 1955 & 1961 & $\begin{array}{l}\text { arrived as S. montanum } \\
\text { var. braunii; one of } 2 \\
\text { synonyms }\end{array}$ \\
\hline Sinocrassula densirosulata (Praeger) A. Berger & nn & 1971 & 1977 & $\begin{array}{l}\text { syn. Sedum indicum var. } \\
\text { densirosulatum }\end{array}$ \\
\hline Sinocrassula yunnanensis (Franch.) A. Berger & Centonspark & 1954 & 1980 & $\begin{array}{l}\text { syn. Sedum indicum var. } \\
\text { yunnanense }\end{array}$ \\
\hline Umbilicus intermedius Boiss. & Berlin & 1978 & 1979 & 5 synonyms \\
\hline Umbilicus luteus (Huds.) Webb \& Berthel. & Genova & 1975 & 1976 & $\begin{array}{l}\text { arrived as U. erectus; one } \\
\text { of } 10 \text { synonyms }\end{array}$ \\
\hline \multirow[t]{5}{*}{ Umbilicus rupestris (Salisb.) Dandy } & Coimbra & 1970 & 1980 & $15+$ synonyms \\
\hline & Meyrin & 1976 & 1983 & \\
\hline & Antwerpen & 1955 & no data & $\begin{array}{l}\text { arrived as Cotyledon } \\
\text { umbilicus }\end{array}$ \\
\hline & Porto & 1963 & 1964 & \\
\hline & Paris & 1963 & 1965 & \\
\hline \multirow[t]{2}{*}{ Villadia batesii (Hemsl.) Baehni \& Macbride } & Wageningen & $\begin{array}{l}1964 \& 1967 \\
\& 1968\end{array}$ & $\begin{array}{l}1988 \& 1978 \& \text { \& } \\
1968\end{array}$ & $\begin{array}{l}2 \text { synonyms, from cuttings } \\
(1964,1967,1968)\end{array}$ \\
\hline & Salaspils & $1999 \& 2000$ & $2000 \& 2001$ & from cuttings $(1999,2000)$ \\
\hline \multirow[t]{2}{*}{ Villadia imbricata Rose } & Wageningen & 1957 & 1978 & 2 synonyms \\
\hline & Berlin & 1970 & 1977 & \\
\hline
\end{tabular}


S60 Sandev, D. et al.: Plethora of plants - collections of the Botanical Garden, Faculty of Science, University of Zagreb (2)

\begin{tabular}{|c|c|c|c|c|}
\hline Scientific name & $\begin{array}{l}\text { City of plant } \\
\text { material origin }\end{array}$ & $\begin{array}{l}\text { Year of } \\
\text { ordering/ } \\
\text { purchasing }\end{array}$ & $\begin{array}{l}\text { Year when plant } \\
\text { was last recorded } \\
\text { in collection }\end{array}$ & Notes and remarks \\
\hline \multicolumn{5}{|l|}{ Euphorbiaceae } \\
\hline Euphorbia abyssinica J.F.Gmel. & Palermo & $1966 \& 1978$ & 1978 \& 1980 & $\begin{array}{l}\text { arrived as E. erythraeae; } \\
\text { syn. E. abyssinica var. } \\
\text { erythraeae }\end{array}$ \\
\hline Euphorbia aggregata A.Berger & Vienna & 1964 & 1993 & \\
\hline Euphorbia alcicornis Baker & nn & $\begin{array}{l}\text { in collection } \\
\text { in } 1961\end{array}$ & 2010 & \\
\hline Euphorbia altissima Boiss. & Coimbra & 1973 & 1978 & syn. Tithymalus altissimus \\
\hline $\begin{array}{l}\text { Euphorbia amygdaloides L. subsp. robbiae (Turrill) } \\
\text { Stace }\end{array}$ & Linz & 1974 & 1977 & $\begin{array}{l}\text { arrived as E. robbiae; one } \\
\text { of } 2 \text { synonyms }\end{array}$ \\
\hline Euphorbia antiquorum L. & Sofija & 1973 & 1993 & syn. Tithymalus antiquorum \\
\hline \multirow[t]{4}{*}{ Euphorbia aphylla Brouss. ex Willd. } & Berlin & $1953 \& 1961$ & $1961 \& 1966$ & $\begin{array}{l}2 \text { synonyms; from cuttings } \\
(1953)\end{array}$ \\
\hline & Amsterdam & 1961 & 1968 & \\
\hline & Vacratot & 1971 & 1978 & \\
\hline & Antwerpen & 1973 & 1978 & \\
\hline \multirow[t]{3}{*}{ Euphorbia balsamifera Aiton } & Gent & $1961 \& 1962$ & $1961 \& 1963$ & 8 synonyms \\
\hline & Lisbon & 1960 & 1963 & \\
\hline & nn & $\begin{array}{l}\text { in collection } \\
\text { in } 1968\end{array}$ & 1976 & \\
\hline Euphorbia $\times$ bothae Lotsy \& Goddijn & Barcelona & 1970 & 1979 & $\begin{array}{l}\text { extremely variable - } 6 \\
\text { possible parents (E. } \\
\text { caerulescens, E. curvirama, E. } \\
\text { franckiana, E. ledienii, E. } \\
\text { tetragona and E. triangularis) }\end{array}$ \\
\hline Euphorbia bubalina Boiss. & nn & $1953 \& 1980$ & $1961 \& 1988$ & syn. E. laxiflora \\
\hline Euphorbia bupleurifolia Jacq. & Lokrum & 1967 & 1968 & 2 synonyms \\
\hline \multirow[t]{3}{*}{ Euphorbia canariensis L. } & nn & $\begin{array}{l}1951 \& \text { in } \\
\text { collection in } \\
1961 \& 1978\end{array}$ & $\begin{array}{l}\text { no data \& } 1978 \\
\& 1993\end{array}$ & 5 synonyms \\
\hline & Barcelona & $1970 \& 1984$ & $1978 \& 1993$ & \\
\hline & Cordoba & 1984 & 2000 & \\
\hline \multirow[t]{2}{*}{ Euphorbia candelabrum Trémaux ex Kotschy } & Amsterdam & 1993 & 1993 & $\begin{array}{l}5 \text { synonyms; from cuttings } \\
\text { gift from Orepić }\end{array}$ \\
\hline & Monaco & 1996 & 2000 & \\
\hline \multirow[t]{3}{*}{ Euphorbia caput-medusae L. } & nn & 1950 & 1979 & \\
\hline & $\mathrm{nn}$ & 1972 & 1990 & \\
\hline & Barcelona & $1970 \& 1971$ & 1993 \& 1988 & $\begin{array}{l}\text { arrived as E. caput-medusae } \\
\text { var. cristata }\end{array}$ \\
\hline Euphorbia caput-medusae L. 'Cristata' & Gent & 1960 & 1962 & \\
\hline Euphorbia ceratocarpa Ten. & Palermo & $\begin{array}{l}1966 \& 1969 \\
\& 1973\end{array}$ & $\begin{array}{l}\text { no data \& } 1971 \\
\& 1988\end{array}$ & $\begin{array}{l}\text { syn. Tithymalus } \\
\text { ceratocarpus }\end{array}$ \\
\hline Euphorbia cereiformis L. & Szeged & 1958 & 1968 & 3 synonyms \\
\hline Euphorbia clandestina Jacq. & nn & 1952 & no data & \\
\hline \multirow{2}{*}{ Euphorbia coerulescens Haw. } & Besancon & 1964 & 1966 & \\
\hline & Barcelona & 1971 & 1993 & \\
\hline Euphorbia cotinifolia L. & Muenchen & 1977 & 1988 & 3 synonyms \\
\hline Euphorbia dregeana E.Mey. ex Boiss. & Kiel & $1968 \& 1972$ & $1968 \& 1975$ & syn. Tirucalia dregeana \\
\hline Euphorbia fimbriata Scop. & Vienna & 1964 & 1968 & 4 synonyms; planta viva \\
\hline Euphorbia forsskalii J.Gay & Kirstenbosch & 1957 & 1968 & $\begin{array}{l}\text { arrived as E. burmanniana; } \\
\text { one of } 2 \text { synonyms }\end{array}$ \\
\hline Euphorbia franckiana A.Berger & Barcelona & $\begin{array}{l}1967 \& 1970 \\
\& 1971 \& \\
1972 \& 1973\end{array}$ & $\begin{array}{l}1970 \& 1978 \& \\
1988 \& 1993 \& \& \\
1974\end{array}$ & \\
\hline \multirow[t]{10}{*}{ Euphorbia globosa (Haw.) Sims } & Kiev & 1984 & 2000 & 2 synonyms \\
\hline & Szeged & 1994 & 2016 & \\
\hline & Utrecht & 1954 & no data & from cuttings \\
\hline & nn & 1952 & 1978 & \\
\hline & Genova & 1959 & 1961 & \\
\hline & Gent & 1962 & 1968 & \\
\hline & Bukurešt & 1963 & 1970 & \\
\hline & Bologna & 1973 & 1980 & \\
\hline & Bergen & $1975 \& 1977$ & $1980 \& 1982$ & \\
\hline & Jibou & 1996 & 2000 & \\
\hline
\end{tabular}




\begin{tabular}{|c|c|c|c|c|}
\hline Scientific name & $\begin{array}{l}\text { City of plant } \\
\text { material origin }\end{array}$ & $\begin{array}{l}\text { Year of } \\
\text { ordering/ } \\
\text { purchasing }\end{array}$ & $\begin{array}{l}\text { Year when plant } \\
\text { was last recorded } \\
\text { in collection }\end{array}$ & Notes and remarks \\
\hline Euphorbia grandidens Haw. & nn & $\begin{array}{l}\text { in collection } \\
\text { in } 1961\end{array}$ & 1993 & 2 synonyms; from cuttings \\
\hline Euphorbia heptagona L. var. dentata N.E. Br. & nn & 1964 & 1968 & syn. E. enopla var. dentata \\
\hline \multirow[t]{2}{*}{ Euphorbia heterophylla L. } & Antwerpen & unknown & 1961 & syn. E. cyathophora \\
\hline & Legan & $1969 \& 1970$ & $1970 \& 1970$ & \\
\hline \multirow[t]{4}{*}{ Euphorbia ledienii A.Berger } & nn & $\begin{array}{l}\text { in collection } \\
\text { in } 1984\end{array}$ & 1990 & syn. E. ledienii var. ledienii \\
\hline & Helsinki & 1958 & 1961 & \\
\hline & Bergen & unknown & 1970 & \\
\hline & Besancon & 1971 & 1978 & \\
\hline Euphorbia lignosa Marloth & Muenchen & 1978 & 1980 & syn. Tirucalia lignosa \\
\hline \multirow[t]{2}{*}{ Euphorbia lophogona Lam. } & Koeln & 1960 & 1961 & 2 synonyms \\
\hline & Uppsala & 1959 & 1963 & \\
\hline Euphorbia mammillaris L. & Rouen & 1958 & 1961 & \\
\hline \multirow[t]{6}{*}{ Euphorbia mauritanica L. } & nn & 1952 & 1961 & 2 synonyms \\
\hline & Dijon & 1957 & no data & \\
\hline & Coimbra & 1961 & 1968 & \\
\hline & Szeged & 1971 & 1971 & \\
\hline & Besancon & 1971 & 1979 & \\
\hline & Kirstenbosch & 1986 & 1988 & \\
\hline \multirow[t]{5}{*}{ Euphorbia meloformis Aiton } & nn & 1951 & no data & from cuttings; 5 synonyms \\
\hline & Vienna & 1964 & 1968 & from cuttings \\
\hline & Muenchen & 1992 & 2000 & \\
\hline & Reading & 1994 & 2000 & \\
\hline & Chemnitz & 1996 & 2000 & \\
\hline \multirow[t]{2}{*}{ Euphorbia milii Des Moul. } & Genova & 1967 & 1968 & $10+$ synonyms \\
\hline & Haren & unknown & 1988 & $\begin{array}{l}\text { arrived as E. splendens var. } \\
\text { bojeri }\end{array}$ \\
\hline \multirow[t]{2}{*}{ Euphorbia neriifolia L. } & nn & $\begin{array}{l}\text { in collection } \\
\text { in } 1961\end{array}$ & 2000 & 2 synonyms \\
\hline & nn & 1969 & 1978 & \\
\hline \multirow[t]{7}{*}{ Euphorbia obesa Hook.f. } & Vienna & $1962 \& 1963$ & $1968 \& 1968$ & syn. E. obesa subsp. obesa \\
\hline & Goettingen & 1970 & 1975 & \\
\hline & Marburg & $1991 \& 1998$ & no data \& 2000 & \\
\hline & Palermo & 1993 & 1995 & \\
\hline & Kiel & 1995 & 2010 & \\
\hline & Reading & 2002 & 2006 & \\
\hline & $\mathrm{nn}$ & unknown & 1968 & \\
\hline \multirow[t]{4}{*}{$\begin{array}{l}\text { Euphorbia obtusifolia Lam. subsp. regis-jubae (Webb } \\
\text { \& Berthel.) Maire }\end{array}$} & Palermo & $1953 \& 1966$ & $1961 \& 1975$ & $\begin{array}{l}\text { arrived as E. regis-jubae; } \\
\text { one of } 4 \text { synonyms }\end{array}$ \\
\hline & nn & $\begin{array}{l}\text { in collection } \\
\text { in } 1968\end{array}$ & 2000 & \\
\hline & Tuebingen & 1972 & 1975 & \\
\hline & Cordoba & 1985 & 1988 & \\
\hline $\begin{array}{l}\text { Euphorbia officinarum L. subsp. echinus (Hook. f. } \\
\text { \& Coss.) Vindt }\end{array}$ & Barcelona & $1972 \& 1980$ & 1993 \& 1993 & arrived as E. echinus \\
\hline Euphorbia oxyphylla Boiss. & Coimbra & 1973 & 1975 & $\begin{array}{l}\text { arrived as E. broteroi; one } \\
\text { of } 2 \text { synonyms }\end{array}$ \\
\hline \multirow[t]{3}{*}{ Euphorbia pentagona Haw. } & Palermo & 1966 & 1968 & from cuttings, 2 synonyms \\
\hline & Tuebingen & 1971 & 1978 & \\
\hline & Besancon & 1971 & no data & \\
\hline Euphorbia polycephala Marloth & Tuebingen & 1971 & 1978 & \\
\hline Euphorbia procumbens Mill. 'Cristata' & Gent & 1961 & 1968 & $\begin{array}{l}\text { possible E. pugniformis } \\
\text { 'Cristata' }\end{array}$ \\
\hline Euphorbia pseudoglobosa Marloth & Budapest & 1978 & 1980 & syn. E. frickiana \\
\hline \multirow[t]{7}{*}{ Euphorbia pteroneura A.Berger } & Hengchun & $1971 \& 1976$ & $2003 \& 2000$ & 3 synonyms \\
\hline & Muenchen & 1967 & 2003 & \\
\hline & Dresden & 2000 & 2003 & \\
\hline & Monaco & 2000 & 2003 & \\
\hline & Nantes & 1998 & 2000 & \\
\hline & Cuba & 1986 & 2000 & \\
\hline & Trontheim & 1996 & 2000 & \\
\hline Euphorbia pubescens Vahl var. crispata Boiss. & Cantonspark & 1955 & 1968 & \\
\hline
\end{tabular}


S62 Sandev, D. et al.: Plethora of plants - collections of the Botanical Garden, Faculty of Science, University of Zagreb (2)

\begin{tabular}{|c|c|c|c|c|}
\hline Scientific name & $\begin{array}{l}\text { City of plant } \\
\text { material origin }\end{array}$ & $\begin{array}{l}\text { Year of } \\
\text { ordering/ } \\
\text { purchasing }\end{array}$ & $\begin{array}{l}\text { Year when plant } \\
\text { was last recorded } \\
\text { in collection }\end{array}$ & Notes and remarks \\
\hline Euphorbia pubiglans N.E.Br. & Kirstenbosch & 1970 & 1975 & \\
\hline Euphorbia pulvinata Marloth & nn & unknown & 1979 & \\
\hline \multirow[t]{3}{*}{ Euphorbia resinifera O.Berg } & nn & $1951 \& 1969$ & $1961 \& 1988$ & $\begin{array}{l}\text { syn. Tithymalus resiniferus; } \\
\text { from cuttings (1951) }\end{array}$ \\
\hline & Bologna & 1973 & 1988 & \\
\hline & Kijev & $1984 \& 1985$ & 1993 \& 1993 & \\
\hline Euphorbia royleana Boiss. & Palermo & 1966 & 1978 & syn. E. pentagona \\
\hline Euphorbia splendens Bojer ex Hook. & Haren & 1970 & 1977 & $\begin{array}{l}\text { arrived as E. milii var. } \\
\text { splendens; one of } 2 \\
\text { synonyms }\end{array}$ \\
\hline Euphorbia succulenta (Schweick.) Bruyns & Haren & 1974 & 1975 & $\begin{array}{l}\text { arrived as Monadenium } \\
\text { stapelioides; one of } 2 \\
\text { synonyms }\end{array}$ \\
\hline \multirow[t]{2}{*}{ Euphorbia terracina L. } & Berlin & $\begin{array}{l}1962 \& 1966 \\
\& 1967 \& \\
1972 \& 1973 \\
\& 1974 \& \\
1976\end{array}$ & $\begin{array}{l}1963 \& 1966 \& \\
1968 \& 1975 \& \\
1978 \& 1982 \& \\
1988\end{array}$ & arrived as E. obtusifolia \\
\hline & nn & $\begin{array}{l}\text { in collection } \\
\text { in } 1968\end{array}$ & 1993 & \\
\hline Euphorbia tirucalli L. & nn & 1953 & 1961 & $\begin{array}{l}15+\text { synonyms; from } \\
\text { cuttings }\end{array}$ \\
\hline \multirow[t]{2}{*}{$\begin{array}{l}\text { Euphorbia tithymaloides L. subsp. smallii (Millsp.) } \\
\text { V.W.Steinm. }\end{array}$} & Szeged & 1970 & 1984 & $\begin{array}{l}\text { arrived as Pedilanthus } \\
\text { smallii; one of } 3 \text { synonyms }\end{array}$ \\
\hline & Strasbourg & 1969 & 1988 & \\
\hline Euphorbia umbellata (Pax) Bruyns & nn & unknown & 2003 & $\begin{array}{l}\text { arrived as Synadenium } \\
\text { grantii; one of } 4 \text { synonyms }\end{array}$ \\
\hline \multirow[t]{4}{*}{ Euphorbia virosa Willd. } & Antwerpen & 1959 & 1966 & 4 synonyms \\
\hline & Cagliari & 1964 & 1966 & \\
\hline & $\mathrm{nn}$ & $\begin{array}{l}\text { in collection } \\
\text { in } 1968\end{array}$ & 1981 & \\
\hline & Linz & 1978 & 1988 & \\
\hline \multirow[t]{5}{*}{ Euphorbia wildii L. C. Leach } & nn & unknown & 1966 & arrived as E. monteiri \\
\hline & Erlangen & 1965 & 1968 & \\
\hline & Muenchen & 1968 & 1970 & \\
\hline & Angola & 1971 & 1982 & \\
\hline & Muenchen & $\begin{array}{l}1972 \& 1974 \\
\& 1977 \& \\
1978 \& 2000\end{array}$ & $\begin{array}{l}1993 \& 1979 \& \\
1993 \& 1993 \& \\
2000\end{array}$ & \\
\hline \multicolumn{5}{|l|}{ Geraniaceae } \\
\hline \multirow[t]{2}{*}{ Pelargonium echinatum Curtis } & Meinz & 1955 & no data & \\
\hline & Bucharest & 1971 & no data & \\
\hline Pelargonium gibbosum (L.) L'Hér. & Cantonspark & 1954 & 1961 & $\begin{array}{l}\text { syn. Geranium gibbosum; } \\
\text { from cuttings }\end{array}$ \\
\hline Pelargonium xerophyton Schltr. ex R. Knuth & Vienna & 1964 & 1964 & from cuttings \\
\hline \multicolumn{5}{|l|}{ Oxalidaceae } \\
\hline Oxalis megalorrhiza Jacq. & Wageningen & 1962 & 1971 & 2 synonyms \\
\hline \multicolumn{5}{|l|}{ Portulacaceae } \\
\hline \multirow[t]{4}{*}{ Anacampseros arachnoides (Haw.) Sims } & Wageningen & 1958 & 1961 & 3 synonyms \\
\hline & $\mathrm{nn}$ & 1955 & 1966 & \\
\hline & Berlin & 1976 & 1990 & \\
\hline & Amsterdam & 1984 & 1990 & \\
\hline Anacampseros densifolia Dinter ex Poelln. & Berlin & 1976 & 1980 & $\begin{array}{l}\text { arrived as } A \text {. densiflora, } \\
\text { unknown name }\end{array}$ \\
\hline \multirow[t]{2}{*}{ Ancampseros filamentosa Haw. Sims. } & Koeln & 1956 & 1978 & \\
\hline & Berlin & 1976 & 1980 & \\
\hline Anacampseros lanceolata (Haw.) Sweet & Nancy & 1956 & 1966 & $\begin{array}{l}\text { syn. A. lanceolata var. } \\
\text { albiflora }\end{array}$ \\
\hline \multirow[t]{5}{*}{ Anacampseros rufescens (Haw.) Sweet } & Coimbra & $1956 \& 1965$ & 1978 \& 1972 & $\begin{array}{l}\text { syn. A. arachnoides var. } \\
\text { grandiflora }\end{array}$ \\
\hline & Beograd & 1965 & 1968 & \\
\hline & Wageningen & 1974 & 1983 & \\
\hline & Berlin & 1979 & 1993 & \\
\hline & Dresden & 1980 & no data & \\
\hline
\end{tabular}




\begin{tabular}{|c|c|c|c|c|}
\hline Scientific name & $\begin{array}{l}\text { City of plant } \\
\text { material origin }\end{array}$ & $\begin{array}{l}\text { Year of } \\
\text { ordering/ } \\
\text { purchasing }\end{array}$ & $\begin{array}{l}\text { Year when plant } \\
\text { was last recorded } \\
\text { in collection }\end{array}$ & Notes and remarks \\
\hline & Duisburg & 1986 & 1993 & \\
\hline & Jibou & 1998 & 2016 & \\
\hline \multirow[t]{2}{*}{ Anacampseros telephiastrum DC. } & Frankfurt & 1956 & 1981 & 5+ synonyms \\
\hline & Berlin & 1976 & 1993 & \\
\hline Portulacaria afra Jacq. & nn & 1949 & 2000 & 6 synonyms \\
\hline \multicolumn{5}{|l|}{ Vitaceae } \\
\hline \multirow[t]{5}{*}{ Cissus cactiformis Gilg } & Cluj-Napoca & $\begin{array}{l}1961 \& 1957 \\
\& 1958\end{array}$ & $\begin{array}{l}1961 \& 1968 \& \text { \& } \\
1970\end{array}$ & \\
\hline & Antwerpen & $1966 \& 1972$ & $1968 \& 1972$ & \\
\hline & Duisburg & 1969 & 1990 & \\
\hline & Lublin & 1993 & 1996 & \\
\hline & Antwerpen & $\begin{array}{l}1966 \& 1972 \\
\& 1970\end{array}$ & $\begin{array}{l}1966 \& 1972 \& \& \\
1978\end{array}$ & $\begin{array}{l}\text { arrived as Vitis cactiformis } \\
\text { (unknown species) }\end{array}$ \\
\hline \multirow[t]{3}{*}{ Cissus quadrangularis L. } & Duisburg & 1969 & 2000 & 5 synonyms \\
\hline & Lublin & 1993 & 2000 & \\
\hline & Bucharest & 1972 & 1975 & \\
\hline Cyphostemma currorii (Hook.f.) Desc. & Kiel & 1966 & 1970 & $\begin{array}{l}\text { arrived as Cissus } \\
\text { cramerianum; one of } 8 \\
\text { synonyms }\end{array}$ \\
\hline \multicolumn{5}{|l|}{ MONOCOTYLEDONS } \\
\hline \multicolumn{5}{|l|}{ Agavaceae } \\
\hline \multirow[t]{2}{*}{ Agave americana $\mathrm{L}$. } & nn & $\begin{array}{l}\text { in collection } \\
\text { in } 1961\end{array}$ & 1978 & $10+$ synonyms \\
\hline & nn & $\begin{array}{l}\text { in collection } \\
\text { in } 1961\end{array}$ & 1993 & $\begin{array}{l}\text { arrived as } A \text {. americana } \\
\text { var. marginata aurea }\end{array}$ \\
\hline Agave americana var. medio-picta Trel. f.alba & nn & 1995 & 2000 & gift \\
\hline \multirow[t]{2}{*}{ Agave attenuata Salm-Dyck } & Palermo & $\begin{array}{l}1962 \& 1968 \\
\& 1969\end{array}$ & $\begin{array}{l}1993 \& 1990 \& \& \\
1993\end{array}$ & $5+$ synonyms \\
\hline & nn & $1951 \& 1984$ & 1993 \& 1993 & arrived as A. cernua \\
\hline Agave aurea Brandegee & $\begin{array}{l}\text { San Marino } \\
\text { (California) }\end{array}$ & $\begin{array}{l}1976 \text { \& } 1978 \\
\& \text { in } \\
\text { collection in } \\
1988\end{array}$ & $\begin{array}{l}1990 \& 1990 \& \\
1998\end{array}$ & \\
\hline Agave bracteosa S.Watson ex Engelm. & Padova & 1983 & 2016 & \\
\hline Agave dasylirioides Jacobi \& Bouch. & Amsterdam & 1971 & 1980 & 3 synonyms \\
\hline Agave ellemeetiana Jacobi & Browning & 1953 & 1975 & $\begin{array}{l}\text { homonym A. ellemeetiana } \\
\text { K. Koch }\end{array}$ \\
\hline Agave fignemontii & Vienna & 1964 & 1990 & unknown species \\
\hline \multirow[t]{4}{*}{ Agave filifera Salm-Dyck } & Reading & 1962 & 1988 & 6 synonyms \\
\hline & Barcelona & 1969 & 1980 & \\
\hline & nn & $\begin{array}{l}\text { in collection } \\
\text { in } 1988\end{array}$ & 1993 & \\
\hline & nn & $\begin{array}{l}\text { in collection } \\
\text { in } 1974\end{array}$ & 1990 & $\begin{array}{l}\text { arrived as } A . \text { filifera var. } \\
\text { filamentosa }\end{array}$ \\
\hline \multirow[t]{3}{*}{ Agave horrida Lem. ex Jacobi } & Barcelona & $1956 \& 1969$ & $1972 \& 1980$ & $\begin{array}{l}\text { arrived as A. grandidentata; } \\
8 \text { synonyms }\end{array}$ \\
\hline & Vienna & 1964 & 1977 & \\
\hline & Barcelona & 1958 & 1961 & arrived as A. horrida \\
\hline \multirow[t]{2}{*}{ Agave kerchovei Lem. } & Nantes & 1953 & 1966 & from cuttings; 7 synonyms \\
\hline & nn & unknown & 1968 & \\
\hline Agave macroacantha Zucc. var. integrifolia Trel. & Palermo & 1969 & 1980 & \\
\hline \multirow[t]{2}{*}{ Agave mitis Mart. } & Tuebingen & 1963 & 1968 & $\begin{array}{l}\text { arrived as A. micrantha; } \\
\text { one of } 10+\text { synonyms }\end{array}$ \\
\hline & nn & unknown & no data & \\
\hline \multirow[t]{2}{*}{ Agave mitis var. albidior (Salm-Dyck) B.Ullrich } & Nancy & 1962 & 1988 & $\begin{array}{l}\text { arrived as } A \text {. albicans; one } \\
\text { of } 10+\text { synonyms }\end{array}$ \\
\hline & Barcelona & 1962 & 1988 & \\
\hline \multirow[t]{2}{*}{ Agave obscura Schiede ex Schltdl. } & Lyon & $1953 \& 1955$ & $1988 \& \mathrm{nn}$ & $\begin{array}{l}\text { arrived as } A \text {. densiflora; } \\
\text { from cuttings (1953) }\end{array}$ \\
\hline & nn & $\begin{array}{l}\text { in collection } \\
\text { in } 1968\end{array}$ & 1972 & \\
\hline Agave pendula Schnittsp. & Palermo & $1968 \& 1969$ & $1988 \& 1983$ & $\begin{array}{l}\text { arrived as } A \text {. sartori; one of } \\
7 \text { synonyms }\end{array}$ \\
\hline
\end{tabular}


S64 Sandev, D. et al.: Plethora of plants - collections of the Botanical Garden, Faculty of Science, University of Zagreb (2)

\begin{tabular}{|c|c|c|c|c|}
\hline Scientific name & $\begin{array}{l}\text { City of plant } \\
\text { material origin }\end{array}$ & $\begin{array}{l}\text { Year of } \\
\text { ordering/ } \\
\text { purchasing }\end{array}$ & $\begin{array}{l}\text { Year when plant } \\
\text { was last recorded } \\
\text { in collection }\end{array}$ & Notes and remarks \\
\hline Agave salmiana Otto ex Salm-Dyck & $\begin{array}{l}\text { old plant from } \\
\text { S3, probably } 80 \\
\text { years old }\end{array}$ & 1919.? & 1999 & $10+$ synonyms \\
\hline Agave salmiana var. ferox (K.Koch) Gentry & nn & 1951 & 1972 & $\begin{array}{l}\text { arrived as } A \text {. ferox; one of } \\
3 \text { synonyms; from cuttings }\end{array}$ \\
\hline Agave schidigera Lem. & $\begin{array}{l}\text { San Marino } \\
\text { (California) }\end{array}$ & 1976 & 2000 & $5+$ synonyms \\
\hline \multirow[t]{2}{*}{ Agave shawii Engelm. } & nn & 1951 & 1980 & 4 synonyms \\
\hline & nn & $\begin{array}{l}\text { in collection } \\
\text { in } 1975\end{array}$ & 1980 & \\
\hline \multirow[t]{3}{*}{ Agave sisalana Perrine } & $\mathrm{nn}$ & 1951 & 1988 & 5 synonyms \\
\hline & Barcelona & 1963 & 1980 & \\
\hline & Palermo & $\begin{array}{l}1964 \& 1966 \\
\& 1969\end{array}$ & $\begin{array}{l}1978 \& 1993 \& \\
1980\end{array}$ & \\
\hline Agave striata Zucc. & Lokum & 1968 & 1998 & $\begin{array}{l}\text { arrived as A. echinoides; } \\
\text { one of } 10+\text { synonyms }\end{array}$ \\
\hline \multirow[t]{2}{*}{ Agave stricta Salm-Dyck } & Berlin & $1979 \& 1981$ & $1983 \& 2000$ & 5 synonyms \\
\hline & Barcelona & 1986 & 1993 & \\
\hline \multirow[t]{2}{*}{ Agave univittata Haw. } & Lyon & 1953 & 1990 & $\begin{array}{l}\text { arrived as } A . \text { heteracantha; } \\
20+\text { synonyms }\end{array}$ \\
\hline & $\mathrm{nn}$ & 1951 & 1978 & \\
\hline Agave vandervinnenii Lem. & Palermo & 1969 & 1988 & $\begin{array}{l}\text { unresolved name (The } \\
\text { Plant List) }\end{array}$ \\
\hline \multirow[t]{2}{*}{ Agave vera-cruz Mill. } & Barcelona & 1968 & 2000 & $\begin{array}{l}\text { arrived as A. lurida; one of } \\
10+\text { synonyms }\end{array}$ \\
\hline & Palermo & 1969 & 1972 & \\
\hline \multirow[t]{7}{*}{ Agave victoriae-reginae T.Moore } & Berlin & 1960 & 1980 & $\begin{array}{l}\text { arrived as A. ferdinandi- } \\
\text { regis; one of } 5+\text { synonyms }\end{array}$ \\
\hline & Olomouc & 1989 & 1990 & \\
\hline & Amsterdam & 1989 & 1990 & \\
\hline & nn & 1952 & 1968 & $\begin{array}{l}\text { arrived as A. victoriae- } \\
\text { reginae }\end{array}$ \\
\hline & Barcelona & $\begin{array}{l}1959 \& 1967 \\
\& 1968 \& \\
1969 \& 1972\end{array}$ & $\begin{array}{l}1961 \& 1978 \& \\
2010 \& 1998 \& \\
2000\end{array}$ & \\
\hline & $\begin{array}{l}\text { Erlangen- } \\
\text { Nürnberg }\end{array}$ & 1996 & 2010 & \\
\hline & Kiel & 2000 & 2010 & \\
\hline Agave viridissima Baker & nn & $\begin{array}{l}\text { in collection } \\
\text { in } 1963\end{array}$ & 1963 & $\begin{array}{l}\text { unresolved name (The } \\
\text { Plant List) }\end{array}$ \\
\hline \multirow[t]{2}{*}{ Agave vivipara $\mathrm{L}$. } & Nantes & 1953 & no dana & $\begin{array}{l}\text { arrived as A. zapupe; from } \\
\text { cuttings; one of } 30+ \\
\text { synonyms }\end{array}$ \\
\hline & Palermo & $1957 \& 1969$ & 1993 \& 1978 & \\
\hline Agave xylonacantha Salm-Dyck & Zuerich & 1955 & 1961 & 10 synonyms \\
\hline \multirow[t]{5}{*}{ Furcraea foetida (L.) Haw. } & Palermo & $1953 \& 1964$ & $1961 \& 1978$ & $\begin{array}{l}\text { from cuttings (1953), 10+ } \\
\text { synonyms }\end{array}$ \\
\hline & Szeged & 1964 & 1975 & \\
\hline & Cagliari & 1964 & 1978 & \\
\hline & nn & $\begin{array}{l}\text { in collection } \\
\text { in } 1968\end{array}$ & 1968 & \\
\hline & Tuebingen & 1972 & 2000 & \\
\hline \multirow[t]{5}{*}{$\begin{array}{l}\text { Furcraea parmentieri (Roezl ex Ortgies) García- } \\
\text { Mend. }\end{array}$} & nn & 1951 & 1961 & $\begin{array}{l}\text { arrived as } F \text {. bedinghausii; } \\
\text { from bulbs; } 4 \text { synonyms }\end{array}$ \\
\hline & Palermo & 1966 & 1974 & \\
\hline & nn & $\begin{array}{l}\text { in collection } \\
\text { in } 1968\end{array}$ & 1968 & \\
\hline & Lokrum & 1976 & 1989 & \\
\hline & Aechen & 1990 & 1993 & \\
\hline \multirow[t]{2}{*}{ Furcraea stricta Jacobi } & nn & $\begin{array}{l}\text { in collection } \\
\text { in } 1961\end{array}$ & 1961 & arrived as F. elegans \\
\hline & Montreal & $1966 \& 1989$ & $1983 \& 2000$ & \\
\hline Manfreda maculosa (Hook.) Rose & Adelaide & $\begin{array}{l}1959 \& 1964 \\
\& 1976\end{array}$ & $\begin{array}{l}2003 \& 1984 \& \\
1977\end{array}$ & 7 synonyms \\
\hline Yucca aloifolia L. & Antibes & 1961 & 1969 & $20+$ synonyms \\
\hline
\end{tabular}




\begin{tabular}{|c|c|c|c|c|}
\hline Scientific name & $\begin{array}{l}\text { City of plant } \\
\text { material origin }\end{array}$ & $\begin{array}{l}\text { Year of } \\
\text { ordering/ } \\
\text { purchasing }\end{array}$ & $\begin{array}{l}\text { Year when plant } \\
\text { was last recorded } \\
\text { in collection }\end{array}$ & Notes and remarks \\
\hline & Barcelona & 1964 & 2000 & \\
\hline & Palermo & $1964 \& 2002$ & $2001 \& 2003$ & $\begin{array}{l}\text { arrived as Y. aloifolia } \\
\text { cv.tricolor }\end{array}$ \\
\hline & nn & $\begin{array}{l}\text { in collection } \\
\text { in } 1978\end{array}$ & 2000 & \\
\hline & Antibes & 1962 & 2003 & $\begin{array}{l}\text { arrived as Y. aloifolia var. } \\
\text { variegata }\end{array}$ \\
\hline & Potsdam & 1994 & 2003 & \\
\hline & Monaco & 1994 & 2000 & \\
\hline Yucca desmetiana Baker & nn & $\begin{array}{l}\text { in collection } \\
\text { in } 1961\end{array}$ & 1968 & \\
\hline Yucca flaccida Haw. & Lisle (Bienville) & 1978 & 1989 & $\begin{array}{l}\text { arrived as Y. louisianensis, } \\
\text { one of } 2 \text { synonyms }\end{array}$ \\
\hline Yucca glauca Nutt. & Halle & 1996 & 2010 & 5 synonyms \\
\hline Yucca gloriosa L. var. recurvifolia (Salisb.) Engelm. & nn & $\begin{array}{l}\text { in collection } \\
\text { in } 1961\end{array}$ & 1961 & arrived as Y. recurvifolia \\
\hline Yucca guatemalensis Baker & Barcelona & $1968 \& 1970$ & $1980 \& 1970$ & $\begin{array}{l}\text { arrived as Y. elephantipes, } \\
\text { one of } 2 \text { synonyms }\end{array}$ \\
\hline \multicolumn{5}{|l|}{ Aloaceae } \\
\hline Aloe africana Mill. & Kirstenbosch & $1970 \& 1971$ & $1982 \& 1984$ & 8 synonyms \\
\hline \multirow{4}{*}{ Aloe arborescens Mill. } & nn & 1961 & 1990 & $15+$ synonyms \\
\hline & Coimbra & 1970 & 1978 & \\
\hline & Barcelona & 1971 & 1978 & \\
\hline & Bucharest & $1972 \& 1973$ & $1980 \& 1978$ & \\
\hline Aloe aristata Haw. & nn & 1974 & 1989 & 4 synonyms \\
\hline Aloe camperi Schweinf. f.maculata hort. & Barcelona & 1970 & 1980 & $\begin{array}{l}\text { arrived as A. eru f. } \\
\text { maculata hort. }\end{array}$ \\
\hline Aloe capitata Baker & Barcelona & 1969 & 1981 & 2 synonyms \\
\hline \multirow[t]{3}{*}{ Aloe ciliaris Haw. } & nn & $\begin{array}{l}\text { in collection } \\
\text { in } 1968\end{array}$ & 1972 & 2 synonyms \\
\hline & Barcelona & $1962 \& 1983$ & $2016 \& 1989$ & \\
\hline & Caen & 1993 & 2000 & \\
\hline $\begin{array}{l}\text { Aloe citrea (Guillaumin) L.E. Newton \& G.D. } \\
\text { Rowley }\end{array}$ & Kiel & 1996 (1997) & 2003 & $\begin{array}{l}\text { arrived as Lomatophyllum } \\
\text { citreum }\end{array}$ \\
\hline Aloe comosa Marloth \& A.Berger & Kirstenbosch & 1970 & 1980 & \\
\hline \multirow[t]{2}{*}{ Aloe ferox Mill. } & Monaco & 1996 & 2000 & $\begin{array}{l}\text { arrived as A. candelabrum; } \\
\text { one of } 10+\text { synonyms }\end{array}$ \\
\hline & Barcelona & $\begin{array}{l}1962 \& 1963 \\
\& 1967 \& \\
1986\end{array}$ & $\begin{array}{l}1978 \& 1972 \& \& \\
1984 \& 2000\end{array}$ & arrived as A. ferox \\
\hline Aloe gariepensis Pillans & Kirstenbosch & 1984 & 1990 & \\
\hline \multirow[t]{2}{*}{ Aloe grandidentata Salm-Dyck } & nn & $\begin{array}{l}\text { in collection } \\
\text { in } 1968\end{array}$ & 1978 & \\
\hline & Kirstenbosch & 1982 & 1990 & \\
\hline $\begin{array}{l}\text { Aloe greatheadii Schönland var. daryana } \\
\text { (Schönland) Glen \& D.S.Hardy }\end{array}$ & Barkley & 1973 & 1980 & $\begin{array}{l}\text { arrived as A. davyiana; one } \\
\text { of } 7 \text { synonyms }\end{array}$ \\
\hline Aloe harlana Reynolds & Barcelona & 1980 & 1989 & \\
\hline \multirow[t]{3}{*}{ Aloe hexapetala Salm-Dyck } & Muenchen & 1976 & 1993 & $\begin{array}{l}\text { arrived as A. speciosa; one } \\
\text { of } 6 \text { synonyms }\end{array}$ \\
\hline & nn & unknown & 1990 & \\
\hline & Latte & 1990 & 2003 & \\
\hline Aloe lateritia Engl. & Uppsala & 1985 & 2003 & 4 synonyms \\
\hline Aloe lateritia var. graminicola (Reynolds) S.Carter & Berlin & 1971 & 1990 & $\begin{array}{l}\text { arrived as } A \text {. graminicola; } \\
\text { one of } 2 \text { synonyms }\end{array}$ \\
\hline Aloe littoralis Baker & Potsdam & 1966 & 1978 & $\begin{array}{l}\text { arrived as A. rubrolutea; } \\
\text { one of } 3 \text { synonyms }\end{array}$ \\
\hline Aloe longibracteata Pole-Evans & Blanes & 1984 & 1990 & \\
\hline \multirow[t]{3}{*}{ Aloe longistyla Baker } & Heidelberg & 1962 & 1978 & \\
\hline & nn & 1969 & 1980 & \\
\hline & Barcelona & 1970 & 1983 & \\
\hline \multirow[t]{2}{*}{ Aloe macrocarpa Tod. } & $\mathrm{nn}$ & 1962 & 1978 & 3 synonyms \\
\hline & nn & $\begin{array}{l}\text { in collection } \\
\text { in } 1976\end{array}$ & 1978 & \\
\hline
\end{tabular}


S66 Sandev, D. et al.: Plethora of plants - collections of the Botanical Garden, Faculty of Science, University of Zagreb (2)

\begin{tabular}{|c|c|c|c|c|}
\hline Scientific name & $\begin{array}{l}\text { City of plant } \\
\text { material origin }\end{array}$ & $\begin{array}{l}\text { Year of } \\
\text { ordering/ } \\
\text { purchasing }\end{array}$ & $\begin{array}{l}\text { Year when plant } \\
\text { was last recorded } \\
\text { in collection }\end{array}$ & Notes and remarks \\
\hline \multirow[t]{6}{*}{ Aloe maculata All. } & nn & $\begin{array}{l}\text { in collection } \\
\text { in } 1962\end{array}$ & 1993 & $\begin{array}{l}\text { arrived as A. saponaria; } 15+ \\
\text { synonyms }\end{array}$ \\
\hline & Barcelona & 1986 & 2002 & \\
\hline & Kiel & 1989 & 1993 & \\
\hline & Krakow & $1961 \& 1962$ & $1971 \& 2000$ & arrived as Aloe obscura \\
\hline & nn & 1969 & 1990 & \\
\hline & Kirstenbosch & 1971 & 1993 & \\
\hline Aloe marlothii A.Berger & Heidelberg & 1962 & 1978 & $\begin{array}{l}\text { syn. A. marlothii subsp. } \\
\text { marlothii }\end{array}$ \\
\hline \multirow[t]{4}{*}{ Aloe microstigma Salm-Dyck } & Kirstenbosch & $\begin{array}{l}1957 \& 1961 \\
\& 1971\end{array}$ & $\begin{array}{l}1978 \& 1978 \& \text { \& } \\
1982\end{array}$ & 4 synonyms \\
\hline & Heidelberg & 1962 & 1978 & \\
\hline & Adelaide & 1955 & 1978 & \\
\hline & nn & 1969 & 1978 & \\
\hline Aloe peglerae Schönland & Pretoria & 1975 & 1980 & \\
\hline Aloe percrassa Tod. & Lisbon & 1954 & 1961 & $\begin{array}{l}\text { arrived as } A \text {. schimperi; } \\
\text { one of } 4 \text { synonyms }\end{array}$ \\
\hline \multirow[t]{3}{*}{ Aloe perfoliata $\mathrm{L}$. } & Barkeley & 1973 & 1993 & $\begin{array}{l}\text { arrived as } A \text {. mitriformis; } \\
\text { one of } 20+\text { synonyms }\end{array}$ \\
\hline & Muenchen & 1995 & 1996 & \\
\hline & Budapest & 1970 & 1970 & arrived as A. nobilis \\
\hline \multirow{2}{*}{ Aloe plicatilis (L.) Mill. } & Barcelona & 1970 & 1982 & $5+$ synonyms \\
\hline & Kirstenbosch & 1986 & 1993 & \\
\hline Aloe rauhii Reynolds & Wageningen & $\begin{array}{l}1967 \& 1969 \\
\& 1972\end{array}$ & $\begin{array}{l}1972 \& 1978 \& \\
2000\end{array}$ & syn. Guillauminia rauhii \\
\hline Aloe reitzii Reynolds & Barcelona & $1967 \& 1971$ & $1978 \& 1990$ & syn. $A$. reitzii var. reitzii \\
\hline Aloe $\times$ runcinata A.Berger & Lisbon & 1954 & 1961 & arrived as $A$. runcinata \\
\hline Aloe spectabilis Reynolds & Latte & 1990 & 2000 & \\
\hline Aloe straussii A.Berger & Adelaide & 1959 & 1978 & $\begin{array}{l}\text { unresolved name (The } \\
\text { Plant List) }\end{array}$ \\
\hline \multirow[t]{3}{*}{ Aloe striata Haw. } & Kirstenbosch & 1961 & 2003 & 5 synonyms \\
\hline & Antibes & 1962 & 1978 & \\
\hline & nn & $\begin{array}{l}\text { in collection } \\
\text { in } 1968\end{array}$ & 1972 & \\
\hline Aloe succotrina Lam. & Antibes & 1962 & 1978 & $\begin{array}{l}\text { arrived as A. purpurascens; } \\
\text { one of } 5+\text { synonyms }\end{array}$ \\
\hline Aloe tenuior Haw. & Barcelona & 1980 & 1983 & 5 synonyms \\
\hline Aloe thraskii Baker+B202 & Kiel & 1958 & 1963 & \\
\hline Aloe $\mathrm{x}$ todari Borzi & Barcelona & 1971 & 2003 & A. humilis $\times$ A. sp. \\
\hline Aloe vaombe Decorse \& Poisson & Essen & 2006 & 2010 & syn. A. vaombe var. vaombe \\
\hline \multirow[t]{6}{*}{ Aloe variegata $\mathrm{L}$. } & Vienna & 1964 & 1968 & 3 synonyms \\
\hline & Brisbane & 1963 & 1978 & \\
\hline & Barcelona & 1987 & 2000 & \\
\hline & Tartu & 1993 & 2000 & \\
\hline & Montreal & 1995 & 2000 & \\
\hline & Monaco & 1996 & 2002 & \\
\hline \multirow[t]{5}{*}{ Aloe vera (L.) Burm. f. } & Palermo & 1998 & 2016 & $15+$ synonyms \\
\hline & nn & 1969 & 1979 & \\
\hline & Sienne & 1990 & 1993 & \\
\hline & Tuebingen & 1995 & 1996 & \\
\hline & $\mathrm{nn}$ & 2009 & 2016 & bought in Bauhaus \\
\hline Bulbine caulescens L. & Blanes & 1997 & 2003 & $\begin{array}{l}\text { arrived as } B \text {. frutescens; } \\
\text { one of } 4 \text { synonyms }\end{array}$ \\
\hline × Gasteraloe lapaixii (Radl) Guillaumin & Palermo & 1964 & 1980 & $\begin{array}{l}\text { arrived as Aloe } \times \text { lapaixii; } \\
\text { Aloe aristata } \times \text { Gasteria } \\
\text { bicolor var. bicolor }\end{array}$ \\
\hline \multirow[t]{3}{*}{$\begin{array}{l}\times \text { Gasterhaworthia bayfieldii (Salm-Dyck) } \\
\text { G.D.Rowley }\end{array}$} & Wageningen & $\begin{array}{l}1964 \& 1965 \\
\& 1968 \& \\
1969\end{array}$ & $\begin{array}{l}1990 \& 1990 \& \\
1981 \& 2004\end{array}$ & $\begin{array}{l}\text { arrived as Aloe } \times \text { bayfieldii, } \\
\text { one of } 2 \text { synonyms; } \\
\text { Gasteria } \times \text { Haworthia }\end{array}$ \\
\hline & Wageningen & $\begin{array}{l}\text { in collection } \\
\text { in } 1975\end{array}$ & 1983 & \\
\hline & Wageningen & $\begin{array}{l}\text { in collection } \\
\text { in } 1989\end{array}$ & 2003 & \\
\hline Gasteria acinacifolia (J.Jacq.) Haw. & nn & $\begin{array}{l}\text { in collection } \\
\text { in } 1961\end{array}$ & 1961 & $20+$ synonyms \\
\hline
\end{tabular}




\begin{tabular}{|c|c|c|c|c|}
\hline Scientific name & $\begin{array}{l}\text { City of plant } \\
\text { material origin }\end{array}$ & $\begin{array}{l}\text { Year of } \\
\text { ordering/ } \\
\text { purchasing }\end{array}$ & $\begin{array}{l}\text { Year when plant } \\
\text { was last recorded } \\
\text { in collection }\end{array}$ & Notes and remarks \\
\hline & Adelaide & 1953 & 2010 & $\begin{array}{l}\text { arrived as G. acinacifolia } \\
\text { var. Venusta }\end{array}$ \\
\hline \multirow[t]{2}{*}{ Gasteria bicolor Haw. var. bicolor } & Frankurt/Mein & 1960 & 2003 & $\begin{array}{l}\text { arrived as G. maculata; } \\
\text { from cuttings; one of } 5 \\
\text { synonyms }\end{array}$ \\
\hline & Duisburg & 1998 & 2000 & \\
\hline Gasteria bicolor var. liliputana (Poelln.) Van Jaarsv. & Wageningen & $\begin{array}{l}1967 \& 1969 \\
\& 1972\end{array}$ & $\begin{array}{l}1971 \& 1971 \& \\
2000\end{array}$ & $\begin{array}{l}\text { arrived as syn. G. } \\
\text { liliputana; from cuttings } \\
\text { (1967) }\end{array}$ \\
\hline \multirow[t]{2}{*}{ Gasteria brevifolia Haw. } & nn & 1949 & 1961 & $\begin{array}{l}\text { syn. G. brevifolia var. } \\
\text { latevirens }\end{array}$ \\
\hline & nn & $\begin{array}{l}\text { in collection } \\
\text { in } 1963\end{array}$ & 1993 & \\
\hline Gasteria carinata (Mill.) Duval & nn & unknown & 1961 & $\begin{array}{l}\text { arrived as G. excavata; one } \\
\text { of } 2 \text { synonyms }\end{array}$ \\
\hline \multirow[t]{2}{*}{ Gasteria carinata var. carinata } & Berlin & 1978 & 2016 & $\begin{array}{l}\text { arrived as G. sulcata; one } \\
\text { of } 20+\text { synonyms }\end{array}$ \\
\hline & Wuppertal & 1995 & 2016 & \\
\hline $\begin{array}{l}\text { Gasteria carinata var. glabra (Salm-Dyck) van } \\
\text { Jaarsv. }\end{array}$ & Duisburg & 1995 & 2000 & $\begin{array}{l}\text { arrived as G. glabra; one of } \\
30+\text { synonyms }\end{array}$ \\
\hline \multirow[t]{3}{*}{ Gasteria carinata var. verrucosa (Mill.) Van Jaarsv. } & Adelaide & 1953 & 2003 & $\begin{array}{l}\text { arrived as G. verrucosa } \\
\text { var. asperrima; } 10+ \\
\text { synonyms }\end{array}$ \\
\hline & Adelaide & $\begin{array}{l}\text { in collection } \\
\text { in } 1968\end{array}$ & 1981 & \\
\hline & Marburg & 1953 & 1961 & arrived as G. verrucosa \\
\hline \multirow[t]{2}{*}{ Gasteria $\times$ cheilophylla Baker } & Adelaide & $\begin{array}{l}1954 \& 1955 \\
\& 1959\end{array}$ & $\begin{array}{l}1961 \& 2000 \& \\
1979\end{array}$ & G. carinata $\times$ G. pulchra \\
\hline & Besancon & 1971 & no dana & \\
\hline \multirow[t]{3}{*}{ Gasteria disticha (L.) Haw. } & Nancy & 1953 & 1961 & $\begin{array}{l}\text { arrived as G. obtusifolia; } \\
20+\text { synonyms }\end{array}$ \\
\hline & Nantes & 1953 & 1980 & \\
\hline & Berlin & 1978 & 2016 & arrived as G. conspurcata \\
\hline Gasteria excelsa Baker & Berlin & 1979 & 2010 & \\
\hline Gasteria gigantea Graessn. & Cluj-Napoca & 2000 & 2006 & (neriješen status) \\
\hline Gasteria nitida (Salm-Dyck) Haw. & nn & $\begin{array}{l}\text { in collection } \\
\text { in } 1961\end{array}$ & 1961 & 3 synonyms \\
\hline \multirow[t]{2}{*}{$\begin{array}{l}\text { Gasteria nitida var. armstrongii (Schönland) van } \\
\text { Jaarsv. }\end{array}$} & Berlin & 1978 & 1993 & $\begin{array}{l}\text { arrived as G. armstrongii; } \\
\text { one of } 2 \text { homonyms }\end{array}$ \\
\hline & Pecs & 2005 & 2009 & \\
\hline Gasteria pseudonigricans (Salm-Dyck) Haw. & Gent & 1970 & 2010 & syn. Aloe pseudonigricans \\
\hline Gasteria pulchra (Aiton) Haw. & $\mathrm{nn}$ & 1951 & 2003 & 4 synonyms \\
\hline \multirow[t]{2}{*}{ Gasteria trigona Haw. } & Besancon & 1971 & 1981 & 2 synonyms \\
\hline & Wuppertal & $1995 \& 1996$ & $2003 \& 1997$ & \\
\hline Haworthia aristata Haw. & Palermo & 1961 & 1962 & 3 synonyms \\
\hline \multirow[t]{3}{*}{ Haworthia attenuata (Haw.) Haw. } & Muenster & 1966 & 1980 & $\begin{array}{l}\text { arrived as } H \text {. tisleyi; one of } \\
2 \text { synonyms }\end{array}$ \\
\hline & Debrecen & 1956 & 1961 & arrived as H. attenuata \\
\hline & Wageningen & 1964 & 2000 & $\begin{array}{l}\text { arrived as Haworthia } \\
\text { attenuata var. argyrostigma }\end{array}$ \\
\hline $\begin{array}{l}\text { Haworthia attenuata var. britteniana (Poelln.) } \\
\text { Poelln. }\end{array}$ & Liberec & 1965 & 1980 & 2 synonyms \\
\hline \multirow[t]{4}{*}{ Haworthia coarctata Haw. } & Wageningen & $\begin{array}{l}1966 \& 1967 \\
\& 1968\end{array}$ & $\begin{array}{l}1980 \& 1983 \& \text { \& } \\
1979\end{array}$ & $\begin{array}{l}\text { arrived as H. resendeana; } \\
\text { one of } 20+\text { synonyms }\end{array}$ \\
\hline & nn & $\begin{array}{l}\text { in collection } \\
\text { in } 1961\end{array}$ & 1982 & arrived as $H$. coarctata \\
\hline & Iasi & 1963 & 1966 & \\
\hline & Wageningen & 1970 & 2001 & \\
\hline Haworthia coarctata Haw. f. coarctata & Wageningen & $1964 \& 1966$ & $1975 \& 1980$ & $\begin{array}{l}\text { arrived as H. reinwardtii } \\
\text { var. fallax; one of } 2 \\
\text { synonyms }\end{array}$ \\
\hline Haworthia coarctata f. greenii (Baker) M.B.Bayer & Wageningen & 1970 & 2000 & $\begin{array}{l}\text { arrived as H. greenii; one } \\
\text { of } 8 \text { synonyms }\end{array}$ \\
\hline
\end{tabular}


S68 Sandev, D. et al.: Plethora of plants - collections of the Botanical Garden, Faculty of Science, University of Zagreb (2)

\begin{tabular}{|c|c|c|c|c|}
\hline Scientific name & $\begin{array}{l}\text { City of plant } \\
\text { material origin }\end{array}$ & $\begin{array}{l}\text { Year of } \\
\text { ordering/ } \\
\text { purchasing }\end{array}$ & $\begin{array}{l}\text { Year when plant } \\
\text { was last recorded } \\
\text { in collection }\end{array}$ & Notes and remarks \\
\hline \multirow[t]{3}{*}{ Haworthia cooperi Baker } & Wageningen & $\begin{array}{l}1964 \& 1965 \\
\& 1972 \& \\
1974 \& 1980\end{array}$ & $\begin{array}{l}1980 \& 1980 \& \\
1980 \& 1975 \& \\
1980\end{array}$ & arrived as syn. $H$. vittata \\
\hline & nn & 1971 & 1978 & \\
\hline & Pecs & 1996 & 1997 & \\
\hline Haworthia cooperi var. tenera (Poelln.) M. B. Bayer & Brno & 1963 & 1975 & $\begin{array}{l}\text { arrived as } H \text {. tenera; one of } \\
2 \text { synonyms }\end{array}$ \\
\hline \multirow[t]{5}{*}{ Haworthia cymbiformis (Haw.) Duval } & Wageningen & 1964 & 1966 & $\begin{array}{l}\text { arrived as H. planifolia; one } \\
\text { of } 20+\text { synonyms, P.V. }\end{array}$ \\
\hline & Antwerpen & 1965 & 1971 & arrived as H. cymbiformis \\
\hline & Iasi & 1963 & 1971 & \\
\hline & Palermo & 1963 & 1990 & \\
\hline & Cluj & 1967 & 1977 & from cuttings \\
\hline Haworthia cymbiformis (Haw.) Duval cv. variegata & Wageningen & $1966 \& 1968$ & $1980 \& 1971$ & \\
\hline Haworthia fasciata (Willd.) Haw. & Palermo & 1962 & 1980 & $\begin{array}{l}\text { arrived as } H \text {. subfasciata; } \\
\text { one of } 15+\text { synonyms }\end{array}$ \\
\hline \multirow[t]{2}{*}{ Haworthia herbacea (Mill.) Stearn } & Liberec & 1964 & 1966 & $\begin{array}{l}\text { arrived as H. pallida; } 10+ \\
\text { synonyms }\end{array}$ \\
\hline & Pesc & 1996 & 2001 & arrived as $\mathrm{H}$. translucens \\
\hline Haworthia icosiphylla Baker & Wageningen & 1965 & 2000 & \\
\hline Haworthia janseneana Uitewaal & Wageningen & 1964 & 2001 & from cuttings \\
\hline \multirow{3}{*}{ Haworthia margaritifera (L.) Haw. } & nn & 1952 & 1961 & $20+$ synonyms \\
\hline & Kirstenbosch & 1961 & 1979 & \\
\hline & Pecs & 1997 & 2000 & arrived as planta viva \\
\hline Haworthia marumiana Uitewaal & Wageningen & $\begin{array}{l}1964 \& 1967 \\
\& 1968 \& \\
1970\end{array}$ & $\begin{array}{l}1979 \& 1968 \& \\
1980 \& 1980\end{array}$ & from cuttings $(1964,1967)$ \\
\hline \multirow[t]{3}{*}{$\begin{array}{l}\text { Haworthia marumiana var. batesiana (Uitewaal) M. } \\
\text { B. Bayer }\end{array}$} & Wageningen & $\begin{array}{l}1961 \& 1964 \\
\& 1965 \& \\
1972\end{array}$ & $\begin{array}{l}2001 \& 1995 \& \\
1980 \& 1975\end{array}$ & arrived as H. batesiana \\
\hline & Besancon & 1971 & 1983 & \\
\hline & Kiev & 1989 & 1990 & \\
\hline \multirow[t]{7}{*}{ Haworthia maxima (Haw.) Duval } & Valencia & $1961 \& 1968$ & $1980 \& 1971$ & $\begin{array}{l}\text { arrived as H. semimargari- } \\
\text { tifera; } 5+\text { synonyms }\end{array}$ \\
\hline & Goettingen & 2000 & 2003 & \\
\hline & Madrid & 2000 & 2003 & \\
\hline & Pecs & 1997 & 2001 & from cuttings \\
\hline & nn & $\begin{array}{l}\text { in collection } \\
\text { in } 1971\end{array}$ & 1980 & \\
\hline & Palermo & 1966 & 1980 & $\begin{array}{l}\text { arrived as H. papillosa; one } \\
\text { of } 10+\text { synonyms; from } \\
\text { cuttings }\end{array}$ \\
\hline & Pecs & 1997 & 2000 & arrived as planta viva \\
\hline $\begin{array}{l}\text { Haworthia mirabilis (Haw.) Haw. var. triebneriana } \\
\text { (Poelln.) M. B. Bayer }\end{array}$ & Wageningen & $1964 \& 1971$ & $1980 \& 1978$ & arrived as $H$. nitidula \\
\hline Haworthia mucronata Haw. & Wageningen & $1964 \& 1965$ & $1971 \& 1985$ & $\begin{array}{l}\text { arrived as } H \text {. altilinea; one } \\
\text { of } 3 \text { synonyms }\end{array}$ \\
\hline Haworthia reinwardtii (Salm-Dyck) Haw. & Kiev & 1989 & 1990 & $10+$ synonyms \\
\hline Haworthia retusa (L.) Duval & Wuppertal & 1995 & 1997 & syn. Aloe retusa \\
\hline \multirow[t]{2}{*}{ Haworthia rigida (Lam.) Haw. } & nn & unknown & 1961 & 8 synonyms \\
\hline & Wageningen & $\begin{array}{l}1964 \& 1965 \\
\& 1968\end{array}$ & $\begin{array}{l}2004 \& 1980 \& \\
2001\end{array}$ & $\begin{array}{l}\text { arrived as H. rigida var. } \\
\text { expansa }\end{array}$ \\
\hline Haworthia ryderiana Poelln. & Wageningen & $1964 \& 1965$ & $1990 \& 2000$ & $\begin{array}{l}\text { arrived as planta viva } \\
\text { (1964) }\end{array}$ \\
\hline Haworthia subattenuata (Salm-Dyck) Baker & Palermo & 1958 & 2001 & syn. Aloe subattenuata \\
\hline \multirow[t]{2}{*}{ Haworthia turgida Haw. } & Berlin & 1960 & 1961 & $\begin{array}{l}\text { arrived as H. laetevirens, } \\
\text { one of } 2 \text { synonyms }\end{array}$ \\
\hline & nn & $\begin{array}{l}1961 \& \text { in } \\
\text { collection in } \\
1968\end{array}$ & $1963 \& 1983$ & \\
\hline \multirow[t]{2}{*}{ Haworthia viscosa (L.) Haw. } & Wageningen & 1965 & 2003 & $\begin{array}{l}\text { arrived as } H . \text { tortuosa var. } \\
\text { curta; } 30+\text { synonyms }\end{array}$ \\
\hline & Wageningen & $1965 \& 1970$ & $2001 \& 2001$ & $\begin{array}{l}\text { arrived as H. tortuosa var. } \\
\text { variegata }\end{array}$ \\
\hline
\end{tabular}




\begin{tabular}{|c|c|c|c|c|}
\hline Scientific name & $\begin{array}{l}\text { City of plant } \\
\text { material origin }\end{array}$ & $\begin{array}{l}\text { Year of } \\
\text { ordering/ } \\
\text { purchasing }\end{array}$ & $\begin{array}{l}\text { Year when plant } \\
\text { was last recorded } \\
\text { in collection }\end{array}$ & Notes and remarks \\
\hline & Wageningen & 1964 & 2003 & $\begin{array}{l}\text { from cuttings; arrived as } \\
\text { H. cassytha }\end{array}$ \\
\hline & Valencia & $1961 \& 1962$ & $1980 \& 1963$ & $\begin{array}{l}\text { arrived as H. tortuosa var. } \\
\text { pseudorigida }\end{array}$ \\
\hline & Palermo & 1958 & 1968 & $\begin{array}{l}\text { arrived as H. tortuosa var. } \\
\text { pseudorigida }\end{array}$ \\
\hline & Debrecen & 1956 & 1961 & arrived $H$. tortuosa \\
\hline & Kiev & 1989 & 1990 & \\
\hline & nn & 1964 & 1971 & arrived as H. asperiuscula \\
\hline \multirow[t]{4}{*}{$\begin{array}{l}\text { Haworthiopsis attenuata (Haw.) G.D. Rowley } \\
\text { var. attenuata }\end{array}$} & Monaco & 1994 & 2001 & $\begin{array}{l}\text { arrived as Haworthia } \\
\text { attenuata var. argyrostigma; } \\
15+\text { synonyms }\end{array}$ \\
\hline & Palermo & $\begin{array}{l}1957 \& 1960 \\
\& 1961\end{array}$ & $\begin{array}{l}\text { no data \& } 2001 \\
\& 1961\end{array}$ & $\begin{array}{l}\text { arrived as Haworthia } \\
\text { attenuata var. clariperla }\end{array}$ \\
\hline & Wageningen & $1964 \& 1965$ & $1980 \& 1980$ & $\begin{array}{l}\text { from cuttings (1964); } \\
\text { arrived as Haworthia } \\
\text { subulata }\end{array}$ \\
\hline & Dijon & 1989 & 2004 & \\
\hline \multirow[t]{6}{*}{$\begin{array}{l}\text { Haworthiopsis attenuata var. radula (Jacq.) G.D. } \\
\text { Rowley }\end{array}$} & nn & 1958 & 1961 & $\begin{array}{l}\text { arrived as Haworthia } \\
\text { rugosa, } 4 \text { synonyms }\end{array}$ \\
\hline & Barcelona & 1961 & 1962 & \\
\hline & Iasi & 1958 & 2004 & \\
\hline & Palermo & 1957 & 1961 & \\
\hline & nn & $\begin{array}{l}\text { in collection } \\
\text { in } 1963\end{array}$ & 1980 & \\
\hline & Wageningen & 1969 & 1980 & \\
\hline $\begin{array}{l}\text { Haworthiopsis coarctata (Haw.) G.D. Rowley } \\
\text { var. coarctata }\end{array}$ & Wageningen & $\begin{array}{l}1966 \& 1967 \\
\& 1970\end{array}$ & $\begin{array}{l}1993 \& 1978 \& \\
2002\end{array}$ & $\begin{array}{l}\text { arrived as Haworthia } \\
\text { greenii; } 10+\text { synonyms; } \\
\text { from cuttings (1967) }\end{array}$ \\
\hline $\begin{array}{l}\text { Haworthiopsis coarctata var. tenuis (G.G. Sm.) G.D. } \\
\text { Rowley }\end{array}$ & Wageningen & 1966 & 1978 & $\begin{array}{l}\text { arrived as Haworthia } \\
\text { reinwardtii var. tenuis; one } \\
\text { of } 2 \text { synonyms }\end{array}$ \\
\hline \multirow[t]{2}{*}{ Haworthiopsis fasciata (Willd.) G.D. Rowley } & Valencia & 1961 & 1966 & $\begin{array}{l}\text { arrived as Haworthia } \\
\text { fasciata; one of } 4 \text { synonyms }\end{array}$ \\
\hline & Monaco & 1992 & 2001 & \\
\hline \multirow[t]{3}{*}{$\begin{array}{l}\text { Haworthiopsis glauca (Baker) G. D. Rowley var. } \\
\text { herrei (Poelln.) G.D. Rowley }\end{array}$} & Wageningen & $1968 \& 1970$ & $1978 \& 1978$ & $\begin{array}{l}\text { arrived as Haworthia } \\
\text { armstrongii; one of } 4 \\
\text { synonyms }\end{array}$ \\
\hline & Wageningen & $1968 \& 1969$ & $1980 \& 1979$ & arrived as Haworthia eilyae \\
\hline & Wageningen & 1969 & 1978 & arrived as Haworthia herrei \\
\hline Haworthiopsis limifolia (Marloth) G.D. Rowley & Bologna & 1966 & 1979 & $\begin{array}{l}\text { arrived as Haworthia } \\
\text { limifolia }\end{array}$ \\
\hline \multirow[t]{3}{*}{ Haworthiopsis tessellata (Haw.) G.D. Rowley } & nn & 1957 & 1961 & $\begin{array}{l}\text { arrived as Haworthia } \\
\text { tessellata; one of } 2 \\
\text { synonyms }\end{array}$ \\
\hline & Debrecen & 1956 & 1975 & \\
\hline & Vienna & 1964 & 1975 & from cuttings \\
\hline \multicolumn{5}{|l|}{ Bromeliaceae } \\
\hline \multirow[t]{5}{*}{ Dyckia brevifolia Baker } & nn & 1951 & no data & $\begin{array}{l}\text { arrived as D. sulphurea; } \\
\text { one of } 4 \text { synonyms }\end{array}$ \\
\hline & Kaunas & 1958 & 1990 & \\
\hline & Barcelona & $1981 \& 1993$ & $1991 \& 1995$ & arrived as D. brevifolia \\
\hline & nn & $\begin{array}{l}\text { in collection } \\
\text { in } 1984\end{array}$ & 1993 & \\
\hline & Liege & 1993 & 1995 & \\
\hline Dyckia rariflora Schult. \& Schult.f. & Helsinki & 1960 & 1961 & \\
\hline \multicolumn{5}{|l|}{ Hyacinthaceae } \\
\hline \multirow[t]{2}{*}{ Bowiea volubilis Harv. } & nn & $\begin{array}{l}\text { in collection } \\
\text { in } 1968\end{array}$ & 1978 & 2 synonyms \\
\hline & Kirstenbosch & 1970 & 1983 & \\
\hline
\end{tabular}


S70 Sandev, D. et al.: Plethora of plants - collections of the Botanical Garden, Faculty of Science, University of Zagreb (2)

Tab. 3. Collection of the cold glasshouse: recently existing plants (May, 2017).

\begin{tabular}{|c|c|c|c|c|c|}
\hline Scientific name & $\begin{array}{l}\text { City of plant } \\
\text { material origin }\end{array}$ & $\begin{array}{l}\text { Year of } \\
\text { ordering/ } \\
\text { purchasing }\end{array}$ & CITES & EASIN & Notes and remarks \\
\hline \multicolumn{6}{|l|}{ D ICOTYLEDONS } \\
\hline \multicolumn{6}{|l|}{ Aizoaceae } \\
\hline Aloinopsis malherbei (L. Bolus) L. Bolus & Innsbruck & 2017 & no & no & syn. Nananthus malherbei \\
\hline Aptenia cordifolia (L.f.) Schwantes & Cluj-Napoca & 2017 & no & no & 4 synonyms \\
\hline Astridia herrei L. Bolus & Pecs & 2016 & no & no & \\
\hline Bergeranthus multiceps (Salm-Dyck) Schwantes & Budapest & 2017 & no & no & $\begin{array}{l}\text { arrived as } B \text {. artus; one of } 4 \\
\text { synonyms }\end{array}$ \\
\hline Bergeranthus scapiger (Haw.) Schwantes & Berlin-Dahlem & 1976 & no & no & $\begin{array}{l}\text { syn. Mesembryanthemum } \\
\text { scapiger }\end{array}$ \\
\hline \multirow[t]{2}{*}{ Carpobrotus acinaciformis (L.) L.Bolus } & Aaechen & 1996 & no & yes & $10+$ synonyms \\
\hline & Nantes & 1998 & no & yes & \\
\hline Carruanthus peersii L. Bolus & Bochum & 2011 & no & no & \\
\hline $\begin{array}{l}\text { Chasmatophyllum masculinum (Haw.) Dinter } \\
\& \text { Schwantes }\end{array}$ & Richmond & 2000 & no & no & $\begin{array}{l}\text { syn. Mesembryanthemum } \\
\text { musculinum }\end{array}$ \\
\hline Conophytum bilobum (Marloth) N.E. Br. & Monaco & 1993 & no & no & $40+$ synonyms \\
\hline \multirow[t]{2}{*}{$\begin{array}{l}\text { Corpuscularia lehmannii (Eckl. \& Zeyh.) } \\
\text { Schwantes }\end{array}$} & nn & $\begin{array}{l}\text { in collection } \\
\text { in } 1963\end{array}$ & no & no & 4 synonyms \\
\hline & Wageningen & 1986 & no & no & \\
\hline Cylindrophyllum calamiforme (L.) Schwantes & Berkeley & 1994 & no & no & $\begin{array}{l}\text { syn. Mesembryanthemum } \\
\text { calamiforme }\end{array}$ \\
\hline Delosperma bosseranum Marais & Monaco & 2017 & no & no & \\
\hline \multirow[t]{3}{*}{ Delosperma cooperi (Hook.f.) L. Bolus } & Plzen & 2016 & no & yes & 2 synonyms \\
\hline & Vienna & 2016 & no & yes & \\
\hline & MBM - bought & unknown & no & yes & $\begin{array}{l}\text { syn. Mesembryanthemum } \\
\text { cooperi }\end{array}$ \\
\hline Delosperma echinatum (Lam.) Schwantes & Berlin-Dahlem & 2003 & no & no & 5 synonyms \\
\hline Delosperma ecklonis (Salm-Dyck) Schwantes & Berlin-Dahlem & 1972 & no & no & 2 synonyms \\
\hline \multirow[t]{2}{*}{$\begin{array}{l}\text { Delosperma lehmannii (Eckl. \& Zeyh.) } \\
\text { Schwantes ex H.Jacobsen }\end{array}$} & nn & unknown & no & no & \\
\hline & Cuba & 1986 & no & no & \\
\hline Delosperma pachyrhizum L. Bolus & Berlin-Dahlem & 1978 & no & no & $\begin{array}{l}\text { arrived as D. pachyrhizum } \\
\text { var. pubescens }\end{array}$ \\
\hline Delosperma robustum L. Bolus & Gent & 1967 & no & no & \\
\hline Delosperma sutherlandii (Hook.f.) N.E.Br. & Potsdam & 2016 & no & no & $\begin{array}{l}\text { syn. Mesembryanthemum } \\
\text { sutheralndii }\end{array}$ \\
\hline Faucaria bosscheana (A. Berger) Schwantes & Bochum & 1995 & no & no & $\begin{array}{l}\text { arrived as F. paucidens; one } \\
\text { of } 8 \text { synonyms }\end{array}$ \\
\hline Faucaria britteniae L. Bolus & Pecs & 2005 & no & no & $\begin{array}{l}\text { arrived as } F . \text { grandis; one of } \\
5 \text { synonyms }\end{array}$ \\
\hline Faucaria felina (L.) Schwantes & Cluj-Napoca & 2014 & no & no & $\begin{array}{l}\text { arrived as F. felina var. } \\
\text { jamesii; one of } 20+\text { synonyms }\end{array}$ \\
\hline Faucaria tigrina (Haw.) Schwantes & Barcelona & 1991 & no & no & 3 synonyms \\
\hline Glottiphyllum cruciatum (Haw.) N.E.Br. & Padova & 2014 & no & no & $10+$ synonyms \\
\hline Glottiphyllum longum (Haw.) N.E.Br. & Pecs & 2001 & no & no & one of $15+$ synonyms \\
\hline Glottiphyllum oligocarpum L. Bolus & Bochum & 1995 & no & no & \\
\hline Glottiphyllum regium N.E.Br. & Pecs & 2017 & no & no & $\begin{array}{l}\text { arrived as syn. G. } \\
\text { compressum }\end{array}$ \\
\hline Glottiphyllum salmii (Haw.) N.E.Br. & Nantes & 1995 & no & no & $\begin{array}{l}\text { syn. Mesembryanthemum } \\
\text { salmii }\end{array}$ \\
\hline Hereroa calycina L. Bolus & Pecs & 2017 & no & no & \\
\hline Hereroa gracilis L. Bolus var. gracilis L. Bolus & Wuppertal & 1998 & no & no & arrived as $H$. gracilis \\
\hline Hereroa muiri L. Bolus & Budapest & 2017 & no & no & \\
\hline Lampranthus aurantiacus Schwantes & Monaco & 1991 & no & no & \\
\hline Lampranthus lunatus N.E. Br. & Berlin- Dahlem & 1979 & no & no & \\
\hline
\end{tabular}




\begin{tabular}{|c|c|c|c|c|c|}
\hline Scientific name & $\begin{array}{l}\text { City of plant } \\
\text { material origin }\end{array}$ & $\begin{array}{c}\text { Year of } \\
\text { ordering/ } \\
\text { purchasing }\end{array}$ & CITES & EASIN & Notes and remarks \\
\hline Lithops marmorata (N.E.Br.) N.E.Br. & Innsbruck & $\begin{array}{l}2008 \text { (seeds } \\
\text { from 2002) }\end{array}$ & no & no & 5 synonyms \\
\hline \multirow[t]{2}{*}{ Lithops salicola L. Bolus } & Dresden & 1986 & no & no & \\
\hline & Cluj-Napoca & $\begin{array}{l}2017 \text { (seeds } \\
\text { from 2015) \& } \\
2017\end{array}$ & no & no & \\
\hline Machairophyllum albidum (L.) Schwantes & Vienna & $\begin{array}{l}2010 \text { (seed } \\
\text { from 1971) }\end{array}$ & no & no & 3 synonyms \\
\hline $\begin{array}{l}\text { Mesembryanthemum splendens L. } \\
\text { subsp. pentagonum (L. Bolus) Klak }\end{array}$ & nn & $\begin{array}{l}\text { in collection } \\
\text { in } 1963\end{array}$ & no & no & $\begin{array}{l}\text { arrived as Aridaria } \\
\text { pentagona; one of } 15+ \\
\text { synonyms }\end{array}$ \\
\hline Mestoklema tuberosum (L.) N.E.Br. & Tuebingen & 2016 & no & no & 4 synonyms \\
\hline Nanathus aloides (Haw.) Schwantes & Tuebingen & $\begin{array}{l}2015 \text { (seeds } \\
\text { from 2013) }\end{array}$ & no & no & \\
\hline \multirow[t]{2}{*}{ Oscularia deltoides (L.) Schwantes } & Siena & $2012 \& 2016$ & no & no & 2 synonyms \\
\hline & Padova & $\begin{array}{l}2017 \text { (seeds } \\
\text { from 2010) }\end{array}$ & no & no & \\
\hline \multirow[t]{2}{*}{ Pleiospilos bolusii (Hook. f.) N.E. Br. } & Kiel & 1995 & no & no & $\begin{array}{l}\text { syn. Mesembryanthemum } \\
\text { bolusii }\end{array}$ \\
\hline & Innsbruck & 2000 & no & no & \\
\hline $\begin{array}{l}\text { Pleiospilos compactus Schwantes subsp. canus } \\
\text { H.E.K. Hartmann \& Liede }\end{array}$ & Innsbruck & 2000 & no & no & $\begin{array}{l}\text { arrived as } P . \text { nobilis; one of } \\
15+\text { synonyms }\end{array}$ \\
\hline Pleiospilos simulans N.E. Br. & Innsbruck & 2000 & no & no & \\
\hline Rhombophyllum dolabriforme Schwantes & Pecs & 2001 & no & no & \\
\hline Schwantesia pillansii L. Bolus & Innsbruck & unknown & no & no & \\
\hline \multicolumn{6}{|l|}{ Apocynaceae } \\
\hline \multirow[t]{2}{*}{ Adenium obesum (Forssk.) Roem. \& Schult. } & Košice & 2016 & no & no & $10+$ synonyms \\
\hline & Prague & 2016 & no & no & \\
\hline $\begin{array}{l}\text { Adenium obesum (Forssk.) Roemer \& Schultes } \\
\text { ssp. obesum }\end{array}$ & Frankfurt & 2017 & no & no & $\begin{array}{l}\text { unknown in The Plant List } \\
\text { and Tropicos }\end{array}$ \\
\hline Plumeria obtusa L. cult. & $\mathrm{nn}$ & 2003 & no & no & unknown cultivar \\
\hline Plumeria rubra L. & $\mathrm{nn}$ & 2002 & no & yes & $\begin{array}{l}\text { gift from Mrs. Ivanka } \\
\text { Gajšak; P. V. }\end{array}$ \\
\hline \multicolumn{6}{|l|}{ Asclepiadaceae } \\
\hline Caralluma europaea (Guss.) N.E.Br. & Duisburg & 1989 & no & no & $30+$ synonyms \\
\hline Caralluma joannis Maire & Monaco & 1997 & no & no & syn. Apteranthes joannis \\
\hline Ceropegia affinis Vatke & $\mathrm{nn}$ & 1969 & no & no & $15+$ synonyms \\
\hline $\begin{array}{l}\text { Ceropegia africana R.Br. subsp. barklyi (Hook.f.) } \\
\text { Bruyns }\end{array}$ & nn & $\begin{array}{l}\text { in collection } \\
\text { in } 1963\end{array}$ & no & no & $\begin{array}{l}\text { arrived as C. barklyi; one of } 2 \\
\text { synonyms }\end{array}$ \\
\hline Ceropegia bulbosa Roxb. & Kaunas & 1970 & no & no & 9 synonyms \\
\hline Ceropegia linearis E.Mey. & Cluj-Napoca & 2003 & no & no & P.V. \\
\hline $\begin{array}{l}\text { Ceropegia linearis E.Mey. subsp. debilis } \\
\text { (N.E.Br.) H.Huber }\end{array}$ & nn & $\begin{array}{l}\text { in collection } \\
\text { in } 1963\end{array}$ & no & no & arrived as syn. C. debilis \\
\hline Ceropegia sandersonii Decne. ex Hook. f. & $\mathrm{nn}$ & 2000 & no & no & syn. C. monterioae \\
\hline Ceropegia stapeliiformis Haw. & Muenchen & 2015 & no & no & $\begin{array}{l}\text { syn. C. stapeliiformis subsp. } \\
\text { stapeliiformis }\end{array}$ \\
\hline Huernia barbata (Masson) Haw. & Basel & 1995 & no & no & 2 synonyms \\
\hline Huernia hystrix (Hook. f.) N.E. Br. & Innsbruck & 1996 & no & no & 3 synonyms \\
\hline \multirow[t]{2}{*}{$\begin{array}{l}\text { Huernia macrocarpa (A. Rich.) Spreng. } \\
\text { f. macrocarpa }\end{array}$} & Basel & 1995 & no & no & $\begin{array}{l}\text { arrived as H. macrocarpa var. } \\
\text { penzigii; one of } 10 \text { synonyms }\end{array}$ \\
\hline & Muenchen & 1995 & no & no & \\
\hline \multirow[t]{2}{*}{ Huernia pendula E. A. Bruce } & Ferrara & 1983 & no & no & \\
\hline & Szeged & 1985 & no & no & \\
\hline $\begin{array}{l}\text { Orbea lutea (N.E. Br.) Bruyns subsp. vaga } \\
\text { (N.E.Br.) Bruyns }\end{array}$ & Pecs & 1996 & no & no & 4 synonyms \\
\hline Orbea variegata (L.) Haw. & Duisburg & 1989 & no & no & $\begin{array}{l}\text { arrived as Stapelia lepida; one } \\
\text { of } 20+\text { synonyms }\end{array}$ \\
\hline
\end{tabular}


S72 Sandev, D. et al.: Plethora of plants - collections of the Botanical Garden, Faculty of Science, University of Zagreb (2)

\begin{tabular}{|c|c|c|c|c|c|}
\hline Scientific name & $\begin{array}{l}\text { City of plant } \\
\text { material origin }\end{array}$ & $\begin{array}{l}\text { Year of } \\
\text { ordering/ } \\
\text { purchasing }\end{array}$ & CITES & EASIN & Notes and remarks \\
\hline Stapelia gigantea N.E. Br. & Besancon & 1970 & no & no & 5 synonyms \\
\hline \multirow[t]{2}{*}{ Stapelia grandiflora Masson } & Gent & 1979 & no & no & one of 20 synonyms \\
\hline & Klagenfurt & 2011 & no & no & \\
\hline \multicolumn{6}{|l|}{ Asteraceae } \\
\hline Senecio rowleyanus $\mathrm{H}$. Jacobsen & Salaspils & 1997 & no & no & P.V. \\
\hline Senecio serpens G. D. Rowley & Genova & 1970 & no & no & 2 synonyms \\
\hline Senecio spiculosus (Shepherd) Rowley & Gent & 1960 & no & no & \\
\hline Senecio tropaeolifolius MacOwan ex F.Muell. & Antwerpen & 1969 & no & no & \\
\hline \multicolumn{6}{|l|}{ Cactaceae } \\
\hline \multirow[t]{2}{*}{$\begin{array}{l}\text { Astrophytum capricorne (A.Dietr.) Britton \& } \\
\text { Rose }\end{array}$} & Cluj-Napoca & 2017 & yes & no & $\begin{array}{l}\text { arrived as A. senile; one of } \\
20+\text { synonyms }\end{array}$ \\
\hline & Monaco & 1991 & yes & no & \\
\hline \multirow[t]{2}{*}{ Astrophytum myriostigma Lem. } & Duesseldorf & 1994 & yes & no & $20+$ synonyms \\
\hline & Parma & 2016 & yes & no & \\
\hline \multirow[t]{4}{*}{ Astrophytum ornatum (DC.) Britton \& Rose } & Frankfurt & 1992 & yes & no & $15+$ synonyms \\
\hline & Košice & $\begin{array}{l}2015 \text { (seeds } \\
\text { from 2013) } \\
\quad \& 2015\end{array}$ & yes & no & \\
\hline & Cluj-Napoca & 2014 & yes & no & \\
\hline & Jibou & 2015 & yes & no & \\
\hline \multirow[t]{2}{*}{$\begin{array}{l}\text { Austrocylindropuntia vestita (Salm-Dyck) } \\
\text { Backeb. }\end{array}$} & Kiev & 1989 & yes & no & $5+$ synonyms \\
\hline & Parma & 2016 & yes & no & \\
\hline Aylostera deminuta (F.A.C.Weber) Backeb. & Innsbruck & 2001 & yes & $?$ & $\begin{array}{l}\text { unresolved name (The Plant } \\
\text { List); } 2 \text { synonyms }\end{array}$ \\
\hline Browningia hertlingiana (Backeb.) Buxb. & Belveder & 1971 & yes & no & $\begin{array}{l}\text { arrived as Azureocereus } \\
\text { hertlingianus; one of } 3 \\
\text { synonyms; natural locality }\end{array}$ \\
\hline Cereus alacriportanus Pfeiff. & Vienna & 1968 & yes & $?$ & $\begin{array}{l}\text { unresolved name (The Plant } \\
\text { List) }\end{array}$ \\
\hline Cereus hexagonus (L.) Mill. & Adelaide & 1972 & yes & no & $15+$ synonyms \\
\hline Cereus jamacaru DC. & Blanes & 1994 & yes & no & 8 synonyms \\
\hline \multirow[t]{3}{*}{ Cereus repandus (L.) Mill. } & nn & 1963 & yes & no & $\begin{array}{l}\text { arrived as C. peruvianus; one } \\
\text { of } 20+\text { synonyms }\end{array}$ \\
\hline & Siena & 2013 & yes & no & \\
\hline & Košice & 2017 & yes & no & \\
\hline Cereus repandus (L.) Mill. f. monstrosa DC. & nn & 2017 & yes & no & $\begin{array}{l}\text { arrived as C. peruvianus } \mathrm{f} . \\
\text { monstrosa }\end{array}$ \\
\hline \multirow[t]{5}{*}{ Cleistocactus straussii (Heese) Backeb. } & Adelaide & 1959 & yes & no & 5 synonyms \\
\hline & Halle & 1966 & yes & no & \\
\hline & Barcelona & 1995 & yes & no & natural locality \\
\hline & Dresden & 1995 & yes & no & \\
\hline & Porrentruy & 1995 & yes & no & \\
\hline $\begin{array}{l}\text { Copiapoa echinoides (Lem. ex Salm-Dyck) } \\
\text { Britton \& Rose }\end{array}$ & nn & 1963 & yes & no & $\begin{array}{l}\text { arrived as Echinocactus } \\
\text { salm-dyckianus; one of } 8 \\
\text { synonyms }\end{array}$ \\
\hline $\begin{array}{l}\text { Cumulopuntia sphaerica (Foerster) E.F. } \\
\text { Anderson }\end{array}$ & nn & 1962 & yes & no & $\begin{array}{l}\text { arrived as Tephrocactus } \\
\text { sphaerica; one of } 10+ \\
\text { synonyms }\end{array}$ \\
\hline $\begin{array}{l}\text { Denmoza rhodacantha (Salm-Dyck) Britton \& } \\
\text { Rose }\end{array}$ & Bordeaux & 1992 & yes & no & $\begin{array}{l}\text { arrived as D. erythrocephala; } \\
\text { one of } 8 \text { synonyms }\end{array}$ \\
\hline \multirow[t]{2}{*}{ Disocactus flagelliformis (L.) Barthlott } & Jibou & 1986 & yes & no & $\begin{array}{l}\text { arrived as Cereus } \\
\text { flagelliformis; one of } 30+ \\
\text { synonyms }\end{array}$ \\
\hline & Jibou & 1986 & yes & no & $\begin{array}{l}\text { arrived as Aporocactus } \\
\text { flagelliformis }\end{array}$ \\
\hline Echinocactus grusonii Hildm. & Basel & $\begin{array}{l}2017 \text { (seeds } \\
\text { from 2014) }\end{array}$ & yes & no & 4 synonyms \\
\hline
\end{tabular}




\begin{tabular}{|c|c|c|c|c|c|}
\hline Scientific name & $\begin{array}{l}\text { City of plant } \\
\text { material origin }\end{array}$ & $\begin{array}{l}\text { Year of } \\
\text { ordering/ } \\
\text { purchasing }\end{array}$ & CITES & EASIN & Notes and remarks \\
\hline & Košice & 2015 & yes & no & \\
\hline & Parma & 2015 & yes & no & \\
\hline & Barcelona & 1980 & yes & no & \\
\hline Echinopsis backebergii Werderm. & Heidelberg & 1967 & yes & no & 6 synonyms \\
\hline Echinopsis calochlora K.Schum. & Meise & 2001 & yes & no & 2 synonyms \\
\hline $\begin{array}{l}\text { Epiphyllum hookeri Haw. subsp. pittieri } \\
\text { (F.A.C.Weber) Ralf Bauer }\end{array}$ & Saarbrucken & 1968 & yes & no & $\begin{array}{l}\text { arrived as E. pittieri; one of } 3 \\
\text { synonyms }\end{array}$ \\
\hline Epiphyllum oxypetalum (DC.) Haw. & Napoli & 1961 & yes & no & $\begin{array}{l}\text { arrived Phyllocactus latifrons; } \\
\text { one od } 10+\text { synonyms }\end{array}$ \\
\hline Epiphyllum phyllanthus (L.) Haw. & Berlin-Dahlem & 1971 & yes & no & $10+$ synonyms \\
\hline Espostoa lanata (Kunth) Britton \& Rose & Barcelona & 1991 & yes & no & $30+$ synonyms \\
\hline \multirow[t]{2}{*}{ Ferocactus glaucescens (DC.) Britton \& Rose } & Palermo & $1957 \& 1962$ & yes & no & $\begin{array}{l}\text { arrived as F. glaucescens var. } \\
\text { pfeifferi }\end{array}$ \\
\hline & Balchik & $\begin{array}{l}2017 \text { (seeds } \\
\text { from 2012) }\end{array}$ & yes & no & \\
\hline Ferocactus wislizeni (Engelm.) Britton \& Rose & Meise & 1994 & yes & no & $15+$ synonyms \\
\hline $\begin{array}{l}\text { Gymnocalycium denudatum (Link \& Otto) } \\
\text { Pfeiff. ex Mittler }\end{array}$ & Dresden & 1997 & yes & no & 9 synonyms \\
\hline Gymnocalycium mostii (Gürke) Britton \& Rose & Monaco & 2004 & yes & no & 2 synonyms \\
\hline Gymnocalycium saglionis (Cels) Britton \& Rose & Linz & 2008 & yes & no & 3 synonyms \\
\hline $\begin{array}{l}\text { Gymnocalycium saglionis (Cels) Britton \& Rose } \\
\text { subsp. tilcarense (Backeb.) H.Till \& W.Till }\end{array}$ & Monaco & 2000 & yes & no & $\begin{array}{l}\text { arrived as G. saglionis var. } \\
\text { tilcarense; } 3 \text { synonyms }\end{array}$ \\
\hline Hylocereus undatus (Haw.) Britton \& Rose & Berlin-Dahlem & 1973 & yes & yes & 7 synonyms \\
\hline Isolatocereus dumortieri (Scheidw.) Backeb. & Beograd & 1964 & yes & no & 4 synonyms \\
\hline Leuchtenbergia principis Hook. & Dresden & 1993 & yes & no & \\
\hline \multirow[t]{2}{*}{ Mammilaria compressa DC. } & Muenchen & 1953 & yes & no & 5+ synonyms \\
\hline & Gent & 1968 & yes & no & arrived as M. seitziana \\
\hline Mammillaria crinita DC. subsp. crinita & Berlin-Dahlem & 1978 & yes & no & $\begin{array}{l}\text { arrived as M. pygmaea; one } \\
\text { of } 3 \text { synonyms }\end{array}$ \\
\hline Mammillaria heyderi Muehlenpf. & Halle & 1965 & yes & no & \\
\hline $\begin{array}{l}\text { Mammillaria karwinskiana Mart. subsp. } \\
\text { nejapensis (R.T. Craig \& E.Y. Dawson) D.R. } \\
\text { Hunt }\end{array}$ & Cluj-Napoca & 2005 & yes & no & $\begin{array}{l}\text { arrived as } M . \text { nejapensis; one } \\
\text { of } 2 \text { synonyms }\end{array}$ \\
\hline Mammillaria mollihamata Shurly & Berlin-Dahlem & 1978 & yes & no & $\begin{array}{l}\text { unresolved name (The Plant } \\
\text { List) }\end{array}$ \\
\hline Mammillaria nana Backeb. & Halle & 1997 & yes & no & \\
\hline Mammillaria perbella Hildm. ex K. Schum. & Dresden & 1997 & yes & no & $\begin{array}{l}\text { arrived as Neomammillaria } \\
\text { perbella }\end{array}$ \\
\hline Mammillaria polyedra Mart. & Dresden & 1996 & yes & no & syn. Mammillaria multiseta \\
\hline Mammillaria prolifera (Mill.) Haw. & Halle & 1984 & yes & no & 2 synonyms \\
\hline $\begin{array}{l}\text { Mammillaria rhodantha Link \& Otto subsp. } \\
\text { mollendorffiana (Shurly) D.R. Hunt }\end{array}$ & Halle & 1998 & yes & no & $\begin{array}{l}\text { arrived as syn. } M \text {. } \\
\text { mollendorfiana }\end{array}$ \\
\hline $\begin{array}{l}\text { Myrtillocactus geometrizans (Mart. ex Pfeiff.) } \\
\text { Console }\end{array}$ & Barcelona & 1971 & yes & no & 3 synonyms \\
\hline Neobuxbaumia euphorbioides Buxb. & Dresden & 2000 & yes & no & $\begin{array}{l}\text { arrived as Rooksbya } \\
\text { euphorbioides; one of 10+ } \\
\text { synonyms }\end{array}$ \\
\hline $\begin{array}{l}\begin{array}{l}\text { Neomammillaria seideliana (Quehl) Britton \& } \\
\text { Rose }\end{array} \\
\end{array}$ & Potsdam & 1994 & no & no & $\begin{array}{l}\text { arrived as Mammillaria } \\
\text { seideliana }\end{array}$ \\
\hline \multirow[t]{3}{*}{ Opuntia ficus-indica (L.) Mill. } & $\mathrm{nn}$ & $\begin{array}{l}\text { in collection } \\
\text { in } 1978\end{array}$ & yes & yes & 4 synonyms \\
\hline & nn & $\begin{array}{l}\text { in collection } \\
\text { in } 2010\end{array}$ & yes & yes & arrived as O. vulgaris \\
\hline & Balchik & $\begin{array}{l}2017 \text { (seeds } \\
\text { from 2012) }\end{array}$ & yes & yes & \\
\hline Opuntia humifusa (Raf.) Raf. & Balchik & $\begin{array}{l}2017 \text { (seeds } \\
\text { from 2012) }\end{array}$ & yes & no & $\begin{array}{l}\text { arrived as O. compressa; one } \\
\text { of } 15+\text { synonyms }\end{array}$ \\
\hline
\end{tabular}


S74 Sandev, D. et al.: Plethora of plants - collections of the Botanical Garden, Faculty of Science, University of Zagreb (2)

\begin{tabular}{|c|c|c|c|c|c|}
\hline Scientific name & $\begin{array}{l}\text { City of plant } \\
\text { material origin }\end{array}$ & $\begin{array}{l}\text { Year of } \\
\text { ordering/ } \\
\text { purchasing }\end{array}$ & CITES & EASIN & Notes and remarks \\
\hline & Parma & 2016 & yes & no & \\
\hline & Balchik & $\begin{array}{l}2017 \text { (seeds } \\
\text { from 2012) }\end{array}$ & yes & no & \\
\hline & Lisbon & unknown & yes & no & \\
\hline Opuntia maxima Mill. & Coimbra & 1996 & yes & yes & $10+$ synonyms \\
\hline \multirow[t]{2}{*}{ Opuntia microdasys (Lehm.) Pfeiff. } & Barcelona & 1994 & yes & yes & 7 synonyms \\
\hline & nn & 1961 & yes & yes & $\begin{array}{l}\text { arrived as O. micodasys var. } \\
\text { albispina }\end{array}$ \\
\hline $\begin{array}{l}\text { Parodia microsperma (F.A.C.Weber) Speg. } \\
\text { subsp. horrida (F.W. Brandt) R. Kiesling \& O. } \\
\text { Ferrari }\end{array}$ & Cluj-Napoca & 2000 & yes & no & $\begin{array}{l}\text { arrived as } P . \text { formosa; one of } \\
10+\text { synonyms }\end{array}$ \\
\hline $\begin{array}{l}\text { Parodia mueller-melchersii (Frič ex Backeb.) } \\
\text { N.P. Taylor }\end{array}$ & Cluj-Napoca & 2017 & yes & no & $\begin{array}{l}\text { arrived as Notocactus } \\
\text { rutilans; one of } 6 \text { synonyms }\end{array}$ \\
\hline Parodia mutabilis Backeb. & Cluj-Napoca & 2005 & yes & $?$ & $\begin{array}{l}\text { unresolved name (The Plant } \\
\text { List); } 2 \text { synonyms }\end{array}$ \\
\hline Pereskia aculeata Mill. & nn & $\begin{array}{l}\text { in collection } \\
\text { in } 1961\end{array}$ & yes & no & $15+$ synonyms \\
\hline Pereskia bleo (Kunth) DC. & Hamma & 1960 & yes & no & 6 synonyms \\
\hline Pereskia sacharosa Griseb. & Košice & 2017 & yes & no & 5 synonyms \\
\hline Pilosocereus coerulescens (Lem.) F.Ritter & Barcelona & 1994 & yes & $?$ & $\begin{array}{l}\text { arrived as Cereus } \\
\text { coerulescens; } 6 \text { synonyms; } \\
\text { unresolved name (The Plant } \\
\text { List) }\end{array}$ \\
\hline $\begin{array}{l}\text { Rebutia deminuta (F.A.C. Weber) Britton \& } \\
\text { Rose }\end{array}$ & Jibou & 1998 & yes & & $\begin{array}{l}\text { arrived as Aylostera } \\
\text { deminuta; one of } 3 \text { synonyms }\end{array}$ \\
\hline Rebutia fiebrigii (Gürke) Britton \& Rose & Cluj-Napoca & 2000 & yes & no & $\begin{array}{l}\text { arrived as Aylostera jujuyana; } \\
\text { one of } 40+\text { synonyms }\end{array}$ \\
\hline Rebutia minuscula K. Schum. & Cluj-Napoca & 2000 & yes & no & $20+$ synonyms \\
\hline Selenicereus grandiflorus (L.) Britton \& Rose & Barcelona & 1971 & yes & no & $20+$ synonyms \\
\hline $\begin{array}{l}\text { Selenicereus macdonaldiae (Hook.) Britton \& } \\
\text { Rose }\end{array}$ & Siena & 1968 & yes & no & 5 synonyms \\
\hline Selenicereus nelsonii (Weing.) Britton \& Rose & Berlin-Dahlem & 1966 & yes & no & syn. Cereus nelsonii \\
\hline $\begin{array}{l}\text { Selenicereus vagans (K. Brandegee) Britton \& } \\
\text { Rose }\end{array}$ & nn & $\begin{array}{l}\text { in collection } \\
\text { in } 1971\end{array}$ & yes & no & 5 synonyms \\
\hline Stetsonia coryne (Salm-Dyck) Britton \& Rose & Berlin- Dahlem & 1987 & yes & no & syn. Cereus coryne \\
\hline Sulcorebutia steinbachii (Werderm.) Backeb. & Bratislava & 2000 & yes & no & $\begin{array}{l}\text { arrived as S. glomerispina; } \\
\text { one of } 20+\text { synonyms }\end{array}$ \\
\hline \multicolumn{6}{|l|}{ Campanulaceae } \\
\hline \multirow[t]{2}{*}{ Canarina canariensis (L.) Vatke } & Wuppertal & 1984 & no & no & 5 synonyms \\
\hline & Dresden & 2006 & no & no & 6 synonyms \\
\hline \multicolumn{6}{|l|}{ Crassulaceae } \\
\hline Adromischus cooperi (Baker) A. Berger & Berlin-Dahlem & 1995 & no & no & 7 synonyms \\
\hline Aeonium arboreum Webb \& Berthel. & Amsterdam & 1976 & no & yes & 9 synonyms \\
\hline $\begin{array}{l}\text { Aeonium arboreum Webb \& Berthel. var. } \\
\text { holochrysum H.Y.Liu }\end{array}$ & Marnier & 1977 & no & yes & 4 synonyms \\
\hline \multirow[t]{2}{*}{ Aeonium canariense (L.) Webb \& Berthel. } & Tuebingen & 2015 & no & no & syn. Sempervivum canariense \\
\hline & Marburg & 2017 & no & no & \\
\hline Aeonium castello-paivae Bolle & Berlin-Dahlem & 1973 & no & no & syn. A. gomeraeum \\
\hline Aeonium decorum Webb ex Bolle & Bristol & 1977 & no & yes & \\
\hline Aeonium dodrantale (Willd.) T. Mes & Marburg & 2017 & no & no & $\begin{array}{l}\text { arrived as Greenovia } \\
\text { dodrantalis; one of } 4 \\
\text { synonyms }\end{array}$ \\
\hline Aeonium glutinosum (Aiton) Webb \& Berthel. & nn & 1988 & no & yes & \\
\hline Aeonium gomerense (Praeger) Praeger & Berlin-Dahlem & 1976 & no & no & syn. Sempervivum gomerense \\
\hline Aeonium goochiae Webb \& Berthel. & nn & 1989 & no & no & 2 synonyms \\
\hline \multirow[t]{2}{*}{ Aeonium haworthii Webb \& Berthel. } & Berlin-Dahlem & 1976 & no & yes & syn. Sempervivum haworthii \\
\hline & Basel & 1995 & no & yes & \\
\hline
\end{tabular}




\begin{tabular}{|c|c|c|c|c|c|}
\hline Scientific name & $\begin{array}{l}\text { City of plant } \\
\text { material origin }\end{array}$ & $\begin{array}{l}\text { Year of } \\
\text { ordering/ } \\
\text { purchasing }\end{array}$ & CITES & EASIN & Notes and remarks \\
\hline $\begin{array}{l}\text { Aeonium percarneum (R. P. Murray) Pitard \& } \\
\text { Proust }\end{array}$ & Gran Canaria & 1994 & no & no & 4 synonyms \\
\hline Aeonium simsii (Sweet) Stearn & Basel & 1989 & no & yes & syn. Sempervivum simsii \\
\hline Aeonium tabuliforme (Haw.) Webb \& Berthel. & Marburg & 2017 & no & no & 5 synonyms \\
\hline Aeonium undulatum Webb \& Berthel. & Tenerife & 1986 & no & no & 2 synonyms \\
\hline Aichryson bethencourtianum Bolle & Szeged & 1996 & no & no & $\begin{array}{l}\text { arrived as Aeonium } \\
\text { bethencourtianum; one of } 4 \\
\text { synonyms }\end{array}$ \\
\hline $\begin{array}{l}\text { Bryophyllum fedtschenkoi (Raym.-Hamet \& } \\
\text { H.Perrier) Lauz.-March. }\end{array}$ & Ferrara & 1970 & no & yes & $\begin{array}{l}\text { arrived as Kalanchoe } \\
\text { fedtschenkoi; one of } 2 \\
\text { synonyms }\end{array}$ \\
\hline $\begin{array}{l}\text { Crassula exilis Harv. subsp. schmidtii (Regel) } \\
\text { G. D. Rowley }\end{array}$ & MBM - bought & 2012 & no & no & $\begin{array}{l}\text { arrived as } C \text {. schmidtii; one } \\
\text { of } 2 \text { synonyms; P.V. }\end{array}$ \\
\hline $\begin{array}{l}\text { Crassula montana L.f. subsp. quadrangularis } \\
\text { (Schönl.) Toelken }\end{array}$ & Wageningen & 1967 & no & no & $\begin{array}{l}\text { arrived as } C \text {. gillii; one of } 2 \\
\text { synonyms; from cuttings }\end{array}$ \\
\hline \multirow[t]{2}{*}{ Crassula muscosa L. var. muscosa } & Wageningen & 1977 & no & no & $\begin{array}{l}\text { arrived as C. lycopodioides } \\
\text { var. purpusii }\end{array}$ \\
\hline & Cluj-Napoca & 2006 & no & no & \\
\hline Crassula ovata (Mill.) Druce & Wageningen & 1964 & no & yes & 8 synonyms \\
\hline \multirow[t]{2}{*}{ Crassula perforata Thunb. } & Besancon & 1971 & no & no & $\begin{array}{l}\text { arrived as C. conjuncta, } 10 \\
\text { synonyms }\end{array}$ \\
\hline & Dresden & 2002 & no & no & arrived as C. perfossa Lam. \\
\hline Crassula socialis Schönland & Caen & 1996 & no & no & \\
\hline Dudleya caespitosa (Haw.) Britton \& Rose & Berlin- Dahlem & 1970 & no & no & $10+$ synonyms \\
\hline Echeveria amoena De Smet ex E.Morren & Szeged & 1999 & no & no & syn. E. pusilla \\
\hline Echeveria derenbergii J. A. Purpus & Antwerpen & 1967 & no & no & \\
\hline Echeveria elegans Rose & $\mathrm{nn}$ & $\begin{array}{l}\text { in collection } \\
\text { in } 1968\end{array}$ & no & no & 4 synonyms \\
\hline Echeveria $x$ gilva E. Walther & Wageningen & 1968 & no & no & E. agavoides $\times$ E. elegans \\
\hline Echeveria nodulosa (Baker) Otto & nn & $\begin{array}{l}\text { in collection } \\
\text { in } 1961\end{array}$ & no & no & $\begin{array}{l}\text { arrived as syn. Cotyledon } \\
\text { nodulosa }\end{array}$ \\
\hline Echeveria pringlei (S.Watson) Rose & $\mathrm{nn}$ & $\begin{array}{c}\text { in collection } \\
\text { in } 1961\end{array}$ & no & no & syn. Cotyledon pringlei \\
\hline Echeveria $\mathrm{x}$ pulchella A. Berger pro sp. & nn & unknown & no & no & $\begin{array}{l}\text { from cuttings; garden } \\
\text { hybrid }\end{array}$ \\
\hline Echeveria pulvinata Rose & nn & $\begin{array}{c}\text { in collection } \\
\text { in } 1961\end{array}$ & no & no & syn. Cotyledon pulvinata \\
\hline Graptopetalum macdougallii Alexander & Wageningen & 1970 & no & no & \\
\hline $\begin{array}{l}\text { Graptopetalum paraguayense (N.E.Br.) } \\
\text { E.Walther }\end{array}$ & Wageningen & 1957 & no & yes & $5+$ synonyms \\
\hline Kalanchoe beharensis Drake & Siena & 2016 & no & no & \\
\hline $\begin{array}{l}\text { Kalanchoe daigremontiana Raym.-Hamet \& H. } \\
\text { Perrier }\end{array}$ & $\mathrm{nn}$ & 1983 & no & yes & $\begin{array}{l}\text { syn. Bryophyllum } \\
\text { daigremontianum; from } \\
\text { cuttings; gift from Eric }\end{array}$ \\
\hline Kalanchoe hildebrandtii Baill. & Wageningen & 1964 & no & no & 3 synonyms \\
\hline Kalanchoe tashiroi Yamam. & nn & 1995 & no & no & \\
\hline Pachyphytum compactum Rose 'Cristatum' & Wageningen & 1965 & no & no & $\begin{array}{l}\text { unknown cultivar, probably } \\
\text { P. compactum f. cristatus } \\
\text { hort. }\end{array}$ \\
\hline Sedum adolphii Raym.-Hamet & nn & $\begin{array}{l}\text { in collection } \\
\text { in } 1963\end{array}$ & no & no & \\
\hline Sedum allantoides Rose & nn & $\begin{array}{l}\text { in collection } \\
\text { in } 1961\end{array}$ & no & no & \\
\hline Sedum compressum Rose & nn & $\begin{array}{l}\text { in collection } \\
\text { in } 1963\end{array}$ & no & no & \\
\hline Sedum goldmanii (Rose) Moran & Pecs & 1997 & no & no & $\begin{array}{l}\text { arrived as Villadia batesii; } \\
\text { one of } 2 \text { synonyms, P.V. }\end{array}$ \\
\hline Sedum lucidum R.T. Clausen & Wageningen & 1967 & no & no & from cuttings \\
\hline
\end{tabular}


S76 Sandev, D. et al.: Plethora of plants - collections of the Botanical Garden, Faculty of Science, University of Zagreb (2)

\begin{tabular}{|c|c|c|c|c|c|}
\hline Scientific name & $\begin{array}{l}\text { City of plant } \\
\text { material origin }\end{array}$ & $\begin{array}{c}\text { Year of } \\
\text { ordering/ } \\
\text { purchasing }\end{array}$ & CITES & EASIN & Notes and remarks \\
\hline Sedum moranense Kunth & Wageningen & 1980 & no & no & 3 synonyms \\
\hline $\begin{array}{l}\text { Sedum moranense Kunth subsp. grandiflorum } \\
\text { R.T. Clausen }\end{array}$ & Wageningen & 1980 & no & no & $\begin{array}{l}\text { arrived as S. moranense } \\
\text { 'Grandiflorum' }\end{array}$ \\
\hline Sedum morganianum $\mathrm{E}$. Walther & nn & $\begin{array}{l}\text { in collection } \\
\text { in } 2000\end{array}$ & no & yes & \\
\hline Sedum nussbaumerianum Bitter & Rotterdam & 1969 & no & no & \\
\hline Sedum rubrotinctum R. T. Clausen 'Aurora' & Wageningen & 1964 & no & yes & \\
\hline Sedum stahlii Solms & Wageningen & 1970 & no & no & \\
\hline Sedum treleasei Rose & Wageningen & 1964 & no & no & \\
\hline Sempervivum tectorum $\mathrm{L}$. & Meyrin & 1980 & no & no & \\
\hline Sempervivum vicentei $\mathrm{Pau}$ & Dresden & 1995 & no & no & 4 synonyms \\
\hline $\begin{array}{l}\text { Sempervivum wulfenii Hoppe ex Mert. \& } \\
\text { W.D.J.Koch }\end{array}$ & Bonn & 1996 & no & no & syn. Sempervivum globiferum \\
\hline Sinocrassula yunnanensis (Franch.) A. Berger & Tuebingen & 2017 & no & no & 2 synonyms \\
\hline \multicolumn{6}{|l|}{ Euphorbiaceae } \\
\hline Euphorbia alcicornis Baker & Iasi & 1995 & yes & no & \\
\hline Euphorbia balsamifera Aiton & Lisbon & 2000 & yes & no & $\begin{array}{l}\text { arrived as E. balsamifera } \\
\text { subsp. adenensis }\end{array}$ \\
\hline Euphorbia bubalina Boiss. & Cluj-Napoca & $\begin{array}{l}2017 \text { (seeds } \\
\text { from 2014) }\end{array}$ & yes & no & syn. E. laxiflora \\
\hline Euphorbia canariensis L. & nn & 1972 & yes & no & 5 synonyms \\
\hline Euphorbia candelabrum Trémaux ex Kotschy & Monaco & 1994 & yes & yes & 5 synonyms \\
\hline Euphorbia fimbriata Scop. & Szeged & 1971 & yes & no & 4 synonyms \\
\hline Euphorbia globosa (Haw.) Sims & Jibou & 1996 & yes & no & 2 synonyms \\
\hline Euphorbia grandidens Haw. & nn & 1971 & yes & no & 2 synonyms \\
\hline Euphorbia lactea Haw. & $\mathrm{nn}$ & unknown & yes & no & \\
\hline Euphorbia ledienii A. Berger & nn & $\begin{array}{l}\text { in collection } \\
\text { in } 1963\end{array}$ & yes & no & syn. E. ledienii var. ledienii \\
\hline Euphorbia milii Des Moul. & nn & $\begin{array}{l}\text { in collection } \\
\text { in } 1963\end{array}$ & yes & yes & $\begin{array}{l}\text { arrived as E. splendens var. } \\
\text { bojeri; } 10+\text { synonyms }\end{array}$ \\
\hline Euphorbia monteiroi Hook. & Richmond & 2000 & yes & no & 3 synonyms \\
\hline \multirow[t]{2}{*}{ Euphorbia obesa Hook.f. } & Kiel & 1996 & yes & no & syn. E. obesa subsp. obesa \\
\hline & Monaco & 2014 & yes & no & \\
\hline Euphorbia ornithopus Jacq. & Jibou & 1996 & yes & no & \\
\hline Euphorbia pendula Boiss. & nn & 1971 & no & no & \\
\hline Euphorbia pseudocactus A. Berger & Besancon & 1971 & yes & no & \\
\hline Euphorbia tirucalli L. & nn & $\begin{array}{l}\text { in collection } \\
\text { in } 1974\end{array}$ & yes & no & $15+$ synonyms \\
\hline Euphorbia tithymaloides L. & Strasbourg & 1969 & no & no & $\begin{array}{l}\text { arrived as Pedilanthus } \\
\text { tithymaloides; one of } 20+ \\
\text { synonyms }\end{array}$ \\
\hline $\begin{array}{l}\text { Euphorbia tithymaloides L. subsp. smallii } \\
\text { (Millsp.) V.W.Steinm. }\end{array}$ & Rotterdam & 1969 & no & no & $\begin{array}{l}\text { arrived as Pedilanthus smallii; } \\
\text { one of } 3 \text { saynonyms }\end{array}$ \\
\hline Euphorbia umbellata (Pax) Bruyns & nn & $\begin{array}{l}\text { in collection } \\
\text { in } 1968\end{array}$ & yes & yes & $\begin{array}{l}\text { arrived as Synadenium } \\
\text { grantii; one of } 4 \text { synonyms }\end{array}$ \\
\hline Euphorbia umbellata (Pax) Bruyns 'Purpurea' & Besancon & 1978 & no & yes & $\begin{array}{l}\text { arrived as Synadenium } \\
\text { grantii 'Purpurea' }\end{array}$ \\
\hline Euphorbia virosa Willd. & $\mathrm{nn}$ & 1970 & yes & no & 4 synonyms \\
\hline \multicolumn{6}{|l|}{ Geraniaceae } \\
\hline Pelargonium sericifolium J. J. A. van der Walt & Frankfurt & 2017 & no & no & arrived as P. sericeum \\
\hline \multicolumn{6}{|l|}{ Portulacaceae } \\
\hline Anacampseros rufescens (Haw.) Sweet & Goettingen & 1983 & yes & no & $\begin{array}{l}\text { syn. A. arachnoides var. } \\
\text { grandiflora }\end{array}$ \\
\hline \multirow[t]{2}{*}{ Anacampseros telephiastrum DC. } & Frankfurt & 1960 & yes & no & $5+$ synonyms \\
\hline & Jibou & 1998 & yes & no & \\
\hline Portulacaria afra Jacq. & nn & $\begin{array}{l}1998 \text { (seeds } \\
\text { from 1961) }\end{array}$ & no & no & 6 synonyms \\
\hline
\end{tabular}




\begin{tabular}{|c|c|c|c|c|c|}
\hline Scientific name & $\begin{array}{l}\text { City of plant } \\
\text { material origin }\end{array}$ & $\begin{array}{l}\text { Year of } \\
\text { ordering/ } \\
\text { purchasing }\end{array}$ & CITES & EASIN & Notes and remarks \\
\hline \multicolumn{6}{|l|}{ Vitaceae } \\
\hline Cyphostemma juttae (Dinter \& Gilg) Desc. & Monaco & 2012 & no & no & syn. Cissus juttae \\
\hline \multicolumn{6}{|l|}{ MONOCOTYLEDONS } \\
\hline \multicolumn{6}{|l|}{ Agavaceae } \\
\hline Agave americana L. 'Variegata' & $\mathrm{nn}$ & 1972 & no & no & \\
\hline Agave atrovirens Karw. ex Salm-Dyck & $\mathrm{nn}$ & 1968 & no & yes & $15+$ synonyms \\
\hline \multirow[t]{2}{*}{ Agave attenuata Salm-Dyck } & nn & $\begin{array}{l}\text { in collection } \\
\text { in } 1970\end{array}$ & no & yes & $\begin{array}{l}\text { arrived as A. cernum; } 5+ \\
\text { synonyms }\end{array}$ \\
\hline & Genova & 2017 & no & yes & arrived as A. attenuata \\
\hline Agave aurea Brandegee & $\mathrm{nn}$ & $\begin{array}{l}\text { in collection } \\
\text { in } 1981\end{array}$ & no & no & \\
\hline Agave bracteosa S.Watson ex Engelm. & Menton & 2002 & no & no & \\
\hline Agave deserti Engelm. & Jerusalem & 2016 & no & no & $\begin{array}{l}3 \text { synonyms; large and } \\
\text { variable complex }\end{array}$ \\
\hline Agave filifera Salm-Dyck & Giessen & 2015 & no & no & 6 synonyms \\
\hline Agave havardiana Trel. & Wuerzburg & 2017 & no & no & \\
\hline $\begin{array}{l}\text { Agave mitis Mart. var. albidior (Salm-Dyck) } \\
\text { B.Ullrich }\end{array}$ & Nancy & $\begin{array}{c}1962 \& \text { in } \\
\text { collection } \\
\text { in } 2002\end{array}$ & no & no & $\begin{array}{l}\text { arrived as A. albicans; one } \\
\text { of } 10+\text { synonyms }\end{array}$ \\
\hline Agave salmiana Otto ex Salm-Dyck & nn & $\begin{array}{l}\text { in collection } \\
\text { in } 1999\end{array}$ & no & no & $10+$ synonyms \\
\hline $\begin{array}{l}\text { Agave salmiana Otto ex Salm-Dyck var. ferox } \\
\text { (K.Koch) Gentry }\end{array}$ & Barcelona & 1971 & no & yes & $\begin{array}{l}\text { arrived as A. ferox; one of } 3 \\
\text { synonyms }\end{array}$ \\
\hline Agave univittata Haw. & Genova & 2017 & no & no & $\begin{array}{l}\text { arrived as Agave lophantha; } \\
\text { one of } 20+\text { synonyms }\end{array}$ \\
\hline \multirow[t]{4}{*}{ Agave victoriae-reginae T.Moore } & Kiel & 2006 & yes & no & $5+$ synonyms \\
\hline & Monaco & 2011 & yes & no & \\
\hline & Besancon & 2017 & yes & no & \\
\hline & Genova & 2017 & yes & no & \\
\hline Furcraea undulata Jacobi & Berlin- Dahlem & $1989 \& 1996$ & no & no & $\begin{array}{l}\text { arrived as F. pubescens; one } \\
\text { of } 2 \text { synonyms }\end{array}$ \\
\hline Hesperoyucca whipplei (Torr.) Trel. & Berkeley & 1994 & no & no & $\begin{array}{l}\text { arrived as Yucca whipplei; } \\
\text { one of } 20+\text { synonyms }\end{array}$ \\
\hline \multirow[t]{2}{*}{ Manfreda virginica (L.) Salisb. ex Rose } & Frankfurt & 2017 & no & no & $\begin{array}{l}\text { arrived as Agave virginica; } \\
\text { one of } 20+\text { synonyms }\end{array}$ \\
\hline & Weinheim & 2017 & no & no & \\
\hline Yucca aloifolia $\mathrm{L}$. & Barcelona & 1962 & no & yes & $20+$ synonyms \\
\hline Yucca aloifolia L. 'Variegata' & Antibes & 1962 & no & yes & \\
\hline \multicolumn{6}{|l|}{ Aloaceae } \\
\hline Aloe africana Mill. & Kirstenbosch & 1986 & yes & no & 8 synonyms \\
\hline Aloe arborescens Mill. & nn & 1981 & yes & yes & $15+$ synonyms \\
\hline Aloe brevifolia Mill. & Toronto & 1989 & yes & yes & 8 synonyms \\
\hline Aloe capitata Baker & Barcelona & 1986 & yes & no & 2 synonyms \\
\hline Aloe castanea Schönland & Jerusalem & 2016 & yes & no & \\
\hline Aloe ciliaris Haw. & Barcelona & 1962 & yes & yes & 2 synonyms \\
\hline Aloe dichotoma Masson & nn & 1986 & yes & no & 4 synonyms \\
\hline \multirow[t]{3}{*}{ Aloe ferox Mill. } & nn & $\begin{array}{l}\text { in collection } \\
\text { in } 1961\end{array}$ & yes & yes & $\begin{array}{l}\text { from cuttings; } 10+ \\
\text { synonyms }\end{array}$ \\
\hline & Jerusalem & $2012 \& 2014$ & yes & yes & \\
\hline & Basel & 2014 & yes & yes & \\
\hline Aloe grandidentata Salm-Dyck & Kirstenbosch & 1986 & yes & no & \\
\hline Aloe pretoriensis Pole-Evans & Stuttgart & 1997 & yes & no & \\
\hline Aloe ramosissima Pillans & Bonn & 1995 & yes & no & $\begin{array}{l}2+\text { synonyms; from natural } \\
\text { locality }\end{array}$ \\
\hline Aloe reitzii Reynolds & Kiel & 1996 & yes & no & syn. A. reitzii var. reitzii \\
\hline Aloe striata Haw. & Liege & 1986 & yes & no & 5 synonyms \\
\hline
\end{tabular}


S78 Sandev, D. et al.: Plethora of plants - collections of the Botanical Garden, Faculty of Science, University of Zagreb (2)

\begin{tabular}{|c|c|c|c|c|c|}
\hline Scientific name & $\begin{array}{l}\text { City of plant } \\
\text { material origin }\end{array}$ & $\begin{array}{l}\text { Year of } \\
\text { ordering/ } \\
\text { purchasing }\end{array}$ & CITES & EASIN & Notes and remarks \\
\hline Aloe striatula Haw. & Genova & 2016 & yes & no & 5 synonyms \\
\hline Aloe succotrina Lam. & Turku & 2015 & yes & yes & $10+$ synonyms \\
\hline Aloe suprafoliata Pole-Evans & Latte & 1990 & yes & no & \\
\hline \multirow[t]{2}{*}{ Aloe vera (L.) Burm. f. } & Palermo & 1962 & yes & yes & $15+$ synonyms \\
\hline & Siena & $2013 \& 2015$ & yes & no & \\
\hline Aloe zebrina Baker & Monaco & 2017 & yes & no & $10+$ synonyms \\
\hline Gasteria acinacifolia (J.Jacq.) Haw. & Adelaide & 1953 & no & no & $20+$ synonyms \\
\hline Gasteria bicolor Haw. var. bicolor & nn & $\begin{array}{l}\text { in collection } \\
\text { in } 1951\end{array}$ & no & no & $\begin{array}{l}\text { arrived as G. picta var. } \\
\text { formosa; one of } 5 \text { synonyms }\end{array}$ \\
\hline \multirow[t]{2}{*}{$\begin{array}{l}\text { Gasteria bicolor Haw. var. liliputana (Poelln.) } \\
\text { Van Jaarsv. }\end{array}$} & Wageningen & $1964 \& 1971$ & no & no & arrived as G. liliputana \\
\hline & Cluj-Napoca & 1999 & no & no & \\
\hline Gasteria carinata (Mill.) Duval & Kirstenbosch & 1984 & no & no & 4 synonyms \\
\hline \multirow[t]{2}{*}{ Gasteria carinata (Mill.) Duval var. carinata } & Adelaide & 1959 & no & no & $\begin{array}{l}\text { arrived as G. angulata; one of } \\
40+\text { synonyms }\end{array}$ \\
\hline & Berlin-Dahlem & 1978 & no & no & arrived as G. sulcata \\
\hline $\begin{array}{l}\text { Gasteria carinata (Mill.) Duval var. verrucosa } \\
\text { (Mill.) Van Jaarsv. }\end{array}$ & Padova & 2014 & no & no & $10+$ synonyms \\
\hline \multirow[t]{2}{*}{ Haworthia attenuata (Haw.) Haw. } & Wageningen & 1969 & no & no & 2 synonyms \\
\hline & Monaco & 1994 & no & no & \\
\hline $\begin{array}{l}\text { Haworthia attenuata (Haw.) Haw. var. radula } \\
\text { (Jacq.) M.B. Bayer }\end{array}$ & Iasi & 1958 & no & no & 6 synonyms \\
\hline \multirow[t]{2}{*}{ Haworthia coarctata Haw. } & nn & $\begin{array}{l}\text { in collection } \\
\text { in } 1968\end{array}$ & no & no & $20+$ synonyms \\
\hline & Wageningen & 1970 & no & no & \\
\hline Haworthia cymbiformis (Haw.) Duval & Brno & 1963 & no & no & $20+$ synonyms \\
\hline Haworthia fasciata (Willd.) Haw. & Monaco & 1992 & no & no & $15+$ synonyms \\
\hline Haworthia marumiana Uitewall & Wageningen & 1965 & no & no & $\begin{array}{l}\text { syn. H. arachnoidea var. } \\
\text { marumiana }\end{array}$ \\
\hline Haworthia reinwardtii (Salm-Dyck) Haw. & nn & $\begin{array}{l}\text { in collection } \\
\text { in } 1963\end{array}$ & no & no & $10+$ synonyms \\
\hline Hesperaloe parviflora (Torr.) J.M.Coult. & $\begin{array}{l}\text { Davis } \\
\text { (California) }\end{array}$ & 1988 & no & no & 5 synonyms \\
\hline \multicolumn{6}{|l|}{ Bromeliaceae } \\
\hline Dyckia brevifolia Baker & Frankfurt & 1993 & no & no & 4 synonyms \\
\hline \multirow[t]{2}{*}{ Dyckia rariflora Schult. \& Schult.f. } & Hamburg & 1960 & no & no & \\
\hline & Palermo & 1993 & no & no & \\
\hline Hechtia argentea Baker & Barcelona & 1960 & no & no & \\
\hline \multicolumn{6}{|l|}{ Hyacinthaceae } \\
\hline Bowiea volubilis Harv. & Halle & 1961 & no & no & 2 synonyms \\
\hline \multicolumn{6}{|l|}{ Nolinaceae } \\
\hline Calibanus hookeri (Lem.) Trel. & Bochum & 1998 & no & no & 7 synonyms \\
\hline
\end{tabular}

